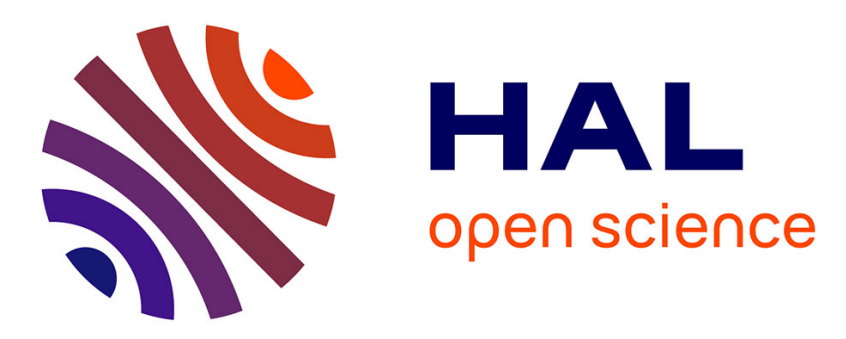

\title{
Le licenciement des salariés protégés : processus et enjeux
}

Mario Correia, Nicole Maggi-Germain

\section{To cite this version:}

Mario Correia, Nicole Maggi-Germain. Le licenciement des salariés protégés : processus et enjeux. 2006. halshs-00361425

\section{HAL Id: halshs-00361425 \\ https://shs.hal.science/halshs-00361425}

Submitted on 14 Feb 2009

HAL is a multi-disciplinary open access archive for the deposit and dissemination of scientific research documents, whether they are published or not. The documents may come from teaching and research institutions in France or abroad, or from public or private research centers.
L'archive ouverte pluridisciplinaire HAL, est destinée au dépôt et à la diffusion de documents scientifiques de niveau recherche, publiés ou non, émanant des établissements d'enseignement et de recherche français ou étrangers, des laboratoires publics ou privés. 


\section{Maison des Sciences de l'Homme Ange Guépin}

Laboratoire droit et changement social

(droit-et-Changement-Social@droit.univ-nantes.fr)

Laboratoire d'Economie et de Sociologie du Travail

(lest@univ-aix.fr)

$* * * * * * *$

\section{LE LICENCIEMENT DES SALARIES PROTEGES. PROCESSUS ET ENJEUX}

\section{RAPPORT DE RECHERCHE}

PROGRAMME DU MINISTERE DE L'EMPLOI, DU TRAVAIL ET DE LA COHESION SOCIALE DARES

(Direction de l'Animation de la Recherche, des Études et des Statistiques)

Sous-direction des salaires, du travail et des relations professionnelles

Département relations professionnelles et temps de travail

Mario CORREIA,

Maître de conférence de sociologie à l'Institut du travail d'Aix en Provence, LEST.

Nicole MAGGI-GERMAIN,

Maître de conférence de droit privé à l'Institut des Sciences Sociales du Travail, Université de Paris I,

Panthéon-Sorbonne, DCS. 


\section{TABLE DES MATIERES}

INTRODUCTION

Présentation de l'appel à projet 5

Le droit et la sociologie pour comprendre le licenciement des salariés protégés 6

La méthodologie juridique retenue $\quad 6$

L'enquête de terrain

La population interrogée $\quad 8$

Le processus conflictuel comme clef de compréhension sociologique 9

$\begin{array}{ll}\text { Plan de lecture } & 10\end{array}$

IERE PARTIE -L'OCCUPATION DU MANDAT PAR LE SALARIE PROTEGE : ANALYSE DES LOGIQUES A L'OEUVRE

I- L'existence d'un cadre juridique contraignant $\quad 13$

A) Le salarié protégé sous mandat $\quad 13$

1) Une protection étendue 13

2) La protection est attachée au mandat et non à la personne 16

B) Les effets de ce mandat $\quad 18$

1) Le mandat confère certaines prérogatives au salarié protégé 19

2) Le mandat confère un statut social au salarié protégé $\quad 21$

3) Le salarié protégé sous contrat et sous statut 23

II- Les différentes configurations du mandat 25

A) La différenciation des positions ou l'opposition des intérêts 26

1) La référence à l'intérêt collectif 26

2) L'idéologisation du mandat ou le primat des principes 30

3) Les effets des candidatures collectives 32

4) Le droit : une ressource garantissant les limites des positions 34

B) La personnalisation des mandats

1) Un positionnement facilité par l'absence de référence aux organisations syndicales 38

2) Des références personnelles à l'intérêt collectif 39

3) Les déterminants des candidatures individuelles $\quad 41$

4) Les effets des candidatures individuelles 48

5) La figure intermédiaire : le passeur à mi-chemin de l'opposition et de l'adhésion 53

III- L'influence des stratégies des entreprises

A) "L'industrialisation" de la gestion du personnel intègre les oppositions 56

B) Le management intégratif ou paternaliste ne supporte pas l'opposition 61

C) Les configurations relationnelles définissent des enjeux différenciés et exigent des ressources particulières 
1) La place croissante occupée par la procédure $\quad 76$

2) Une procédure principalement orale $\quad 78$

B) Une fonction de restitution juridique $\quad 82$

1) La qualification juridique donnée aux faits

2) Une autorité qui dispose de pouvoirs encadrés 85

C) La dimension subjective de la décision de l'inspecteur du travail 87

1) Le reflet d'une certaine représentation, par l'inspecteur du travail, de son rôle 88

2) L'appréhension du contexte 93

3) Les appréciations se fondent sur l'interrelation 100

$\begin{array}{ll}\text { II- Des interventions périphériques annexes } & 101\end{array}$

A) Le comité d'entreprise $\quad 101$

1) Un avis obligatoire 102

2) Un avis qui pèse peu sur l'issue de la procédure 103

B) L'organisation syndicale $\quad 105$

1) La présence syndicale cristallise le conflit 105

2) Le syndicat : une ressource dans le litige 106

Conclusions intermédiaires $\quad 109$

\section{IIIE PARTIE - LE LICENCIEMENT ENTRE ARRANGEMENT ET SORTIE DE CRISE}

I- Le licenciement peut être vécu comme inéluctable ou avantageux 111

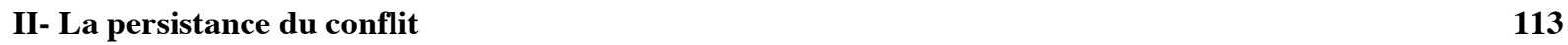

A) Le recours administratif ou contentieux 113

B) La recherche de l'écrasement de l'adversaire $\quad 116$

D) Les ressources sont différentes pour chaque acteur 118

E) Le droit une ressource au service des personnes en cas de conflit $\quad 123$

III- La fin du conflit $\quad 124$

A) L'inaptitude impose la rupture. $\quad 125$

B) L'inaptitude se construit avec le salarié $\quad 126$

1) Un moyen pour le salarié protégé de se retirer d'une situation de travail 126

2) La distorsion de la règle de droit 127

C) Les transactions révélatrices de l'existence d'un départ négocié 130

1) Le cadre social et juridique du recours à la transaction $\quad 130$

2) Le contournement de la règle de droit 133

$\begin{array}{ll}\text { Conclusions intermédiaires } & 139\end{array}$

CONCLUSION GENERALE

$\begin{array}{ll}\text { BIBLIOGRAPHIE } & 147\end{array}$

$\begin{array}{ll}\text { Bibliographie sociologique } & 147\end{array}$

$\begin{array}{lr}\text { Bibliographie juridique } & 148\end{array}$

$\begin{array}{lr}\text { Ouvrages et thèses } & 148\end{array}$

$\begin{array}{lr}\text { Articles } & 149\end{array}$

$\begin{array}{lr}\text { Rapports et études } & 151\end{array}$

$\begin{array}{lr}\text { Documents administratifs } & 152\end{array}$ 


\section{INTRODUCTION}

\section{Présentation de l'appel à projet}

La représentation des salariés dans les entreprises est l'un des principes fondamentaux de notre droit du travail. Les salariés qui l'exercent, investis d'une fonction ou d'un mandat, bénéficient d'un statut protecteur contre le licenciement, afin de leur permettre d'assurer en toute indépendance leur mission. Dans une République qui est, comme le rappelle la constitution, "démocratique et sociale", ce statut protecteur participe de l'expression collective des salariés et de la défense de leurs intérêts.

Telle que mise en œuvre par le Code du travail, la protection des représentants du personnel contre le licenciement possède deux visages :

- une procédure spéciale au terme de laquelle, notamment, un salarié protégé ne peut être licencié que si l'inspecteur du travail a donné une autorisation préalable

- des dispositions plus générales applicables à tous les salariés qui protègent l'exercice du droit syndical (articles L. 411-5, L. 412-6-1 et L. 412-6-2) et interdisent les discriminations (article L. 122-45).

L'habitude a été prise, y compris par les juridictions tant judiciaires qu'administratives, de les regrouper sous une même dénomination: les salariés dits "protégés ». Or, l'expression «salariés protégés » n'a jamais été consacrée par le Code du travail. C'est pourtant cette terminologie qu'utilisent tant le Conseil d'Etat que la Cour de cassation qui se s'y réfèrent expressément dans leurs jugements à partir de 1981 pour le Conseil d'Etat ${ }^{1}$ et à compter de 1978 pour la Chambre sociale de la Cour de cassation ${ }^{23}$.La dénomination, certes commode (les salariés dits "protégés» ne sont pas tous des représentants du personnel), induit cependant certaines représentations. En effet, outre leur statut protecteur, certaines évolutions récentes pourraient inciter à penser que la situation des représentants du personnel dans l'entreprise se renforce: le développement de la négociation collective décentralisée, notamment sur la réduction du temps de travail, les sollicite fortement; le regain de l'action collective, à la faveur de l'amélioration de la situation économique des années 1999 et 2000, comme le montre le net accroissement des journées individuelles non travaillées publiée par la DARES, les rend d'autant plus nécessaires à la négociation pour le règlement des conflits. Or, il s'avère que les salariés dits «protégés » sont aussi des salariés « exposés » ${ }^{4}$.

Dans un premier temps, les demandes de licenciement de salariés protégés ont augmenté mais, semble-t-il, pour des raisons conjoncturelles : sur la première moitié de la décennie 90 , on constate une hausse marquée des demandes de licenciement à partir de 1990 mais qui suit l'évolution de la situation de l'emploi (pic en 1993) et qui ne se confirme pas ensuite.

\footnotetext{
${ }^{1} \mathrm{CE}$, contentieux, 29 avril $1981, \mathrm{n}^{\circ} 22306$, publié au recueil Lebon.

${ }^{2}$ Cass. Soc., 1er février 1978, ${ }^{\circ}$ de pourvoi : 76-40273, publié au bulletin.

${ }^{3}$ Résultats obtenus d'après une recherche effectuée par mots clés sur Légifrance. Si certains arrêts antérieurs contiennent l'expression «salariés protégés », ce n'est qu'en référence aux conclusions des parties.

4 J.-M. Verdier, «Les représentants des salariés : «protégés » ou exposés ? Liberté fondamentale et logique du statut», in Analyse juridique et valeurs en droit social, Mélanges en l'honneur de Jean Pélissier, Dalloz, 2004, 631 p., p. 571 à 590.
} 
Dans un second temps, c'est la stabilité qui prévaut : à partir de 1998, on constate une grande stabilité du rapport salariés protégés licenciés/ensemble des entrées à l'ANPE pour cause de licenciement ; les salariés protégés représentent environ 1,3\% par an des entrées à l'ANPE suite à un licenciement. Globalement, on peut dire que les variations du nombre de demandes de licenciement de salariés protégés suivent la conjoncture plus ou moins bonne de l'emploi.

Ces dernières années (2000-2003) confirment en revanche la part croissante du motif économique dans les demandes de licenciement de salariés protégés. La part des demandes de licenciement pour motif économique visant des salariés protégés s'est accrue alors qu'elle baissé dans l'ensemble des établissements «licencieurs » et parmi les entrées à l'ANPE suite à un licenciement ${ }^{1}$.

Dans le contexte de mise en chantier de la "refondation sociale", qui a amené partenaires sociaux et pouvoirs publics à s'interroger sur les modalités de la représentation des salariés et les conditions de mise en ouvre de la négociation collective, ces résultats appellent des éléments d'interprétation plus précis.

C'est pourquoi, ne disposant actuellement que de données quantitatives issues de remontées administratives, la Dares a fait appel à une équipe de chercheurs pour la réalisation d'une enquête qualitative sur les processus et les enjeux des licenciements de salariés protégés.

\section{Le droit et la sociologie pour comprendre le licenciement des salariés protégés}

\section{La méthodologie juridique retenue}

Le licenciement des salariés protégés ne peut s'analyser et se comprendre sans une incursion dans le droit. En effet, si l'intérêt d'une telle étude ${ }^{2}$ est d'essayer de mieux cerner le processus à l'œuvre, processus qui conduit à la demande de licenciement présentée à l'inspecteur du travail, l'analyse juridique permet de re-situer les pratiques dans un cadre légal contraignant. Ce faisant, il apparaît fondamental de relever et d'analyser les éventuels décalages entre le cadre légal et les pratiques. Quels sont les usages sociaux du droit en la matière, autrement dit, comment les personnes utilisent-elles le droit ? Quel sens donner à d'éventuelles distorsions ou contournements ? Il apparaît donc essentiel de distinguer (ce que nous nous efforçons de faire) l'analyse du cadre juridique, porteur de certaines représentations sociales, de pratiques qui s’en éloignent parfois.

Une telle démarche implique de connaître les textes et d'analyser les pratiques. Le chercheur ne peut en effet comprendre ce qui se joue lors d'un conflit s'il n'a pas la connaissance du cadre juridique qui structure, pour partie, ce conflit. La difficulté réside alors dans l'élaboration d'une "langue commune" au droit et à la sociologie. Certaines notions sociologiques peuvent alors apparaître floues ou, au contraire, trop schématiques pour le juriste qui peut, à l'inverse, sembler quelque peu "s'enfermer" dans une rigueur conceptuelle. Au final, ce travail disciplinaire, bien qu'indispensable, est loin d'être évident.

\footnotetext{
1 Merlier R., De Oliveira V., Zilberman S., "Les licenciements de salariés protégés de 2001 à 2003 : le motif économique prédominant, toujours et encore", Premières informations et premières synthèses n ${ }^{\circ} 38.3,2005$.

${ }^{2} \mathrm{Cf}$. N. Maggi-Germain, «Le licenciement des salaries proteges : processus et enjeux. Synthèse juridique d'une étude menée pour la Dares », à paraître à la revue Droit et société.
} 
Le travail juridique a été réalisé à partir d'une analyse de la jurisprudence, et plus spécifiquement de celle du Conseil d'Etat, réalisée au moyen de deux banques de données : Légisoft, qui contient l'intégralité des arrêts publiés au Recueil Lebon par le Conseil d'Etat depuis 1984 et Légifrance, site Internet offrant l'accès à une banque de données des arrêts du Conseil d'Etat ${ }^{1}$. Un dépouillement et une analyse des arrêts rendus par le Conseil d'Etat a été réalisé sur la période 1990-2002. Une mise à jour a été réalisée, incluant, dans cette étude, les arrêts les plus importants rendus jusqu'en juin 2005.

Le travail d'analyse juridique a été complété par la lecture des différentes circulaires du Ministère des affaires sociales relatives aux salariés protégés ainsi que par la lecture d'articles de doctrine (cf. la bibliographie). Quant à la consultation de "manuels", elle a permis d'apporter des éclaircissements sur des points de droit très précis.

Cette première partie d'un travail basé sur l'analyse de normes juridiques nous a permis d'avoir une vision globale des litiges et des réponses apportées par le droit. L'intérêt est d'ensuite les confronter aux pratiques.

\section{L'enquête de terrain}

Pour cela nous avons fait deux séries d'entretiens :

- Une dizaine d'entretiens avec des salariés protégés licenciés, des inspecteurs du travail du travail, des représentants syndicaux ${ }^{2}$

- À partir du repérage d'une douzaine de dossiers diversifiés une quarantaine d'entretiens ont été réalisés. Dans chaque cas et dans la mesure du possible ont été interrogés : le salarié protégé lui-même, le chef d'entreprise ou son représentant, un représentant du comité d'entreprise, l'inspecteur du travail en charge du dossier.

Nous avons pu ainsi dans une première étape croiser les informations issues de l'enquête et un travail spécifique d'analyse des dossiers ${ }^{3}$. Ainsi ont pu êtres précisés la problématique et le choix de la population à interroger.

Dans la deuxième phase, nous avons alors pu croiser les points de vue différents portés sur une même affaire et effectuer une analyse thématique des entretiens.

En plus de ces entretiens réalisés dans le cadre précis de l'enquête, d'autres entretiens ont eu lieu avec trois médecins du travail (Haute-Normandie et Paris), dont un fait partie de la

\footnotetext{
${ }^{1}$ Publiés au recueil Lebon et aux Tables du recueil Lebon depuis 1965; inédits au recueil Lebon depuis 1986. La publication, décidée par le Conseil d'Etat, rend compte de l'importance de la décision qui présente généralement un intérêt particulier (questions de droit nouvelles ou évolutions jurisprudentielles...). Elles figurent en intégralité dans la première partie de Recueil Labon et sous forme d'analyse dans la seconde partie, appelée aussi Tables du recueil Lebon (il s'agit alors de décisions qui confirment la jurisprudence antérieure ou ne font qu'apporter un complément d'analyse).

${ }^{2}$ Les représentants syndicaux ont une vision assez peu précise de ce qui déroule lors des licenciements de salariés protégés. Il faut certainement mettre ces lacunes en regard du fait que la plus grande partie des demandes de licenciement concerne des non-syndiqués mais aussi que, même chez les syndiqués, le licenciement est géré comme une affaire personnelle et non comme une affaire qui concerne l'organisation syndicale.

${ }^{3}$ L'analyse des dossiers au moyen du logiciel Alceste s'est révélée décevante. Le courrier administratif se prête en effet assez mal à une analyse lexicale.
} 
consultation "souffrance et travail" mise en place par la FNATH (Fédération Nationale des Accidentés du Travail et Handicapés) en mars 2001, dans le cadre d'une expérimentation réalisée avec le soutien du Ministère de l'Emploi et de la Solidarité, du Conseil Régional d'Ile de France et de la Mairie de Paris. Cette consultation vise à répondre à l'insuffisance de prise en charge des situations de détresse psychique de personnes en difficulté dans leur travail, ou à la suite d'accident du travail ou de maladie professionnelle :

- en offrant aux intéressés une écoute psychologique clinique, qui leur permette d'exprimer leur souffrance, de trouver un conseil, éventuellement une orientation thérapeutique spécialisée ;

- en développant la coopération entre les différents acteurs concernés par ces situations complexes où interfèrent des facteurs médicaux, juridiques, sociaux et économiques, et dont l'évolution est directement déterminée par la cohérence des décisions prises.

Le travail réalisé au sein de cette enquête est également le fruit d'un enseignement réalisé à l'ISST, Institut des sciences sociales du travail, UFR de l'Université de Paris 5, PanthéonSorbonne, auprès de militants syndicaux des trois grandes confédérations : CGT, CDFT, FO. Les enseignements se déroulent sur une durée de cinq jours et portent sur un thème précis. Les stages réalisés depuis 2001 avec des conseillers prud'homaux sur les thèmes des "discriminations" ou encore "Santé et travail" ont apporté un éclairage à cette étude.

\section{La population interrogée}

Il est hors de portée d'une équipe comme la nôtre, mais aussi inadéquat avec la problématique choisie, de tenter d'analyser un échantillon représentatif des dossiers de licenciement protégés. Toutefois, le choix ne doit pas non plus reposer sur le hasard. Il nous faut alors viser la significativité de l'échantillon.

Par significatif nous entendons une population présentant la plus grande diversité possible en ce qui concerne les variables descriptives dont dispose chaque DDTE. Nous avons repéré les variables suivantes dont toutes les modalités doivent dans la mesure du possible exister dans la population, de manière à peu près répartie : nature de la demande de licenciement, mandat détenu, syndiqués - non-syndiqués, PCS, âge, sexe...

Nous avons analysé 12 cas de demandes de licenciement répartis sur les deux départements Isère et Hauts-de-Seine. Les motifs des demandes de licenciements sont assez diversifiés : 5 demandes pour motif économique, dont deux procédures gérées par des administrateurs ; 3 demandes pour faute ; 4 demandes pour inaptitude.

Les fonctions occupées par ces salariés étaient réparties ainsi :

- 3 personnes étaient à la fois délégués du personnel et élus au comité d'entreprise;

- 1 personne était à la fois délégué du personnel et membre du comité d'hygiène de sécurité et des conditions de travail;

- 1 personne était à la fois élu au comité d'entreprise et membre du comité d'hygiène de sécurité et des conditions de travail;

- 2 personnes étaient à la fois délégués syndical et élus au comité d'entreprise (1) ou membre du comité d'hygiène de sécurité et des conditions de travail (1); 
- 5 personnes ne détenaient qu'un seul mandat : délégué syndical (2), délégué du personnel (1), membre du comité d'hygiène de sécurité et des conditions de travail (1) , conseiller prud'homme (1).

Seuls 7 d'entre eux étaient syndiqués et 5 étaient non syndiqués.

\section{Le processus conflictuel comme clef de compréhension sociologique}

L'objet de l'étude est moins le licenciement lui-même que l'augmentation des demandes de licenciements de salariés protégés par l'entreprise ${ }^{1}$ ainsi que la plus grande "acceptation" par les salariés protégés eux-mêmes ${ }^{2}$. Il en résulte que la question de l'existence ou non d'un conflit autour de la décision de licencier devient, dans une telle configuration, centrale. À cet, égard, il apparaît important de distinguer le désaccord du conflit et du litige. Si les frontières apparaissent ténues, ces termes doivent être différenciés dès lors qu'ils recouvrent différentes phases du processus de licenciement des salariés protégés.

Si la définition du "conflit" en termes de "relation antagonique entre deux ou plusieurs unités d'action dont l'une au moins tend à dominer le champ social de leurs rapports" (Bourricaud, 2002)" permet de comprendre la logique des conflits elle ne permet pas de caractériser les relations comme conflictuelles ou non.

Appliqué au champ du travail, le terme de conflit recouvre la cessation du travail, plutôt qu'une relation entre deux personnes ou deux entités ${ }^{3}$. Dès lors que la notion s'inscrit dans une dynamique, (il en va de même pour d'autres notions telles que la crise), la question de savoir à quel moment ou quelle est la frontière du phénomène analysé ne peut recevoir de réponse franche.

En sociologie, et particulièrement en sociologie du travail, la notion de conflit est appréhendée sous l'angle du conflit collectif ${ }^{4}$. Parce que les salariés protégés sont intégrés dans une logique statutaire, le conflit est régi par des règles qui, de prime abord, se réfèrent au fonctionnement collectif de la représentation des salariés. Mais parce sont visés des salariés en tant que personne, la dimension individuelle est également prégnante et parfois même dominante.

Dans le cadre de notre étude, nous utiliserons les notions de processus conflictuel plutôt que de conflit en entendant par processus "la dynamique d'un ensemble d'éléments articulés dans une constellation. Ces éléments sont des activités, des actions, des opérations [...] ou des relations [...]. Ces activités et ces relations induisent des effets d'ordre cognitif ou socio-

\footnotetext{
${ }^{1} 15265$ salariés protégés ont fait, en 1999, l'objet d'une demande de licenciement, tous motifs confondus qui correspond à une augmentation de 20\% par rapport à l'année précédente (entre 1999 et 2000 par contre on constate une diminution de 4\%). L'augmentation a d'abord démarré entre 1990 et 1993 puis à partir de 1998. Merlier R., "Les licenciements de représentants du personnel en 2000", Premières informations, novembre 2002, n 48.2.

${ }^{2}$ Le taux de recours par des salariés qui contestent une autorisation de licencier oscille entre $1 \%$ et $2 \%$, alors que ce taux est de presque un tiers en 1999 pour les employeurs qui contestent un refus de licencier. Merlier R., "Les licenciements de représentants du personnel en 2000", Premières informations, novembre 2002, n 48.2.

${ }^{3}$ C'est par exemple cette définition qui est utilisée pour évaluer les conflits du travail au niveau statistique. Brochard D. (2003), "Evaluation des statistiques administratives du travail", Document d'études n79, nov. 2003, DARES

${ }^{4}$ Que le traité de sociologie, coordonné par Raymond Boudon (1992), qui prône l'individualisme méthodologique, ne traite, dans le chapitre conflits rédigé par Pierre Birnbaum, que des conflits collectifs ou du conflit social, selon l'expression utilisée par l'auteur, donne idée de la difficulté pour la sociologie à appréhender le conflit inter-individuel. Boudon R (dir.). (1992), "Traité de sociologie du travail", PUF).
} 
affectif. L'ensemble de ces activités, de ces relations et de leurs effets constitue une constellation. [...] Cette constellation d'éléments est dynamique, dans la mesure, précisément, où les effets induits sont eux-mêmes inducteurs, où le mouvement d'ensemble se nourrit des effets qui s'y produisent, des transformations cognitives et socio-affectives qu'il engendre." (Charlot,\& alii, 1992)

Dès lors, dans notre analyse des processus de licenciement, nous allons nous intéresser plus à la dynamique d'ensemble qu'à un état. Dans cette perspective le conflit ne peut pas être expliqué par des causes simples et univoques. Il est alors possible de définir le processus conflictuel de manière pragmatique comme une divergence importante entre les acteurs sociaux, qui se trouve, à un moment donné, symbolisée par le dépôt d'une demande de licenciement à l'encontre du salarié protégé.

Ce processus débute par la survenance d'événements déclencheurs. En effet, l'émergence d'un conflit, ou le passage à une phase exacerbée de l'affrontement, ne survient que lorsque la configuration relationnelle entre salariés protégés et entreprise se modifie. Ces changements peuvent avoir plusieurs causes. Il peut s'agit notamment de changements de modes de management : les changements de direction ou de lignes managériales constituent des phases clés dans l'origine des conflits. C'est alors de nouveaux modes d'organisation qui, perçus par le salarié comme une remise en cause personnelle, voire une agression, interviennent de manière déterminante dans la décision d'entrer soit "dans le mandat", soit dans une opposition.

Compte tenu de la présentation que nous venons d'en faire, le conflit n'est bien sûr pas vu uniquement sous l'angle sociologique, il intègre l'analyse de la subjectivité de chaque acteur. Toutefois, nous mobiliserons aussi, par la suite, les apports des sociologues et en particulier ceux de Simmel qui distingue divers types de conflits, par exemple ceux qui revêtent une dimension purement interpersonnelle de ceux qui présentent un caractère plus objectif dans la mesure où les acteurs apparaissent cette fois comme les représentants de "causes" collectives. Cette distinction est ici primordiale non seulement parce que, en théorie, parler de représentants des salariés suppose de s'intéresser au collectif, mais aussi et surtout parce qu'elle fournit une grille de lecture tout à fait opérationnelle pour l'analyse des configurations relationnelles qui se nouent au moment des demandes de licenciement.

\section{Plan de lecture}

On peut ainsi aborder le licenciement des salariés protégés en utilisant une analyse séquentielle, chaque séquence faisant l'objet d'une analyse en tant que moment d'un processus.

En premier lieu, il faut analyser la situation au moment de l'élection ou de la désignation, avant le licenciement donc, qui explique le licenciement lui-même, mais aussi ses formes ou les motifs Nous structurerons l'analyse en nous centrant sur l'occupation du mandat qui fera l'objet de notre première partie.

Dans la deuxième partie, nous tenterons de prendre de la distance par rapport aux raisons et positions de ces deux acteurs que sont l'entreprise et le salarié protégé pour s'intéresser à la manière dont les autres protagonistes, et principalement l'inspecteur du travail, tiers institutionnel, interviennent et éventuellement influent sur le conflit. 
Enfin, dans la dernière partie, nous nous intéresserons à la manière dont les protagonistes parviennent ou non à sortir du conflit suite à la demande de licenciement : du refus d'une négociation et la poursuite de procédures de recours, ou d'acceptation éventuellement négociée. 


\section{I $^{\text {ERE }}$ PARTIE - L'OCCUPATION DU MANDAT PAR LE SALARIE PROTEGE : ANALYSE DES LOGIQUES A L'CEUVRE}

Si le mandat du salarié protégé s'exerce dans un cadre juridique contraignant qui contribue à modeler les comportements (I), il ne s'y limite cependant pas. L'occupation du mandat est marquée par différentes configurations dépendantes de la conception qu'a le salarié protégé de la relation de travail (II) qui tend cependant à évoluer vers une plus forte personnalisation (III).

\section{I- L'existence d'un cadre juridique contraignant}

Le droit occupe une place fondamentale dans le déroulement d'une procédure qu'il convient d'appréhender dans son sens le plus large, c'est-à-dire dès la survenance des premiers désaccords qui conduiront éventuellement l'entreprise à déposer une demande de licenciement auprès de l'inspecteur du travail. En réglant, par avance, dans un statut juridique dérogatoire au regard des autres salariés de l'entreprise non investis de mandats, la situation des salariés protégés, c'est-à-dire sous mandat (A), le droit trace certains contours aux conflits nés ou à naître. Ce faisant, il impose le respect d'un corpus de règles bâties autour de droits et devoirs opposables, c'est-à-dire dont peuvent se prévaloir les parties : le mandat produit alors certains effets (B). Le droit vient alors renforcer la capacité de l'une ou de l'autre partie à imposer ses propres objectifs, en fonction d'un "rapport de force juridique" que se construit l'issue du conflit.

\section{A) Le salarié protégé sous mandat}

Le droit apparaît aujourd'hui comme un cadre contraignant, pour l'entreprise mais aussi parfois pour le salarié. Il confère au salarié protégé une protection étendue (1) attachée au mandat et non à la personne du salarié protégé (2).

\section{1) Une protection étendue}

La protection instituée par le Code du travail vise ceux que l'on regroupe sous la dénomination de "salariés protégés", catégorie extrêmement hétérogène qui comprend :

- les représentants du personnel, stricto sensu, dans l'entreprise : délégués du personnel ${ }^{1}$, membres du comité d'entreprise ${ }^{2}$, représentants syndicaux auprès des comités d'entreprises, représentants des salariés aux conseils d'administration ou de surveillance des entreprises soumises à la loi de démocratisation du secteur public ; membres des comités d'hygiène, de

\footnotetext{
${ }^{1}$ Article L425-1.

${ }^{2}$ Article L436-1.
} 
sécurité et des conditions de travail $(\mathrm{CHSCT})^{1}$; représentants des salariés des entreprises en redressement judiciaire, délégués à la délégation unique du personnel depuis $1994^{2}$.

- mais également des salariés chargés de défendre les droits des travailleurs (conseillers prud'homaux $^{3}$ dès lors qu'ils sont entrés en fonction ${ }^{4}$ ),

- ou leurs intérêts (conseillers du salarié depuis 1991),

- ou de représenter leur syndicat auprès de l'entreprise (les délégués syndicaux) ${ }^{5}$

- et encore les salariés chargés de négocier des accords (salariés mandatés depuis 1998) dont on peut se demander s'ils représentent l'intérêt des salariés de l'entreprise et/ou de l'organisation syndicale, voire leur intérêt.

- les médecins du travail depuis la loi de modernisation sociale du 17 janvier $2002^{6}$.

Le droit regroupe donc sous une même catégorie bénéficiant de conditions de protection proches $^{7}$, dès lors qu'ils possèdent la qualité de salarié protégé ${ }^{8}$, des mandats cependant très différents, notamment par les enjeux qu'ils véhiculent. Un délégué syndical ou un salarié mandaté chargé de négocier les accords de réduction du temps de travail se trouve ainsi plus exposé aux critiques et aux attaques de l'employeur mais aussi, parfois, des salariés euxmêmes, mécontents du contenu des accords négociés. À l'inverse, un mandat dans un CHSCT se révèle plus consensuel, du fait de la nature des questions abordées (la santé au travail); le médecin du travail, dont le rôle est loin d'être négligeable, n'a pas pour fonction de représenter les salariés.

De la même manière, la perception subjective du mandat qu'a l'employeur ou le salarié varie suivant qu'il s'exerce dans ou hors de l'entreprise. Les contraintes divergent, tant pour le salarié que pour l'employeur.

Sur le strict plan de l'analyse juridique, une telle protection, conférée par la loi, apparaît très étendue au regard de la situation des autres salariés. L'autorisation administrative préalable s'impose, selon la jurisprudence, pour toute cause de rupture du contrat de travail, qu'il s'agisse d'un licenciement, d'une mise à la retraite ou encore d'une fin de contrat à durée déterminée (CDD) : suivant le Conseil d'Etat, "l'arrivée du terme du contrat n'entraîne

\footnotetext{
${ }^{1}$ Art. L. 236-11 du Code du travail, qui se renvoie aux articles L. 436-1, L. 436-2 et L. 436-3.

2 La délégation du personnel au CE (délégation unique du personnel) a été instituée par la loi quinquennale relative au travail, à l'emploi et à la formation professionnelle du 20 décembre 1993. Il s'agit d'adapter la représentation du personnel dans les PMPE ; dans les entreprises comprenant entre 50 et 200 salariés, il est possible de n'élire qu'une seule délégation qui reçoit les attributions du CE et des DP.

${ }^{3}$ Article L514-2 du Code du travail.

${ }^{4}$ Comme tout autre magistrat, un conseiller prud'homme ne peut être considéré comme étant en fonctions qu'après avoir prêté serment et avoir été installé dans ses fonctions. En conséquence, dès lors que ces deux dernières conditions ne sont pas remplies à la date du licenciement, un conseiller prud'homme ne peut bénéficier de la protection établie par l'article L. 514-2 du Code du travail, alors en vigueur, selon lequel le licenciement d'un conseiller prud'homme salarié en fonctions ou ayant cessé l'exercice de ses fonctions depuis moins de six mois, ne peut intervenir que sur décision du bureau de jugement présidé par le président du tribunal de grande instance dans le ressort duquel est situé le siège du conseil de prud'hommes. Cass. Soc. 16 juin $1988-\mathrm{N}^{\circ} 369$.

${ }_{6}^{5}$ Article L412-18 du Code du travail.

${ }^{6}$ Article L241-6-2 du Code du travail.

${ }^{7}$ L'autorisation de l'inspecteur du travail est requise. La consultation du CE peut être un préalable (mandats de membre du $\mathrm{CE}$, de $\mathrm{DP}$, médecin du travail). L'étendue de la période de protection à l'issue du mandat varie également.

${ }^{8}$ La chambre criminelle de la Cour de cassation a ainsi reconnu que l'exercice d'activités syndicales n'impliquait pas nécessairement la qualité de délégué syndical et donc de salarié protégé. Cass.Crim. 29 octobre 1996 - №379: "Attendu qu'en l'état de ces énonciations, dépourvues d'insuffisance, l'arrêt attaqué n'encourt pas les griefs allégués; que l'exercice d'une activité syndicale n'implique pas nécessairement la qualité de délégué syndical; que, par ailleurs, s'il est vrai qu'en exerçant les attributions réservées aux seuls représentants du personnel, le salarié, qui n'était investi d'aucun mandat, a outrepassé ses droits, cet excès de pouvoirs, en relation directe avec l'activité syndicale de l'intéressé, ne constitue pas un abus caractérisé débordant le cadre de l'exercice normal de celle-ci; qu'enfin, les juges, qui n'avaient pas à répondre mieux qu'ils ne l'ont fait aux conclusions qui leur étaient soumises, ont souverainement estimé que, pour arrêter sa décision, l'employeur avait pris en considération l'activité syndicale de l'intéressé".
} 
la cessation du lien contractuel qu'après constatation par l'inspecteur du travail, saisi dans les conditions prévues à l'article L. 436-1, que le salarié ne fait pas l'objet d'une mesure discriminatoire. Un mois avant l'arrivée du terme du contrat, l'employeur doit saisir l'inspecteur du travail qui doit statuer avant la date du terme du contrat"1.

D'autre part, aucune modification des conditions de travail du salarié protégé ne peut être réalisée sans son accord. Il est en effet de jurisprudence constante qu' "aucune modification de son contrat ou de ses conditions de travail ne pouvant être imposée à un salarié protégé, il appartient à l'employeur, en cas de refus du salarié, d'engager une procédure de licenciement, sauf manifestation de volonté non équivoque du salarié de démissionner" ${ }^{2}$. La modification des conditions de travail doit alors être, selon l'interprétation retenue par les juges, compatible avec "les exigences propres à l'exécution normale du mandat" ${ }^{\prime 3}$. On peut également noter que si le salarié protégé est fondé, en présence d'une inexécution fautive, par l'employeur, de ses obligations contractuelles, à prendre l'initiative de la rupture du contrat, cette dernière doit cependant s'analyser en un licenciement qui impose à l'employeur le respect des dispositions protectrices. Son accord est nécessaire dans tous les cas de figure, quand bien même le salarié protégé serait-il soumis, dans son contrat de travail, à une clause de mobilitét ${ }^{4}$ Le transfert d'un salarié vers une autre entreprise, réalisé en vertu de l'article L.122-12, est soumis à l'autorisation préalable de l'inspecteur du travail ${ }^{5}$.

Cette protection, qualifiée d'exceptionnelle et exorbitante du droit commun par les juges ${ }^{6}$, interdit à l'employeur de poursuivre, par d'autres moyens, la rupture du contrat de travail. Par conséquent, le respect des formalités protectrices s'impose, à peine de nullité, en cas de licenciement (motif économique ou personnel ${ }^{7}$ ), en cas de mise à la retraite ${ }^{8}$, de rupture d'un commun accord $^{9}$, de transaction ${ }^{10}$, et de transfert du salarié protégé en vertu de l'article L. $122-12,2^{\mathrm{e}}$ alinéa du Code du travail ${ }^{11}$.

Le droit vise, au travers d'une procédure contraignante, le maintien dans l'emploi du salarié protégé investi d'un mandat: licencié sans autorisation, il doit être réintégré. C'est ainsi que la Cour de cassation impose à l'employeur qui s'est vu refuser l'autorisation de licencier un délégué, de le mettre "non seulement à même d'exercer ses attributions représentatives, mais encore et d'abord d'être réintégré dans son emploi antérieur", la violation de cette obligation constituant "une atteinte punissable aux prérogatives statutaires du délégué"12, "qu'en effet, un délégué du personnel, pour remplir sa fonction, doit travailler effectivement dans l'entreprise" ${ }^{13}$. Le lien est donc clairement affirmé entre l'exercice du mandat et la présence sur le lieu de travail.

\footnotetext{
${ }^{1}$ Conseil d'Etat, octobre 1997, Yacco.

${ }^{2}$ Cass. Soc. 30 juin 1993, D. 1994, somm. 299, obs. Verdier ; Cass. Soc. 10 mai 1999, SA Saison dorée RJS n 830

${ }^{3}$ CE 27 juin 1997, Valadou.

${ }^{4}$ Cass. Soc. 23 septembre 1992 - N 477, société Lyon parc auto c/ Besse.

${ }^{5}$ Articles L. 412-18 pour les DS ; L 425-1, $6^{\mathrm{e}}$ al. ; 436-\&, $5^{\mathrm{e}}$ al pour les DP et membres du CE.

${ }^{6}$ Soc. 4 avril $1990-\mathrm{N}^{\circ} 159$.

${ }^{7}$ Il s'impose même en cas de faute lourde (Cass. Soc. 19 février 1981, Bull. civ. V, n 145).

${ }^{8}$ Soc. 5 mars 1996, Dr. Soc. 1996, p. 537, obs. Cohen ; CE 8 février 1995 - N 134963, Ministre du travail, de l'emploi et de la formation professionnelle et Crédit Lyonnais.

${ }^{9}$ Soc. 21 février 1996, Dr. Soc. 1996, p. 640, note Cohen.

${ }^{10}$ Cass. Soc. 10 juillet 2002, RJS 2002, $\mathrm{n}^{\circ} 1255$ : toute transaction conclue entre l'employeur et le salarié protégé avant la notification de son licenciement prononcé après autorisation administrative est atteinte d'une "nullité absolue d'ordre public". 11 "S'il survient une modification dans la situation juridique de l'entreprise de l'employeur, notamment par succession, vente, fusion, transformation du fonds, mise en société, tous les contrats de travail en cours au jour de la modification subsistent entre le nouvel employeur et le personnel de l'entreprise".

${ }^{12}$ Cass. Crim. 11 juin 1974, Baudry, cité par Touffait, p. 602.

${ }^{13}$ Cass. Crim. 3 juillet 1968, Meyer, D. 1969, p. 597 à 601, note J.-M. Verdier.
} 
La méconnaissance du statut protecteur des salarié protégé est pénalement répréhensible ${ }^{1}$. Lorsque le licenciement d'un salarié protégé n'a pas été autorisé par l'inspecteur du travail, le salarié protégé doit retrouver son emploi, le juge des référés pouvant prononcer la réintégration $^{2}$. La force coercitive, que l'on pourrait penser incitative, du droit ne suffit pourtant pas à asseoir le mandat dans les pratiques sociales. Si dans certaines entreprises étudiées les représentants du personnel sont des partenaires incontournables, ils peuvent aussi apparaître, dans d'autres, comme un obstacle.

\section{2) La protection est attachée au mandat et non à la personne}

La protection qualifiée d'"exceptionnelle" par le Conseil d'Etat ${ }^{3}$ dont bénéficient les salariés protégés apparaît, au travers de l'analyse du droit, et notamment de la jurisprudence, attachée au mandat et non à la personne. C'est du moins ce qui ressort de l'analyse tant du droit français qu'international ou communautaire ${ }^{4}$

Le lien juridique établi, au fil des jurisprudences, entre le mandat et le travail a permis de fonder, en droit, une certaine objectivation du mandat : il s'agit moins de protéger la personne que les fonctions qu'elle assume. C'est l'idée que l'on retrouve développée dans la circulaire du Ministère du travail : "il convient de rappeler que la discrimination doit être appréciée eu égard à l'exercice du mandat du représentant du personnel ; des conflits de personnes ou des traitements différenciés, mais sans lien direct avec l'activité du représentant du personnel ne sont pas suffisants pour retenir ces motifs" .

C'est parce que la protection est liée au mandat, dans sa dimension objective, qu'elle s'applique alors même que le salarié protégé n'a jamais exercé ses fonctions, la protection étant, selon la Cour de cassation, "indépendante de l'accomplissement de missions dont la mise en œuvre ne lui appartient pas"6 ${ }^{1}$. De la même manière, encourt l'annulation la décision de l'inspecteur du travail qui a été prise alors même que ce dernier n'avait pas connaissance de la totalité des mandats détenus; il n'était alors pas en mesure d'apprécier si des motifs d'intérêt général rendaient opportun le licenciement ${ }^{7}$. Il s'agit, au travers du droit, de garantir l'exécution normale, c'est-à-dire telle qu'elle a été instituée par le Code du travail, du mandat. C'est ce qui fonde la Cour de cassation à énoncer que l'exercice du mandat ne peut donner lieu à aucune perte de rémunération et que le temps passé doit être assimilé à du temps de

\footnotetext{
${ }^{1}$ La chambre criminelle de la Cour de cassation a ainsi pu décider que "Le licenciement d'un salarié protégé effectué en méconnaissance de la procédure légale constitue le délit d'entrave; la rétractation de l'employeur n'est pas de nature à faire disparaître l'infraction" ; Cass. Crim. 4 avril $1995-\mathrm{N}^{\circ} 146$.

${ }^{2}$ Cass. Soc. 12 juin $2001-\mathrm{N}^{\circ} 220$.

${ }^{3}$ Conseil d'Etat, 15 juin 1998, Onet Propreté : "Considérant qu'en vertu de l'article L. 412-18 du code du travail, les délégués du personnel, qui bénéficient, dans l'intérêt de l'ensemble des travailleurs qu'ils représentent, d'une protection exceptionnelle"

${ }^{4}$ CONSEIL DE L’EUROPE : charte sociale européenne signée le 18 oct. 1961 à Turin et modifiée le 3 mai 1996: "Les représentants des travailleurs dans l'entreprise ont droit à la protection contre les actes susceptibles de leur porter préjudice et doivent avoir les facilités appropriées pour remplir leurs fonctions (art. 28).

UNION EUROPEENNE : "Les États membres veillent à ce que les représentants des travailleurs jouissent, dans l'exercice de leurs fonctions, d'une protection et de garanties suffisantes leur permettant de réaliser d'une façon adéquate les tâches qui leur ont été confiées" (Directive 2002/14 du 11 mars 2002 sur l'information et la consultation des travailleurs, article 7). CONSEIL CONSTITUTIONNEL FRANÇAIS (décision du 20 juillet 1988) : «les représentants du personnel bénéficient d'une protection spécifique "découlant d'exigences constitutionnelles" et justifiées "par les difficultés que présente l'exercice de leurs fonctions"

${ }^{5}$ DRT nº 96-11 du 3 décembre 1996, Ministère du travail et des affaires sociales, Dr. Ouvrier mai 1997, p. 201 à 207, p. 205.

${ }^{6}$ Cass. Soc. 14 janvier 2003, Boulangerie de l'Europe c/ N. Bakhti, Dr. Soc. 2003, p. 445 à 447, obs. F. Duquesne

${ }^{7}$ CE 22 juillet 1992, Girelli ; 4 mars 1996, Sté Maf Plastique. Mais ce n'est pas le cas si l'inspecteur a été informé au cours de son instruction Circulaire DRT $n^{\circ} 3$ du 1er mars 2000, Ministère de l'emploi et de la solidarité, Dr. Ouvrier oct. 2000, p. 442 à 451
} 
travail ${ }^{1}$. L'indépendance, notamment économique, du salarié protégé participe à l'exercice normal de mandat. C'est sur cette même logique que le Conseil d'Etat s'appuie pour annuler la décision d'un inspecteur du travail autorisant le licenciement d'un salarié protégé motivée par l'accord donné par le salarié, s'appuyant sur « les exigences propres à l'exécution normale de son mandat $»^{2}$.

C'est donc le mandat et, au travers du mandat, l'instance représentative du personnel en tant que lieu d'expression collective des salariés qui est l'objet de la protection, et non la personne qui l'occupe. C'est pourquoi le salarié protégé licencié sans autorisation préalable de l'inspecteur du travail ou en dépit du refus de ce dernier a droit à une indemnisation spécifique forfaitaire ${ }^{3}$ due au titre de la méconnaissance du statut protecteur mais également aux indemnités de droit commun versées à tout salarié en cas de licenciement prononcé sans cause réelle et sérieuse ${ }^{4}$. Ainsi que le remarque très justement J.-Y Frouin ${ }^{5}$, "en d'autres termes, il y a autonomie du statut protecteur et du contrat de travail : la violation du statut est sanctionnée par l'obligation faite à l'employeur de payer la rémunération jusqu'à la fin de la période de protection [aujourd'hui plafonnée] puisque le licenciement intervenu n'a pu produire aucun effet; mais le contrat de travail étant malgré tout rompu, faute de réintégration, cette rupture est appréciée conformément au droit commun du licenciement".

On peut cependant s'interroger sur les effets juridiques produits par ce statut qui peut parfois conduire, pour une même situation, à une différence de traitement entre salariés protégés et non protégés. Comment s'opère le contrôle juridictionnel ? L'appréciation donnée par les juges est-elle la même ou bien existe-t-il une différence de traitement légitimée par le statut juridique dérogatoire?

Suivant un arrêt de principe du Conseil d'Etat daté du 29 décembre 2000, le salarié protégé ne bénéficie pas d'une priorité de reclassement sur les autres salariés : "il n’y a pas lieu de rechercher la possibilité du reclassement du salarié protégé sur un poste dont la libération n'implique pas l'éviction d'un autre salarié de l'entreprise ${ }^{6}$. Le salarié protégé ne bénéficie donc pas, au plan de l'analyse juridique, d'une garantie d'emploi. On note cependant que la mise en application des textes par la jurisprudence conduit, s'agissant de la modification apportée par l'employeur aux conditions de travail du salarié protégé, à une différence de traitement. Depuis un arrêt de principe de $1996^{7}$, la Cour de cassation a introduit, dans sa jurisprudence, une distinction entre modification du contrat de travail et modification des conditions de travail, distinction ${ }^{8}$ qui a pour effet de régir la situation juridique des salariés

\footnotetext{
${ }^{1}$ Cass. Soc. 19 mars 2002, Semaine soc. Lamy som. $n^{\circ} 1069$.

${ }^{2}$ CE $1^{\text {er }}$ fév. 1995, SA Midica.

${ }^{3}$ Le salarié protégé a droit, "au titre de la méconnaissance du statut protecteur", au montant de la rémunération qu'il aurait dû percevoir entre son éviction et l'expiration de la période de protection, dans la limite de la durée de la protection accordée aux représentants du personnel (Cass. Soc. 27 mai 1970, Abisse, Dr. Soc. 1971, p. 130, Dalloz 1970, P. 442 Chron. Dupeyroux ; Cass. Soc. 28 mars 2000, n 97-44.373, Semaine soc. Lamy, 31 juillet 2000, n 992, p. 61 à 62).

${ }^{4}$ Art. L. 122-14-4 (au moins 6 mois de salaire).

${ }^{5}$ Frouin (J.-Y), "L’indemnisation des salariés protégés licenciés sans autorisation", RJS 11/01, p. 842 à 848, p. 844.

${ }^{6}$ Conseil d' Etat $N^{\circ} 206918$ et n ${ }^{\circ} 206919,29$ décembre 2000, société Suturex: "il appartient à l'inspecteur du travail et, le cas échéant, au ministre, de rechercher, sous le contrôle du juge de l'excès de pouvoir, si la situation de l'entreprise justifie le licenciement du salarié, en tenant compte, notamment, de la nécessité des réductions d'effectifs envisagées et de la possibilité d'assurer le reclassement du salarié dans l'entreprise ; qu'il y a lieu, à cet égard, de rechercher la possibilité du reclassement du salarié protégé sur un poste dont la libération n'implique pas l'éviction d'un autre salarié de l'entreprise".

Conseil d'Etat 8 octobre 1990, M. DEIAS : "les dispositions de l'article L. 241-10-1 du code du travail n'obligeaient pas la société Bennes Marrel à libérer un de ces emplois au profit de M. DEIAS ou à créer un nouvel emploi qui aurait pu convenir aux aptitudes physiques de l'intéressé".

${ }^{7}$ Cass. Soc. 10 juillet 1996, Le Berre C / SA Socorem.

${ }^{8}$ Distinction qui s'est substituée à l'ancienne distinction qui existait entre modification substantielle / non substantielle.
} 
dans l'entreprise : s'ils ne peuvent, conformément à cette jurisprudence, se voir imposer une modification de leur contrat sans leur accord (l'employeur qui persiste devant alors avoir l'initiative du licenciement), ils ne peuvent, sous peine de commettre une faute grave, refuser une modification de leur conditions de travail. Or, les salariés protégés sont juridiquement fondés à refuser toute modification, quelle que soit sa nature. Il en résulte des différences de traitement en des circonstances identiques. C'est ainsi que la mise en chômage partiel, qui, selon la Cour de cassation, "doit s'analyser en une simple modification des conditions de travail et non du contrat" conduit à ce que le refus des salariés non protégés ne peut s'analyser en une rupture de leur contrat (ils ne peuvent prétendre être indemnisés des pertes de salaires subies du fait du chômage partiel) tandis que le représentant du personnel doit se voir verser par l'employeur la partie du salaire perdue dès lors qu'aucun changement de ses conditions de travail ne peut intervenir sans son accord ${ }^{1}$.

De la même manière, la qualification juridique donnée par le Ministre du travail, sur recours hiérarchique contre la décision de l'inspecteur du travail, à une faute commise au cours d'une grève (la séquestration d'un directeur commercial par des salariés, dont certains étaient protégés, constitue-elle une faute lourde autorisant le licenciement sans versement d'aucune indemnité ?) ne lie pas le juge judiciaire. La décision du Ministre de refuser le licenciement de quatre salariés protégés ne peut ainsi valablement intervenir à l'appui de la demande de salariés non protégés contre lesquels la Cour de cassation retient, pour sa part, l'existence d'une faute grave ${ }^{2}$.

Une telle différence de traitement, parfois accentuée par la dualité contentieuse ${ }^{3}$, peut, si elle n'est pas clairement fondée sur l'existence d'un mandat qui la "légalise" tout en la légitimant, apparaître comme une source de discrimination à rebours. Il n'en demeure pas moins que le contexte social peut également jouer un rôle dans la prise de décision du Ministre, rendue sur recours hiérarchique. La décision ministérielle intègre parfois des éléments d'appréciation tels que la volonté de pacifier des relations sociales, auxquels sont moins sensibilisés les juges. Il se peut ainsi que les appréciations portées par le Ministre et par les juges divergent pour une même situation.

\section{B) Les effets de ce mandat}

Le mandat confère à celui qui l'occupe certaines prérogatives (1) qui participent de la construction d'un statut social du salarié protégé (2). Ce faisant, le droit a institué une relation juridique duale: le salarié protégé se situe à la fois sous contrat et sous statut, lesquels obéissent à des logiques juridiques différentes (3).

\footnotetext{
${ }^{1}$ Cass. Soc. 18 juin 1996, deux arrêts, CSB 1996, nº 84, A. 54, note.

${ }^{2}$ Cour de Cassation, soc., 1 avril 1997, $\mathrm{N}^{\circ}$ de pourvoi : 95-42246 $\mathrm{N}^{\circ}$ de pourvoi : 95-42264, Publié au bulletin

${ }^{3} \mathrm{Cf}$. Infra, IIIe partie sur l'issue de la procédure.

En vertu du principe de la séparation des pouvoirs seuls les tribunaux de l'ordre administratif ont compétence pour statut sur la légalité d'une décision administrative, les tribunaux judiciaires intervenant alors dans le cadre de la réparation. Les tribunaux judiciaires doivent ainsi surseoir à statuer dans l'attente de la décision de la juridiction administrative (Cass. soc. 3 juillet $1985-\mathrm{N}^{\circ}$ 391). Les juridictions judiciaires sont tenues, sur le fond, par les décisions de la juridiction administrative (Cass. Soc. 12 mars $1987-\mathrm{N}^{\circ} 149$ ). Le juge judiciaire ne peut, en l'état de l'autorisation administrative accordée à l'employeur de licencier les salariés protégés et sans violer le principe de la séparation des pouvoirs, apprécier le caractère réel et sérieux du motif de licenciement y compris en ce qui concerne le respect de l'obligation de reclassement. Il lui appartient seulement de surseoir à statuer en renvoyant l'appréciation de légalité à la juridiction administrative (Cass. Soc. 25 novembre 1997 - N 399).
} 


\section{1) Le mandat confère certaines prérogatives au salarié protégé}

Ces prérogatives octroyées par les textes sont étendues: la représentation des salariés implique, selon l'esprit des lois, la mise à disposition de moyens destinés à obtenir et/ou à diffuser l'information ou encore à transmettre des réclamations. Les textes positionnent, alternativement ou cumulativement, le salarié protégé dans un rôle d'opposition et/ou de contestation et/ou de proposition (lire l'encadré). Le Code du travail ${ }^{1}$ a même doté le délégué du personnel d'un droit d'alerter l'employeur de toute atteinte aux libertés, instituant une procédure contraignante. En effet, s'il constate lui-même ou par le biais d'un salarié, qu'il existe une atteinte aux libertés individuelles qui ne trouve pas sa justification dans l'article L. 120-2 $2^{2}$, le délégué du personnel doit saisir l'employeur qui est tenu de procéder sans délai à une enquête avec le délégué et de remédier à cette situation. En cas d'inaction de l'employeur ou de désaccord, le délégué saisit le Conseil de prud'hommes en référé, ce dernier pouvant ordonner toute mesure propre à faire cesser cette atteinte. Ce droit d'alerte n'est pas sans rappeler le droit d'alerte existant en cas de danger grave et imminent pour la sécurité physique des salariés et qui autorise le retrait du salarié de son poste de travail ${ }^{3}$.

Le Code du travail offre aux salariés protégés investis d'un mandat représentatif le pouvoir de "demander des comptes" à l'employeur sur sa gestion. C'est sur cet équilibre, ainsi que l'explique très justement Alain Supiot, que s'est bâti le droit du travail : "Dès lors que l'action des uns se trouve fondée par le droit de propriété et la liberté d'entreprendre, l'action des autres doit être garantie par un triple droit à la représentation, l'action et la négociation collective. C'est ce jeu de poids et de contrepoids entre action économique des entrepreneurs et action collective des salariés qui a constitué le véritable moteur du droit du travail et lui a permis tout à la fois de guider les transformations du monde du travail et d'être guidé par elles" ${ }^{4}$. C'est cette prise en compte de la nécessaire articulation entre des intérêts divergents visant la recherche d'un certain équilibre que traduit la Cour de cassation lorsqu'elle énonce que le pouvoir de direction dont dispose l'employeur, qui se traduit par le "droit d'organiser le travail dans son entreprise", doit se concilier avec d'autres impératifs ${ }^{5}$.

\footnotetext{
${ }^{1}$ Art. L. 422-1-1.

2 "Nul ne peut apporter aux droits des personnes et aux libertés individuelles et collectives de restrictions qui ne seraient pas justifiées par la nature de la tâche à accomplir ni proportionnées au but recherché". Article issu de la loi du 31 décembre 1992.

${ }^{3}$ Art. L. 213-9.

${ }^{4}$ Supiot (A), "Revisiter les droits d'action collective", Dr. Soc. 2001, p. 687 à 704, p. 687.

${ }^{5}$ Cass. Soc., $1^{\text {er }}$ avril 2003, $\mathrm{N}^{\circ}$ de pourvoi : 00-41873, Bulletin $2003 \mathrm{~V} \mathrm{~N}^{\circ} 125$ p. 121 : "Mais attendu que si, en application de l'article L. 122-28-1 du Code du travail, tout salarié dispose du droit, sous certaines conditions, soit de bénéficier d'un congé parental d'éducation durant lequel le contrat de travail est suspendu, soit de réduire sa durée de travail d'au moins un cinquième de celle qui est applicable dans l'établissement sans que cette activité à temps partiel puisse être inférieure à seize heures hebdomadaires, la fixation de l'horaire de travail, à défaut d'accord des parties, relève du pouvoir de direction de l'employeur ; que néanmoins le refus du salarié d'accepter les horaires proposés n'est pas constitutif d'une faute grave dès lors que la proposition de l'employeur n'est pas compatible avec des obligations familiales impérieuses ;

Et attendu que la cour d'appel a relevé, d'une part, qu'en l'absence d'accord, l'employeur avait fixé sans qu'il soit établi qu'il ait commis une faute dans l'exercice de son droit d'organiser le travail dans son entreprise, les horaires de travail de la salariée, et, d'autre part, que la salariée, qui s'est présentée à son travail un jour où elle devait être de repos tandis que le lendemain où elle aurait dû venir aussi bien selon son propre horaire qu'elle croyait devoir appliquer que selon celui demandé par l'employeur, et ne s'est pas présentée les jours suivants malgré la mise en demeure de l'employeur de reprendre le travail, n'apportait aucun élément permettant d'apprécier les contraintes invoquées à l'appui de son refus ; qu'en l'état de ces énonciations, la cour d'appel a pu décider que le refus réitéré de la salariée de reprendre le travail était de nature à rendre impossible le maintien de cette dernière dans l'entreprise pendant la durée du préavis et constituait une faute grave ; que le moyen n'est pas fondé".
} 
L'analyse des entretiens réalisés avec des salariés protégés montre que certains salariés s'emparent de ces prérogatives, comme cette déléguée du personnel à propos du plan de restructuration présenté par l'employeur :

\section{AVIS D'UNE DELEGUEE DU PERSONNEL SUR LE PLAN DE RESTRUCTURATION PRESENTE PAR L'EMPLOYEUR}

Mme X

Février 2003

Avis de déléguée du personnel "cadres" sur le plan de restructuration présenté

Messieurs,

Les éléments complémentaires, dans leur quasi-totalité, communiqués ce matin lors de notre réunion, n'ont pu faire l'objet d'une étude approfondie de ma part; aussi le contenu du présent document ne pourra pas être exhaustif dans les motifs évoqués pour vous faire part de mon opposition aux 8 suppressions de postes présentées.

Le fondement de ce plan est basé exclusivement sur un plan financier. Une restructuration des effectifs par suppressions de postes est présentée, et aboutit à une gestion par service avec une non-visibilité des compétences humaines du personnel nécessaire aux besoins d'un fonctionnement viable de l'entreprise. Seulement 6 salariés de l'entreprise ont été reçus pour discussion, fin novembre 2002. Les compétences des salariés de l'entreprise ne sont pas connues, et ce simple fait peut amener une nouvelle déstabilisation ultérieure suite à une non-évaluation préalable de celles-ci.

Les résultats présentés le sont dans un contexte où il est considéré que tout va pour le mieux afin de permettre un point de stabilisation par rapport à 2002 .

Les différents schémas évoqués ci-après ne présentent pas actuellement de solutions concrètes, mais restent en suspens et à l'état d'étude et d'estimation sans éléments de coûts réels

Les quelques réparations SAV actuellement externalisées font partie de l'existant quotidien de l'entreprise, rien de plus n'a été mis en place à ce jour malgré plusieurs réunions, ce problème n'est effectivement pas simple à gérer.

La médiocre qualité des composants cartes-mères, alimentations, etc.

Aucun système de dédommagement n'a été mis en place face à l'ampleur du problème des disques durs $\mathrm{F}$.

Il n'y a toujours pas plus de visibilité sur la qualité / fiabilité des délais de livraison et de fabrication de notre usine $\mathrm{W}$.

Les livraisons directes depuis l'Allemagne sont encore loin d'être performantes.

L'externalisation de la plate-forme logistique.

Le déménagement prévu de l'entreprise alors que le bail reste à courir encore 2 ans engendrerait d'autres grosses dépenses alors que l'entreprise a déménagé il y a moins d'un an avec tous les frais que cela engendre.

Les méthodes de remboursement des frais afférents à la vente des produits conceptronic (matériel humain) n'ont pas été mises en place

Pour tous ces points évoqués, non-exhaustifs cependant, et l'importante remise en question du fonctionnement général de l'entreprise, il semble nécessaire actuellement de conserver bon nombre des postes prévus à être supprimés, ces suppressions de postes me semblent excessives et prématurées compte tenu des objectifs fixés par votre plan de redressement et des moyens à mettre en place pour y parvenir, avec seulement 15 personnes (en comptant les 2 périodes d'essai non renouvelées). La mise en place des processus avec les compétences humaines actuelles me paraît être plus structurelle.

Pour ces raisons, je me prononce défavorablement à la procédure exposée.

Recevez, Messieurs, mes sincères salutations.

Mme X

Déléguée du Personnel "Cadres" 


\section{2) Le mandat confère un statut social au salarié protégé}

Le mandat est également source de reconnaissance en même temps qu'il participe de l'institution du statut social du salarié protégé. Il permet au salarié protégé d'acquérir, par le biais de ses fonctions, de nouvelles compétences, d'obtenir des informations par un accès privilégié à sa source et, ce faisant, de mieux connaître l'entreprise et d'en maitriser plus finement les rouages. Nous ne discutons pas ici de la légitimité des actions qui sont définies par la loi, les représentant du personnel sont dans leur rôle quand ils exigent le respect du droit. Pour autant le mandat permet aussi de faire valoir, voire d'opposer certaines compétences, en l'occurrence des compétences juridiques, à l'employeur.

\footnotetext{
"Jusqu'à preuve du contraire, il n'y a jamais eu de délégué d'entreprise, quand je suis rentré [dans l'entreprise], j'ai moi-même été délégué pendant 2 ans. Donc j'ai rétabli un peu une certaine discipline, une certaine situation, qui a fait qu'il y a quand même eu certains bénéfices pour les salariés : remise à jour des conventions collectives, remise à jour de leur application, c'est vrai qu'il y avait un laisser-aller de ce côté. Donc du fait que j'étais rentré [dans l'entreprise] en tant que délégué, en 2 ans, j'ai rétabli certaine situation, parce qu'il est vrai que j'étais conseiller prud'homme, donc proche des lois, des textes d'applications, proche des conventions collectives". [...] J'étais inscrit à la CGT, et ensuite le syndicat m'a demandé de monter, éventuellement, un syndicat d'entreprise, ce que j'ai fait, donc j'étais représentatif: je discutais avec les employeurs de façon à réinstaurer une certaine discipline au niveau du respect de la convention collective".» (Salarié, juge prud'homal, délégué syndical, ouvrier, 58 ans, CEP, TPE, demande de licenciement pour inaptitude)
}

Ce faisant, le mandat devient aussi une source de pouvoir non négligeable ainsi qu'en témoignent plusieurs salariés protégés.

\footnotetext{
"C'est moi qui dirigeais les 35h, et j'avais les 35 h dans les mains. À l'époque, il n'était pas encore question de tout ce qu'on entend maintenant, si on dénonçait l'accord des $35 \mathrm{~h}$, les aides tombaient, il fallait qu'ils renégocient au plus vite, et dans la mesure où ils savaient que les $35 \mathrm{~h}$ ils devaient les renégocier avec moi, ils étaient obligés de tout revoir parce que moi, c'était quelque part ma carte maîtresse. Parce qu'avec la renégociation des $35 \mathrm{~h}$, on peut tout faire, on peut revoir l'organisation du travail, la rémunération, on peut tout faire, donc c'était clair qu'à partir des $35 \mathrm{~h}$, moi je voulais qu'on mette tout sur la table..." (Salarié protégé, délégué syndical, cadre, 42 ans, Bac, PME, demande de licenciement pour faute)

\begin{abstract}
"Donc voilà, on arrive au comité d'entreprise avec les pleins pouvoirs parce qu'on était majoritaires, avec tout ce que cela suppose. Très rapidement, comme on était des gens un peu consciencieux et qu'on avait vraiment envie de faire bouger les choses, on s'est formé, on s'est beaucoup documenté, on a bossé comme des malades pendant 2 ans, et forcément, on a remué un peu la merde parce qu'on s'est rendus compte qu'il y avait des choses qui n'étaient pas nettes." ( Salarié protégé, membre du comité d'entreprise, délégué du personnel, syndiqué, 37 ans, cadre, $\mathrm{Bac}+2$, PME, demande de licenciement pour faute)
\end{abstract}

Ce pouvoir peut d'ailleurs parfois devenir extrêmement grisant pour celui qui en détient les clés, comme le décrit un salarié protégé cadre CGC, membre du comité d'entreprise.

"Et à partir du moment où on a commencé à se lancer là-dedans, très honnêtement, on s'est pris au jeu, c'est-à-dire que très rapidement, on s'est rendu compte des moyens qu'on pouvait avoir, de ce qu'on pouvait faire. Et vu ce qu'ils ont fait par la suite, ça prouve très clairement qu'on leur cassait les noix, on les enquiquinait sérieusement, et si on les enquiquinait sérieusement, ça prouvait bien 
qu'il y avait des choses qui n'étaient pas carrées et qu'on avait un certain pouvoir et qu'on pouvait changer les choses. Donc ça, ça nous intéressait beaucoup, donc on s'est franchement pris au jeu et on y a été à fond, comme des malades, et pendant 2 ans, on a bossé à temps complet pour le comité d'entreprise. Très honnêtement le magasin, on avait des heures de délégation et tout ça, déjà par principe on n'était pas souvent là, mais même quand on était en magasin... Moi, mon équipe, je l'ai prévenue tout de suite, je lui ai dit : bon maintenant je me lance là-dedans. D'ailleurs je l'ai fait pratiquement avec leur accord, je leur ai dit que je voulais me lancer là-dedans parce qu'il y avait des choses à changer, ils étaient d'accords avec moi, et je leur ai dit: est ce que vous êtes d'accords sur le fait que je vais être très souvent absent? Ils m'ont dit qu'il n'y avait pas de problèmes, de toute façon l'équipe tourne bien, il en reste encore 2 qui était là à l'ouverture, le $3^{\text {ème }}$ est là depuis plus de 3 ans maintenant, donc la boîte tourne, je sais que je peux leur faire confiance.» (Salarié protégé, membre du comité d'entreprise, délégué du personnel, syndiqué, 37 ans, cadre, Bac+2, PME, demande de licenciement pour faute)

Le statut juridique, et les prérogatives qu'il institue, confèrent certains pouvoirs qui sont parfois vécus, de l'avis du même salarié protégé, comme des remises en cause du pouvoir de l'employeur, ou, au minimum, qui participent à un certain rééquilibrage des positions entre dirigeants et salariés. En s'appuyant sur la législation qui leur confère des droits, les salariés protégés peuvent en partie inverser la situation de subordination, puisqu'ils sont à même d'imposer des contraintes à leurs dirigeants.

"C'est surtout une politique qui est basée sur la non-divulgation d'informations, c'est-à-dire que
les salariés ne doivent pas savoir comment fonctionne l'entreprise, ce n'est pas leur boulot. Donc à
partir de ce moment-là, à partir du moment où en tant que comité d'entreprise on veut connaître
parce que ça fait partie de notre travail de connaître comment fonctionne l'entreprise, comment est
géré la machine pour pouvoir le diffuser aux salariés dans une certaine mesure, lui ne supporte pas
ça, il ne supporte pas qu'on vienne mettre le nez dans ses affaires. Donc des gens comme nous,
pour lui, on est vraiment des fouilles merde, il ne supporte pas des mecs comme nous, c'est
viscéral. Rapidement son $1^{\text {er }}$ truc, ça a été d'essayer de nous dégager, il ne veut pas discuter avec
nous." (Salarié protégé, membre du comité d'entreprise, délégué du personnel, syndiqué, 37 ans,
cadre, Bac+2, PME, demande de licenciement pour faute)

La capacité des salariés protégés à résister et à s'opposer varie. On constate cependant que le droit est de plus en plus mobilisé, non seulement dans la gestion du litige mais également bien en amont. Il apparaît comme un "argument d'autorité" susceptible, dans la représentation qu'en donnent les salariés protégés interrogés, de s'imposer à l'autre partie.

\footnotetext{
"Je connais mes droits, je connais bien mes droits, c'est ce qui agaçait [l'entreprise] aussi. [...] Par exemple le jour où c'était inondé, où on travaillait, où il faisait 4 degrés, en plus il n'y avait que moi qui étais présent dans l'entreprise, je pouvais arrêter l'entreprise. C'est la seule fois où tous les salariés se sont mobilisés dans l'histoire de [l'entreprise], dans le sens où il y a un risque avec leurs conditions de travail, donc ils peuvent quitter leur poste et venir me voir parce qu'il y a un danger grave imminent [...] Les droits ont tout simplement commencé à être connus, à cause de moi, et ça, ça commençait à être très gênant pour eux.» (Salarié protégé, membre du comité d'entreprise et du CHSCT, syndiqué, agent de maîtrise, 28 ans, BTS, PME, demande de licenciement pour inaptitude)
}

Cette situation, quelque peu "ambivalente" dans le sens où le salarié protégé se sert, comme le montre l'exemple précité, du droit et de son statut comme moyen de s'opposer à son employeur et de faire valoir son point de vue, trouve son pendant, au plan de l'analyse juridique, dans l'existence d'une relation juridique duale : le salarié protégé se situe à la fois sous contrat et sous statut, lesquels obéissent à des logiques juridiques différentes. 


\section{3) Le salarié protégé sous contrat et sous statut}

Le lien contractuel qui unit le salarié protégé à l'entreprise se double d'un lien institutionnel, incarné par le statut sous lequel se trouve placé le salarié protégé : il est donc à la fois dans un lien de subordination, qui caractérise, entre autres critères, l'existence d'une relation de travail salariée, mais aussi sous statut, garant du plein exercice des missions pour lesquelles il a été élu ou désigné. Autrement dit, le salarié protégé est un salarié soumis à l'autorité de l'employeur mais disposant de certaines prérogatives ; il est lié à son employeur par contrat de travail tout en étant sous la protection d'un statut. Ce statut exorbitant du droit commun qui régit, pour partie, la relation de travail du salarié protégé tire son fondement juridique des missions qui incombent au salarié protégé ${ }^{1}$. Ainsi que l'explique clairement F. Duquesne, "à l'instar de tout salarié, le représentant du personnel est titulaire d'un contrat de travail qui fonde la subordination sous laquelle il se situe à l'égard de l'employeur. Mais le lien institutionnel le rattache, par la voie du suffrage ou de la désignation, à la collectivité des membres de l'entreprise" ${ }^{2}$. C'est parce qu'il représente la collectivité des travailleurs (directement ou indirectement, par le biais de l'organisation syndicale dans le cas du délégué syndical), qu'il doit bénéficier d'un statut exorbitant garantissant l'accomplissement de ses missions. Selon la Cour de cassation, "la protection exceptionnelle et exorbitante du droit commun des salariés investis de fonctions représentatives a été instituée non dans le seul intérêt de ces derniers mais dans celui de l'ensemble des salariés" ${ }^{3}$. Il en résulte que ce statut est, selon la consécration jurisprudentielle, d'ordre public absolu : il ne peut, par conséquent, y être dérogé ${ }^{4}$. Ce faisant, le statut inscrit le salarié protégé dans un temps qui n'est pas le seul temps de l'entreprise : il peut en effet, ainsi que l'a reconnu la Cour de cassation, continuer à produire des effets indirects même après la cessation de la période de protection ${ }^{5}$. De la même manière, le contrat de travail du salarié protégé se trouve suspendu durant le temps passé dans l'exécution de son mandat. Cette suspension soustrait donc le salarié au pouvoir de l'employeur, et notamment au pouvoir disciplinaire. Il est dispensé d'accomplir sa prestation de travail ${ }^{6}$ et peut refuser de collaborer avec lui ${ }^{7}$.

Mais c'est parce que le salarié dit «protégé » se trouve dans des liens contractuels que la Cour de cassation lui ouvre, depuis un récent arrêt très commenté et «estampillé » du sceau des arrêts particulièrement importants ${ }^{8}$, la possibilité de demander au juge la résiliation de son contrat de travail. En effet, tout en rappelant que la procédure de licenciement du salarié représentant du personnel est d'ordre public, la Cour de cassation décide « [...] qu'il ne peut être privé de la possibilité de poursuivre la résiliation judiciaire de son contrat de travail aux

\footnotetext{
${ }^{1}$ N. Maggi-Germain, "Statut et contrat: deux modes de construction de la relation de travail", La Revue de l'Ires, numéro spécial ( $\left.\mathrm{n}^{\circ} 45\right)$ Les relations professionnelles dans le secteur public, 2004/2, p. 103 à 117.

${ }^{2}$ F. Duquesne, "Représentants du personnel. Contrat de travail et exercice du mandat", RJS 7/02, p. 603 à 608, p. 603 ; J.-M. Berraud, "L'influence de la suspension du contrat de travail sur les relations institutionnelles dans l'entreprise, Dr. Soc. 1980, p. 151.

${ }^{3}$ Cass. Soc. 10 juillet 2002, RJS 2002, $\mathrm{n}^{\circ} 1255$.

${ }^{4}$ Cass. Soc. 23 mai 2000, $\mathrm{n}^{\circ}$ 97-42.145 P., Semaine sociale Lamy, 31 juillet 2000, $\mathrm{n}^{\circ}$ 992, p. 97

${ }^{5}$ Cass. Soc. 19 décembre $1990-\mathrm{N}^{\circ} 685$ : "En l'état de ses constatations selon lesquelles les motifs énoncés par l'employeur à l'appui du licenciement prononcé à l'expiration du délai de protection légale de l'ancien salarié protégé étaient les mêmes que ceux qui avaient été invoqués lors des demandes d'autorisation de licenciement adressées à l'autorité administrative pendant ce délai, une cour d'appel, nonobstant le fait que le licenciement ait été prononcé postérieurement à l'expiration de la période de protection, décide à bon droit que cette mesure revêtait un caractère abusif".

${ }^{6}$ Cass. soc. 15 juin 1999, Dr. soc. 1999, p. 842, obs. Mazeaud

${ }^{7}$ Seul le comportement déloyal sera sanctionné en période de suspension du contrat de travail (accomplissement d'une formation dans une entreprise concurrente ; cass. Soc. 10 mai 2001, RJS 7/01, n 833). S'agissant du salarié protégé, il devra s'agir d'une "acte totalement détachable de son mandat" (F. Duquesne, op. cit., p. 608).

${ }^{8}$ La Cour de cassation «classe » ses décisions suivant une certaine nomenclature : P (publié au Bulletin des arrêts de la Cour de cassation (édition mensuelle), B (publié, en résumé, au Bulletin d'information de la Cour (bimensuel), R (rapport annuel), I (site internet), NP (non publié).
} 
torts de l'employeur en cas de manquement, par ce dernier, à ses obligations ${ }^{1}$. Ce faisant, la Haute juridiction aligne le régime juridique applicable aux salariés dits «protégés » sur celui des salariés ordinaires. La résiliation prononcée aux torts de l'employeur produira les effets d'un licenciement sans cause réelle et sérieuse : l'initiative de la rupture incombe au salarié mais elle est imputable à l'employeur qui devra en supporter les conséquences financières.

Cependant, la reconnaissance de droits exorbitants, qui ne sont accordés qu'aux seuls salariés protégés, implique corrélativement, au sens de l'analyse juridique, des devoirs. C'est cette interdépendance droits / devoirs qui fonde l'idée de statut. C'est pourquoi le comportement des délégués grévistes se doit, selon la jurisprudences d'être "exemplaire" et se trouve, de ce fait, apprécié plus sévèrement que celui des autres salariés : "si les délégués du personnel ont pour mission de présenter aux employeurs les revendications individuelles ou collectives qui n'ont pas été directement satisfaites, ils ne peuvent le faire dans des conditions d'agitation, de désordre et de violence que l'exercice normal de leurs fonctions aurait permis d'éviter' ${ }^{2}$.

Il en découle un régime juridique particulier qu'illustre assez bien la jurisprudence du Conseil d'Etat sur la demande de licenciement pour faute grave: "dans le cas où la demande est motivée par un comportement fautif, il appartient à l'autorité compétente de rechercher, sous le contrôle du juge de l'excès de pouvoir, si les faits reprochés au salarié sont d'une gravité suffisante pour justifier son licenciement, compte tenu de l'ensemble des règles applicables au contrat de travail de l'intéressé et des exigences propres à l'exécution normale du mandat dont il est investi" ${ }^{3}$. Une telle interprétation se justifie par le fait que le salarié protégé est sous contrat et sous statut, qu'il se situe dans un lien de subordination en même temps qu'il dispose de ce que la Chambre criminelle de la Cour de cassation identifie comme des "prérogatives statutaires" ${ }^{4}$. C'est ce qui explique que la Cour de cassation a pu décider, dans un arrêt déjà ancien mais toujours d'actualité, que la suspension du contrat de travail ne faisait pas obstacle au maintien des missions des représentants du personnel ${ }^{5}$. C'est cette même logique qui vise à assurer la continuité du mandat qui justifie la réintégration du salarié protégé licencié sans autorisation administrative. L'employeur commet, le cas échéant, un délit d'entrave. En conséquence, même en cas de mise à pied disciplinaire, "le représentant du personnel sanctionné est habilité à exercer son mandat sur le lieu même où il lui est interdit d'accomplir le travail" "C'est bien l'appartenance à la collectivité du travail qui fonde, avant tout, la poursuite du mandat en dépit des obstacles qui pourraient l'entraver" ${ }^{8}$. Il en résulte que ce régime exorbitant se révèle particulièrement contraignant pour l'employeur : il lui impose le maintien du salarié protégé dans l'entreprise dans le but de garantir la continuité du mandat et porte, de

\footnotetext{
${ }^{1}$ Cour de Cassation, Chambre sociale, 16 mars 2005, Carcoop France, PBRI, D. 2005, p. 1613, note J. Mouly ; Semaine soc. Lamy 29 mars 2005, n 1208, p. 13, obs. F. Duquesne. Lire également, l'article de J. Mouly, " 'Coup de vent' sur le statut des salariés protégés, Dr. Soc. sept.-oct. 2005, p. 861 à 865 ; Ph. Waquet, «Coup de vent sur la jurisprudence Perrier », RJS 6/05, p. 419 à 422.

${ }^{2}$ Cass. soc. 27 nov. 1968 , Juris. Soc. $\mathrm{n}^{\circ} 279$, p. 30

${ }^{3}$ CE 11 oct. 1995, Simepa, non publié, $N^{\circ} 121772$.

${ }^{4}$ Cass. Crim. 11 juin 1974, Baudry.

${ }^{5}$ Cass. Soc. 23 oct. 1958, Bull. $\mathrm{n}^{\circ} 1068$.

${ }^{6}$ F. Duquesne, op. cit., p. 604.

${ }^{7}$ Voir les arrêts Perrier, Cass. Chambre mixte 21 juin 1974, Perrier, Dalloz 1974, p. 593, Concl. Touffait, p. 596 ; Cass. Soc. 18 nov. 1998, n 498, David c/ Groupe Casino qui concernait les effets de l'annulation d'une décision administrative de licenciement : "dès l'instant que le travail qu'il accomplissait existait toujours, [le salarié] avait droit d'être réintégré dans son emploi, peu important que l'employeur ait entendu supprimer le poste pour occuper lui-même les fonctions de sécurité" ;

${ }^{8}$ F. Duquesne, op. cit., p. 604.
} 
facto, atteinte à son pouvoir de direction ${ }^{1}$ tel qu'il a été forgé par la jurisprudence de la Chambre sociale de la Cour de cassation ${ }^{2}$. Il constitue un cadre juridique strict qui règle précisément les conduites à tenir.

Une telle analyse renvoie incontestablement à une certaine représentation du mandat dont il apparaît nécessaire de questionner la pertinence au travers de cette étude.

\section{II- Les différentes configurations du mandat}

Ce cadre juridique délimite les règles du jeu des rapports entre salariés et employeur. Il impose une «manière de voir» commune. Toutefois, chaque joueur, pour reprendre la terminologie d'Elias, peut toujours choisir, ou jouer involontairement, des coups "hors des règles". En outre ce cadre juridique est une norme de contenant, et non de contenu. Elle laisse une grande marge d'interprétation aux joueurs. Ceci explique le constat d'une pluralité de comportements ou pour être plus précis, une pluralité de configurations relationnelles, au sens que leur donne Elias.

Pour comprendre les différentes configurations que peuvent emprunter les relations entre salariés protégés et entreprise qui peuvent conduire in fine au licenciement, il faut tout d'abord distinguer deux manières d'occuper le mandat fortement opposées :

- Pour certains salariés protégés les intérêts des salariés et les intérêts de l'entreprise (ou plutôt du patronat dans ce cas-là) sont perçus comme totalement antagonistes ;

- Pour d'autres salariés protégés au contraire, les intérêts des salariés ne sont pas obligatoirement opposés à ceux de l'entreprise et parfois même leurs intérêts propres leur paraissent totalement intégrés à ceux de l'entreprise.

On peut ainsi se représenter les différentes manières qu'ont les individus de concevoir le mandat de manière graphique, le long d'une droite dont ces deux manières constituent les deux extrémités. Les comportements individuels sont influencés par la plus ou grande proximité des systèmes de préférence individuels avec ces deux pôles : ils sont plus ou moins influencés par l'un des deux sans que l'on puisse résumer la totalité de leurs actions uniquement par l'opposition ou uniquement par l'adhésion à l'entreprise. Personne ne croit que son intérêt est totalement opposé aux intérêts de l'entreprise, comme personne ne croit qu'il puisse y avoir confusion totale. De la même façon, les salariés qui voient des différences

\footnotetext{
${ }^{1}$ Suivant un récent revirement de jurisprudence unifiant les deux régimes juridiques, la mise à pied d'un représentant du personnel, qu'elle soit conservatoire ou disciplinaire, n'a pas pour effet de suspendre l'exécution de son mandat (seule la mise à pied disciplinaire n'affectait plus, depuis un arrêt de 1999, le mandat qui ne se trouvait plus suspendu durant l'exécution de la mesure (Cass. Soc. 2 mars 2004, Agence Littoral de la société Alsthom Contracting Nord et Est, $n^{\circ} 464$ FS$\mathrm{P}+\mathrm{B}+\mathrm{R}+\mathrm{I})$.

C'est aussi ce qui explique que la mise à pied conservatoire prononcée en vue d'un licenciement impose au représentant du salarié de cesser ses fonctions, tant à l'intérieur qu'à l'extérieur de l'entreprise ; il ne peut ainsi prétendre au paiement des heures de délégation : Cass. Crim. 5 mars 2002, RJS 6/02, $\mathrm{n}^{\circ} 695$

${ }^{2}$ La notion de "pouvoir de direction" fonde en même temps qu'elle légitime certaines décisions prises par l'employeur (en matière, par exemple, de modification des conditions de travail des salariés) ; pour une illustration jurisprudentielle, cf. Cass. Soc. 30 avril 2003, publié au bulletin.; ou encore Cass. Soc. $1^{\text {er }}$ avril 2003

L'employeur peut toujours, le cas échéant, apporter la preuve de l'existence d'un détournement des fonctions de représentation : il y a alors fraude à la loi (le salarié protégé a, par exemple, cherché à se faire élire ou nommer représentant du personnel ou DS pour échapper à un licenciement (Soc. 18 nov. 1999, Dr. Soc. 2000, p. 190, cf. J.-M. Verdier, "Désignation d'un délégué syndical : charge de la preuve et définition de la faute"). Encore faut-il qu'il parvienne à prouver que le but exclusif de la désignation était de faire échec au pouvoir unilatéral de l'employeur, l'existence d'une activité syndicale extérieure écartant par ailleurs cette exclusivité (Cass. Soc. 18 nov. 1999).
} 
fortes entre leurs intérêts et ceux de l'entreprise employeuse souhaitent pourtant y être un minimum intégrés. La différence est donc de niveau et non de nature.

Les résultats présentés ici doivent donc être compris comme des idéaux-types qui ne se rencontrent jamais à l'état pur dans la réalité. Les différences de populations ou de comportements que nous présentons ne sont pas des typologies au sens où on l'entend habituellement, c'est-à-dire la description et le classement d'une population en souspopulations à partir d'une déclinaison d'une ou plusieurs variables appliquées de manière homogène. Ces catégories issues de l'analyse sont beaucoup plus des catégories idéalestypiques dans le sens où des phénomènes concernant tout agent ou toute partie de l'organisation sont particulièrement mis en lumière pour décrire un groupe d'agents ou un phénomène organisationnel. Elles sont une simplification qui permet de mettre crûment en lumière des phénomènes qui sont, dans la réalité, beaucoup plus discrets et moins généraux que ce qu'on pourrait penser d'après la présentation que nous en faisons. Ce modèle d'analyse, basé sur la méthodologie des idéaux-types formalisée par Weber (1971), présente l'énorme avantage de rendre très visibles les phénomènes qui se déroulent dans une organisation. En revanche, et c'est le prix à payer pour ce surplus de visibilité, ils apparaissent comme des caricatures d'une réalité dont les nuances ne sont pas prises en compte. L'opposition salarié entreprise et l'intégration représentent deux "idéaux types".

\section{A) La différenciation des positions ou l'opposition des intérêts}

La première manière d'occuper le mandat, l'opposition, renvoie à des modalités bien connues car leurs fondements ont été formalisés et défendus dans leur expression la plus extrême par tous les courants révolutionnaires et marxistes. Mais toutes les organisations syndicales ont été créées sur ces mêmes fondements sous des formes plus ou moins euphémisées.

Élément fondateur du syndicalisme, ce principe d'opposition d'intérêts, de conflit des logiques, marque très fortement les orientations des organisations syndicales et de leurs militants. Dans tous les stages de formation organisés par l'Institut des sciences sociales du travail à destination des trois organisations syndicales, CGT, CGT-FO et CFDT, cette opposition de nature est défendue par quasiment tous les militants syndicaux.

Par contre, dans l'enquête, cette orientation est loin d'être majoritaire chez les salariés protégés rencontrés. Elle concerne surtout les plus "investis" dans le militantisme et se retrouve chez ceux qui occupent les mandats les plus politiques, notamment celui de délégué syndical. Pour les autres, une telle orientation n'apparaît que résiduelle, intégrée à des positions plus consensuelles, même si elle peut brutalement reprendre le dessus dans les phases de cristallisation des conflits.

En outre, si cette orientation oppositionnelle structure la manière dont certains salariés protégés conçoivent leurs relations à l'entreprise, elle n'apparaît pas toujours de manière marquée dans les entretiens.

1) La référence à l'intérêt collectif 
La référence à l'intérêt collectif peut comporter deux facettes : il peut s'agit de la référence à l'intérêt de l'ensemble des salarié (1) ou de la référence au groupe professionnel (2).

\section{La référence à l'intérêt de l'ensemble des salariés}

Pour ces salariés protégés, l'opposition entre salariés et patronat est une opposition de nature : les salariés et les dirigeants d'entreprise occupent dans le champ social des positions organiquement différentes, ce qui a pour effet d'inscrire l'antagonisme comme mode normal des relations collectives dans l'entreprise. Cette opposition est entretenue par l'impression, fondée ou non, que les dirigeants ne tiennent pas compte de l'avis des salariés.

\footnotetext{
"Je pense qu'il considère le personnel avec mépris. Il n’y avait aucune écoute, aucune réponse à des interrogations et puis derrière, il y avait tout un tas de manipulations." (Salarié protégé, délégué syndical, membre CHSCT, syndiqué, ouvrier, 44 ans, CAP, demande de licenciement pour faute)
}

On a ainsi une opposition forte entre les dirigeants et ceux qui leur font allégeance et les salariés "lambda", qui font l'objet de traitements différenciés.

"Il ne gardait que les cadres à Nanterre, il n'y a qu'eux qui travaillaient, c'était des copains, des copines.» (Salariée protégée, juge prud'hommes, syndiquée, 54 ans, agent de maîtrise, CAP, PME, demande de licenciement pour inaptitude)

Pour ces salariés, c'est principalement leur force de travail qui, vendue à l'employeur, est mobilisée dans l'entreprise. Ils accordent alors une place importante aux obligations issues de la relation de travail qui, de leur point de vue, ne peut exiger une obéissance trop étendue.

"Je lui ai immédiatement répondu, moi et vous n'avons rien en commun, je travaille pour S., mais pas pour vous, je ne deviendrai jamais quiconque pour vous, je fais mon boulot." (Secrétaire du comité d'entreprise, PME)

La négociation salariés - entreprise, sur le principe d'intérêts divergents, vise à optimiser les termes de l'échange : réduire la force de travail mobilisée ou accroître les contreparties (principalement financières). Compte tenu de l'inégalité entre salariés et patronat, la négociation, comme ce qui en résulte, doivent être référés au collectif qui seul permet de réduire un peu l'inégalité de départ et d'imposer une modification de l'échange.

"Avec mes autres collègues, pendant un an et demi de crise, on s'est serré les coudes." (Salarié protégé, délégué syndical, membre CHSCT, syndiqué, ouvrier, 44 ans, CAP, demande de licenciement pour faute)

Le collectif est en effet une ressource systématiquement recherchée en cas de menaces ou de rétorsions de la part des employeurs; ceci pour deux raisons : parce qu'elle est une ressource efficace, mais aussi parce que ce n'est pas l'individu qui est attaqué mais le représentant du collectif. En ce sens, le soutien collectif traduit bien le fait que le salarié protégé occupe un mandat de représentant et qu'il n'a pas été désigné à titre personnel.

\footnotetext{
"Oui j'ai eu beaucoup de mal (à avoir des témoignages), parce que quand on est dans des situations comme ça aussi délicates, les amis se barrent en courant. J'ai eu la chance d'avoir des collègues qui avaient confiance en moi et avec qui j'ai eu de bons rapports. Ils ont eu le courage effectivement de témoigner. [...] J'avais 3 témoignages à l'époque, sur 70 personnes, ce n'était pas énorme, mais ça suffisait, c'était des gens qui prenaient des risques, un peu plus que ceux (qui
} 
témoignaient pour la direction) qui eux ne risquaient rien puisqu'ils allaient dans le sens de la direction. Donc c'est vrai que quand on a monté le dossier pour les prud'hommes avec les collègues de la CFDT, c'était important d'avoir ces témoignages.» (Salarié protégé, délégué syndical, membre CHSCT, syndiqué, ouvrier, 44 ans, CAP, demande de licenciement pour faute)

Dans cette logique, l'adhésion à une organisation syndicale va presque de soi. C'est en effet le collectif qui sert de référence mais qui oriente les revendications portées par les salariés protégés.

"J'ai rétabli un peu une certaine discipline, une certaine situation, qui a fait qu'il y a quand même eu certains bénéfices pour les salariés : remise à jour des conventions collectives, remise à jour de leur application, c'est vrai qu'il y avait un laisser-aller de ce côté. [...] Non, la convention collective n'était pas respectée, d'autant plus que les conditions de travail au point de vue vêtements, chaussures, rien n'était respecté. Pendant 2 ans, j'ai lutté là-dessus, j'ai réussi à instaurer ce système.» (Salarié, juge prud'homal, délégué syndical, ouvrier, 58 ans, CEP, TPE, demande de licenciement pour inaptitude)

Cette référence au collectif s'assied bien sûr sur un rejet de tous les comportements uniquement guidés par l'intérêt individuel.

"On n'était pas vraiment copain tous les deux. [...] C'est une personne qui est venue au syndicalisme très tard. Là je porte un jugement, mais il l'a fait plus par intérêt." (Salarié protégé, délégué syndical, membre CHSCT, syndiqué, ouvrier, 44 ans, CAP, demande de licenciement pour faute)

Les avis sur la possibilité de signer une transaction sont, dans ce cas, assez tranchés, comme nous aurons l'occasion de le voir plus loin.

"Ça peut être (une magouille) si ce n'est pas transparent. [...] Parce qu'il y a des gens qui pourraient profiter de leur mandat pour se vendre.» (Salarié protégé, délégué syndical, membre CHSCT, syndiqué, ouvrier, 44 ans, CAP, demande de licenciement pour faute)

Pour ces salariés, la place importante occupée par le mandat, la référence au collectif, c'est-àdire ici l'ensemble des salariés, tout comme les références morales auxquels ils se réfèrent, donnent l'impression d'une grande cohérence avec les conceptions juridiques qui lient l'exercice du mandat et l'existence d'un statut spécifique, que nous avons analysé précédemment.

\section{La référence au groupe professionnel}

Toutefois, l'occupation du mandat varie, l'éloignant parfois de l'approche statutaire qui est celle retenue par le Droit. En effet, les logiques oppositionnelles ne sont toutes pas construites en référence à la totalité des salariés. Parfois, il s'agit de la référence à un groupe plus étroit avec lequel on partage la même perception quant à la manière dont l'entreprise traite les salariés.

C'est par exemple le cas du "couple" de salariés dont les citations sont relativement congruentes et qui vont ensemble décider de se présenter aux élections pour tenter de modifier les choses.

"Je me suis quand même rendu compte que très rapidement que, quoi je fasse, j'étais toujours en dessous de l'objectif. [...] Donc, au bout d'un an et demi, que vous travaillez et que vous ne voyez 
rien venir, vous vous posez quand même des questions.» (Salarié protégé, membre du comité d'entreprise, délégué du personnel, syndiqué, 37 ans, cadre, Bac+2, PME, demande de licenciement pour faute)

"On s'est rendu compte qu'on a amené notre savoir, nos capacités, et à côté de ça on n'était pas rémunérés en conséquence et que la société travaillait à l'ancienne. Donc il y avait un gros décalage contrairement à d'autres boîtes où on arrive, il y a une formation, on a chez eux en sortant une expérience enrichissante. Or là, on s'appauvrissait, on donnait notre expérience et on n'avait rien en retour.» (Salarié protégé, délégué syndical, cadre, 42 ans, Bac, PME, demande de licenciement pour faute)

Ils arrivent dans un premier temps à fédérer les mécontentements pour constituer une liste, mais comme ils l'indiquent clairement, il ne s'agit pas de construire un collectif de références mais de se doter d'une ressource collective qui puisse être utilisée lors de l'affrontement dont le fondement est individuel.

\begin{abstract}
"Du coup ils ont été coincés, ils n'ont pas eu le temps de syndiquer les gens pour le patron, ils ne nous ont pas vu venir sur ce coup-là. Donc on se fait élire et l'on arrive au comité d'entreprise avec les pleins pouvoirs parce qu'on avait mis que des petits copains, on avait monté des listes à nous en faisant syndiquer d'autres personnes.» (Salarié protégé, membre du comité d'entreprise, délégué du personnel, syndiqué, 37 ans, cadre, Bac+2, PME, demande de licenciement pour faute)

"Y., qui avait les mêmes soucis que moi, et on commençait à en avoir un peu ras-le-bol et à ne pas être entendus, à être pris pour des imbéciles à chaque fois qu'on disait quelque chose, on nous disait qu'on avait tort. Et surtout, on avait aucune information, ni sur la société, ni sur les résultats de notre magasin, ni sur rien. [...] On essaie de faire bouger les choses. On n'a pas 50 moyens de faire bouger les choses, on s'est dit la seule manière, en plus ça tombait bien à ce moment-là parce qu'il y allait avoir des élections du personnel, on s'est dit, on va essayer de se faire élire.» (Salarié protégé, membre du comité d'entreprise, délégué du personnel, syndiqué, 37 ans, cadre, Bac+2, PME, demande de licenciement pour faute)
\end{abstract}

\title{
L'alliance peut, dans ce cas, vite devenir fusionnelle.
}

"On est de très bons amis avec Y., c'est qu'on était très complémentaires, dans tout ce qu'on faisait. [...] Y me manque beaucoup dans la société, parce que je n'arrive pas à me battre tout seul. [...] Donc on avait des visions d'entreprise qui étaient un peu différentes et ça nous a permis de beaucoup nous compléter et on avait la même soif de faire changer les choses, et on s'est rapidement rendu compte que sur beaucoup de choses, on avait exactement la même vision sur la société, sur l'évolution de la société. On s'est vraiment trouvé, on rencontre peu de personnes comme ça dans sa vie, c'est un alter ego, c'est quelqu'un de complémentaire avec qui j'ai des affinités extraordinaires." (Salarié protégé, membre du comité d'entreprise, délégué du personnel, syndiqué, 37 ans, cadre, Bac+2, PME, demande de licenciement pour faute)

Mais on imagine bien que, dans ce cas, le soutien d'un collectif plus large ne va pas de soi, car ce groupe fondé de manière conjoncturelle apparaît très hiérarchisé, ce qui ne facilite pas le sentiment d'appartenance au même collectif pour les salariés qui sont, de fait, exclus de cette hiérarchisation.

"(L'activité au niveau du comité d'entreprise était plus intéressante ?) Il y avait un peu de ça, oui, on s'est pris au jeu, on a trouvé le truc sympa. [...] On a donc fait venir quelqu'un d'autre (pour être secrétaire du comité d'entreprise) mais c'est nous qui dirigions les trucs, les gens étaient là pour occuper les places et de temps en temps participer au débat, mais il faut être clair, c'est nous qui dirigions. Et à 2 pendant 2 ans, on a tout fait tourner.» (Salarié protégé, membre du comité d'entreprise, délégué du personnel, syndiqué, 37 ans, cadre, Bac+2, PME, demande de licenciement pour faute) 
Ceci explique que certains salariés, de leur propre chef ou / et poussés par les dirigeants, puissent vouloir installer un jeu plus ouvert.

"Je me suis présenté afin qu'on puisse renouveler le dialogue social entre la direction et l'ensemble
des salariés de l'entreprise puisqu'il n'y avait plus de dialogue possible depuis plus d'un an entre
les représentations syndicales et la direction. Donc aucune avancée, aucun progrès social pour les
salariés. [...] On subit un lourd héritage de nos prédécesseurs puisque la direction a eu un peu de
mal à entamer un dialogue.» (Secrétaire CE, non-syndiqué, PME)

Les oppositions de certains salariés à ce fonctionnement peuvent être encore plus marquées, au point que les nouveaux élus votent pour le licenciement de leurs prédécesseurs.

"Donc il y a eu une consultation pour un avis du comité d'entreprise en début d'année concernant le licenciement de x. mais le comité d'entreprise, à l'époque a donné un avis favorable.» (Secrétaire CE, non-syndiqué, PME)

\section{2) L'idéologisation du mandat ou le primat des principes}

On pourrait avoir l'impression jusqu'ici que l'opposition d'intérêts entre les salariés et l'entreprise est le produit d'une volonté personnelle qui ne doit pas grand-chose à l'histoire ni au contexte social et professionnel. Ce n'est bien sûr pas le cas. En effet, les caractéristiques individuelles, notamment l'histoire personnelle et sociale des individus, peuvent faciliter ce type de logique. D'autre part, ce positionnement est plus ou moins facile suivant le type d'entreprise où le salarié protégé est employé.

Cette différenciation des positions est très souvent assise sur une histoire personnelle singulière dans laquelle le sentiment d'injustice apparaît de manière importante. L'enquête n'était pas centrée sur la construction de la sensibilité au militantisme, nous nous référons ici à des travaux antérieurs dans lesquels cet outillage théorique a été construit (Correia, 1995).

Il nous faut simplement préciser brièvement ce que l'on entend par "sentiment d'injustice". Tout d'abord il s'agit bien d'un sentiment et non de situations d'injustice ${ }^{1}$. Le jugement porté par l'individu est alors central et des individus différents peuvent, dans une même situation, porter des appréciations différentes.

Le sentiment d'injustice est articulé à un système de positions sociales. L'individu qui ressent un sentiment d'injustice trouve que la position occupée ne correspond pas à la valeur réelle de celui qui l'occupe. Ou plutôt il trouve que le traitement appliqué à ces positions n'est pas juste par rapport à la valeur réelle des individus qui les occupent. Nous appellerons ce type de sentiment "injustice de classe". Ce sentiment peut être créé par des contraintes générales qui ont obligé l'individu à s'engager dans des voies qu'il ne souhaitait pas. Cette injustice est parfois généralisée à l'ensemble des proches. Dans ce cas, l'injustice (injustice de classe) peut être considérée comme un capital partiellement transmissible, qui rend l'engagement dans le militantisme "naturel". Il s'agit en grande partie de payer une dette envers ceux qui vous ont élevé.

\footnotetext{
1 À partir du moment où nous parlons de sentiment d'injustice, il est clair que la situation déclenchante ne peut être caractérisée de juste ou d'injuste, c'est la perception de l'individu qui est importante. Dans la suite du texte, c'est dans ce sens que nous utiliserons à chaque fois le terme injustice et ses dérivés.
} 
Bien sûr si on parle d'injustice de classe ou si on parle de sentiment d'être mal traité, l'on suppose que ce sentiment a d'autant plus de chance de se concrétiser pour celui qui occupe un emploi moins qualifié et/ou qui dispose d'un niveau de formation peu élevé.

Cette sensibilité particulière à l'injustice porte tout naturellement les militants à devenir des militants dans les lieux les plus variés : mouvement politique, associations locales, mouvements confessionnels, groupe de théâtre... tous les lieux où l'on peut impulser du changement social, corriger d'une manière ou d'une autre le traitement appliqué aux catégories dont ils sont originaires. Cet engagement aux multiples facettes est toujours construit autour de finalités voisines qui donnent une cohérence d'ensemble au comportement des individus. C'est cette sensibilité à l'injustice qui explique l'investissement dans le mandat car il apparaît comme une manière pratique d'intervenir pour changer l'ordre des choses.

"Parce qu'il y avait des problèmes assez injustes dans l'entreprise, il se passait des choses injustes
dans les salaires, il fallait mettre le nez dedans et personne n'osait. Mais de fil en aiguille, quand ça
m'a atteint personnellement, je me suis dit : il faut faire quelque chose, ça ne peut plus durer.»
(Salariée protégée, juge prud'hommes, syndiquée, 54 ans, agent de maîtrise, CAP, PME, demande
de licenciement pour inaptitude)

"Quand je suis arrivé dans cette boîte, [...] je sentais qu'il y avait quand même un malaise, un besoin de s'exprimer et donc on en a discuté avec les collègues en se demandant s'il n'y avait pas besoin d'un DP. [...] Et là j'ai dit, je suis partant et c'est comme ça que l'aventure a commencé donc je me suis fait élire DP." (Salarié protégé, délégué syndical, membre CHSCT, syndiqué, ouvrier, 44 ans, CAP, demande de licenciement pour faute)

"Je ne l'ai jamais été (syndicaliste), je n'avais pas de raisons, mais après il y a eu des évènements qui ont fait qu'il fallait bien qu'on se défende." (Secrétaire du comité d'entreprise, déléguée syndical, 58 ans, PME)

De manière évidente, ce sont les salariés les plus "politisés qui partagent ce point de vue. L'occupation d'un mandat s'inscrit ainsi dans un terrain idéologiquement fertile, car ils ont toujours été sensibles aux inégalités sociales ; c'est ce qui les conduit d'ailleurs à vouloir les corriger.

\footnotetext{
"J'ai toujours eu un certain esprit syndicaliste, ou un certain sens de la justice aussi." (Salarié, juge prud'homal, délégué syndical, ouvrier, 58 ans, CEP, TPE, demande de licenciement pour inaptitude)
}

Toutefois on ne peut réduire les logiques oppositionnelles à des effets de l'injustice de condition. Une forme plus faible de ressenti de l'injustice peut aussi conduire à une logique oppositionnelle.

En effet, la source du sentiment d'injustice n'est pas exclusivement liée au milieu d'origine. Ce sentiment peut aussi être suscité par un événement qui survient dans la vie professionnelle, qu'il touche l'individu, un de ses proches ou le groupe auquel il appartient ou se réfère. Il en est ainsi quand l'individu trouve que le groupe auquel il fait partie n'est pas traité de manière correcte; c'est-à-dire que le traitement qu'on lui applique ne correspond à sa valeur "réelle". Ceci est particulièrement frappant dans les professions de la santé où la référence à une profession qui n'est "pas assez considérée" est omniprésente. Il s'agit bien sûr des professions dominées du champ professionnel et le sentiment d'injustice est dirigé vers les professions dominantes du même champ. Nous appellerons ce type de sentiment "injustice de profession". 
C'est à cette sorte d'injustice que s'accolent, par exemple, les individus que nous avons décrits plus haut dans la référence au groupe professionnel.

\section{3) Les effets des candidatures collectives}

Le fait syndical, l'accès à des fonctions représentatives ont alors pour effet, qui s'explique en partie par les raisons pour lesquels les individus postulent pour les mandats, d'équilibrer la relation entre eux et les dirigeants de l'entreprise.

Dans ses formes les moins conflictuelles, l'entreprise ou ses dirigeants considèrent de leur côté que la représentation, loin d'être une simple obligation ou une gêne, constitue une opportunité pour l'organisation qui bénéficie ainsi d'un interlocuteur nécessaire à la gestion des hommes. (cf. citations précédentes). Compte tenu de cet a priori favorable, les salariés protégés bénéficient ainsi d'une augmentation de leur valeur sociale.

Mais même dans une situation où les ressources entre interlocuteurs sont déséquilibrées, les interlocuteurs peuvent se satisfaire de cette situation d'inégalité des ressources et installer un système d'égalité relative des positions en se partageant les rôles : par exemple les représentants des salariés se font les portes parole des situations de terrain tandis que la hiérarchie se positionne sur le versant comptable et économique. Sous réserve d'une acceptation réciproque, on peut avoir entre interlocuteurs des relations de bonne entente qui se trouvent en quelque sorte pacifiées.

Toutefois, la valorisation sociale dont bénéficient ces salariés ne peut que difficilement se traduire en promotion professionnelle. En effet, les mandats, faisant référence à un collectif, imposent aux salariés des devoirs moraux. Les avantages dont ils disposent constituent, en quelque sorte, le prix à payer pour ces contraintes ${ }^{1}$.

Une fois élu, ses mandants attendent souvent du salarié protégé qu'il soit exemplaire. Cette exemplarité impose des contraintes extrêmement fortes, car elle induit une norme de comportement impossible à atteindre. Ce décalage entre une représentation idéale à laquelle doit se conformer le mandataire et ses propres limites est créatrice d'une certaine fragilité. Parce qu'elle implique une responsabilité du mandant, cette fragilité se répercute sur le salarié protégé, qui ne peut pas se cantonner à apprécier ses actes ou ses prises de position en fonction de leur justesse, mais est obligé de pondérer sa position en prenant en compte les effets que ses prises de position peuvent générer. On est là dans l'opposition classique que fait Weber (1959) entre une éthique de conviction et une éthique de la responsabilité.

\footnotetext{
"J'ai commencé un boulot que je n'ai pas fini, les collègues qui sont restés sont maintenant massacrés par la direction, et d'autre part j'étais délégué et j'ai été viré suite à la grève mais moi dans ma tête, je suis encore chez S." (Salarié protégé²)
}

\footnotetext{
${ }^{1}$ Sans référence ici à la manière dont le législateur a pensé cette protection. Il s'agit plus de la manière dont les salariés protégés (du moins ceux qui pensent représenter le collectif) conçoivent cette protection.

${ }^{2}$ Cette citation et les 4 qui suivent sont tirées d'entretiens menés dans la phase de pré-enquête auprès de salariés d'une même entreprise; où un mouvement social extrêmement dur, s'était traduit par le licenciement d'une partie des grévistes. C'est en partie ce paroxysme dans le conflit qui donne à voir aussi nettement les oppositions de principe, ou cette éthique de conviction si on adopte les termes de Weber. Sont interrogés en même temps des représentants du personnel qui ont été licenciés et des salariés qui sont encore en poste dans l'entreprise. Compte tenu de cette configuration particulière et parce que le conflit est loin d'être clos, nous ne donnons ici aucune indication permettant de reconnaitre les individus interrogés.
} 
Cette éthique de la responsabilité interdit, par exemple, que les salariés protégés soient "les premiers servis", en cas de règlement avantageux du conflit. Elle introduit, en fait, un biais en défaveur des salariés protégés

"Les gens qui étaient licenciés ne sont pas partis avec les poches vides, sauf nous. [...] Les gars qui étaient licenciés, les salariés, ils sont partis avec plus que leur salaire. C'est nous qui avions décidé, puisqu'on avait fait une commission, parce qu'on était organisés à l'intérieur de la grève, on avait fait 2 berges d'autoroutes, [...] On a rapporté des sous, on ne savait pas que ça rapportait tant ! On a même pensé se recycler là-dedans, mais entre les 2 péages, honnêtement, on a fait 10 millions, à peu prés. (On a décidé de payer plutôt), pour ceux qui étaient licenciés." (Salarié protégé)

La protection dont ils disposent peut même apparaître, à leurs propres yeux, non méritée.

"Moi je vais vous dire honnêtement, je regrette un peu de n'avoir pas été licencié, parce que ça me fait culpabiliser. On me dit le Délégué Syndical n'a pas été licencié, ça m'a fait chier parce que tous les pauvres innocents ont été licenciés. Moi honnêtement, j'aurais voulu être licencié, clair et net." (Salarié protégé)

De manière plus générale, tout avantage ou mesure perçue comme telle par les autres salariés leur est quasi systématiquement reproché.

"Sérieusement, les gens sont individualistes, c'est une horreur, pour les motiver, il faut vraiment qu'il y ait une catastrophe, et je vous assure quand on est parti, pour en revenir à S. on avait l'impression qu'on était des bonnes sœurs, les gens se sont sentis trahis. [...] C'était très dur, on nous a vraiment reprochées de partir, c'étaient comme si on les abandonnait." (Salarié protégé)

De ce fait, la norme morale qui s'impose au salarié protégé est d'autant plus forte que, quelle que soit la nature du conflit, le type de relations entre salarié et salarié protégé ou le degré d'exemplarité des comportements des salariés protégés, ceux-ci sont souvent "soupçonnés" de bénéficier d'un traitement particulier. On retrouve alors en filigrane l'idée que les salariés protégés ne payent pas réellement les conséquences des comportements collectifs.

"Les salariés disaient ouais mais vous, vous êtes protégés." (Salarié protégé)

\section{L'idéologisation des relations et l'objectivation des oppositions}

La référence au collectif, associée au fait que la différenciation salariés/entreprise est considérée comme quasi organique, conduisent à ce que les désaccords avec l'entreprise tendent à devenir des désaccords de principe : tout ce qui vient de l'entreprise doit ainsi être rejeté.

"On n'était pas du tout favorable sur le plan de licenciement, sur tous les plans d'ailleurs on a toujours dit non. Et on a toujours dit que la direction se fichait de nous." (Secrétaire du comité d'entreprise, déléguée syndical, 58 ans, PME)

Le salarié évoque la tentative de l'entreprise de l'attirer de son côté pour mieux souligner le caractère inconvenant voire hors de propos de cette proposition.

"(Le directeur) a essayé de m'acheter : il m'a proposé de devenir Chef avec une augmentation de 400 Francs par mois, il me propose donc de devenir un petit chef." (Salarié protégé) 
Cette objectivation a pour effet de donner une certaine stabilité aux relations tout en les dotant d'une certaine inertie. Les salariés protégés sont ici mieux protégés des "à-coups", et donc, des licenciements. Surtout, les désaccords professionnels sont minorés par ces effets d'opposition systématique. Ils sont cantonnés au statut du salarié protégé et ne touchent pas la personnalité de celui-ci et n'ont que peu d'impacts sur les autres aspects de sa vie.

\begin{abstract}
"J'étais bien dans ce que je faisais et je trouvais que je m'y épanouissais bien, je trouvais un équilibre entre mes activités professionnelles et extra-professionnelles. J'étais tellement investi dans le monde associatif que tous les petits déboires, toutes les petites brimades que je pouvais subir dans ma vie professionnelle, une fois sorti je n'y pensais plus, je me replongeais dans la vie associative.» (Salarié protégé, délégué syndical, membre CHSCT, syndiqué, ouvrier, 44 ans, CAP, demande de licenciement pour faute)
\end{abstract}

Dès lors que les affrontements sont des affrontements de position, la volonté individuelle, mais aussi son investissement affectif, se trouvent faiblement mobilisés. On peut, par exemple, être très étonné de la violence des interpellations que certains syndicalistes adressent à leur direction et qui sont suivis de rapports apparemment cordiaux dès la porte de la salle de réunion franchie. L'explication tient à la séparation assez rigide de la position de l'individu et de sa personnalité comme si, pour ces salariés protégés, on pouvait jouer des deux registres à des moments différents sans que cela prête à conséquence.

Dans une telle configuration, le licenciement apparaît comme une anomalie, constat que nous développerons plus loin.

\title{
La création d'un espace de jeu
}

Dans ce mode d'occupation du mandat, le désaccord se gère dans le long terme. Il s'agit d'une configuration oppositionnelle qui peut se lire comme un jeu comportant des règles particulières. Bien sûr, les acteurs ont rarement l'impression de jouer ou de s'amuser, et ce, d'autant plus que les conséquences sont risquées. Leurs conduites peuvent pourtant être analysées comme étant inscrites dans le cadre d'un jeu, d'une configuration relationnelle pour utiliser le vocabulaire d'Elias.

Tout d'abord, les coups que jouent les protagonistes semblent être la conséquence directe des actions de leurs adversaires.

\footnotetext{
"On a remué un peu la merde parce qu'on s'est rendu compte qu'il y avait des choses qui n'étaient pas nettes [...] Par exemple, [...] l'argent du comité d'entreprise n'a jamais été versé, donc cet argent-là, on a essayé de le récupérer. [...] On a fait intervenir l'inspection du travail, on a même fait condamner la société pour délit d'entrave, enfin on avait mis 3 délits d'entraves et il y en a un qui a été accepté. [...] On a pas mal remué dans les brancards, on a réussi quand même à avoir pas mal de choses.» (Salarié protégé, membre du comité d'entreprise, délégué du personnel, syndiqué, 37 ans, cadre, Bac+2, PME, demande de licenciement pour faute)

"Dans le courrier que j'ai reçu, il était indiqué qu'il envisagerait à mon égard des mesures plus dissuasives. [...] Voilà, alors je me suis dit qu'il fallait attaquer. Je suis donc allé aux prud'hommes et j'ai engagé une procédure pour harcèlement et discrimination syndicale.» (Salarié protégé, délégué syndical, membre CHSCT, syndiqué, ouvrier, 44 ans, CAP, demande de licenciement pour faute)
} 
Dans cette logique, le problème n'est pas tant d'avoir des résultats malgré les affirmations des élus, mais de jouer des coups, d'entretenir l'opposition.

\begin{abstract}
"On avait commencé à initier un droit d'alerte parce que la société commençait à aller mal. [...] Ce sont les fameux E-Mail que j'ai envoyés aux investisseurs pour les informer parce qu'on commençait à avoir vraiment la trouille pour la boîte, on sentait vraiment que ça se barrait en couilles, qu'ils ne faisaient rien pour que ça change. Donc on s'est dit qu'avec un droit d'alerte, ils vont être obligés de bouger, parce qu'il va y avoir un rapport, parce qu'on va être obligé d'informer les investisseurs, donc ça va les faire bouger. (Le PDG), dès qu'on va commencer à toucher à son pognon, il va hurler. Le problème, c'est qu'on s'est un peu pris les pieds dans le tapis sur la procédure, pas tellement pour le début, mais c'est vrai qu'on s'est un peu précipité pour les investisseurs parce qu'on était pressés de faire bouger les choses. On a communiqué un peu vite. [...] Je me suis dit, qu'en même temps, on allait leur balancer les tracts qu'on avait fait pour les informer de la vie de la société.» (Salarié protégé, membre du comité d'entreprise, délégué du personnel, syndiqué, 37 ans, cadre, Bac+2, PME, demande de licenciement pour faute)
\end{abstract}

"Ce qui m'intéresse aujourd'hui, c'est de continuer le combat.» (Salarié protégé, membre du comité d'entreprise, délégué du personnel, syndiqué, 37 ans, cadre, Bac+2, PME, demande de licenciement pour faute)

\title{
C'est bien cette perception qu'a l'entreprise dans certains cas.
}

\begin{abstract}
"Ils disent, on est là pour faire du combat, c'est écrit d'ailleurs, les syndicats sont là pour combattre. (Le délégué syndical) m'a dit, moi je me suis syndiqué chez vous parce que j'ai été exploité et (que) je veux me venger. [...]Ils venaient chercher un conflit permanent. [...] Moi j'ai un souvenir caractéristique, 2001, ça a été une bonne année pour nous, on leur avait donné les bilans, mais même les bilans, ils ne les croyaient pas. Ils nous disent : vous gagnez trop. L'année suivante on a gagné moins, c'est sûr. (C'était une opposition) pas constructive. [...] C'est une personne qui à mon sens est une personne aigrie, qui en veut à l'entreprise en tout cas il en veut à tout le monde patronal." (DRH, PME)
\end{abstract}

Cette perception est partagée par le dirigeant qui assiste à l'entretien et qui surenchérit : " (Ils détruisent tout, c'est des destructeurs." (PDG, PME)

Le jeu oppositionnel ne vise pas la destruction de l'adversaire, ce qui conduirait à faire disparaître le jeu. Ce qui est visé, c'est la reconnaissance par l'adversaire de la valeur supérieure à celle qu'on lui accordait en tant que simple salarié.

\footnotetext{
"À partir du moment où l'on a commencé à se lancer là-dedans, très honnêtement, on s'est pris au jeu, c'est-à-dire que très rapidement, on s'est rendu compte des moyens qu'on pouvait avoir, de ce qu'on pouvait faire. [...] On avait un certain pouvoir, (on) pouvait changer les choses. Donc ça, ça nous intéressait beaucoup, donc on s'est franchement pris au jeu et on y a été à fond, comme des malades.» (Salarié protégé, membre du comité d'entreprise, délégué du personnel, syndiqué, 37 ans, cadre, Bac+2, PME, demande de licenciement pour faute)
}

La valeur sociale est ainsi le seul véritable enjeu de l'affrontement. Tout le reste ne représente qu'un moyen de l'obtenir. Les rationalisations argumentaires ne parviennent pas toujours à cacher cette recherche de reconnaissance comme dans la citation ci-dessous (si l'on garde à l'esprit que les bons dont parle le salarié sont son collègue élu et le salarié protégé lui-même).

"Ça nous révoltait complètement parce qu'on cautionnait des gens qui n'étaient pas bons et on virait des gens qui étaient des bons. Tous les bons qui se rendaient compte que ça n'allait pas et qui l'ouvraient un peu se faisaient dégager, donc ça finit par énerver. On se dit que tous les gens pas mal qui ont fait des études se font dégager, et tous les merdeux qui magouillent on les cautionne. C'est aussi pour ça qu'on se battait parce que c'était franchement injuste, et ce genre 
d'injustice, je ne le supporte pas.» (Salarié protégé, membre du comité d'entreprise, délégué du personnel, syndiqué, 37 ans, cadre, Bac+2, PME, demande de licenciement pour faute)

Ce côté "jouissif" de l'affrontement, qui apparaît alors comme un jeu dont l'issue est cependant risquée, transparaît nettement dans les citations ci-dessus, alors même que l'enjeu est la confirmation ou non du refus du licenciement.

\begin{abstract}
"Ça devient une bataille assez rigolote parce qu'eux amènent un dossier comme ça, donc nous, on amène un dossier comme ça. Comme il faut en amener 3, on vient avec un carton...» (Salarié protégé, membre du comité d'entreprise, délégué du personnel, syndiqué, 37 ans, cadre, $\mathrm{Bac}+2$, PME, demande de licenciement pour faute)

"C'était de bonne guerre, je me suis dit, j'attaque donc lui aussi contre attaque.» (Salarié protégé, délégué syndical, membre CHSCT, syndiqué, ouvrier, 44 ans, CAP, demande de licenciement pour faute)

"Je ne voulais pas signer l'ordre du jour (pour le vote du licenciement) donc j'ai eu un huissier qui est venu chez moi pour me faire signer l'ordre du jour, c'était assez folklorique, je trouvais ça assez marrant.» (Salarié protégé, délégué syndical, membre CHSCT, syndiqué, ouvrier, 44 ans, CAP, demande de licenciement pour faute)
\end{abstract}

Parce que le jeu oppositionnel est un jeu permanent, tout peut servir à ré-amorcer le désaccord. Ceci explique que les salariés protégés sont à l'affût de toutes les occasions de jouer de nouveaux coups, de déséquilibrer leur adversaire. Cette orientation n'est ni un accident ni un effet imprévu, elle est clairement voulue par les salariés qui se présentent à l'élection.

"(Il y a des effets sur votre carrière ?) Ça c'est clair, on le savait dès le départ. [...] (L'autre élu) et moi on a toujours été conscient de ça. Les autres, on leur a expliqué, on leur a dit : attention, si vous vous embarquez là-dedans, il faut quand même savoir que ça risque de vous enquiquiner un petit peu. [...] (Salarié protégé, membre du comité d'entreprise, délégué du personnel, syndiqué, 37 ans, cadre, Bac+2, PME, demande de licenciement pour faute)

\title{
4) Le droit : une ressource garantissant les limites des positions
}

Dans cette logique, le droit constitue une ressource structurelle utilisée dans la confrontation des positions.

On s'est formé, on s'est beaucoup documenté, on a bossé comme des malades pendant 2 ans." (Salarié protégé, membre du comité d'entreprise, délégué du personnel, syndiqué, 37 ans, cadre, $\mathrm{Bac}+2$, PME, demande de licenciement pour faute)

L'usage de ressources juridiques ou l'appel à des experts juridiques par des représentants des salariés tend à se banaliser. Il s'intègre naturellement dans l'ensemble des autres ressources, plus traditionnelles, comme le rapport de forces, utilisées pas les salariés protégés "oppositionnels".

"On a nommé un cabinet d'expert, ils nous ont amené leur expérience. Le plan social a commencé en septembre 2002 et la direction l'a annulé en juillet 2003, on s'est dit, on a gagné la $1^{\text {ère }}$ manche. [...]. On a encore nommé le même cabinet expert qui a estimé que rien n'était justifié, qu'il n'y avait aucune difficulté économique, les seules difficultés venaient de la question globale de l'entreprise, c'était des perspectives, c'était sa capacité à se projeter dans l'avenir et à récupérer des parts de marché. On aurait peut-être pu faire durer le plaisir si on avait le personnel avec nous 
mais une fois de plus, on s'est retrouvé qu'avec 2 ou 3 collègues.» (Salarié protégé, délégué syndical, membre CHSCT, syndiqué, ouvrier, 44 ans, CAP, demande de licenciement pour faute)

Mais même dans des configurations où la différenciation des positions domine, des orientations vers un autre type d'usage des ressources juridiques est constatée.

"C'est amorcé ça (que le droit devienne un outil), on a quelques contentieux, on a des avocats qui surgissent à côté des salariés, tout ça ce n'était pas dans la culture. [...] Il y a (en plus) une culture anglo-saxonne assez forte avec une notion de contrat qui est individuelle. Les deux se superposent, moi je sens très bien une juridicisation des relations sociales.» (DRH, grande entreprise)

\section{B) La personnalisation des mandats}

Contrairement aux salariés protégés que l'on a rattachés à l'idéal type oppositionnel, d'autres salariés protégés construisent leurs actions avec le sentiment de participer à une communauté de destin avec l'entreprise et donc avec les dirigeants, d'être liés par les mêmes contraintes et les mêmes intérêts, comme le manifeste par exemple l'utilisation du pronom on ou du possessif "notre", quand il parle de l'organisation.

Cette conjonction des intérêts est due, en partie, au fait que l'activité professionnelle n'est pas uniquement investie pour ses contreparties financières mais parce qu'elle est supposée être porteuse d'opportunités de réalisation personnelle.

\footnotetext{
"J'avais beaucoup de plaisir à faire mon travail, oui. [...] J'ai évolué quand même très rapidement, en fait, quand j'ai commencé, j'étais vraiment à l'échelon le plus bas dans la convention collective, et 4 ans après, j'étais cadre." (Salarié protégé, membre comité d'entreprise, délégué du personnel, non-syndiqué, 29 ans, cadre, Bac+2, PME, demande de licenciement économique)
}

La reconnaissance que manifeste l'entreprise renforce l'idée de cette valorisation de l'individu.

"Le directeur ne tarissait pas d'éloges à mon encontre, donc c'était bien." (Salarié protégé, membre comité d'entreprise, délégué du personnel, non-syndiqué, 29 ans, cadre, Bac+2, PME, demande de licenciement économique)

Rien d'étonnant, dans ce cas, à ce que les relations organisationnelles et les relations affectives puissent être parfois totalement confondues. Le salarié protégé fait alors corps avec l'entreprise ; certains acceptent des mandats électifs moins pour représenter les autres salariés que pour participer au bien de l'entreprise. Ils se dévouent en quelque sorte pour assumer des missions nécessaires ou imposés par la loi. Et souvent leur décision est fortement impulsée par la hiérarchie et par la direction;

\footnotetext{
"Ce qui nous intéressait avec un collègue, c'était le côté comité d'entreprise pas tellement délégué du personnel. Et à ce moment-là, comme personne ne s'est présenté, on l'a fait. Le directeur de l'époque nous y a incité aussi." (Salarié protégé, membre comité d'entreprise, délégué du personnel, non-syndiqué, 29 ans, cadre, Bac+2, PME, demande de licenciement économique)

"(Pourquoi vous dîtes que vous avez été obligée de faire partie du CHSCT ?) Parce qu'en fait il n'y a eu personne dans la société pour se présenter dans ce truc-là. [...] La DRH nous a expliqué qu'à défaut... (Salariée protégée, délégué du personnel, membre du CHSCT, non-syndiquée, 33 ans, CAP, agent de maîtrise, PME, demande de licenciement économique)
} 
Les registres argumentaires des directions sont repris par les salariés pour décrire l'historique de l'entreprise. La responsabilité des dirigeants, par exemple, n'est pas, dans ce cas, interrogée par les salariés. C'est le poids des contraintes qui s'expriment ici et qui pèsent indifféremment sur l'entreprise (la hiérarchie) et les salariés.

1) Un positionnement facilité par l'absence de référence aux organisations syndicales

Ce type de positionnement est particulièrement marqué chez les représentants du personnel qui n'appartiennent pas à une organisation syndicale.

Or, alors que les règles et procédures qui régissent les relations collectives des salariés ont été construites sur l'idée d'une représentation de ceux-ci par les organisations syndicales, force est de constater que la représentation des salariés se fait largement en dehors d'organisations syndicales, donc en dehors d'organisations structurées. Cependant, les statistiques existant sur les candidatures aux élections professionnelles et la ventilation entre syndiqués et nonsyndiqués doivent être lues attentivement. Sur une longue période, qui s'achève en 1991, l'audience syndicale n'a cessé de s'éroder en même temps que le taux de participation baissait. En 1991, presque un tiers des suffrages exprimés se sont reportés sur une liste non syndicale. Depuis, la tendance s'est plutôt inversée et les listes syndicales CGT et CFDT font jeu égal avec les listes non syndicales ${ }^{1}$. Ce retournement de tendance semble en grande partie être conjoncturel, car lié à la mise en place de la réduction du temps de travail et au large mouvement de négociation des accords d'entreprise qu'elle a entraîné. En outre, les informations obtenues dans notre enquête qualitative montrent la présence de non-syndiqués dans des listes syndicales, soit que celles-ci n'arrivent pas à accueillir suffisamment de candidats au sein de chaque organisation, soit que la présentation de candidats non-syndiqués constitue une stratégie d'ouverture de la part des syndicalistes.

De plus, on constate que l'implantation syndicale ou l'audience syndicale est très fortement segmentée : tandis que les grandes entreprises demeurent le terrain d'implantation des organisations syndicales (les listes non-syndiquées n'y représentent qu'un part marginale : $3,2 \%$ des suffrages exprimés en 2001), il en va tout autrement dans les petites entreprises : l'audience syndicale diminue en parallèle à la taille de l'entreprise et pour les entreprises de moins de 50 salariés, les non-syndiqués recueillent plus de la moitié des suffrages $(56,8 \%$ en $2001)^{2}$. Cette segmentation joue aussi, mais à un degré moindre, selon les secteurs d'activité : les non-syndiqués sont, pour ces élections, quasi inexistants dans l'énergie $(1,5 \%)$ ou dans l'industrie automobile $(5,5 \%)$, les activités financières $(6,6 \%)$ et dans l'administration $(7,5 \%)$ alors que leurs suffrages dépassent le tiers dans l'agriculture, sylviculture, pêche $(44,2 \%)$, la construction $(37,9 \%)$ et le commerce $(37,4 \%)^{3}$.

Un autre indicateur révèle la même tendance : sur une longue période, le nombre de conflits se traduisant par une cessation du travail s'inscrit à la baisse : le nombre de jours de grève a été divisé par 4 entre 1975 et 1991 (passant de presque 4 millions de jours de grève à moins d'un million dans le secteur privé) et reste stable depuis, hormis le pic de 1995 (plus de 2 millions de jours de grève $)^{4}$.

\footnotetext{
${ }^{1}$ Le Moigne C. (2003), "Elections aux comités d'entreprise en 2001", Premières informations, octobre 2003 n 43.2.

${ }^{2}$ Ibid.

${ }^{3}$ Ibid.

${ }^{4}$ Merlier R. (2003), "Les conflits en 2001 : une légère baisse", Premières informations, août 2003 n³4.1.
} 
Pour autant, ce basculement de la représentation syndicale vers des listes non-syndiquées, tout comme la diminution des grèves, ne signifie pourtant pas que nous entrons dans une ère de paix et de consensus entre les salariés et leurs employeurs. Les nombreux débats autour de la souffrance au travail ou encore l'inscription, dans la loi, de la notion de harcèlement attesteraient plutôt du contraire. Plus certainement, les formes de représentation, les références des individus qui y postulent, comme les affrontements entre salariés et dirigeants doivent être ré-analysés à partir de notions et d'outils différents de ceux qui étaient utilisés pour comprendre les rapports salariés - dirigeants dans la période où le modèle fordiste servait de référence. Tandis que des pans entiers de l'économie et du salariat étaient très loin de s'inscrire dans ce modèle, c'est pourtant sur lui que se focalisaient les études sur la représentation syndicale et sur l'analyse des rapports antagonistes salariés-patronat.

\section{2) Des références personnelles à l'intérêt collectif}

\section{Le choix des "gens" et la méconnaissance ou le rejet des syndicats}

On ne doit pourtant pas comprendre ce mode d'occupation comme une orientation purement individualiste ou utilitaire de certains salariés. Les salariés protégés font en effet souvent référence à un intérêt collectif, mais il est très faiblement formalisé et se décrit de manière vague.

\footnotetext{
"J'avais envie d'aider les gens tout simplement [...] Quelqu'un qui a un problème, pouvoir l'aider avec mes maigres moyens, pouvoir lui accorder du temps et essayer de solutionner ça, ça a toujours été mon rêve de faire du social, c'était un moyen comme un autre.» (Salariée protégée, délégué du personnel, membre du CHSCT, non-syndiquée, 33 ans, CAP, agent de maîtrise, PME, demande de licenciement économique)
}

"Il n'était pas affilié à un syndicat. Il était très dans l'esprit (maison) qui est : je fais quelque chose de collectif parce que ça aide tout le monde, ça aide l'entreprise, les salariés, si je peux être utile, etc... C'était ça son thème à lui, ce n'était pas idéologique au sens français du mot. [...], Je pense qu'il avait un idéal. Il était dans cette mouvance, je pense qu'il avait vraiment l'impression de rendre service à la collectivité, non, je pense que contrairement à ce qu'on pourrait imaginer dans un décodage assez basique, je pense que ce n'était absolument pas quelqu'un qui était là pour négocier dans son intérêt personnel." (DRH, grande entreprise)

Par contre, la position de ces salariés est le plus souvent construite en dehors de toute référence à l'organisation syndicale et même parfois comme un rejet des organisations syndicales.

Tout d'abord, le nombre de représentants du personnel non-syndiqués est relativement important. L'enquête REPONSE, par exemple, indique que le taux de syndicalisation des principaux représentants du personnel est de 50,4\% mais seulement de $22,8 \%$ pour les représentants élus et $20,5 \%$ pour les seuls secrétaires de comité d'entreprise ${ }^{1}$.

Dans notre l'enquête, certains manifestent une méconnaissance du fait syndical assez étonnante. Tous les syndicats apparaissent comme une nébuleuse. C'est le cas, par exemple,

\footnotetext{
${ }^{1}$ Furjot D. (2002), De la participation au conflit. Enquête "réponse" 1998. Questionnaire "représentants du personnel", Document d'études n64, Déc. 2002, DARES.
} 
d'un salarié protégé qui croit que son collègue CGC est militant de la CGT. Comme le syndicat est par essence étranger à leurs valeurs, aucune distinction n'est faite entre les différents syndicats et entre leurs positions.

Plus surprenant encore, les non-syndiqués candidats sur des listes syndicales sont parfois hésitants pour citer la liste qui les a accueillis

\footnotetext{
"Je n'étais pas syndiquée, mais j'ai été élue sur une liste syndicale. (Quel syndicat ?) Je crois que c'est la CFDT, si je ne me trompe pas.» (Salariée protégée, délégué du personnel, membre du CHSCT, non-syndiquée, 33 ans, CAP, agent de maîtrise, PME, demande de licenciement économique)
}

Même présentés par une liste syndicale, ils n'adhérent pas tous à l'organisation qui fédère la liste.

\begin{abstract}
"(Vous étiez proche des syndicats avant ?) Franchement je m'en fichais, c'est un moyen comme un autre d'atteindre le but qui est le social pour moi. [...] On connaissait une des deux personnes qui était syndiquée et qui montait la liste syndicale, elle avait besoin de personnes, je ne vais pas dire de confiance. [...] et donc on s'est retrouvé comme ça sur la liste syndicale. Parce qu'au $1^{\text {er }}$ tour, on nous avait dit qu'il ne pouvait y avoir que la liste syndicale et pas de liste (non-syndiquée).» (Salariée protégée, délégué du personnel, membre du CHSCT, non-syndiquée, 33 ans, CAP, agent de maîtrise, PME, demande de licenciement économique)
\end{abstract}

Souvent c'est sur la tonalité du rejet que s'expriment les salariés quand ils parlent du syndicat.

"Je ne suis ni syndicaliste de gauche ou de droite ou de quoique ce soit, je suis tout à fait indépendant dans ce domaine-là.» (Salarié protégé, secrétaire du CHSCT, non-syndiqué, 55 ans, cadre, BTS, CE-CHSCT, grande entreprise, licenciement économique)

Le syndicat est en effet rejeté pour plusieurs raisons qui forment, dans les argumentations des salariés non-syndiqués, un système relativement cohérent. Tout d'abord par sa composante idéologique : chaque organisation syndicale se réfère à un corpus relativement bien balisé qui constitue une contrainte que rejettent ces salariés protégés.

"Nous voulions absolument être neutres, vis-à-vis de la direction et vis-à-vis des syndicats, parce qu'autrement, il y a l'effet pervers. L'effet pervers de se retrouver bloqué par un syndicat et de suivre ses pensées qui vont peut-être à l'encontre d'un autre syndicat.» (Salarié protégé, secrétaire du CHSCT, non-syndiqué, 55 ans, cadre, BTS, CE-CHSCT, grande entreprise, licenciement économique)

"Ce sont des positions strictement personnelles ou religieuses que j'ai, je considère que toutes les personnes et les syndicats sont bons, et donc je travaille avec tous les syndicats.» (Salarié protégé, secrétaire du CHSCT, non-syndiqué, 55 ans, cadre, BTS, CE-CHSCT, grande entreprise, licenciement économique)

En outre, le rattachement à une organisation va, compte tenu des doctrines que les militants sont censés intégrer, à l'encontre de la liberté individuelle ; il apparaît comme un frein aux relations inter-individuelles "vraies"

\footnotetext{
"Je n'ai jamais adhéré à aucun parti, je suis toujours resté assez libre et de plus en plus désenchanté d'ailleurs. [...] Dans l'entreprise, ça ne m'était jamais venu à l'idée, et puis quand on s'est syndiqué c'était uniquement pour être réglo, on n'en avait rien à foutre des syndicats, on ne savait même pas comment ça marchait vraiment, on était même assez sceptique par rapport à tout
} 
ça. On avait une image des syndicats qui n'était pas très bonne, pas très valorisante ${ }^{1 "}$ (Salarié protégé, membre du comité d'entreprise, délégué du personnel, syndiqué, 37 ans, cadre, Bac+2, PME, demande de licenciement pour faute)

La plupart de ces salariés présentent donc leur candidature comme a-conflictuelle et, la plupart du temps, assez nettement opposée aux syndicats.

"Je n'ai jamais eu une grande sympathie pour les syndicats, ce que j'en pense de la communication
des syndicats et tout ça, ce n'est pas tellement dans ma culture, je n'étais pas très syndicat (Salarié
protégé, membre comité d'entreprise, délégué du personnel, non-syndiqué, 29 ans, cadre, Bac+2,
PME, demande de licenciement économique)

Mais leur choix de s'investir dans le "social" se réfère pourtant à une conception du bien commun, même si elle est formulée de manière vague et qu'elle fait le plus souvent références aux relations inter-individuelles. L'utilisation du terme "gens", en lieu et place des appellations "salariés" ou "travailleurs", plus habituelles dans le monde syndical, marque l'ambiguïté du collectif de référence, mais, pour autant, n'indique pas sa disparition en tant que référence.

\begin{abstract}
"Je passais plus auprès des gens, je pense que le côté, j'ai d'être assez proche des gens et de ne pas vouloir rentrer en conflit. Les gens me connaissaient depuis longtemps et je pense que je passais bien à tous points de vue par rapport à eux, c'est-à-dire ne pas chercher à semer la zizanie juste pour la semer. Je pense que c'est parti sur un climat de confiance de la part des salariés et d'un relationnel probablement qui faisait que les gens ont adhéré à ma candidature.» (Salariée protégée, déléguée du personnel, non-syndiquée, 55 ans, profession intermédiaire, sans diplôme, TPE, demande de licenciement économique)

"Nous en tant que DP, on est là pour aider les gens, on n'est pas là pour se mêler de la gestion de la société.» (Salariée protégée, délégué du personnel, membre du CHSCT, non-syndiquée, 33 ans, CAP, agent de maîtrise, PME, demande de licenciement économique)
\end{abstract}

L'entrée dans le mandat n'est donc pas motivée, en premier lieu, par la volonté de représenter les salariés ; il s'agit plutôt, pour ces salariés protégés, d'aider les autres salariés, sans que l'on sache très bien ce que recouvre cette notion. L'ambiguïté de cette orientation laisse ainsi de l'espace à la poursuite d'objectifs complémentaires dont ils définissent les contours et que nous allons voir maintenant.

\title{
Le parti de l'entreprise contre les intérêts "égoïstes" des salariés
}

Ce relatif rejet des organisations syndicales se double d'une valorisation de l'entreprise. Celleci apparaît, au départ, pour ces salariés, non comme un terrain conflictuel mais beaucoup plus comme un espace de réalisation de soi. Ainsi les rapports sont bons et parfois même, excellents.

"Les rapports étaient bons, ça c'est un peu dégradé après." (Salarié protégé, membre comité d'entreprise, délégué du personnel, non-syndiqué, 29 ans, cadre, Bac+2, PME, demande de licenciement économique)

Il est d'ailleurs tout à fait marquant qu'au moment de postuler à l'embauche, des salariés s'interrogent tant sur les qualités de l'entreprise que sur les contreparties salariales

\footnotetext{
${ }^{1}$ En fait, ce salarié va évoluer graduellement et se retrouver par la suite Délégué syndical.
} 
"Rapidement je me suis rendu compte que c'était un boulot sympa, un produit agréable, les clients plutôt gentils. [...]Il n'y a aucun souci, j'adhère complètement, en plus le concept a été plutôt bien pensé, on est dans un créneau très mal exploité et dans une gamme de produits qui est vraiment le cœur du marché.» (Salarié protégé, membre du comité d'entreprise, délégué du personnel, syndiqué, 37 ans, cadre, Bac+2, PME, demande de licenciement pour faute)

L'adhésion à l'entreprise se voit aussi par l'emploi du pronom indéfini "on"

"C'est une société familiale qui s'est développée extrêmement vite. En à peine 20 ans, on est passé d'un à 82 magasins" (Salarié protégé, membre du comité d'entreprise, délégué du personnel, syndiqué, 37 ans, cadre, Bac+2, PME, demande de licenciement pour faute)

Il n'est pas étonnant que des salariés non-syndiqués se présentent aux élections encouragés par leur hiérarchie

"Et à ce moment-là, comme personne ne s'est présenté, on l'a fait. Le directeur de l'époque nous y a
incité aussi, il nous a dit que ce n'était pas bien qu'il n'y ait personne, il fallait que quelqu'un se
présente." (Salarié protégé, membre comité d'entreprise, délégué du personnel, non-syndiqué, 29
ans, cadre, Bac+2, PME, demande de licenciement économique)
"Un exemple typique c'est (le directeur qui dit) : je verrai bien un tel et un tel comme délégué
comité d'entreprise. [...] On a l'impression que c'est lui qui pourrait les désigner." (Secrétaire du
comité d'entreprise, syndiqué, PME)

Ce positionnement provoque parfois une certaine confusion ou un tiraillement pour des salariés qui se vivent comme porteurs de l'intérêt de l'entreprise qui est pour eux aussi une sorte d'intérêt collectif qui s'oppose aux intérêts individuels.

"Honnêtement, j'ai trouvé (l'accord RTT) extrêmement satisfaisant pour les salariés, de mon point
de vue, même trop. Je faisais partie du collège cadre et agent de maîtrise, mais j'étais plus du côté
employeur, et c'est vrai que ça a augmenté très fortement la masse salariale, tant mieux pour les
salariés mais ça a coûté cher à l'entreprise." (Salarié protégé, membre comité d'entreprise, délégué
du personnel, non-syndiqué, 29 ans, cadre, Bac+2, PME, demande de licenciement économique)

La recherche d'une valorisation personnelle

Compte tenu de l'absence de normes de comportement ou de contraintes imposées par l'organisation syndicale sur les raisons et la manière d'occuper le mandat, ces salariés protégés peuvent ainsi s'investir pour des raisons plus personnelles qui peuvent être résumées par la recherche d'une valorisation sociale et professionnelle.

Dans un tel cadre, les nouvelles tâches incluses dans le mandat apparaissent, dès lors qu'elles ont pour effet d'enrichir l'individu, déterminantes dans le choix d'occuper ou non le mandat.

"Pour moi c'était une chance d'apprendre encore plus.» (Salariée protégée, déléguée du personnel, membre du CHSCT, non-syndiquée, 33 ans, CAP, agent de maîtrise, PME, demande de licenciement économique)

Mais cette valorisation ne se réduit que rarement à une simple acquisition de connaissances techniques et à un enrichissement personnel du salarié. Le mandat représente en effet surtout un moyen d'acquisition de leur propre "valeur", les apprentissages ou les nouvelles fonctions n'étant qu'un moyen parmi d'autre d'atteindre ces objectifs. Les rapports entre individu et 
travail deviennent, dans cette optique, tout à fait centraux. En effet, le travail - ici dans son sens le plus large ou le plus commun, d'activité salariée ou non - sert de référence identitaire (Castel, 1995).

Pour ces salariés protégés, l'occupation du mandat est inséparable de la manière dont ils conçoivent leur activité professionnelle, déniant, de ce fait, toute composante idéologique au mandat qui est simplement perçu comme une extension du poste occupé jusqu'alors et doté d'un contenu fonctionnel. C'est ce qui explique que ce sont les mandats les plus techniques qui sont investis par les salariés qui s'inscrivent dans cette logique.

En outre, le rôle identitaire du travail ne tient pas uniquement à son existence mais aussi à sa dynamique. Intégrant de multiples dimensions individuelles, et notamment celles qui sont liées à la réalisation de soi ou à la reconnaissance sociale, le travail ne fait pas sens pour les individus par sa seule existence ou ses contreparties salariales mais par la possibilité qu'il offre de modifier leur devenir (Berton, Correia et alii, 2004). L'engagement dans un mandat permet ainsi de passer d'une position d'exécution à une position où la marge de décision n'est pas négligeable. L'engagement permet d'accroître les capacités d'intervention de l'individu en augmentant l'accès aux informations, mais aussi la marge d'autonomie dont il dispose. L'engagement réduit fortement les contraintes et permet à l'individu d'effectuer des choix de champs et de modes d'action. Et surtout ce type d'engagement repositionne l'individu de sujet à acteur de l'organisation et de sa propre vie.

Toutefois, à moyen et long terme, se pose obligatoirement la question de la reconnaissance de cette valeur par l'entreprise et donc son inscription dans les hiérarchies de prestige de l'entreprise, c'est-à-dire la possibilité d'obtenir une promotion professionnelle. Mais très souvent après une phase d'apprentissage parce que l'augmentation de compétences, de responsabilités ne se traduit pas symboliquement dans un changement de fonction et surtout de niveau, l'individu parfois très maladroitement, essaie de convaincre ses interlocuteurs de cette augmentation de compétences.

Toutefois, le fait que l'occupation du mandat soit conçue comme une fonction purement individuelle, sans appartenance ou même référence à une organisation syndicale place les salariés en position de relative fragilité.

\section{3) Les déterminants des candidatures individuelles}

Ce mode d'occupation du mandat aurait été beaucoup plus difficilement envisageable dans la première moitié du siècle, ne serait-ce que parce que les militants syndicaux étaient plus nombreux, que les organisations syndicales contrôlaient mieux le jeu de la représentation, qu'elles disposaient de suffisamment de militants pour occuper des mandats qui existaient en nombre beaucoup plus réduit. En outre, la pression morale, la capacité à normaliser les comportements des salariés que détenaient les organisations syndicales étaient beaucoup plus importants. 


\section{Déterminants structurels}

Toutefois, ce sont certainement les évolutions socioprofessionnelles qui sont les plus éclairantes pour comprendre l'apparition et le développement de ce type de relations aux mandats et aux situations professionnelles.

Le centre de gravité de la société s'est aussi déplacé vers les cols blancs et les professions intermédiaires. Les contenus des activités changent, comme les conditions dans lesquelles les personnes accèdent à un emploi, et celles dont elles bénéficient quand elles en occupent un. Les activités deviennent beaucoup moins précises. Ces changements brouillent très fortement les repères professionnels sur lesquels les individus pouvaient se construire des projets d'évolution professionnelle.

Cette évolution a été décrite par Mendras (1988) comme une "moyennisation de la société" c'est-à-dire un monde social structuré autour des deux constellations que sont la constellation populaire (ouvriers et employés proches par le bas niveau de diplôme et par le faible revenu) et la constellation centrale (caractérisée par une mobilité sociale intense) dans lesquelles les individus montent normalement en grade, en dignité et en revenu à mesure qu'ils avancent en âge.

En effet, l'ensemble des positions professionnelles s'est plutôt déplacé vers le "haut" ${ }^{1}$. En moins d'une décennie, entre 1982 et 1990, la population des cadres et professions intellectuelles supérieures ainsi que celle des professions intermédiaires a ainsi augmenté respectivement de 40 et $17 \%$ (alors que le nombre d'employés n'augmentait que de $7 \%$ - mais sur un stock beaucoup plus important au départ - et celui des ouvriers baissait de $7 \%^{2}$ ). Représentant un quart de la population active en 1982, les cadres et les professions intermédiaires en forment le tiers huit ans plus tard.

L'analyse de Mendras, critiquée car elle affirmait implicitement la fin de la société de classes, rend pourtant compte de la disparition de la classe ouvrière comme pôle de référence. Or les employés, dont le nombre n'a cessé de croître tout au long du siècle (cf. Marchand et Thélot), présentent une hétérogénéité beaucoup plus forte que les ouvriers, "Les employés de bureau, archétypes de ce secteur, apparaissent comme une masse indifférenciée présentant une faible cohérence et dont il est difficile de percevoir la spécificité de leur activité." (Chenu, 1990).

Rien d'étonnant, alors, à ce que l'identification à un collectif - c'est-à-dire les distinctions sociales antérieures : classes sociales traditionnelles, métiers, anciennes organisations - se réduise (Sainsaulieu, 1990) alors même que certains salariés demandent à négocier de manière individuelle, sans le soutien du collectif ou de ses représentants (Correia \& alii, 1999). En outre, Mendras en partant du constat que le patrimoine économique est remplacé par un patrimoine de compétences, la richesse par le savoir, rend compte de nouveaux modèles de référence professionnels congruents au mode d'occupation du mandat et de relation à la situation professionnelle décrite ici.

\footnotetext{
${ }^{1}$ Il s'agit ici de positions professionnelles. Comme cela a déjà été dit, l'augmentation de ces positions affecte leurs positions sociale relative.

${ }^{2}$ Maurin E., "Les transformations du paysage social dans les années quatre-vingt", in "La société française. Données sociales 1993", I.N.S.E.E., 1993, pp. 458-465.
} 
L'entreprise devient, en ce sens, un des référents individuels parmi d'autres, mais moins conflictuel qu'auparavant. Or, on constate dans le même temps une évolution des modes de management des entreprises : dans un nombre significatif d'entre elles, notamment dans les nouveaux secteurs qui ne connaissent pas de tradition syndicale, le management tente de mettre en œuvre un "management intégratif" (Maggi-Germain, Correia, 2001) dont nous résumons ici quelques traits.

\begin{abstract}
Un nouveau type de management tend aujourd'hui à s'imposer. Il demande aux salariés de s'identifier à l'entreprise et à ses objectifs; on peut, de ce fait, le qualifier de management intégratif. À l'opposition entre les intérêts des salariés, pris collectivement, et ceux de l'entreprise, succède la fusion des intérêts de l'individu avec ceux de l'organisation. Certaines entreprises proposent ainsi, à leurs salariés, des services qui faisaient autrefois partie intégrante de la vie extra professionnelle, (salle de repos ou de gymnastique, crèches...) ou encore intègrent dans une activité professionnelle des activités normalement considérées comme extra professionnelles (stages culturels, formation en internat facilitant les relations affectives, séjours au sport d'hiver avec chaque famille...). Les frontières du travail s'en trouvent ainsi brouillées. [...] On peut, dans un premier temps, penser que ce nouveau mode de management ne fait que reproduire un mode déjà ancien de gestion des relations de travail de type paternaliste. Il n'en est pourtant rien. En effet, la gestion paternaliste se fondait, pour sa part, sur l'adhésion des salariés à la figure du "patron". Cette personnalisation du pouvoir structurait la relation de travail sur un modèle hiérarchique. À l'inverse, en présentant les relations professionnelles comme des relations égalitaires, le management intégratif se distingue, malgré de nombreux points communs, du paternalisme. On ne recherche plus l'adhésion des salariés au patron-bienfaiteur, mais à l'entreprise-institution. Plutôt que d'une réelle égalité, il s'agit d'ailleurs plus de la mise en place de signes d'indifférenciation comme, par exemple, des tenues vestimentaires très proches, quelle que soit, par ailleurs, la position occupée dans l'entreprise. Le discours tenu se veut rassembleur, gommant implicitement les différenciations (l'appel à la participation à un projet commun). La référence au travail comme une participation à une aventure commune est parfois clairement revendiquée. L'entreprise à laquelle appartient le salarié devient ainsi la valeur de référence. Dans sa forme la plus «aboutie», c'est-à-dire lorsque le salarié devient actionnaire de l'entreprise, ses intérêts se confondent alors avec ceux de l'organisation. Les décisions managériales peuvent alors être transformées en projets communs et faire l'objet d'une "responsabilisation" individuelle. (Maggi-Germain, Correia, 2001).
\end{abstract}

Ce modèle est loin d'être général, mais ces composantes sont largement diffusées sous des formes plus flexibles de participation qui font appel à l'expression directe des salariés selon la nature des problèmes à résoudre (amélioration des dispositifs techniques, modification des conditions de travail, réduction du nombre de plaintes de la clientèle, etc...). Comme le note Tixier (1992), on voit alors se développer une vive opposition entre la logique de mobilisation des masses, caractéristique de l'action syndicale traditionnelle, et la nouvelle logique participative, qui ne concerne plus que des micro-collectifs dociles, intériorisant les contraintes de la production.

Nous ne disposons pas de données quantitatives, mais il est probable que ce type de consensus tend à se développer, non seulement dans les entreprises de la nouvelle économie, mais aussi dans les PME au sein desquelles les formes d'intégration s'apparentent plus au modèle traditionnel que constitue le paternalisme qu'au management intégratif.

Plusieurs analyses convergent ainsi pour relier la progression des licenciements pour motif personnel (qui sont une autre manière de constater une personnalisation des fonctions) à des pratiques nouvelles de gestion de l'emploi et des effectifs : "les licenciements pour motif personnel seraient l'un des moyens de réduire ou de recomposer la main d'œuvre dans le cadre 
de restructurations" (Pignoni et Zouary, 2003) ${ }^{1}$. Et les auteurs citent la diffusion de formes de management par objectifs, caractérisées par une gestion de l'emploi plus individualisée, une plus grande autonomie et responsabilisation des salariés dans l'exécution du travail, mais aussi des formes de contrôle plus systématiques et individualisées et des obligations de résultat plus contraignantes. Et comme le disent encore les auteurs ces nouvelles contraintes échappent pour l'instant à l'analyse juridique.

Mais les volontés patronales seraient bien en peine d'imposer des nouveaux modes de régulation si ceux-ci ne rencontraient des salariés, bien sûr pas tous, tout disposés à intégrer ces nouvelles règles du jeu.

La démocratisation de l'enseignement - et notamment de l'enseignement supérieur - a profondément modifié le niveau de diplôme détenu par les salariés. Les jeunes entrant sur le marché du travail disposent maintenant de niveaux de formation très nettement supérieurs à leurs aînés qui ont commencé à travailler il y a 30 ou même 20 ans $^{2}$.

Le premier effet de cette élévation considérable du niveau de diplôme détenu par la population active est la production, par l'institution scolaire, de diplômes en nombre plus important que ce que peut absorber le marché de l'emploi (Pottier, 1992) ${ }^{3}$.

Le deuxième effet est une massification de la scolarisation qui modifie la valeur relative des diplômes. En effet, lorsque la quasi-totalité d'une classe d'âge parvient au niveau Bac et que, en outre, les étudiants ont de plus en plus tendance à poursuivre leurs études, y compris dans les filières techniques (Dubois, 1993), les entreprises peuvent, dans tous les cas, recruter de jeunes débutants formés et "surqualifiés" plutôt que promouvoir ceux qui ont acquis leurs compétences sur le tas. Pour arriver à «percer» dans le milieu professionnel, il faut affronter des concurrents disposant des mêmes ressources (Bauer, 1987).

Or, en même temps que la scolarité initiale fournit des diplômes de plus en plus élevés à ceux qui en sortent, le chômage touche une part de plus en plus importante de la population active ${ }^{4}$.

Cette dégradation du marché du travail est concomitante à une précarisation importante des statuts d'emploi. Depuis le début des années 80, les entreprises ont considérablement développé le recours à l'intérim et aux contrats à durée déterminée pour ajuster leurs effectifs : le nombre de personnes employées sur ce type de contrat a doublé entre 1981 et 1994 et représente désormais près de $6 \%$ des salariés des secteurs marchands non agricoles.

\footnotetext{
${ }^{1}$ Pignoni M-T., Zouary P. (2003) "Les nouveaux usages du licenciement pour motif personnel", Premières informations, juillet $2003 \mathrm{n}^{\circ} 28.2$.

${ }^{2}$ En 1993, plus d'un tiers des Français de 25 à 39 ans sont bacheliers contre un cinquième seulement des 50-59 ans. Source : Enquête FQP 1993, INSEE

${ }^{3}$, Jusqu'à la fin des années 70, la production de diplômes de niveau I à III (supérieur à Bac +2 ) était inférieure au nombre de recrutements de jeunes débutants ingénieurs, cadres et professions intermédiaires réalisés par les entreprises. À partir du début des années 80, la proportion s'est inversée : le nombre de diplômés est devenu supérieur au nombre de postes. Cet écart n'a fait que s'accroître (cf. F. Pottier et S. Zilbermann, «La difficile insertion professionnelle des jeunes, Céreq-Bref, oct. 1990).

${ }^{4}$ Pour se rendre compte de l'importance de cette transformation, il faut se rappeler que, sur la période allant de la seconde guerre mondiale aux années 70, le taux de chômage n'a jamais dépassé 2,2\%. (Source Enquêtes emploi, INSEE Résultats n62 - 63, "Marché du Travail, séries longues", INSEE, 1994). Ce taux représentait seulement le temps minimum nécessaire pour passer d'un poste à un autre. En mars 1996, ce taux était six fois supérieur (Source Enquête Emploi mars 1996, "Emploi et chômage en mars 1996", INSEE première n 467, Juin 1996).
} 
De plus, cette précarisation se réalise parallèlement au développement d'autres formes d'emploi, notamment du temps partiel, qui distendent les liens des salariés avec leur organisation et contraignent leur évolution professionnelle. C'est, au bout du compte, quand on totalise les situations particulières d'emploi "nouvelles" (CDD, Intérim, temps partiel, mesures jeunes et non-titulaires de la Fonction publique), quasiment un salarié sur cinq qui est concerné par une forme atypique d'emploi ${ }^{1}$.

Tout cela dessine un nouveau contexte social dans lequel le rapport au travail et aux employeurs devient plus complexe, multipliant à l'infini les formes que peut prendre une carrière professionnelle tout en rendant l'individu initiateur de cette évolution. Cela implique, pour les individus, un investissement professionnel quasi obligatoire, sous peine de se retrouver rapidement sur la touche.

Or ces évolutions socioprofessionnelles accompagnent et ont aussi en grande partie pour effet, une individualisation ${ }^{2}$ des sociétés occidentales.

Ce mouvement d'individualisation est porté par un certain nombre d'évolutions en partie déjà citées ci-dessus: l'élévation du niveau de vie, l'expansion des positions professionnelles situées en haut de la hiérarchie, l'augmentation de la mobilité professionnelle et géographique participent à la diminution des liens de parenté ou de voisinage. En même temps, la généralisation du modèle culturel d'efficacité et de rationalité, l'augmentation du niveau de formation promeuvent la représentation d'un individu autonome et rationnel, nouvelle figure emblématique appelée à remplacer les anciennes figures mythiques comme celle de l'ouvrier industriel (Berton, Correia \& alii, 2004). Il est alors possible de constater que la consistance individuelle prend de l'épaisseur, et que les individus sont, plus qu'avant, dotés de capacités réflexives qui permettent de comprendre l'initiative individuelle comme une décision dotée d'une certaine rationalité même si celle-ci est loin de résumer la totalité des actes individuels.

Les approches analytiques récentes renvoient aussi à une difficulté plus subtile. Le constat de la place centrale de l'individu va de pair avec la difficulté qu'a celui-ci à exister : l'unification de l'acteur en présence d'un monde de plus en plus complexe et diversifié est problématique. L'individu devient alors, plus que le collectif, une référence centrale, une norme de comportement, mais cela se traduit aussi par une exposition beaucoup plus importante des individus qui deviennent beaucoup plus fragiles dans une "société de verre", selon l'expression de Philippe Corcuff (2002).

Il convient, toutefois, de différencier les populations concernées : cette individualisation et cette exposition concernent avant tout les populations les plus qualifiées et les plus diplômées.

\footnotetext{
${ }^{1}$ En 1993, 2280000 salariés occupaient un emploi à temps partiel contre 788000 salariés en 1973. (Source Enquête Emploi et recensement des agents de l'Etat in INSEE, "Données sociales 1996", INSEE). Ces nouvelles contraintes concernent, avant tout, les jeunes : d'une part, ils sont les plus touchés par le chômage : la part de chômeurs parmi les jeunes de 15 à 29 ans est de $21,7 \%$, alors que ce taux n'est que de $12,1 \%$ pour l'ensemble de la population active. (Source "Enquête sur l'emploi de 1996", INSEE Résultats n492-493, Septembre 1996) d'autre part, ce sont eux aussi qui sont les plus affectés par les formes de travail précaire : en 1993, ces CDD, Intérim, temps partiel, mesures jeunes et non-titulaires de la Fonction publique représentaient 4666000 personnes. (Source Enquête Emploi et recensement des agents de l'Etat in INSEE, ibid);.

2 La question n'est toutefois pas nouvelle, Durkheim (1893), en analysant le passage de la solidarité mécanique à la solidarité organique, ou Weber (1922), avec le passage de la communalisation à la sociation, pointent l'émergence d'un individu moins contraint par son contexte social. Pour une approche des difficultés liées aux différents sens de la notion d'individu et de la littérature, appliqué à la formation mais qui est largement transposable à d'autres domaines, cf. Berton F., Correia M., Maillebouis M., Lespessailles C. (éds). (2004), "La demande de formation : quelle place à l'initiative individuelle?", l'Harmattan.
} 
Ceci est concordant avec un certain nombre d'évolutions des causes de licenciement relevées par les études menées au sein du Ministère du travail. C'est en effet parmi les cadres et les agents de maîtrise que le licenciement pour motif personnel est le plus fréquent ${ }^{1}$. Le lien entre ces deux types de phénomènes est clairement fait par Pignoni et Zouary : les cadres, échappant plus souvent que les salariés moins qualifiés à la logique syndicale en matière de relations professionnelles, adoptent des stratégies plus individualistes, y compris dans les secteurs comme la banque où le taux de syndicalisation reste globalement élevé. Nombre de ces licenciements pour motif personnel peuvent ainsi traduire une stratégie de négociation préalable au départ de l'entreprise. Par ailleurs, le management par objectifs et l'individualisation de la main-d'oeuvre sont des pratiques qui touchent prioritairement les catégories et les secteurs correspondant à ces familles professionnelles ${ }^{2}$.

\section{Déterminants individuels}

Les caractéristiques des salariés eux-mêmes sont aussi déterminantes pour comprendre ce type de positionnement.

Ce sont, en premier lieu, les salariés les plus jeunes qui placent dans le monde du travail en général et dans l'entreprise en particulier un certain nombre d'attentes très différentes de celles que pouvaient formuler leurs prédécesseurs. Ce constat s'explique en partie, par leur niveau de diplôme, très nettement supérieur ${ }^{3}$ et, d'autre part, parce qu'ils se trouvent souvent à des postes d'un niveau inférieur à celui auquel ils auraient pu prétendre.

De ce fait, mais aussi en l'absence de possibilités réelles de reclassement dans une autre organisation, par crainte du chômage ou parce que les avantages de l'organisation (sécurité d'emploi, horaires...) demeurent importants, certains de ces jeunes surdiplômés, se placent volontairement en situation de retrait par rapport à l'organisation, dans ce que l'on peut qualifier, pour reprendre les termes d'Hirschman (1972), d'"exit" . Ils n'ont alors que peu de raisons de postuler à des mandats représentatifs.

D'autres salariés protégés réagissent de manière plus offensive ${ }^{4}$. Ils sont demandeurs de situations de travail plus intéressantes et donc complexes et tentent de négocier directement avec l'encadrement des fonctions spécifiques et/ou d'élargir les limites des fonctions qu'ils occupent déjà (Correia, 2003). Dans cet accord, les jeunes se voient offrir des fonctions beaucoup plus intéressantes, car bénéficiant d'une grande autonomie dans l'organisation du travail et d'une importante responsabilité dans les décisions éventuelles à prendre. On est alors loin de la caricature des salariés prêts à tout pour parvenir au sommet de la hiérarchie, auxquelles on rattache les termes de carrière, l'engagement dans l'entreprise ne pouvant pas être ici confondu avec le carriérisme.

\footnotetext{
${ }^{1}$ Pignoni M-T., Zouary P. (2003) "Les nouveaux usages du licenciement pour motif personnel", Premières informations, juillet $2003 \mathrm{n}^{\circ} 28.2$.

${ }^{2}$ Ibid

${ }^{3}$ Les sorties nettes de l'enseignement supérieur au niveau I+II de formation (licence et plus) qui représentaient en 1980, 9,5\% des sortants du système éducatif, en représentent 19,4\% en 1992. (J. Vincens, F. Pottier, Le devenir des diplômés des Universités, Rapport au Comité National d'Evaluation des établissements publics à caractère scientifique culturel et professionnel, Janvier 1995).

${ }^{4}$ Une partie des analyses présentées ici a été d'abord étayée à partir d'une enquête sur les "surdiplômés" agents du Ministère de l'équipement et qui permettent de généraliser et de condenser les résultats auxquels on parvient ici sur un nombre moins élevé d'entretiens. (Correia M. (dir.), Leroux N., Lenel P., "Entre désinvestissement, promotion et mobilité diagonale : le cas des "surdiplômés" du Ministère de l'équipement, du logement, des transports et du tourisme", doc. interne, Ministère de l'équipement, du logement, des transports et du tourisme, 158 pages.
} 


\begin{abstract}
"Je pense que d'abord, $\mathrm{X}$ n'était pas un ambitieux, il ne voulait pas faire carrière, ce qui le préoccupait, c'était de faire quelque chose qui l'intéressait. C'était un fêlé d'informatique, [...] quand il a mis en place l'informatique de la qualité de tout le groupe, ça, ça commençait vraiment à l'intéresser." (Directeur d'établissement, PME)
\end{abstract}

"Il avait mis lui en informatique toutes les procédures de qualité, ça l'avait beaucoup excité cette histoire, ça lui plaisait." (Directeur d'établissement, PME)

Ceci n'empêche pas bien au contraire que leur sort dans l'entreprise apparaisse enviable.

\begin{abstract}
"Je faisais pas mal d'informatique, parce qu'en fait j'ai pris des cours de BTS Informatique par le CNED. Et ça je le faisais pendant que j'étais à l'armée. (Après dans l'entreprise) J'ai évolué quand même très rapidement, en fait, quand j'ai commencé, j'étais vraiment à l'échelon le plus bas dans la convention collective, et 4 ans après, j'étais cadre. (Pendant ce temps) j'ai repris mes études et j'ai passé un DUT de gestion en formation continue. [...] Mon titre c'était informaticien à l'époque, et là, il y a 2 ans quand je suis passé cadre, mon titre est devenu celui de responsable développement." (Salarié protégé, membre comité d'entreprise, délégué du personnel, nonsyndiqué, 29 ans, cadre, Bac+2, PME, demande de licenciement économique)
\end{abstract}

Toutefois, dès lors que la nature des fonctions n'est pas totalement définie, - l'autonomie est plus une question de degré que d'état - le système d'échange intégré dans le contrat ne peut faire l'objet d'un accord totalement explicite. Il s'agit alors, selon les termes de Saglio (1991), d'un "échange social" qui fait appel à la notion d'engagement, de mobilisation. Or, contrairement à l'échange économique dans lequel les obligations des uns et des autres sont entièrement spécifiées à l'avance, intervient ici, dans le cours de la transaction, un phénomène de «réciprocité »: les promesses inscrites implicitement au contrat vont s'échanger dans le temps.

Que l'échange ne soit pas instantané et qu'il intègre un aspect qualitatif important, rend le contrôle de l'échange particulièrement difficile. De plus, que l'autonomie fasse partie de l'échange rend le contrôle tout à fait inopérant puisque cela reviendrait à nier justement la nature de ce qui est engagé. La réciprocité inhérente à ce type d'échange impose alors d'ajouter à la relation initiale, qui se fait entre des positions, la confiance qui est une notion qui ne peut s'appliquer qu'à des personnes. Le contrat moral engagé ici n'est donc pas contraire à la subordination, mais il ne peut non plus totalement s'y résumer.

\footnotetext{
"On m'a dit que c'était intéressant, qu'on était assez libre, parce que comme pendant une dizaine d'années, j'étais indépendant, je ne voulais pas me retrouver dans une société où il y avait des comptes à rendre au jour le jour, où on avait des gens sur le dos au niveau de la hiérarchie. [...] Je pensais que c'était une société où on pouvait avoir une carrière professionnelle, où on pouvait s'épanouir, où on pouvait, en rapport du travail fourni et de ses compétences, avoir un salaire adapté.» (Salarié protégé, délégué syndical, cadre, 42 ans, Bac, PME, demande de licenciement pour faute)
}

En effet, les salariés trouvent d'importants avantages à cet échange. L'autonomie et la responsabilité constituent une partie du paiement de la contribution des salariés. Or ce type de rétribution est assez particulier : plus les salariés s'engagent et plus ils acquièrent d'autonomie et de responsabilité. On est là dans le domaine des ressources qui ne s'usent pas quand on s'en sert, bien au contraire ainsi qu'a pu le mettre en lumière Hirschman (1986). De ce fait, la négociation autour de la contribution et de la rétribution perd, en partie, son sens puisque le coût de l'activité et le bénéfice sont confondus. En outre, en plus du plaisir qu'on peut en retirer de l'activité, s'investir dans ce type d'action pour d'autres raisons que la rétribution, c'est 
aussi acquérir l'impression de devenir "une personne" comme le décrit Hirschman quand il parle de l'investissement dans une action non-utilitaire.

Le point de vue de l'entreprise en constitue une illustration, venant confirmer le jugement de valeur que ces salariés ont d'eux-mêmes.

\footnotetext{
"Il était très brillant en informatique, mais presque trop pour nous. [...] C'est un type, en 5 ans, il s'est rendu indispensable. [...] C'était un petit génie. C'est pareil, là il faut dire les choses clairement et si vous appelez mon président, il vous le dira." (Directeur d'établissement, PME)

"Le directeur ne tarissait pas d'éloges à mon encontre, donc c'était bien." (Salarié protégé, membre comité d'entreprise, délégué du personnel, non-syndiqué, 29 ans, cadre, Bac+2, PME, demande de licenciement économique)
}

Ces salariés arrivent ainsi, à partir d'un investissement fort dans leur activité professionnelle, (voir plus loin) à transformer, en partie, la situation de subordination inhérente aux relations salariales en situation de dépendance (Correia, 2003) ce qu'Albert Memmi (1979), distingue radicalement. "Si le dépendant et le dominé (utilisé dans le même sens que subordonné par Memmi) sont tous les deux aliénés, ils ne le sont pas de la même manière [...] : le dépendant consent plus ou moins à son aliénation, le dominé, non. La raison en est claire : le dépendant trouve son profit à l'être ; le dominé non. On peut certes, être à la fois dépendant et dominé, mais cette coüncidence n'est ni automatique ni nécessaire." En outre, on peut ajouter à cette distinction le fait que la subordination est un concept statique car elle est à sens unique, alors que la dépendance est par nature dynamique puisqu'elle est réciproque : si le salarié ne tient pas ses engagements, cela a des effets directs sur le travail de son supérieur et probablement aussi des effets plus discrets sur la carrière. Or pour passer du lien de subordination à une situation de dépendance, il faut que les relations entre supérieurs et subordonnés, les relations d'autorité, se modifient, en y intégrant la confiance. En effet, autorité et confiance ne s'opposent pas. D'après Boudon et Bourricaud (1986), la confiance est liée à l'autorité : "on parle de l'autorité d'une personne, d'une institution, pour signifier qu'on leur fait confiance, qu'on accueille leur avis, leur injonction, avec respect, faveur, (...) et qu'on est disposé à y déférer " On a ainsi des relations entre salariés et subordonnées qui ne sont pas totalement prescrites par le contrat de travail et qui diminuent très fortement le poids de la subordination.

En outre, ces jeunes salariés n'ont pas subi l'imprégnation politique, dans son sens culturel, qu'a subi la génération antérieure, ce qui les éloigne d'autant des organisations syndicales et des engagements militants.

\section{4) Les effets des candidatures individuelles}

Signalons tout d'abord que ce type de positionnement ne conduit pas nécessairement à une grande implication dans le mandat, et ce, pour deux raisons : parce que la référence au collectif est assez faible mais aussi parce que les salariés ont plutôt tendance à s'adresser, pour leurs revendications, à des militants plus qu'à des élus non-syndiqués.

\footnotetext{
"(Qu'est ce que vous avez fait quand vous étiez DP ?) Pas grand-chose, sincèrement pas grandchose. [...] Parce que ce n'est pas vers moi que les gens venaient au départ, ils venaient plutôt vers une des secrétaires de direction. Les gens savaient qu'elle faisait partie du syndicat.» (Salariée protégée, délégué du personnel, membre du CHSCT, non-syndiquée, 33 ans, CAP, agent de maîtrise, PME, demande de licenciement économique)
} 


\section{La valorisation de l'exemplarité personnelle}

La non-syndicalisation conduit à des candidatures dont la fonction va être, pour reprendre l'expression de Goffman (1973), "la présentation de soi" plus que la présentation d'un collectif structuré dont le salarié serait le représentant. Les relations des élus non-syndiqués au collectif sont, de ce fait, ambiguës : ils se présentent, affirment-ils pour la plupart, afin de remplir des missions d'intérêt général qui dépassent leur seul intérêt, mais en même temps le collectif organisé que représente le syndicat fonctionne comme un repoussoir.

\footnotetext{
"En tant qu'indépendant, je n'avais pas du tout l'âme syndicaliste, ça c'est clair,.[...] (Même l'inverse ?) Oui, à une époque oui." (Salarié protégé, délégué syndical, cadre, 42 ans, Bac, PME, demande de licenciement pour faute)
}

Ils semblent ainsi porteurs de leur seule individualité, qu'ils conçoivent comme exemplaire ou, au minimum, comme plus exemplaire que celle des salariés protégés encartés dans une organisation. La présentation de soi étant dotée d'une composante narcissique explique que les individus peuvent concevoir d'être en quelque sorte plus exemplaires que leurs collègues. Mais il ne s'agit pas seulement de caractéristiques de "personnalité" dont sont dotés les représentants non-syndiqués, cette tendance va de pair avec le fait de détenir des ressources cognitives. Or ces ressources cognitives, toutes choses égales par ailleurs, sont étroitement articulées au niveau de diplôme détenu et à la catégorie socioprofessionnelle.

Les catégories socioprofessionnelles les plus hautes ont plutôt tendance à croire qu'elles peuvent régler elles-mêmes leurs problèmes sans avoir besoin de passer par une régulation collective ${ }^{1}$. Cette tendance est aussi plus ou moins facilitée par le type de fonctions détenues : à l'inverse des délégués syndicaux, désignés par une organisation et donc très fortement marqués par un collectif, les fonctions de délégué du personnel ou d'élu du comité d'entreprise peuvent sembler plus dépendantes de l'exemplarité personnelle ou de compétences relationnelles. C'est ce qui explique, pour partie, des clivages de positions entre les délégués syndicaux, qui ont plutôt tendance à s'opposer par principe à tout ce qui tire vers l'individualisation, et les autres représentants des salariés qui acceptent bien mieux des dispositifs allant dans ce sens ${ }^{2}$.

Le mandat occupé selon la catégorie socioprofessionnelle éclaire également ces différenciations : les ouvriers occupent plus souvent les positions de délégué syndical $(33,6 \%$ des délégués syndicaux sont ouvriers contre 10,2\% qui sont cadres), alors que les fonctions d'élu au comité d'entreprise et de délégué du personnel sont plus souvent occupées par des agents de maîtrise $(23,2 \%$ des élus au comité d'entreprise) et des employés $(32,6 \%$ des délégués du personnel) ${ }^{3}$.

\footnotetext{
${ }^{1}$ L'enquête Réponses constate par exemple que, en tendance, les cadres ont de moins en moins tendance à s'adresser à leurs représentants et les auteurs de l'analyse parlent même de crise de représentation au niveau des cadres. (Furjot D. (2002), De la participation au conflit. Enquête "Réponse" 1998. Questionnaire "représentants du personnel", Document de travail n64, Déc. 2002, DARES.)

${ }^{2}$ Furjot D. (2002), De la participation au conflit. Enquête "réponse" 1998. Questionnaire "représentants du personnel", Document de travail ${ }^{\circ} 64$, Déc. 2002, DARES.

${ }^{3}$ Ibid.
} 


\section{L'importation du monde domestique dans la sphère du travail}

Par l'effet de la personnalisation du mandat mais aussi par le fait de considérer l'entreprise comme une référence identitaire, les relations interindividuelles ne se cantonnent pas à l'échange salarial.

C'est en effet le monde domestique décrit par Boltanski et Thévenot, où les êtres sont qualifiés par la relation qu'ils entretiennent avec leurs semblables et où les relations sont "désintéressées", qui se révèle ici.

"Il était assez proche du président (et son) père [...] est un ami de notre président." (Directeur d'établissement, PME)

Cette forme domestique est mise en oeuvre "dans un mode de gestion du personnel qui valorise l'expérience spécifique acquise par l'ancienneté dans la maison". Les relations dans l'entreprise rassemblent alors, par bien des côtés, à celles que l'on peut avoir dans une famille.

"Puis j'aimais bien à la base le fait que c'était familial, parce que comme j'étais indépendant, j'avais un contact direct avec le PDG, donc ça, ça me plaisait bien, j'avais de très bons rapports d'ailleurs avec [le Dg]." (Salarié protégé, délégué syndical, cadre, 42 ans, Bac, PME, demande de licenciement pour faute)

Dans ce mode de relation à l'entreprise, c'est exclusivement le jugement d'un chef qui fait sortir les salariés du rang. Les relations se vivent alors sur le versant du charisme.

\footnotetext{
"Moi quand je l'ai rencontré la $1^{\text {ère }}$ fois, j'ai été sous le charme, mais c'est un sacré monsieur dans les affaires".» (Salarié protégé, délégué syndical, cadre, 42 ans, Bac, PME, demande de licenciement pour faute)
}

De ce fait, l'investissement dans le travail dépasse les obligations contractuelles, comme dans le cas de ce salarié qui rappelle tout au long de l'entretien à quel point il a pu se dévouer pour l'entreprise.

"J'ai beaucoup travaillé, j'attaquais début décembre jusqu'à fin avril, c'était tous les jours. Je n'avais
pas de congés, j'ai quand même beaucoup donné. [...] C'est simple, pour les vacances de Noël, je
commençais à travailler à 8 heures du matin, je finissais à 10 heures du soir, il y a même certaines
fois où je terminais à 4 heures du matin pour faire les payes. [...] Mon boulot, c'était tout le temps,
parfois je me réveillais la nuit en me disant : tiens, il faut que je fasse ça. C'était quand même une
implication assez forte." (Salarié protégé, membre comité d'entreprise, délégué du personnel, non-
syndiqué, 29 ans, cadre, Bac+2, PME, demande de licenciement économique)

Cet investissement est souvent partagé par la cellule familiale. Le travail envahit alors tout l'espace du salarié. La meilleure illustration en est donnée par le salarié qui s'est présenté à l'entretien avec sa femme qui est intervenue assez fréquemment pour rappeler à quel point l'investissement pour le travail a pu être envahissant.

". s'investit énormément dans son travail, il travaillait tout le temps. Quand il dit que l'été, ça a été plus calme, en fait, ça a été vrai les 2 premières années, il a eu des week-ends en plus sinon il travaillait tout le temps [...] Ça a été un problème entre nous [...] Parce qu'on ne se voyait jamais. [...] Ça me pesait, au départ on est de L., moi j'ai tout laissé, mes études et tout pour qu'on soit relativement proche, et en fait, il travaillait tellement tout le temps qu'en fait il n'était pas plus proche que quand j'étais à L. [...] J'aurais aimé que de temps en temps, il pense à rentrer à 7 heures le soir, ou qu'il pense à dire "je rentre à midi". [...] On l'a vécu à 2, le travail de J. prenait tellement de place dans notre vie que forcément, son licenciement, on l'a vécu aussi." (Salarié protégé, 
membre comité d'entreprise, délégué du personnel, non-syndiqué, 29 ans, cadre, Bac+2, PME, demande de licenciement économique)

"Pendant 1 an et demi, ça a été beaucoup d'investissements [...] J'ai beaucoup travaillé pour trouver une meilleure implantation pour le produit. Je me suis investi comme un malade pendant 1 an et demi.» (Salarié protégé, membre du comité d'entreprise, délégué du personnel, syndiqué, 37 ans, cadre, Bac+2, PME, demande de licenciement pour faute)

\title{
5) La figure intermédiaire : le passeur à mi-chemin de l'opposition et de l'adhésion
}

Il apparaît important de rappeler que nous décrivons des idéaux types ; les comportements des salariés sont plus souvent des mixtes. Il est même possible de repérer quelques salariés dont les comportements relèvent à la fois de l'opposition et de l'adhésion. La position du salarié protégé est, dans ce cas-là, celle du médiateur, de personnes de consensus qui lient des positions antagonistes, de "passeur", selon l'expression de Michel Serres (1991).

\footnotetext{
"Je pense qu'on était des bons agents de liaison, par rapport à la direction qui arrivait à nous parler sans qu'on leur rentre dedans, et les gens qui étaient nos collègues, le courant passait des 2 côtés." (Déléguée syndicale, non-syndiquée, 47 ans, ouvrière, sans diplôme, PME, demande de licenciement économique)
}

Certains salariés protégés ont en effet pour principe de ni s'opposer, ni s'intégrer et, en même temps, de s'opposer et de s'intégrer.

\begin{abstract}
"Que ce n'est pas parce qu'on est délégué qu'on rentre forcément en conflits partout. Je pense que les choses peuvent se passer intelligemment, de façon équilibrée et pour tout le monde, donc je pense que c'est important de donner une image différente. Mais à côté de ça il y a des choses qu'on peut faire appliquer: c'est la légalité, et il n'y a pas de raisons que les gens soient lésés parce qu'un employeur a décidé que lui, il ne voulait pas le faire, par exemple. Donc ça c'est aussi pour appuyer les droits des salariés et quand même me battre pour eux, parce que je suis quand même de leur côté, je ne suis pas du côté de la direction. Même si je ne veux pas de conflits, il ne faut pas non plus que la direction ait décidé de faire tout et n'importe quoi. Dans un climat le plus possible relationnel, que tout se passe bien, c'est parfait, mais d'un autre côté, il faut aussi que les choses se passent de façon tout à fait légale et je me dois de défendre la salariée en cas de problèmes, qu'ils soient graves ou moins graves." (Salariée protégée, déléguée du personnel, nonsyndiquée, 55 ans, profession intermédiaire, sans diplôme, TPE, demande de licenciement économique)
\end{abstract}

En fait, ils se présentent comme des interfaces équidistantes de ces deux figures qui font le lien entre des intérêts opposés.

\footnotetext{
"Je suis assez sociale en général, je sais que la plupart des gens ont beaucoup d'idées mais n'osent pas forcément les dire, les discussions avec la direction, ce n'est pas toujours facile à aborder pour certaines personnes, donc ça crée plutôt des climats en général. Donc, je pense que je l'ai fait comme ça, je me suis dit pourquoi pas me présenter, mais je pense qu'il n’y a rien qui a motivé si ce n'est mon côté social. Et de ne surtout pas rentrer en conflit, où que ce soit, parce que d'une part avec mon poste d'assistante de direction, je ne pouvais pas me permettre de rentrer en conflit avec la direction, ni même avec les salariés, et donc je pense que je suis plutôt quelqu'un qui essaie de gérer les choses en bonne entente et faire passer les messages qu'il faut quand il faut, aussi bien dans un sens que dans l'autre. [...] Je parle plutôt d'un compromis. [...] Je pense qu'ils ont vu que je n'étais pas quelqu'un qui cherchait, comme un syndicat qui cherche vraiment à rentrer en conflit. Je pense qu'ils ont vu que j'étais une personne sensée et qui essayait de faire les choses au mieux.» (Salariée protégée, déléguée du personnel, non-syndiquée, 55 ans, profession intermédiaire, sans diplôme, TPE, demande de licenciement économique)
} 
Comme l'indique clairement la salariée citée ci-dessus, ces passeurs conçoivent leur rôle de représentant du personnel comme tout à fait particulier et opposé à la logique syndicale. De ce fait, leur choix de se présenter aux élections ne s'inscrit pas dans une stratégie d'affrontement. Pour eux, leur personnalité les destine tout naturellement à occuper cette fonction. La légitimité de la fonction occupée s'appuie beaucoup plus sur des qualités particulières qu'ils détiennent que sur les définitions juridiques liées à la situation de subordination et à la volonté du législateur de réduire le déséquilibre inhérent à la situation de subordination. Tout naturellement, la confiance dont ils se prévalent se fonde sur la qualité de leurs relations interindividuelles. Le mandat qu'ils occupent n'est alors qu'une enveloppe pratique pour jouer leur rôle de passeur ou de traducteur.

\begin{abstract}
"Je pense que je passais plus auprès des gens, je pense que le côté que j'ai d'être assez proche des gens et de ne pas vouloir rentrer en conflit. Les gens me connaissaient depuis longtemps et je pense que je passais bien à tous points de vue par rapport à eux, c'est-à-dire ne pas chercher à semer la zizanie juste pour la semer. Je pense que c'est parti sur un climat de confiance de la part des salariés et d'un relationnel probablement qui faisait que les gens ont adhéré à ma candidature.» (Salariée protégée, déléguée du personnel, non-syndiquée, 55 ans, profession intermédiaire, sans diplôme, TPE, demande de licenciement économique)
\end{abstract}

\title{
III- L'influence des stratégies des entreprises
}

Jusqu'ici, nous avons analysé les différentes manières d'occuper le mandat en fonction des orientations personnelles des représentants du personnel, tout en les référant à des modifications sociales d'ampleur qui ont modifié en profondeur le rapport des individus aux collectifs. Toutefois, parce que ces différences sont aussi le produit des interrelations entre représentants du personnel et représentants de l'entreprise, il nous faut comprendre comment ce dernier acteur influe sur le comportement des salariés protégés.

Avant d'analyser cette influence, il nous faut situer les limites de notre travail. Quand nous avons réalisé nos entretiens, malgré nos précautions, nous avons souvent été perçus comme des agents mandatés par le Ministère du travail et donc placés dans une posture de contrôle, éventuellement dotés de possibilités de sanction.

De ce fait, les représentants des entreprises ont développé une argumentation stratégique qui présentait la demande de licenciement des salariés protégés comme le produit de la fatalité ou du comportement inadéquat des représentants du personnel. Même s'ils étaient viscéralement opposés à l'existence des syndicats, aucun de nos interlocuteurs n'aurait pris le risque de défendre ouvertement cette thèse.

De manière identique, les salariés protégés, surtout quand leur situation professionnelle était compliquée, tendaient, et pour les mêmes raisons, à élaborer une argumentation inverse, rejetant la responsabilité sur l'entreprise soupçonnée d'avoir des visées antisyndicales ${ }^{1}$.

\footnotetext{
${ }^{1}$ Par exemple ce représentant du personnel qui décrit une opération d'éradication des oppositions et un acharnement sur le personnel et dont l'épouse continue à faire partie du personnel de l'entreprise sans avoir de problèmes particuliers.
} 
La vérification de la réalité des thèses présentées s'est en partie effectuée par l'interrogation d'autres acteurs : inspecteurs du travail et élus de l'entreprise. Toutefois, l'inspecteur du travail ne peut la plupart du temps que faire des suppositions sur la réalité de ce qui lui est présenté. L'opinion des autres élus est également très orientée. Quand ces élus font partie d'une organisation syndicale, ils font par principe rarement porter la responsabilité des difficultés sur les épaules des salariés. Leur soutien au salarié mis en cause est plutôt la règle. A l'inverse, les élus non-syndiqués peuvent être soupçonnés d'être manipulés par leur hiérarchie, d'autant plus que certains de ceux que nous avons interrogés ont été élus en lieu et place des représentants du personnel dont le licenciement est demandé. Parfois, ces nouveaux élus prônent un positionnement apolitique ou clairement antisyndical, visible par exemple dans l'entretien ci-dessous où la critique du comportement des représentants du personnel, qui sont décrits comme militants de la CGT alors qu'ils sont à la CGC, se termine par le rejet de tout positionnement syndical.

\footnotetext{
"Je me suis présenté afin qu'on puisse renouveler le dialogue social entre la direction et l'ensemble des salariés de l'entreprise puisqu'il n'y avait plus de dialogue possible depuis plus d'un an entre les représentations syndicales et la direction. [...] Le seul problème, c'est que je ne souhaite pas adhérer à une organisation syndicale." (Secrétaire CE, non-syndiqué, 47 ans, PME)
}

Nous devons donc rester extrêmement prudents sur l'interprétation que vous faisons des différentes versions qui nous sont présentées. Un travail plus systématique dans chacune des organisations serait nécessaire pour valider les pistes que nous dessinons ici $^{1}$.

Nous pouvons prendre pour point de départ l'affirmation de Maria-Teresa Pignoni et Patrick Zouary "les circonstances et les raisons de l'évolution des licenciements pour motif personnel restent largement méconnues. [...] Sanction d'une faute ou d'une insuffisance professionnelles, démission déguisée ou licenciement négocié... Le licenciement autre qu'économique est difficile à cerner, et la catégorie administrative et statistique "autres licenciement" ne rend pas compte de l'hétérogénéité des situations qui en sont à l'origine" ${ }^{12}$. On peut ajouter que la catégorie licenciement économique peut aussi être interrogée : ce motif est le premier motif de licenciement pour les représentant du personnel alors qu'il est minoritaire pour l'ensemble des salariés ${ }^{3}$. On peut donc donc faire l'hypothèse que certaines entreprises saisissent cette occasion pour tenter de se débarrasser de certains salariés protégés. En outre, les licenciements pour inaptitude (nous le verrons) sont souvent un "habillage" qui permet de sortir d'une situation bloquée.

Tout cela invite à ne pas s'arrêter à une définition administrative du licenciement et à regarder de plus près les stratégies des entreprises et leurs conséquences sur les relations avec leurs représentants du personnel. En effet, c'est bien la configuration relationnelle du salarié protégé et de son entreprise ${ }^{4}$ qui explique les situations pacifiées comme les situations de conflit.

\footnotetext{
${ }^{1}$ Notre projet initial intégrait un travail d'investigation plus approfondi dans chacune des entreprises par le biais d'entretiens avec d'autres membres du personnel. Pour des raisons pratiques, cette méthodologie n'a pu être retenue.

${ }^{2}$ Pignoni M-T., Zouary P. (2003) "Les nouveaux usages du licenciement pour motif personnel", Premières informations, juillet $2003 \mathrm{n}^{\circ} 28.2$.

${ }^{3}$ Le pourcentage de demandes de licenciement de salarié protégé pour motif économique représentait 4,3\% de l'ensemble des licenciements économiques, en hausse depuis la fin des années 1990 (3,5\% en 1999, 2,6\% en 1998 et 1,5\% en 1989). Il faut maintenant diviser ces chiffres par 4 pour connaître le pourcentage relatif de salarié protégé concerné par des demandes de licenciement pour d'autres motifs.

${ }^{4}$ En fait comme l'entreprise est une entité juridique concrètement constituée d'individu, l'accord ou le désaccord ne peut jamais être uniquement institutionnel. : il s'agit toujours d'un mixte entre logiques institutionnelles et rapports interpersonnels. Pour la clarté de l'exposition, nous simplifierons la présentation que nous faisons des logiques industrielles, en réduisant ces rapports à leur unique dimension institutionnelle.
} 


\section{A) "L'industrialisation" de la gestion du personnel intègre les oppositions}

La première configuration est celle d'une grande entreprise et de représentants du personnel qui s'affirment dans la différenciation des positions.

L'opposition peut en effet être mieux tolérée dans une grande entreprise qui peut lisser cette opposition par l'objectivation des fonctions. Mais, plus important encore, c'est le degré de formalisation de l'organisation et de sa gestion du personnel qui est déterminante pour que l'entreprise accepte ce mode d'occupation du mandat. Mintzberg (1990) a, par exemple, montré que les formes de syndicalisation sont très différentes selon que l'on se situe dans une organisation bureaucratique, ce qui chez cet auteur regroupe aussi les organisations industrielles ou dans une structure professionnelle (université, hôpital, entreprise de presse, etc...). Les organisations bureaucratiques sont caractérisées par des taux de syndicalisation généralement élevés : les opérateurs - à savoir tous ceux qui réalisent les missions de base de l'organisation - y sont naturellement conduits à défendre des intérêts communs, étant donné l'étroite codification de leur travail.

Mais c'est l'approche de Boltanski et Thévenot (1991), qui ont importé dans le champ sociologique les apports de l'école économique de la régulation et des conventionnalistes, qui est la plus pertinente pour notre propos ${ }^{1}$.

On peut alors rattacher l'occupation du mandat de manière oppositionnelle et le fait que l'entreprise soit très marquée par le monde industriel, qui est caractérisé par la standardisation, la stabilité temporelle dans l'avenir et l'anonymat des "êtres" fonctionnels. Les relations avec les représentants des salariés s'inscrivent de ce fait dans des procédures normalisées, dans lesquelles la plupart des règles juridiques sont scrupuleusement respectées.

"(L'entreprise) fait toutes les procédures chaque fois qu'elle peut, bien qu'il y ait eu 2 procèsverbaux d'entraves dont un qui a conduit à une condamnation. Ils considèrent qu'ils font le nécessaire, je pense d'ailleurs que par rapport à beaucoup d'autres entreprises, ils font le nécessaire objectivement." (Inspecteur du travail)

Les fonctions représentatives sont alors, pour les dirigeants, un des paramètres à prendre en compte qui est largement indépendant des personnes qui les occupent et des orientations qu'elles défendent.

\footnotetext{
"Comment l'entreprise considère les fonctions représentatives ?) Ce n'est pas les images anciennes qu'on peut avoir, elle le reconnaît au sens premier du terme." (Inspecteur du travail)

"(Les instances représentatives du personnel) ça sert à travailler ensemble, tous les problèmes d'organisation, de formation, d'être transparents, quand ça va, on le dit, quand ça ne va pas on le dit. Et même avec eux, c'est du donnant-donnant. Quand ça va, ils ferment leurs gueules, quand ça
}

\footnotetext{
${ }^{1}$ Pour eux, toute situation est une relation entre états-personnes et états-choses. Il faut, pour pouvoir analyser la situation, que les états-choses aient sens pour les états-personnes qui s'y engagent. Mais "la faculté de se détacher de l'environnement immédiat, de se soustraire à la confusion de ce qui est en présence pour relier les êtres disponibles à un ordre d'importance, constitue la capacité minimale pour s'engager dans des situations sans s'y perdre". Pour les auteurs, "c'est par l'expérience des épreuves que les personnes apprennent à se conduire avec naturel" dans les différents mondes. "Ce sont souvent des situations troubles qui conduisent à des incertitudes de grandeur et exigent, pour être résolues, un recours à l'épreuve". Pour les auteurs, chaque situation est insérée dans un monde parmi six mondes possibles. Chaque monde présente des caractéristiques particulières. Ils peuvent tous être décrits d'après deux grandes variables : Un ordre de légitimité qui décline des ordres de grandeur (ce qui distingue le "petit" du "grand" dans chaque monde) des valeurs centrales ainsi que des relations entre les personnes. En outre, des équipements (outils, procédures) sont spécifiques à chaque monde. Il s'agit d'un ensemble cohérent.
} 
ne va pas, ils me rentrent dans le chou. Et c'est comme ça que ça doit fonctionner, mais avec des vrais problèmes, pas avec des procès d'intention ou des trucs, et ça se passe merveilleusement bien, je n'ai jamais connu une situation de confiance comme celle que je vis ces dernières années." (Directeur général, PME)

En effet, parce que les logiques oppositionnelles et les formes organisationnelles industrielles sont relativement congruentes, la différenciation des positions est en grande partie intégrée dans la gestion du personnel.

Les relations oppositionnelles peuvent ainsi être vivables dans une grande organisation industrielle ou bureaucratique, dans toutes les entreprises dans lesquelles les rapports sont institutionnalisés, ritualisés. Les "empêcheurs de tourner en rond", que peuvent être certains représentants des salariés, sont en fait "neutralisés" par les effets de taille et de structure.

L'affrontement est alors structurel : il se déroule en continu, chacun définissant ses droits et devoirs. La question des frontières de ce qui est exigible de la part de l'entreprise est au centre de l'affrontement. La description que fait d'Iribarne (1989) des relations professionnelles en France et qu'il caractérise comme la logique de l'honneur renvoie à ce type d'affrontements. C'est le même type de relations qui structure les affrontements décrits ci-dessous.

\footnotetext{
"Non, parce qu'on a toujours été en guerre tous les 2. C'est ce qui est le plus étonnant, je n'ai jamais lâché, lui non plus. Il m'a fait des menaces plusieurs fois, j'ai eu l'inspection du travail plusieurs fois et je n'ai jamais lâché, ce n'est pas parce que je suis mieux que les autres. Mais je ne me suis jamais mise en porte-à-faux, je n'ai jamais fait ce qu'il ne fallait pas." (Secrétaire du comité d'entreprise, déléguée syndical, 58 ans, PME)
}

Cette conception des relations sociales est ancienne et relativement conforme aux conceptions de la gestion de la main-d'oeuvre présentes dans les grandes entreprises. Les représentants du personnel y sont des intermédiaires incontournables avec lesquels il faut composer. La gestion du social fait alors partie du quotidien. L'obligation légale est intériorisée et acceptée. Les instances représentatives du personnel remplissent pleinement leur rôle, ainsi qu'en témoigne la responsable des relations sociales d'un grand groupe :

\footnotetext{
"Je suis de l'école où les délégués sont là pour représenter les salariés parce qu'ils croient à une certaine idéologie, quand je vois les délégués se servir les premiers, ça me rend malade. Ça m'a vraiment fait piquer des colères, parce que ce n'est pas pour ça qu'on est délégué, ou alors on fait autre chose.[...] Le rêve d'un DRH, c'est d'avoir des délégués qui sont costauds, qui remplissent bien leur rôle. Un délégué comme M..., qui n'était pas facile, mais avec qui au moins, on sait où on le trouve, une fois qu'on l'a trouvé, on discute. Mais on ne discute pas pour lui, on discute pour ce qu'il représente.» (DRH, grande entreprise)

"Toute l'équipe qui est partie, notamment l'équipe de la CFDT mais aussi de la CGT, c'est une équipe de personnes militantes, donc on savait très bien où on allait les trouver et c'était parfois des empêcheurs, mais ils jouaient bien leur rôle. J'ai toujours eu beaucoup de respect pour ces gens-là, même dans le conflit.» (DRH, grande entreprise)
}

On peut alors résumer cette configuration et ses effets dans un tableau que nous allons graduellement remplir. 


\begin{tabular}{|l|c|l|}
\hline Mode de management & $\begin{array}{c}\text { Industrialisation, } \\
\text { Formalisation des procédures } \\
\text { Mode d'occupation } \\
\text { du mandat }\end{array}$ & Personnalisation du management \\
\hline $\begin{array}{l}\text { Différenciation des } \\
\text { positions, opposition }\end{array}$ & Stabilité des relations & \\
\hline Intégration & & \\
\hline
\end{tabular}

L'occupation du mandat sur un mode oppositionnel repose, de manière assez surprenante, sur des relations pacifiées, à défaut de relations harmonieuses : il y a un relatif accord sur les positions respectives et l'apport des représentants des salariés à l'organisation. Elle est de ce fait, en théorie, dotée d'une grande stabilité. Toutefois cette stabilité n'est assurée que si rien ne change.

Si la santé économique de l'entreprise se dégrade brutalement, les divergences se durcissent et finissent par se transformer en conflit. L'antagonisme antérieur devient plus marqué : les oppositions salariales, qui font partie de l'antagonisme "normal", sont remplacées par des oppositions qui renvoient aux conditions d'existence même des protagonistes (survie de l'entreprise versus préservation de l'emploi des salariés). Toutefois, cet antagonisme peut être tempéré si tous s'accordent sur le statut des contraintes qui président au changement. Tant qu'elles sont considérées comme des contraintes exogènes, les relations restent dans le registre de l'opposition traditionnelle. Puisque ces contraintes rendent le licenciement quasiinéluctable et imposent de traiter les salariés protégés comme les autres, cela a pour effet d'aboutir aux licenciements "consensuels", c'est-à-dire acceptés par l'ensemble des parties.

Si par contre, l'une des parties soupçonne l'autre d'utiliser le changement, le licenciement ou la fermeture de l'établissement ou de l'entreprise pour des stratégies à long terme, le conflit gagne en dureté. Dans un tel contexte, la composante relationnelle apparaît fondamentale dans l'équilibre de la configuration. De ce fait, le changement d'interlocuteur constitue souvent le principal élément déclencheur.

\footnotetext{
"(Le directeur) est arrivé en août 1999, c'est quelqu'un qui est jeune qui a 29 ans, donc il y a 3 ans, il avait 26 ans, il avait les crocs et les griffes qui lui poussaient, donc il était salaud et [...] on a senti que ça ne pouvait pas durer, et les gens venaient nous voir en disant : "il m'a fait ci, il m'a fait ça". (Salarié protégé, délégué syndical, membre CHSCT, syndiqué, ouvrier, 44 ans, CAP, demande de licenciement pour faute)
}

Le nouvel interlocuteur hiérarchique, souvent pour "imposer sa marque", s'astreint à se distinguer de ce qu'ont fait ses prédécesseurs et tire alors le mode de management vers la personnalisation. C'est une des raisons principales de modification de la configuration relationnelle antérieure, non seulement parce qu'elle modifie un des pôles de la configuration mais, surtout, parce qu'elle remet de ce fait aussi en cause l'équilibre antérieur.

"Au départ, je trouvais que (le nouveau directeur) était quelqu'un de sympa, et puis il s'exprimait bien. [...] $2^{\mathrm{e}}$ mise à pied, c'était sous (ce directeur), en 2001, une mise à pied de 3 jours, parce que je harcelais mes collègues de travail. (Salarié protégé, délégué syndical, membre CHSCT, syndiqué, ouvrier, 44 ans, CAP, demande de licenciement pour faute) 
Mais même en l'absence de changement d'interlocuteurs, la répétition d'une situation perçue comme injuste (comme le marque l'utilisation du terme "imbécile", ci-dessous) peut servir de déclencheur.

\begin{abstract}
"On commençait à en avoir un peu ras-le-bol et à ne pas être entendus, à être pris pour des imbéciles à chaque fois qu'on disait quelque chose, on nous disait qu'on avait tort. Et surtout, on avait aucune information, ni sur la société, ni sur les résultats de notre magasin, ni sur rien. [...] On n'a pas 50 moyens de faire bouger les choses, on s'est dit la seule manière, en plus ça tombait bien à ce moment-là parce qu'il y allait avoir des élections du personnel, on s'est dit, on va essayer de se faire élire.» (Salarié protégé, membre du comité d'entreprise, délégué du personnel, syndiqué, 37 ans, cadre, Bac+2, PME, demande de licenciement pour faute)
\end{abstract}

En fait cette forme d'injustice est un peu particulière : elle n'est pas fondée sur le sentiment qu'a le salarié d'être maltraité. Il s'agit d'un sentiment d'injustice qui vise les individus pris un à un, et dont le salarié protégé fait partie.

Pour résumer, on peut dire que plus le lien des salariés protégés à l'entreprise est désincarné, objectivé, plus les changements doivent être importants pour que le conflit se développe. Le lien entre proximité et radicalité du conflit mis en évidence par Simmel, commence ici à être perceptible. Dans un tel contexte, la représentation collective permet d'équilibrer un minimum le rapport de forces. Mais pour des salariés qui s'inscrivaient jusque-là dans des stratégies individuelles, la constitution d'un collectif exige de rencontrer des collègues qui ont à peu près les mêmes problèmes.

\footnotetext{
"Je ne crois pas à ces groupements qui défendraient les salariés. Il y a trop de magouilles dans tout ça pour que ce soit réel, je ne crois pas. [...] Je ne crois pas au collectif, aux gens qui représentent tout ça. Mais moi, à titre individuel, ou (mon collègue) ou d'autres personnes, à titre individuel, peuvent défendre dans une société d'autres personnes." (Salarié protégé, délégué syndical, cadre, 42 ans, Bac, PME, demande de licenciement pour faute)

"Donc les problèmes ont commencé. Là-dessus, j'ai rencontré une ancienne personne des chaussures X qui avait été embauchée là-bas par hasard, et il avait les mêmes problèmes. Donc on a commencé à se battre." (Salarié protégé, délégué syndical, cadre, 42 ans, Bac, PME, demande de licenciement pour faute)
}

Pour s'appuyer sur un collectif qui dépasse les relations de complicité, il faut passer de l'injustice individuelle à une injustice de nature différente (de profession ou de classe) et donc intégrer ses rancoeurs et ses désirs dans un combat plus large. S'apercevoir que le combat personnel ou de quelques-uns ne débouche sur rien peut s'avérer un bon déclencheur pour s'interroger sur l'intérêt de l'adhésion à un syndicat même si celui-ci semble éloigné de ses valeurs.

\footnotetext{
"À la base, (notre PDG est) un marchand de tapis. Par ruse, il arrivait toujours à nous manipuler, en nous promettant ceci ou cela. On arrivait à une discussion, des joutes oratoires, un peu marrantes quelquefois. Mais au bout de 6 mois, on était lassé parce que rien ne venait, il nous promettait, [...] J'ai toujours espéré, parce qu'il était très rusé, il promettait. Je suis quelqu'un de patient à la base, je me disais qu'on allait discuter, dialoguer. [...] À la base, on était un peu naïf, on ne connaissait pas du tout le monde syndical. [...] On n'y allait pas pour se battre ou pour créer un conflit, on y allait parce qu'on était persuadé que K. est une bonne société, mais qu'il y avait un danger quelque part, que c'était un échange, c'est-à-dire qu'on pouvait la faire évoluer. [...] On n'y allait pas pour le conflit, c'est d'ailleurs pour ça qu'on a choisi la CGC. On n'est allé ni à FO ni à la CGT, là aussi on était un peu naïf, on pensait que la CGC était un peu moins virulente que les autres syndicats." (Salarié protégé, membre du comité d'entreprise, délégué du personnel, syndiqué, 37 ans, cadre, Bac+2, PME, demande de licenciement pour faute)
} 
Il n'y a pas de frontières absolues entre les différentes formes de conflit selon la manière d'occuper le mandat. Toutefois, passer de l'injustice individuelle à l'injustice collective a pour effet de généraliser le conflit et, par là même, paradoxalement, de rendre les représentants du personnel moins sensibles aux blessures personnelles. Les militants syndicaux sont, de ce fait, plus protégés des blessures narcissiques que les représentants du personnel non-syndiqués.

Par contre, il est parfois difficile de repérer une situation ou un événement déclenchant auquel on puisse rattacher le conflit. Les modifications peuvent ainsi se référer à des faits apparemment anodins qui peuvent faire naître une dégradation lente des relations qui peut s'analyser comme le passage progressif d'une relation individuelle à un conflit personnalisé. $\mathrm{Ce}$ processus de dégradation trouve généralement son origine dans une succession de malentendus. La manière dont les salariés protégés et les dirigeants rendent compte du début de cette dégradation est assez éclairante.

"Il a fini par demander au PDG s'il ne pouvait pas être transféré [...] au siège, et il a eu une réponse un peu normande, on va réfléchir, etc... [...]. En fait ça a traîné cette histoire. Ensuite, on a décidé pour des raisons un peu stratégiques qui n'ont rien à voir avec ça, de changer complètement le système de vente [...] qu'on a acheté [...]. Ça c'est passé comme ça et finalement de fil en aiguille, ils ne l'ont pas pris au siège. [...] Ce n'était pas un désaveu parce que, lui, ils l'aimaient bien, ce n'est pas le problème. Le problème c'est qu'il y avait un changement de stratégie au siège. En parallèle, d'ailleurs (la meilleure) preuve (en) est qu'on lui a payé une formation lourde de gestionnaire, il a fait une formation payée par l'entreprise qui a coûté une fortune." (Directeur établissement, PME)

"Simplement, moi, je pense qu'il y a eu une succession de malentendus, il était persuadé qu'il allait évoluer au niveau du groupe, dans sa tête. Il est jeune [...], Et dans sa tête, il s'est dit, ils ne feront jamais rien sans moi, enfin il était parti là-dedans. Au niveau du site, ça devenait un peu limité, donc je pense que toute cette histoire, c'était un peu un malentendu." (Directeur établissement, PME)

"En fait j'ai vu le PDG un mardi matin, quand je suis arrivé, on s'est vu, et là, il y a eu une petite prise de bec. En fait, il m'a sermonné, il est parti en claquant la porte, en disant qu'il ne s'attendait pas à ça de moi, que je l'avais déçu, que c'était inadmissible. Enfin, voilà, après il a fait la gueule pendant 3 jours, et après ça c'est tassé, en façade." (Salarié protégé, membre comité d'entreprise, délégué du personnel, non-syndiqué, 29 ans, cadre, Bac+2, PME, demande de licenciement économique) 
On peut alors résumer les conditions de stabilité de cette configuration relationnelle ainsi que son évolution probable dans le tableau ci-dessous.

\begin{tabular}{|l|l|l|}
\hline $\begin{array}{l}\text { Mode d'occupation } \\
\text { du mandat }\end{array}$ & $\begin{array}{c}\text { Industrialisation, } \\
\text { Formalisation des procédures }\end{array}$ & Personnalisation du management \\
\hline $\begin{array}{c}\text { Différenciation } \\
\text { positions, opposition des }\end{array}$ & $\begin{array}{l}\text { Stabilité des relations } \\
\text { Si contexte stable et si } \\
\text { soutien collectif }\end{array}$ & $\begin{array}{l}\text { Réactions antisyndicales } \\
\text { Tentatives de licenciement }\end{array}$ \\
\hline Intégration & $\begin{array}{c}\text { Stabilité des relations dans } \\
\text { le court terme } \\
\text { Risque Insatisfaction sur le } \\
\text { moyen terme }\end{array}$ & \\
\hline
\end{tabular}

\section{B) Le management intégratif ou paternaliste ne supporte pas l'opposition}

Il en va tout autrement dans les organisations plus petites, dont l'archétype est une entreprise créée par sont dirigeant et dont il est encore souvent encore le seul propriétaire. Nous avons déjà, en creux à partir de la description du comportement des salariés protégés qui personnalisent leur mandat, ébauché le management qui y règne habituellement. Un salarié protégé le résume de manière saisissante.

"Je ne vais pas dire que c'est de l'esclavage mais il faut être disponible et surtout bien rentrer dans le moule, il y a un moule et il faut rentrer dedans" (Salarié protégé, membre du comité d'entreprise et du CHSCT, syndiqué, agent de maîtrise, 28 ans, BTS, PME, demande de licenciement pour inaptitude)

En effet, chez les dirigeants, la confusion est souvent faite entre leur individualité et leur entreprise, qui leur apparaît comme un prolongement d'eux-mêmes.

"Le patron de K., de droit divin, était seul manager d'une entreprise qui a très vite augmenté ses effectifs et son activité. Ce qu'il sait faire c'est du paternalisme autoritaire, c'est tout ce qu'il sait faire, comme beaucoup de fondateurs d'entreprises, c'est plutôt familial. [...] C'est une boîte familiale donc il y a plusieurs membres de sa famille qui sont également dans les cadres dirigeants de la société." (Inspecteur du travail)

Cette conception de l'organisation a pour corollaire une restriction importante de l'accès aux informations, et une absence de discussion sur les finalités et les objectifs de l'entreprise.

"Ils étaient déjà tellement défavorables au syndicat [...] C'est surtout (le dirigeant) et c'est surtout
une politique qui est basée sur la non-divulgation d'informations, c'est-à-dire que les salariés ne
doivent pas savoir comment fonctionne l'entreprise, ce n'est pas leur boulot. Donc à partir de ce
moment-là, à partir du moment où en tant que CE on veut connaître parce que ça fait partie de
notre travail de connaitre comment fonctionne l'entreprise, comment est géré la machine pour
pouvoir le diffuser aux salariés dans une certaine mesure, lui ne supporte pas ça, il ne supporte pas

\footnotetext{
${ }^{1}$ Cette configuration n'est pas conflictuelle dans son sens habituel : elle se traduit le plus souvent par la démission ou le désinvestissement. C'est pourquoi nous ne la développerons pas ici. Cette configuration est issue des travaux sur les surdiplômés
} 
qu'on vienne mettre le nez dans ses affaires. Donc des gens comme nous, pour lui, on est vraiment des fouilles merde, il ne supporte pas des mecs comme nous, c'est viscéral." (Salarié protégé, membre du comité d'entreprise, délégué du personnel , syndiqué, 37 ans, cadre, Bac+2, PME, demande de licenciement pour faute)

En effet, toute intervention externe est vécue comme une intrusion intolérable. C'est par exemple le cas d'une responsable de production pour qui l'enquête que nous réalisons ne saurait recueillir que des informations générales et ne pas se mêler du licenciement en cours car elle soupçonne tous ces acteurs externes, comme elle l'affirme en ce qui concerne l'inspecteur du travail, d'avoir des a priori en défaveur de l'entreprise.

"Je ne veux pas parler du licenciement, je ne veux pas revenir sur le licenciement, j'ai été claire làdessus. [...] C'était très clair, je ne voulais même pas qu'on se rencontre parce que je sais que ça va être stérile au final. [...] L'inspecteur du travail, c'est défense à $100 \%$ du salarié, ce n'est absolument pas neutre, c'est certain." (Responsable de production, PME)

Cette crainte de remise en cause des orientations patronales et hiérarchiques s'applique aussi à toute organisation collective et a fortiori syndicale qui pourrait porter la contestation.

"Il y avait une grande peur de la direction par rapport aux syndicats" (Membre du comité d'entreprise, syndiqué, PME)

Cette crainte et ce désaccord prennent leur source dans une conception particulière des relations sociales, qui devrait, d'après eux, rester cantonnée aux œuvres sociales.

"Donc pendant 2 ans, il n'y a eu aucun avancement social dans cette société tout simplement parce que les gens ne venaient pas faire de l'avancement social, ils venaient chercher un conflit permanent." (DRH, PME)

En outre, cette opposition de principe aux syndicats se double d'un rapport particulier aux représentants du personnel qui tentent d'assumer leur mandat. Ceux qui se sont présentés de leur propre initiative sont immédiatement perçus comme des ennemis et traités comme tels, comme l'indiquent les extraits d'entretiens ci-dessous.

"Le lendemain des élections, j'ai été convoqué tout de suite par la direction. Ils m’ont dit : "on ne comprend pas qu'est ce qu'on t'a fait", j'ai répondu "rien mais j'essaie de nouer un dialogue". Ils ont dit "on ne comprend pas, ça marchait bien, on ne comprend pas pourquoi tu as fait ça." (Secrétaire comité d'entreprise, syndiqué, PME)

" Je pense qu'il y a une politique de pression dès qu'on intègre des nouveaux venus dans des postes à fonction de délégués du personnel, il y a des pressions énormes qui tombent dessus. Et il y a une partie du personnel qui craque et qui s'en va tout de suite." (Secrétaire comité d'entreprise, syndiqué, PME)

Le mandat peut alors devenir source de discrimination, comme en témoigne une Salariée protégée.

"Tout ce qui était syndical et comité d'entreprise était complètement écartés de la promotion, mon salaire n'a pas évolué depuis 15 ans. (À cause de votre mandat ?) Oui, et puis ça a été clairement dit dans l'entreprise, le directeur financier a dit : les délégués syndicaux on n'en veut pas. C'était clair dans l'entreprise." (Salariée protégée, juge prud'hommes, syndiquée, 54 ans, agent de maîtrise, CAP, PME, demande de licenciement pour inaptitude)

Le mandat peut aussi être constitutif, pour l'employeur, d'une trahison et ce, d'autant plus si le salarié se syndique ou bien révèle au grand jour son appartenance syndicale, faisant ainsi du 
même coup entrer le syndicat dans la "maison". Cela se vérifie tout particulièrement dans les petites entreprises où l'employeur et les salariés entretiennent des relations de proximité. Cela se vérifie aussi dans la plupart des cas où les représentants du personnel ont été au départ pressentis pour postuler à cette fonction par leur employeur ou par leur hiérarchie et qu'ils décident de s'affranchir de cette tutelle.

Il faut, pour le comprendre, revenir sur les liens qui unissent certains salariés protégés à leur entreprise. La tonalité générale est celle de la fusion affective. L'entreprise, dans son ensemble, est alors une instance identitaire, une "communauté de destin". Rappelons aussi qu'un certain nombre d'entre eux se sont présentés aux élections sur les recommandations de leur hiérarchie, renforçant l'idée d'une relation fusionnelle. Or ce type de relations s'appuie très fortement sur la confiance. Celle-ci se gagne et est réciproquement et mutuellement méritée (Lorenz, 1996).

De ce fait, les réactions peuvent être assez brutales face à des interlocuteurs qui se positionnent systématiquement sur le respect de leurs droits. Cette revendication, en effet, rompt avec l'affectivité précédente en réduisant les relations à leur seule composante institutionnelle.

\footnotetext{
"Des élus [...] qui avaient une conception au pied de la lettre de leur mandat, ils ont passé beaucoup de temps et d'énergie à apprendre ce à quoi ils avaient droit et ils entendaient se faire respecter de $\mathrm{A}$ à $\mathrm{Z}$ tous leurs droits, le virage a été très dur à prendre pour la direction." (Inspecteur du travail)
}

Paradoxalement, le conflit glisse alors d'un conflit de position à un conflit interpersonnel. C'est pourquoi le désaccord se vit de part et d'autre sur le versant de la trahison. Quand le salarié manifeste son désaccord, ce sont les représentants patronaux qui se sentent trompés.

\footnotetext{
"Des actions de minorités comme (ces salariés protégés), je ne sais pas si vous avez eu connaissance de jusqu'où ont été ce type d'action, parce que les lettres anonymes d'une saleté pas possible.» (DRH, PME)

"Je pense que c'est plus que de la déception, c'est une personne qui à mon sens est une personne aigrie, qui en veut à l'entreprise, en tout cas il en veut à tout le monde patronal (DRH, PME)
}

Signalons que l'argumentation de la hiérarchie nie souvent leur propre incapacité à accepter un autre point de vue dans l'entreprise et renvoie les difficultés rencontrées aux modifications de comportement provoquées par le fait de détenir un mandat.

\footnotetext{
"Avec lui, ça a commencé tout bêtement, à partir du moment où il a été délégué syndical, son comportement a énormément changé. [...] Ca a changé parce que sans être en délégation, ils allaient dans le local $\mathrm{CE}$, le fait qu'ils fumaient là-bas, enfin différentes choses comme ça qu'ils ne faisaient pas avant." (Responsable de production, PME)
}

Quand c'est l'entreprise qui manifeste des divergences, c'est le salarié qui se sent "floué".

\footnotetext{
"Je savais qu'un jour, le mal qu'on me faisait, j'allais leur en mettre plein la tête." (Salarié protégé, délégué syndical, membre du comité d'entreprise, 55 ans, cadre, BEPC, PME, demande de licenciement pour inaptitude)
}

De ce fait, le conflit dépasse largement le cadre du travail et envahit parfois la vie familiale dans son ensemble. Le mandat cristallise alors ce «désamour» né entre l'entreprise et le salarié qui peut évoluer en conflit interpersonnel débouchant sur des pratiques que l'on peut 
juridiquement qualifier de harcèlement professionnel. Cela se vérifie tout particulièrement en période de restructurations où les salariés protégés sont plus "exposés" (il est intéressant de noter que, selon la jurisprudence, la perte de confiance de l'employeur envers le salarié ne peut jamais constituer, par elle-même, un motif pouvant servir de base à une autorisation de licenciement ${ }^{1}$ ).

C'est cette connotation passionnelle qui explique la virulence des réactions à l'encontre des représentants du personnel qui ne se soumettent pas.

\begin{abstract}
"(Un ancien cadre de l'entreprise) nous a expliqué un truc, il nous a dit : la société veut tellement votre peau qu'elle est prête à utiliser ses relations pour vous proposer du boulot ailleurs, pour vous virer quoi. Et puis après, une fois la période d'essai terminée : dehors. On est face à des patrons qui, pour moi, font partie des patrons voyous, qui sont prêts à utiliser les moyens qui ne sont pas légaux pour se débarrasser des gens.» (Salarié protégé, membre du comité d'entreprise, délégué du personnel, syndiqué, 37 ans, cadre, Bac+2, PME, demande de licenciement pour faute)
\end{abstract}

En effet, même si les dirigeants le disent rarement formellement, toute critique sur la structuration de l'entreprise, sur sa stratégie... apparaît vite comme une remise en cause personnelle du dirigeant. En outre, la place qu'occupe la composante identitaire du conflit chez certains salariés protégés implique la recherche d'une reconstruction de son image sociale et d'une restitution de sa valeur sociale qui deviennent, dès lors, des impératifs : il faut qu'ils puissent prouver qu'ils disposent bien de compétences élevées et donc d'une valeur sociale. D'où l'insistance sur le fait qu'ils ont raison et leurs supérieurs ont tort. Or se positionner sur ce terrain, c'est affirmer que les dirigeants sont incompétents, ce que, bien sûr, ces derniers ne peuvent entendre.

\begin{abstract}
"Donc des gens qui n'ont pas l'habitude de gérer des grosses sociétés, comme c'est anonyme, je peux le dire, à mon sens n'avaient pas vraiment les compétences. [...] Il est arrivé à un certain niveau d'incompétences, comme ça nous arriverait à tous, et il n'a pas su se remettre en cause pour développer sa société.[...] À mon sens, ils n'avaient pas vraiment les compétences.» (Salarié protégé, membre du comité d'entreprise, délégué du personnel, syndiqué, 37 ans, cadre, Bac+2, PME, demande de licenciement pour faute)
\end{abstract}

Dans ce type d'affrontements, où les positions statutaires des uns et des autres jouent peu car supplantées par des conflits "d'homme à homme" apparaissent très fragiles. Le nombre d'autorisations de licenciement qui s'adressent à des salariés protégés non-syndiqués (59\% en 2000) et surtout le pourcentage d'acceptations de ces licenciements (91\% en 2000 comme en 1999) ${ }^{3}$ constituent un indice de cette fragilité.

Une première explication de cette fragilité tient à l'orientation interpersonnelle du conflit. Les autres salariés peuvent avoir l'impression, parfois largement justifiée, que les salariés protégés vivent une histoire personnelle, sur laquelle le collectif n'a que peu de prise. Les jugements portés par les différents acteurs se révèlent particulièrement convergents sur ce point.

\footnotetext{
${ }^{1}$ CE 21 décembre 2001, alors, RJS 3/02, n 304

${ }^{2}$ Est ce un hasard, si dans les cas rencontrés, les femmes adoptent, plus que les hommes des positions de compromis ? Il faudrait pouvoir valider cette hypothèse au niveau statistique, mais il semble bien (ou il semblait bien jusqu'à une période récente) que les femmes ne développaient tout à fait les mêmes enjeux sociaux, (Enriquez, 1983 et 1991) ce qui leur permettait de ne pas se retrouver engluées dans un conflit où ce qui est recherché est la défaite totale de l'adversaire.

${ }^{3}$ Merlier R., "Les licenciements de représentants du personnel en 2000", Premières informations, novembre 2002, n 48.2
} 
"Les autres salariés, il n'y a pas eu de réaction du tout, ils ont tous dit c'est son affaire, il fait sa vie. Je ne pense pas qu'il y ait eu de réactions." (Directeur établissement, PME)

"Lui ne nous donnait pas toutes les informations et nous non plus. [...] Il a négocié son départ directement avec l'employeur, sans me solliciter, en tant que membre du CE ainsi que mes collègues (Secrétaire comité d'entreprise, syndiqué, PME)

Une deuxième explication trouve sa source dans la position de force des représentants de l'employeur du fait du lien de subordination des salariés mais aussi du plus grand prestige social dont ils peuvent faire preuve.

"C'était manifestement un peu la chasse aux sorcières. [...] Les salariés se plaignent à longueur d'année. [...] C'est conflictuel en permanence et très violent. [...] Elle vous écrase si vous avez un doute sur vos droits, sur vos pouvoirs, sur vos moyens, elle va en profiter, elle va vous écraser, et donc c'est un peu ce qui se passe au niveau des élus." (Inspecteur du travail)

Dans ce cas, même si des moments d'accalmie surviennent, les relations sont par nature conflictuelles et la disparition des représentants du personnel, empêcheurs de tourner en rond, constitue un horizon permanent de la gestion du social ou au moins une espérance caressée par les dirigeants de l'entreprise.

Une des manières de parvenir à ces objectifs consiste à susciter des candidatures "spontanées". C'est pourquoi l'entreprise se félicite souvent de l'arrivée de nouveaux interlocuteurs.

"Vous avez une liste indépendante de salariés qui voulaient arrêter les dégâts. Quand je dis arrêter les dégâts, c'est le combat permanent, les insultes permanentes qui étaient écrites, il fallait voir, je pense que vous avez un dossier assez clair. [...] Et moi je dis que c'est extrêmement fort et adulte de la part de nos salariés." (DRH, PME)

En effet, les dirigeants espèrent que ces derniers joueront un rôle plus en accord avec leurs attentes.

" C'est-à-dire que les syndicats se sont braqué sur leur position qui avait plus une démarche de demande agressive et non construite qu'une démarche de progrès et de travail avec la direction, ce qui fait qu'il n'y a eu aucune avancée particulière pour les salariés depuis 2 ans. [...] Je pense que le rôle du comité d'entreprise aujourd'hui est de contribuer à développer une culture d'entreprise, plus qu'à faire des chèques cadeaux [...] Je pense qu'avant toute chose, la vocation du comité d'entreprise, c'est de travailler avec la direction pour pouvoir avancer et que tous les salariés puissent avoir un équilibre professionnel qui soit exemplaire vis-à-vis des autres. [...] Quand on commence à écrire, à insulter notre patron, l'entreprise, on insulte son outil de travail, je ne pense pas que ce soit progressif.» (Secrétaire du comité d'entreprise, non-syndiqué, 47 ans, PME)

On peut alors compléter notre tableau en constatant la grande instabilité créée par le croisement de deux tendances à personnalisation conjointe du management et des représentants du personnel. 


\begin{tabular}{|l|c|c|}
\hline $\begin{array}{l}\text { Mode d'occupation } \\
\text { du mandat }\end{array}$ & $\begin{array}{c}\text { Industrialisation, } \\
\text { Formalisation des procédures }\end{array}$ & Personnalisation du management \\
\hline $\begin{array}{c}\text { Différenciation } \\
\text { positions, opposition des }\end{array}$ & $\begin{array}{c}\text { Stabilité des relations } \\
\text { Si contexte stable et si soutien } \\
\text { collectif }\end{array}$ & $\begin{array}{c}\text { Réactions antisyndicales } \\
\text { Tentatives de licenciement }\end{array}$ \\
\hline Intégration & $\begin{array}{c}\text { Stabilité des relations dans le } \\
\text { court terme } \\
\text { Risque Insatisfaction sur le } \\
\text { moyen terme }\end{array}$ & $\begin{array}{c}\text { Très forts risques de conflits sur } \\
\text { le moyen terme }\end{array}$ \\
\hline
\end{tabular}

\section{C) Les configurations relationnelles définissent des enjeux différenciés et exigent des ressources particulières}

Il est possible de résumer le conflit par le type d'enjeux poursuivis que nous présentons dans un tableau synthétique.

\begin{tabular}{|l|l|l|}
\hline Niveau d'adhésion à l'entreprise & Enjeux du conflit & Coûts personnels du conflit \\
\hline Faible & $\begin{array}{l}\text { Droits liés aux positions et aux } \\
\text { statuts }\end{array}$ & $\begin{array}{l}\text { Coûts faibles mais tendance à la } \\
\text { personnalisation du conflit dans la } \\
\text { durée }\end{array}$ \\
\hline Fort & $\begin{array}{l}\text { Identité professionnelle et sociale de } \\
\text { l'individu }\end{array}$ & Coûts psychologiques importants \\
\hline
\end{tabular}

Si les individus arrivent à maintenir le conflit dans les limites d'un conflit de positions, leur valeur sociale ou leur état psychique est relativement protégé.

Par contre, un conflit de longue durée a plutôt tendance à glisser sur le volet de la personnalité. Le basculement vers une problématique de préservation ou de reconstruction d'une identité professionnelle ou d'une valeur sociale impose aux individus des coûts psychologiques beaucoup plus importants que le conflit de positions.

La question de l'usage du droit peut alors être différenciée suivant le niveau d'adhésion de l'individu à l'entreprise et de l'existence ou non d'un soutien de la part du collectif de travail.

\footnotetext{
${ }^{1}$ L'articulation « volonté intégrative » des salariés et « industrialisation de l'organisation » est une configuration stable. Mais ce n'est le cas que sous réserve que le degré de bureaucratisation n'interdise pas toute initiative en normalisant la totalité des comportements des salariés. Il est probable que les salariés quittent l'entreprise assez vite si tel est le cas ou qu'ils adaptent leur comportement en conséquence (exit ou retrait [exit intérieur]). Dans les autres cas, la difficulté tient à ce que la recherche de valorisation des salariés ne puisse se traduire concrètement que difficilement, car les règles d'avancement, par exemple, sont fortement formalisées. L'insatisfaction alors va quasi mécaniquement modifier le comportement des salariés qui peuvent tenter de trouver une voie de sortie négociée.
} 


\begin{tabular}{|l|c|cc|}
\hline Soutien du collectif de travail & Fort & \multicolumn{2}{|c|}{ Faible } \\
\hline Niveau d'adhésion à l'entreprise & $\begin{array}{l}\text { Utilisation de ressources juridiques } \\
\text { en complément du soutien du } \\
\text { collectif }\end{array}$ & $\begin{array}{c}\text { Juridicisation des relations en } \\
\text { remplacement du soutien du } \\
\text { collectif }\end{array}$ & $\begin{array}{c}\text { Utilisation de ressources juridiques } \\
\text { pour un usage individuel }\end{array}$ \\
\hline Fort & $\begin{array}{c}\text { xxxxxxxxxxxxxxxxxxx } \\
\text { Configuration incohérente }\end{array}$ & . \\
\hline
\end{tabular}

Dans l'affrontement, les protagonistes ne sont pas à parité : ce sont les salariés qui se trouvent, le plus souvent, en position d'infériorité. C'est donc en situation d'inégalité que des ressources juridiques peuvent être mobilisées.

Lorsque l'individu est placé dans une configuration où le conflit est personnalisé, le droit constitue une ressource purement individuelle dont l'individu peut se servir de manière tactique. Il peut menacer ses adversaires de rétorsion (par exemple un procès pour harcèlement sexuel ou harcèlement moral) ou de bloquer la situation en jouant sur la longueur de la procédure.

Quand le salarié protégé cantonne le conflit à une différence de positions, le droit devient une arme pour faire respecter les droits et les devoirs inhérents à la position. En se formant par eux-mêmes ou dans l'organisation syndicale, certains salariés manient avec efficacité l'arme juridique. Toutefois, tant que cet usage vient en complément du soutien collectif ou est contrôlé par le collectif, les enjeux des relations sociales restent présents.

Par contre, quand les salariés protégés se laissent entraîner dans des escarmouches juridiques ou lorsque la procédure devient la seule chose importante, un écart se creuse avec le collectif et le droit devient un palliatif au rapport de forces, ce qui peut, sur le moyen terme, se révéler problématique. 


\section{Conclusions intermédiaires : la tendance à la banalisation et à la subjectivation du mandat}

Les différentes modalités d'occupation du mandat que nous avons décrites ainsi que leurs déterminants donnent l'impression que des évolutions de fond qui se déroulent rendent l'occupation du mandat profondément différente de celle qui prévalait à l'époque où les différentes règles juridiques ont été élaborées, c'est-à-dire à partir des années $70^{1}$.

N'assiste-t-on pas à une banalisation du statut juridique et social du salarié protégé sous la pression, notamment, de l'important contentieux sur les rattrapages de salaires qui a eu lieu ces dernières années, et dont le plus significatif demeure celui qui a opposé les salariés protégés de Peugeot à leur direction. Ce contentieux s'est appuyé sur deux arrêts de la Chambre sociale de la Cour de cassation rendus le même jour, Fluchère et Seillier, qui, conformément au droit communautaire ${ }^{2}$, introduisent dans le droit français, un renversement de la charge de la preuve au niveau des discriminations tant syndicales ${ }^{3}$ que sexistes ${ }^{4}$. Le salarié n'a ainsi plus à faire la preuve de la discrimination dont il est victime mais doit fournir des éléments de fait susceptibles de porter atteinte à l'égalité de traitement. Cela peut se faire par le biais de données statistiques apparemment neutres, mais qui affectent une proportion nettement plus élevée de personnes d'un sexe ou d'un syndicat, par exemple (discrimination indirecte). En mettant en exergue le lien entre mandat et conditions de travail, entendues dans le sens large du terme, le droit, et en particulier la jurisprudence de la Cour de cassation, a sans doute contribué à l'alignement des statuts (salariés protégés - non protégés). Cette banalisation des fonctions s'exprime également chez les salariés eux-mêmes qui font état d'un "avant" où la discrimination apparaissait comme un gage d'un "bon syndicalisme" : "Les augmentations de salaires, changer de voiture étaient suspects"; "quand on avait une augmentation, on pouvait passer pour des collabos" ; "il était interdit, pour un élu, d'avoir une promotion". Les discriminations étaient "acceptées" par les militants syndicaux comme faisant partie du mandat. Il existait en quelque sorte une culture du sacrifice qui contribuait aussi à légitimer le mandat. Les représentations sociales attachées au mandat et à son occupation semblent ainsi évoluer.

Ces évolutions ne traduisent-elles pas en même tant qu'elles l'induisent, un recentrage sur la personne ? Ainsi que l'explique F. Duquesne, "Le recentrage des droits fondamentaux sur la personne au travail, à l'initiative du juge social tend à favoriser l'admission, à l'intérieur même de la vie de travail, de séquences où le salarié est autorisé à se soustraire au rapport de subordination" ${ }^{6}$. Le mandat confère une certaine liberté à la personne qui l'occupe. C'est ainsi que la chambre sociale de la Cour de cassation a pu décider qu'un salarié trésorier du CE qui avait détourné des fonds et avait été licencié pour ce fait ne pouvait se voir licencié pour faute

\footnotetext{
${ }^{1}$ Date des arrêts Perrier, Cass. Chambre mixte 21 juin 1974, Perrier, Dalloz 1974, p. 593, Concl. Touffait, p. 596 : «attendu que les dispositions législatives soumettant a l'assentiment préalable du comité d'entreprise ou à la décision conforme de l'inspecteur du travail le licenciement des salaries légalement investis de fonctions représentatives, ont institué, au profit de tels salaries et dans l'intérêt de l'ensemble des travailleurs qu'ils représentent, une protection exceptionnelle et exorbitante du droit commun qui interdit par suite a l'employeur de poursuivre par d'autres moyens la résiliation du contrat de travail ».

${ }^{2}$ Directive de 1997. Aujourd'hui codifiée par la loi du 17 janvier 2002 sur les discriminations à l'article L. 122-45, $4^{\mathrm{e}}$ al. du Code du travail.

${ }^{3}$ Cass. soc. 28 mars 2000, Fluchère, Dick et CDFT cheminots c/ SNCF, Dr. soc. 2000, p. 593, note M.-Th. Lanquetin.

${ }^{4}$ Cass. soc. 23 nov. 1999, Melle G. Seillier c/ CEA, Dr. soc. 2000, p. 593, note M.-Th. Lanquetin.

${ }^{5}$ Paroles de militants CGT, Stage ISST, juin 2003.

${ }^{6}$ Duquesne (F), "Représentants du personnel. Contrat de travail et exercice du mandat", RJS 7/02, p. 603 à 608, p. 607.
} 
grave dès lors qu'"un fait fautif ne peut s'entendre que d'un fait du salarié contraire à ses obligations à l'égard de l'employeur ; qu'en statuant comme elle l'a fait, alors, d'une part, qu'un salarié agissant dans le cadre de ses fonctions de trésorier du comité d'entreprise n'est pas sous la subordination de l'employeur, et alors, d'autre part, que l'arrêt n'a caractérisé aucune faute commise par le salarié dans l'entreprise, la cour d'appel a violé les textes susvisés"1.

Au final, il apparaît parfois difficile de déterminer le rôle effectivement joué par le statut juridique : s'agit-il de protéger le mandat ou la personne? Si le droit se prononce très clairement en faveur de la protection du mandat ${ }^{2}$, l'analyse des pratiques montre l'existence d'une combinaison des deux . Les motifs de l'engagement ressortent de l'intérêt collectif et individuel. C'est ce qu'exprime M. C, DS FO, secrétaire de CE et conseillère prud'homale.

"Il se passait des choses injustes dans les salaires, il fallait mettre le nez dedans et personne n'osait. Mais de fil en aiguille, quand ça m'a atteint personnellement, je me suis dit : il faut faire quelque chose, ça ne peut plus durer." (Salariée protégée, juge prud'hommes, syndiquée, 54 ans, agent de maîtrise, CAP, PME, demande de licenciement pour inaptitude)

Cette banalisation du mandat s'explique, en partie, par la place plus importante occupée par la personne dans la construction de la relation de travail.

Cette personnalisation du mandat trouve son pendant juridique dans la récente consécration, par le Code du travail, du harcèlement moral en tant que catégorie juridique ${ }^{3}$. N'est-on pas en train de glisser d'une logique «d'objectivation du mandat», que le droit a contribué à instituer, à une logique de «subjectivation»? Autrement dit, le mandat serait aujourd'hui intimement lié à la personne qui l'occupe, tant au niveau des représentations qu'en ont les autres salariés et l'employeur, que dans l'exercice du mandat par le salarié protégé, contribuant à "rendre plus acceptable" une séparation qui est aussi plus "douloureuse" ${ }^{4}$.

Plusieurs constats, issus des enseignements dispensés au sein de l'ISST, peuvent être dressés qui contribuent à étayer cette hypothèse :

$1^{\circ}$ Les négociations sur la RTT ont mis en exergue le décalage pouvant exister entre les objectifs du délégué syndical (mettre en place des pauses intégrées dans le temps de travail) et les attentes des salariés (faire reconnaître un temps de pause comme temps de travail mais ne pas l'utiliser afin de raccourcir le temps de présence dans l'entreprise), ce décalage aboutissant à des critiques ouvertes dirigées contre la personne du salarié protégé, critiques pouvant aller jusqu'au désaveu. Les salariés protégés interrogés dans le cadre de notre activité à l'ISST traduisent ces évolutions en termes de montée de l'individualisme : «les salariés sont difficiles à mobiliser»; «les militants viennent voir le syndicat lorsqu'il y a le feu»; «ils nous utilisent»; «autrefois, on était à l'intérieur d'un bonheur collectif». Un inspecteur du travail constate que les modalités d'intervention des inspecteurs du travail se font de plus en plus à partir d'une demande individuelle, alors qu'auparavant, la «clé d'entrée» était un conflit collectif ou un accident du travail.

\footnotetext{
${ }^{1}$ Cass. Soc. 4 juillet 2000, RJS 11/00, $\mathrm{n}^{\circ} 1109$

${ }^{2}$ Lire nos développements antérieurs.

${ }^{3}$ Loi du 17 janvier 2002 dite loi de modernisation sociale »; art. L. 122-49 du Code du travail.

${ }^{4}$ Même si le statut protecteur peut apparaître, pour certains salariés, en particulier ceux qui possèdent un projet professionnel, comme un «statut carcan » qui alourdit la procédure et retarde la séparation.
} 
$2^{\circ}$ Les salariés protégés font état, plus souvent que les autres salariés, de souffrances au travail (environ $14 \%$ des personnes venues consulter la FNATH) ${ }^{1}$, de pressions exercées par l'employeur. Or il semble que les pressions que subissent certains salariés protégés ont glissé depuis le mandat vers le contrat de travail et la personne qui l'occupe. Le conflit porterait donc moins sur les fonctions statutaires que sur la façon dont est réalisé le travail : qualité du travail, manquements professionnels peuvent ainsi être mis en avant par l'employeur, sans qu'il soit aisé de «faire la part des choses».

Ce constat trouverait son prolongement juridique (sans que nous ne disposions de données chiffrées) dans les demandes de licenciement fondées sur une insuffisance professionnelle ou même une inaptitude du salarié protégé. S'agissant de l'inaptitude, l'une des hypothèse (qui reste difficile à prouver) est que la dégradation des conditions d'exercice des mandats syndicaux serait à l'origine d'une dégradation de l'état de santé des personnes qui serait à l'origine de reconnaissances de l'inaptitude débouchant sur un certain nombre de licenciements. Ce n'est alors pas le mandat mais la santé défaillante du salarié protégé qui fonde, par le biais de l'inaptitude reconnue, la demande. Ce glissement permet peut-être d'expliquer, en partie, pourquoi les salariés protégés contestent moins souvent que les employeurs la décision rendue par l'inspecteur du travail.

Cette personnalisation du mandat semble se traduire aujourd'hui par un glissement de la discrimination vers le harcèlement moral, transportant les termes du litiges sur le terrain du conflit interpersonnel tout en générant une certaine opacité quant aux fondements réels du litige.

La jurisprudence s'est forgée, au fil des ans, autour des luttes contre la discrimination des salariés protégés, en s'appuyant sur l'article L. 122-45². La Cour de cassation annule toute mesure discriminatoire touchant la vie professionnelle du salarié protégé, qu'il s'agisse de la rémunération, de l'avancement, de l'accès à la formation ${ }^{3} . .$. . Par conséquent, l'œuvre jurisprudentielle consiste, entre autres, à garantir une égalité de traitement entre les salariés protégés et les autres. Aucune mention du bulletin de paie ne doit ainsi permettre d'établir une distinction entre les heures travaillées et les heures de délégation dont bénéficient les salariés protégés : de telles mentions sont illicites ${ }^{4}$. Le salarié protégé doit bénéficier des mêmes garanties et notamment des recherches de reclassement ${ }^{5}$. Pour ce faire, tant le Conseil d'Etat

\footnotetext{
${ }^{1}$ FNATH, Salariés en détresse : quelles réponses ? L'expérience d'une consultation pluridisciplinaire, document de travail interne, automne 2003, $27 \mathrm{p}$.

${ }^{2}$ Suivant le premier alinéa, «Aucune personne ne peut être écartée d'une procédure de recrutement ou de l'accès à un stage ou à une période de formation en entreprise, aucun salarié ne peut être sanctionné, licencié ou faire l'objet d'une mesure discriminatoire, directe ou indirecte, notamment en matière de rémunération, de formation, de reclassement, d'affectation, de qualification, de classification, de promotion professionnelle, de mutation ou de renouvellement de contrat en raison de son origine, de son sexe, de ses mœurs, de son orientation sexuelle, de son âge, de sa situation de famille, de ses caractéristiques génétiques, de son appartenance ou de sa non-appartenance, vraie ou supposée, à une ethnie, une nation ou une race, de ses opinions politiques, de ses activités syndicales ou mutualistes, de ses convictions religieuses, de son apparence physique, de son patronyme ou, sauf inaptitude constatée par le médecin du travail dans le cadre du titre IV du livre II du présent code, en raison de son état de santé ou de son handicap ».

${ }^{3}$ Cass. Soc. 28 mars 2000, Fluchère, Dick et CFDT Cheminots c/ SNCF, Dr. Soc. 2000, p. 593, note M.-Th. Lanquetin. Le salarié n'a plus à faire la preuve de la discrimination dont il est victime mais doit "fournir les éléments de fait susceptibles de caractériser une atteinte au principe de l'égalité de traitement" ; il incombe alors à l'employeur d'apporter la preuve que cette différence est "justifiée par des éléments objectifs étrangers à toute discrimination".

${ }^{4}$ La Cour de cassation s'est appuyée, dans un raisonnement a contrario, sur l'article R. 143-2 avant-dernier alinéa qui prévoit que "la nature et le montant de la rémunération de l'activité de représentation figurent sur une fiche annexée au bulletin de salaire" ; Cass. Soc. 18 févier 2004, Distribution Casino France c/ Theron, Bull.

${ }^{5}$ CE 30 octobre 1985 - N ${ }^{\circ}$ 40203, Me Garnier, syndic de la liquidation des biens de la S.A.R.L. Engineering et Ouvrages d'Art, Req. ${ }^{\circ} 40203$
} 
que la Cour de cassation procèdent par comparaison ; c'est elle qui permet d'éventuellement faire émerger le lien avec le mandat ${ }^{1}$.

Or la pression s'exerce de plus en plus sur la personne et non sur le mandat, fragilisant celui qui l'occupe, ainsi qu'en témoignent des salariés protégés interrogés :

«On ignorait les gens»; «on était écarté»; «je pouvais rester à mon bureau sans rien faire, ils ne le remarquaient même pas." (Salariée protégée, juge prud'hommes, syndiquée, 54 ans, agent de maîtrise, CAP, PME, demande de licenciement pour inaptitude)

Une autre salariée protégée, secrétaire de CE et syndiquée, expose la nature des pressions subies.

«Mais moi je ne me mets pas en porte-à-faux, jamais. Ce que je ne sais pas faire, je ne le ferai pas. Pendant un moment il voulait me faire faire les fichiers croisés, alors je lui ai dit "je veux bien, mais fais-moi voir", donc il m'a fait voir plusieurs fois. Une fois qu'il a vu que j'ai compris comment ça fonctionnait, il l'a mis dans les mains de quelqu'un d'autre. [...] C'est un combat permanent, je peux vous assurer que je résiste.» (Secrétaire du comité d'entreprise, déléguée syndical, 58 ans, PME)

C'est alors un processus d'éviction du salarié protégé qui est visé par de telles pratiques.

«Là aussi ce sont des "trucs à pas de chance". Lorsque j'ai eu mon accident, on a diagnostiqué une luxation de l'épaule et non pas une fracture. Donc on m'a immobilisé, ma fracture s'est résorbée. En 2000, je ne pouvais plus lever un bras, je ne pouvais plus ni porter un verre ni quoi que ce soit, donc, la fracture avait été consolidée, mais il y avait des ligaments qui avaient été arrachés et qui ne fonctionnaient plus. J'avais donc l'épaule en très mauvais état. J'ai donc été ré-opéré en 2001, et c'est là où tout a été déclenché, on a mis quelqu'un d'autre à mon poste, on m'a viré de mon bureau à un autre, on m'a isolé de mon service. Bien avant j'avais 2 collaborateurs en recherche et développement, et je n'avais plus de notes de services, on les avait passées à mes collaborateurs. Je ne participais plus aux réunions du vendredi, on ne me convoquait plus. Donc ça, ça a été fait sciemment.» (Salarié protégé, délégué syndical, membre du comité d'entreprise, 55 ans, cadre, BEPC, PME, demande de licenciement pour inaptitude)

Se met alors en place un phénomène d'usure : le salarié protégé victime de telles pratiques souhaite alors généralement partir, même s'il dispose d'un soutien au sein de l'entreprise.

«Les délégués m'ont proposé de rester, ils m'ont dit : réfléchis bien, mais si tu veux rester, nous, on fait ce qu'il faut, tu restes. Je ne voyais pas bien comment je pouvais rester dans des conditions pareilles, personnellement, je ne vois pas comment je pouvais travailler dans des conditions

\footnotetext{
"Considérant que le syndic de liquidation des biens de la société Engineering et Ouvrages d'Art n'apporte pas la preuve qui lui incombe qu'ont été recherchés dans les sociétés du groupe Bouygues, pour MM. Authier, Couppe, Duval, Guillemont, Huin et Machado, des emplois équivalents à ceux qu'ils occupaient dans la société Engineering et Ouvrages d'Art, comme cela a été fait pour des salariés non protégés qui avaient des emplois de la même qualification professionnelle que les salariés protégés qui ont été reclassés dans les bureaux d'études de la société Bouygues; que le ministre du travail et de la participation a, par suite, commis une erreur de droit en annulant, au motif susindiqué, la décision de l'inspecteur du travail qui n'est entachée d'aucune illégalité".

${ }^{1}$ Conseil d'Etat, contentieux, 29 décembre 1995, société compagnie européenne pour l'équipement ménager, n 109626 , inédit au recueil Lebon : "Considérant qu'il ressort des pièces du dossier que plusieurs emplois se sont trouvés vacants au sein de l'entreprise ; que si la SOCIETE COMPAGNIE EUROPEENNE POUR L'EQUIPEMENT MENAGER soutient que M. Saadani n'avait pas les qualifications requises pour les occuper, le bien-fondé de cette allégation n'est pas établi, alors surtout que certains de ces emplois ont été proposés à des salariés dont les qualifications n'étaient pas supérieures à celle de M. Saadani ; que, dès lors, la SOCIETE COMPAGNIE EUROPEENNE POUR L'EQUIPEMENT MENAGER n'est pas fondée à soutenir que c'est à tort que, par le jugement attaqué, le tribunal administratif d'Orléans a annulé la décision en date du 16 avril 1987 par laquelle le ministre des affaires sociales et de l'emploi a autorisé la SOCIETE COMPAGNIE EUROPEENNE POUR L'EQUIPEMENT MENAGER à licencier M. Saadani pour motif économique ; ».
} 
pareilles»." (Salarié protégé, membre comité d'entreprise, délégué du personnel, non-syndiqué, 29 ans, cadre, Bac+2, PME, demande de licenciement économique)

Quant aux inspecteurs du travail interrogés, l'un d'entre eux, témoigne également de telles pratiques sans avoir cependant de réelles possibilités de contrôle: l'un fait état de «propositions de reclassements bidons» qu'il a refusées mais les pressions ont été telles sur les salariés qu' «ils ont été licenciés plus tard, sur leur demande; ils n'avaient plus rien à faire ». Leur départ a été négocié. Un autre inspecteur du travail explique avoir eu à se prononcer sur une demande de licenciement pour motif économique qui faisait suite à une nouvelle répartition des tâches dans l'entreprise et constate alors que le poste du salarié "se vidait de sa substance".

De telles évolutions sont également perceptibles au travers de l'analyse de la jurisprudence du Conseil d'Etat : certains arrêts montrent que l'inaptitude du salarié protégé est révélatrice de pratiques que l'on peut qualifier de harcèlement moral et dont la spécificité est de s'inscrire dans la durée. C'est un processus qui est à l'œuvre, ainsi que le «raconte» l'arrêt du Conseil d'Etat.

\section{CONSEIL D'ETAT, 28 JANVIER 1991, MME LEBENTAL Extraits}

Considérant qu'à l'appui de la demande qu'elle a présentée le 30 juin 1986 en vue d'obtenir l'autorisation de licencier Mme LEBENTAL, délégué du personnel et délégué syndical, la société mutualiste MutualitéAccidents-Elèves (M.A.E.) a fait valoir que l'intéressée avait refusé d'accepter une modification substantielle de son contrat de travail comportant un abaissement de sa qualification et de sa rémunération, alors que cette modification était justifiée par la diminution de son aptitude professionnelle ;

Considérant que si Mme LEBENTAL qui avait été embauchée par la société mutualiste MutualitéAccidents-Elèves (M.A.E.) en 1954 et avait atteint en 1973 la qualification de sous-chef de service correspondant à des fonctions de "cadre très qualifié", a été affectée par son employeur à partir de l'année 1983 à des tâches d'exécution de moins en moins importantes, il n'est pas établi que cette diminution progressive de ses responsabilités ait été justifiée par son inaptitude à des fonctions correspondant à sa qualification; qu'en effet, ainsi que l'a retenu le ministre des affaires sociales et de l'emploi dans la décision contestée, il ne ressort pas des pièces du dossier que les capacités professionnelles et la manière de servir de Mme LEBENTAL aient fait l'objet d'observations de l'employeur avant la date à laquelle celui-ci lui a proposé une modification de son contrat de travail ; qu'en admettant même que Mme LEBENTAL n'ait pas elle-même critiqué la diminution progressive de ses responsabilités entre 1983 et 1986, cette circonstance ne saurait être regardée comme équivalent à la reconnaissance tacite par l'intéressée d'une inaptitude professionnelle ; que, par suite c'est à bon droit que, par sa décision du 8 avril 1987, le ministre des affaires sociales et de l'emploi a confirmé la décision de l'inspecteur du travail refusant d'autoriser la société mutualiste Mutualité-Accidents-Elèves (M.A.E.) à licencier Mme LEBENTAL ;

Au bout du compte, si l'état de santé apparaît, parfois, indissociable de la manière dont l'employeur organise la relation de travail, toute la difficulté est alors d'établir un lien juridique entre état de santé, dégradation des conditions de travail, harcèlement moral et, le cas échéant, discrimination. Si la part de subjectivité apparaît importante dans la question de l'appréciation de l'existence d'un harcèlement moral (les mêmes faits peuvent être interprétés de différente manière selon la personne qui les subit, intégrant un certain nombre de paramètres tels que la capacité de résistance, les justifications apportées, les ressources disponibles, notamment personnelles...), la prégnance de la question du harcèlement moral, tant dans sa dimension objective (la prise en compte par le droit) que subjective (le ressenti des salariés protégés) pose aujourd'hui la question de l'adaptation d'un statut juridique construit sur une logique de conflits ouverts. 


\section{II $^{\mathrm{E}}$ PARTIE - L'INTERVENTION DE « TIERS INSTITUTIONNELS » 1}

Les ressources mobilisables par les salariés protégés dans le cadre de leur conflit ne sont pas obligatoirement passives ou internes aux individus. Ces derniers peuvent aussi faire appel au soutien d'autres interlocuteurs, les tiers institutionnels, et principalement, en ce qui nous concerne ici, d'une part l'inspecteur du travail et d'autre part les autres salariés, notamment par le biais du comité d'entreprise.

Le tiers institutionnel désigne les personnes ou les structures interférant dans la procédure bien que n'ayant pas la qualité de partie, au sens juridique du terme. Elles peuvent, en vertu du Code du travail, intervenir directement dans la procédure (l'inspecteur du travail ou encore le $\mathrm{CE}$ ) ou bien accompagner le déroulement de la procédure (les organisations syndicales). Il est alors possible de distinguer la figure de l'inspecteur du travail (I), dont le rôle est central dès lors qu'il rend la décision d'autorisation ou de refus du licenciement, d'interventions que nous qualifierions de "périphériques" et "d'annexes" (II).

\section{I- La figure de l'inspecteur du travail}

Ni l'entreprise, ni le salarié protégé ne peuvent choisir de recourir à tel inspecteur du travail plutôt qu'à tel autre. Le Code du travail a institué des règles précises de compétence rationae loci: l'autorisation de licencier un salarié investi d'un mandat doit être demandée à l'inspecteur du travail dont dépend l'établissement où l'intéressé est employée ${ }^{2}$ Il est de jurisprudence constante que l'établissement pris en compte doit disposer d'une autonomie suffisante. Par conséquent, ce n'est donc pas nécessairement le lieu où le salarié exécute son travail qui est déterminant mais le lieu où est élaboré ou décidé le licenciement ${ }^{3}$.

La figure de l'inspecteur du travail, complexe, est fondamentale dans le déroulement de la procédure entendue au sens large du terme, c'est-à-dire bien en amont de la décision administrative. Garant du respect de la procédure conformément au droit (A), il remplit une fonction de restitution juridique (B) qui n'exclut pas la dimension subjective de la décision (C).

\section{A) Le garant du respect de la procédure}

L'inspecteur du travail apparait, au travers de l'analyse du droit, comme le garant de la mise en œuvre de la protection statutaire. Selon la Cour de cassation, "Le licenciement d'un salarié protégé ne peut être prononcé que pour les faits qui ont motivé l'autorisation

\footnotetext{
${ }^{1}$ L'expression est empruntée à la thèse de R. Mélot, Entre contrat et procès : enquête sur les transactions entre employeur et salarié, Thèse de sociologie soutenue à l'Ecole normale supérieure de Cachan, nov. 2003, 552 p.,

${ }^{2}$ Art. L. 425-I du code du travail s'agissant des DP.

${ }^{3}$ CE 2 février 1006, Société Suburbaine de canalisations et de grands travaux. "Il conviendra qu'une collaboration étroite s'instaure entre les deux inspecteurs [...] l'enquête contradictoire pouvant avoir été effectuée par l'inspecteur du travail du lieu du travail (Circulaire DRT n 3 du $1^{\text {er }}$ mars 2000, Ministère de l'emploi et de la solidarité, Dr. Ouvrier oct. 2000, p. 442 à 451, p. 443).
} 
administrative" $^{1}$; son rôle est donc central. Les entretiens réalisés avec les inspecteurs du travail montrent la place croissante (1) qu'occupe une procédure principalement orale (2).

\section{1) La place croissante occupée par la procédure}

Une place de plus en plus importante semble être accordée, dans les demandes de licenciement de salariés protégés, au droit et au respect de la procédure ; un tel constat renvoie à un phénomène plus global de "procéduralisation" du droit. Des inspecteurs du travail notent ainsi que, de plus en plus fréquemment, le chef d'entreprise s'entoure d'avocats ou de conseils juridiques (même si l'employeur se présente seul aux entretiens contradictoires). En conséquence, l'inspecteur du travail a de plus en plus d'explications à fournir, d'informations à donner. Les discussions débutent généralement sur la question de la compétence ratione loci de l'inspecteur du travail (c'est-à-dire du lieu où est prise la décision, où existe "une autonomie de gestion suffisante" pour décider des mouvements de personnel, généralement le lieu du siège social de l'entreprise). Le Conseil d'Etat lui-même accorde, dans la rédaction de ses considérants, une place importante à la dimension procédurale : "l'autorité administrative doit s'assurer de la régularité de la procédure de licenciement avant de délivrer l'autorisation demandée par l'employeur à l'encontre d'un salarié protégé" ; il s'agit d'une formalité substantielle, prévue à l'article L. 122-32-5 (qui concerne le salarié inapte), "à laquelle est subordonnée la légalité de l'autorisation administrative de licenciement."2.

Un tel constat n'est pas sans incidence sur la manière dont l'inspecteur du travail réalise son contrôle. On peut en effet légitimement penser que les dossiers montés avec le concours de conseils juridiques sont suffisamment bien "ficelés" pour éviter ou contourner les écueils juridiques : la demande de licenciement apparaîtra construite et fondée au plan juridique. Or, le contrôle effectué par l'inspecteur du travail consiste à rechercher si un lien existe entre le licenciement demandé et le mandat du salarié protégé. Prévue à l'article R. 436-7 du Code du travail $^{3}$, cette disposition irrigue l'ensemble des arrêts rendus par le Conseil d'Etat ${ }^{4}$. Elle constitue, par conséquent, la base juridique du contrôle exercé par l'inspecteur du travail en même temps qu'elle structure sa mise en œuvre. On peut penser qu'un dossier argumenté au plan juridique (c'est à dire "qui se tient") donnera lieu à un contrôle que l'on pourrait qualifier d'a minima, l'inspecteur du travail se bornant à vérifier la réalité des motifs invoqués, sans rechercher leur pertinence. Il est vrai que le droit offre, du fait des interprétations possibles des textes, un cadre de contrôle assez souple : selon l'article R. 436-7, l'inspecteur du travail examine "notamment ${ }^{5}$ si la mesure de licenciement envisagée est en rapport avec le mandat". C'est l'emploi de cet adverbe qui permet cette marge d'interprétation. Mais la mise en œuvre du contrôle dépend aussi, par-delà l'interprétation des textes, des personnes et des "stratégies" élaborées.

Certains inspecteurs du travail font le constat de l'existence de dossiers de plus en plus souvent traités en amont, ce qui donne lieu à un "verrouillage". La dimension processuelle du

\footnotetext{
${ }^{1}$ Cass. Soc. 11 juin 2002, n 2011, Claussmann c/ Cie française Eiffel, RJS 8-9/02, n 1001. (s'agissant d'une demande de licenciement pour motif économique devenu licenciement pour faute grave).

${ }^{2}$ CE 22 mai $2002 \mathrm{n}^{\circ}$ 221600, Sté civile d'Arbonne, RJS 8-9/02, $\mathrm{n}^{\circ} 1000$

3 "L'inspecteur du travail et, le cas échéant, le ministre compétent examinent notamment si la mesure de licenciement envisagée est en rapport avec le mandat détenu, brigué ou antérieurement exercé par l'intéressé".

${ }^{4}$ Il est de jurisprudence constante que "Lorsque le licenciement de l'un de ces salariés est envisagé, ce licenciement ne doit pas être en rapport avec les fonctions représentatives normalement exercées ou l'appartenance syndicale de l'intéressé" (lire, par exemple, CE 22 mai 2002, $\mathrm{n}^{\circ}$ 214637, Zaugg, RJS 8-9/02 V, nº 999).

${ }^{5}$ Nous soulignons.
} 
droit du licenciement paraît prendre le pas sur le fond du litige. Cela est particulièrement vrai s'agissant du licenciement pour motif économique où, pour reprendre les termes d'un inspecteur du travail, les petites entreprises "se font ramasser" sur des questions de procédure ; "il est facile de les coincer". À l'inverse, on constate que l'entrée de professionnels du droit, tels les administrateurs judiciaires nommés lors d'une procédure collective (donnant lieu au redressement ou à la liquidation de l'entreprise), dans la procédure contribue à "juridiciser" la procédure. Ces "experts en droit social", pour reprendre les termes de l'un de ceux qui ont été interrogés ne connaissent pas l'entreprise.

"On n'a pas tout l'historique de la société [...] Il y a dû y avoir, antérieurement à notre venue, des difficultés entre le salarié et l'employeur." (Administrateur judiciaire, PME)

Extérieurs à l'entreprise tout en étant provisoirement chargés de sa gestion, leur intervention tend à enfermer le conflit dans un cadre juridique. Il est vrai que seul le droit légitime leur intervention.

Il en résulte un contrôle à "géométrie variable » qui évolue en fonction des motifs du licenciement: le motif économique est plus difficilement contestable, la réalité des faits (suppression de poste) étant souvent établie. Ce constat vaut tout particulièrement pour les demandes de licenciements réalisées lors de procédures collectives : le Plan de sauvegarde de l'emploi (PSE) est généralement a minima étant donné la situation de l'entreprise, "et les inspecteurs du travail le savent", explique un administrateur judiciaire. Le contrôle porte alors moins sur la réalité du licenciement économique que sur les obligations conditionnant la validité du licenciement économique, c'est-à-dire le reclassement et, le cas échant, l'ordre des licenciements, comme le montre la décision du Ministre des affaires sociales, du travail et de la solidarité prise sur recours hiérarchiques (encadré).

\section{DIRECTION DES RELATIONS DU TRAVAIL SOUS-DIRECTION DES DROITS DES SALARIES \\ Bureau des syndicats DS3}

Le Ministre des affaires sociales, du travail et de la solidarité,

Vu les articles L.41218, L.4251> L.4361, L.23611, L.1221416 et

L.5142 du code du travail

Vu le recours hiérarchique formé le 8 juillet 2002 par maître M., mandataire liquidateur de la société R., contre. une décision de l'inspecteur du travail en date du 17 juin 2002, qui refuse d'accorder le licenciement pour motif économique de Madame C.;

$\mathrm{Vu}$ les moyens invoqués à l'appui de la présente requête et après avoir effectué une enquête approfondie ; CONSIDERANT que, le 8 janvier 2002, le Tribunal de Commerce de Paris a ouvert une procédure de redressement judiciaire à l'encontre de la société R. ; que, par un jugement en date du 2 avril 2002, la liquidation judiciaire de la société est prononcée ; que, le 26 avril 2002, le juge commissaire autorise la reprise de la branche d'activité "grand format hors Diazzo" de la société R. par la société NC ainsi que la reprise de la branche d'activité "ruban transfert thermique" par la société R;

CONSIDERANT que la réalité du motif économique est établie; qu'il apparaît que, dans le cadre de cette cession partielle d'activité, le poste de Mme C. qui exerçait des fonctions transversales au sein de R. est supprimé ;

CONSIDERANT que, dans l'hypothèse d'une reprise partielle d'activité, la recherche des possibilités de reclassement doit être effectuée aussi chez les cessionnaires, même si le salarié ne travaille pas dans les secteurs d'activité cédés; qu'en l'espèce, il n'est pas établi qu'un éventuel reclassement de Mme C. était impossible; que, par conséquent, l'administrateur judiciaire, qui n'a pas effectué de démarches particulières pour reclasser cette salariée, n'a pas satisfait à son obligation de recherche de reclassement ; 


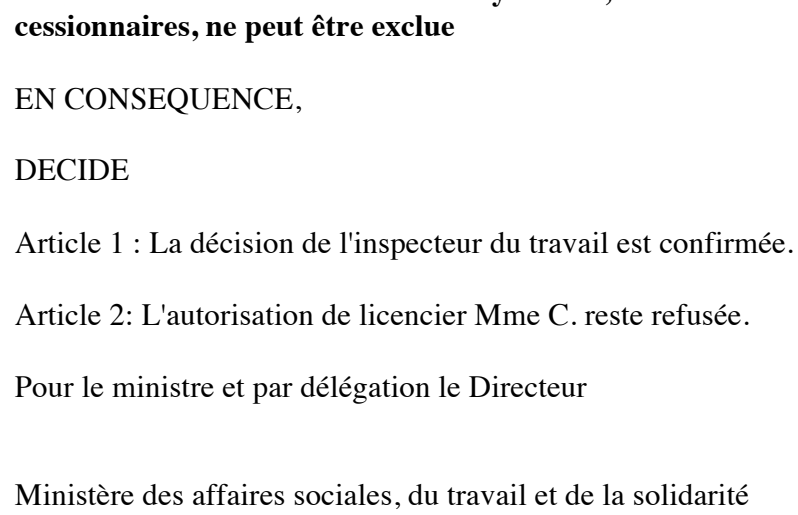

En pratique, nous explique un administrateur, c'est le repreneur qui conditionne la reprise des postes.

"On peut bloquer 6 mois ou 8 mois un licenciement mais au bout du bout, si le dirigeant a décidé de procéder à sa restructuration, il procèdera à sa restructuration." (Administrateur judiciaire, PME)

\section{2) Une procédure principalement orale}

L'enquête contradictoire constitue une phase clé dans le déroulement de la procédure. D'abord imposée par un arrêt Robert du Conseil d'Etat du 3 mars 1954, elle se trouve aujourd'hui inscrite dans le Code du travail depuis un décret du 7 janvier 1959.

\section{Article R. 436-4, $1^{\text {er }}$ alinéa}

"L’inspecteur du travail procède à une enquête contradictoire au cours de laquelle le salarié peut, sur sa demande, se faire assister d'un représentant de son syndicat".

Il est intéressant de relever que lorsque les inspecteurs du travail évoquent l'enquête contradictoire, ils font systématiquement référence à l'entretien individuel qui a lieu à l'Inspection du travail. Un tel "raccourci" terminologique rend parfaitement compte de l'importance de cette phase dans la prise de décision de l'inspecteur du travail. Bien que qualifiée, par le droit, de "contradictoire", c'est-à-dire imposant aux parties la communication de toutes les pièces qui devront ensuite être débattues, l'enquête demeure confidentielle et ne donne pas lieu à la confrontation des parties ${ }^{1}$. On peut alors parler d'un principe contradictoire atténué : dans ce contexte de "confidentialité", l'inspecteur du travail devient le principal garant du respect du principe contradictoire. Ce constat, issu de l'analyse des pratiques, est assez révélateur des représentations attachées au déroulement de la procédure et de la place occupée par l'inspecteur du travail.

L'inspecteur du travail se doit d'entendre les deux parties, l'entretien individuel avec le seul salarié ne pouvant, sous peine de nullité de la décision, tenir lieu d'enquête contradictoire².

\footnotetext{
${ }^{1}$ L'inspecteur du travail n'est pas tenu d'organiser une confrontation entre les parties (CE 20 fév. 1995, Layma, req. $\mathrm{N}^{\circ}$ 123.757), ni d'informer chacune des parties des éléments apportés par l'autre (CE 8 janvier 1982, Et. Petitjean, Req. $\mathrm{N}^{\circ}$ 21.814).

${ }^{2}$ CE 29 déc. 1997, Société anonyme Le Comptoir Général Maritime, nº 160604.
} 
L'entretien doit couvrir l'ensemble des motifs avancés par l'employeur à l'appui de sa demande de licenciement, l'inspecteur du travail devant, au besoin, convoquer à nouveau les parties $^{1}$. L'audition doit être personnelle et individuelle ${ }^{2}$ et ne peut être remplacée par un entretien téléphonique ${ }^{3}$. Suivant la circulaire DRT $\mathrm{n}^{\circ} 3 \mathrm{du} 1^{\text {er }}$ mars 2000, Ministère de l'emploi et de la solidarité, il convient d'"insister tout particulièrement sur l'audition des parties et sur la rédaction d'un compte-rendu d'audition qui, sans être une transcription littérale, devra néanmoins s'efforcer de rapporter d'une manière exhaustive les informations recueillies et les positions de chacune des parties entendues" ${ }^{4}$. Une telle position se justifie d'autant plus que le recours hiérarchique, c'est-à-dire formé auprès du Ministre chargé du travail, est reçu par les services de la Direction des relations du travail (DRT) ensuite chargé "d'effectuer une analyse juridique de l'ensemble du dossier transmis, d'en établir une synthèse dans un "avis du service" présentant le dossier, et enfin de proposer une décision" au ministre. L'analyse de la jurisprudence du Conseil d'Etat montre l'importance accordée, au plan juridique, à l'enquête contradictoire. Elle doit permettre au salarié protégé de " [...] faire valoir $[\ldots]$ ses arguments pour contester un licenciement..." ${ }^{6}$ 'inspecteur du travail dispose, selon la loi ${ }^{7}$, d'un délai de 15 jours pour statuer ${ }^{8}$.

C'est ainsi que les décisions rendues peuvent tirer les conséquences juridiques qui s'imposent d'une absence à l'entretien préalable.

\author{
INSPECTION DU TRAVAIL \\ 20ème Section \\ Nanterre, le 29 novembre 2002 \\ REF \\ DECISION \\ L'Inspecteur du Travail, par intérim, soussigné XX.,
}

\footnotetext{
1 "Considérant qu'à l'appui de sa demande initiale à l'inspecteur du travail la société Serco a invoqué les fautes commises par Mlle Gonzalez; qu'il résulte des pièces du dossier que si la société a ensuite ajouté à sa demande initiale un motif tiré de l'inaptitude physique de Mlle Gonzalez, cette dernière n'a pas été mise à même de faire valoir, lors de l'enquête contradictoire diligentée par l'inspecteur du travail, ses arguments pour contester un licenciement fondé sur ce motif ; que dès lors l'inspecteur du travail ne pouvait fonder sa décision sur le seul motif de l' inaptitude physique de Mlle Gonzalez sans entacher sa décision d'irrégularité" (CE n 118731, 7 fév. 1992, société Serco, non publié).

${ }^{2} \mathrm{CE} 28$ avril 1997, $\mathrm{n}^{\circ} 163971$.

${ }^{3}$ CE 21 août 1996, n 149249.

${ }^{4}$ Dr. Ouvrier oct. 2000 , p. 442 à 451, p. 443.

${ }^{5}$ Circulaire DRT $n^{\circ}$ 2003/16 relative au traitement des recours hiérarchiques formés contre les décisions des inspecteurs et des directeurs du travail en matière de salariés protégés et d'élections professionnelles, 3 octobre 2003, p. 3. La circulaire évoque cependant l'éventualité d'une demande d'audition par l'une des parties lors du recours hiérarchique. "Dans ce cas, il est d'usage de la recevoir et de contacter l'autre partie afin, dans le souci du respect d'une procédure contradictoire, de lui proposer, si elle le souhaite, de la recevoir également". Par ailleurs, les services déconcentrés (directeur régional ou directeur départemental) procèdent, dans le cadre du recours hiérarchique, à une contre-enquête qui se traduit, notamment, par l'audition des parties.

${ }^{6}$ Conseil d' Etat, 7 février 1992, Société Serco.

${ }^{7}$ Art. R. 436-4, 2e al. du Code du travail.

${ }^{8}$ En pratique, il est intéressant de noter que ce délai est quasiment toujours prolongé, justifié, dans la lettre que l'inspecteur du travail envoie aux parties, par "les nécessités de l'enquête", c'est-à-dire conformément à la formule lapidaire du Code du travail, sa contextualisation. En pratique, on constate que c'est une précaution que prennent systématiquement les IT : "quand on démarre l'enquête, on ne sait jamais si on va la terminer dans le délai. Donc, par précaution, pour ne pas se faire piéger par un pur problème de forme, parce que le jour où on doit prolonger, soit on oublie, soit on est à l'extérieur, soit on n'a pas de secrétaire, etc... Quand on ne sait pas, on prolonge systématiquement, ce qui ne veut pas dire qu'on l'utilise systématiquement" (Inspecteur du travail).

En tout état de cause, une telle prolongation est, selon le Conseil d'Etat, sans incidence sur la légalité de l'autorisation de licenciement.
} 
Vu la demande d'autorisation de licenciement de Monsieur Y. Conseiller du salarié et ancien délégué
syndical, présentée le 29 octobre 2002 par la société X sise [...], au motif suivant: abandon de poste non
justifié depuis le 14 octobre 2002 ,
Vu les articles L $122-14-16$, L $412-18$ et R $436-1$ et suivants du Code du Travail,
Vu la convocation à l'entretien préalable du 22 octobre 2002 ,
Vu la prolongation du délai d'enquête notifiée aux parties le 5 novembre 2002,
Vu les éléments recueillis lors de l'enquête effectuée le 15 novembre 2002,
Considérant que Monsieur Y., convoqué à fins d'enquête contradictoire par courriers des $\mathbf{5}$ et 19
novembre 2002, n'a pas souhaité répondre à cette invitation,
Considérant en conséquence, à défaut d'arguments présentés par le salarié, que résulte des éléments
recueillis lors de l'enquête la réalité du motif invoqué,
Considérant que ce motif constitue une faute de nature à justifier le licenciement de Monsieur T.,
Considérant que la demande d'autorisation de licenciement est sans lien avec les mandats détenus par
l'intéressé,
DECIDE

L'issue de l'enquête contradictoire est conditionnée par l'apport des parties, c'est-à-dire leur contribution et leur investissement personnels. Le contrôle de l'inspecteur du travail s'appuie sur les "contributions", inégales, des parties. Elles varient, dans les formes et sur le fond, suivant que l'on a affaire à l'employeur ou au salarié. Placé dans la situation juridique du demandeur, l'employeur est tenu de motiver sa demande et donc d'exposer les motifs qui justifieraient, selon lui, le licenciement du salarié protégé. Sa demande apparaît, par les analyses de dossiers déjà réalisées, étayée ; elle s'appuie sur un certain nombre de documents écrits fournis à l'inspecteur du travail et dont la nature et le nombre varient suivant le motif du licenciement. L'employeur peut ainsi se préconstituer un certain nombre de "preuves" à l'appui de sa demande (dont il maîtrise le déclenchement). Il arrive que des dossiers de demandes de licenciement apparaissent "construits", revêtus en quelque sorte d'un "habillage juridique". Le salarié se trouve, quant à lui, dans la position de défendeur. Exception faite des cas de discrimination syndicale ou de harcèlement professionnel, le salarié ne semble pas préconstituer de dossier. Ce faisant, l'entretien personnel réalisé avec l'inspecteur du travail se révèle alors être le lieu privilégié où le salarié va livrer sa version du litige. Face à une personne extérieure à l'entreprise, dans un lieu "neutre" et hors présence de l'employeur ou d'un de ses représentants, la parole du salarié se trouve libérée. L'analyse de certains dossiers montre que l'inspecteur du travail peut "fonder son intime conviction" à partir d'éléments qui, afin de préserver des intérêts tiers (salariés demeurés dans l'entreprise, par exemple) et conformément au droit, demeureront confidentiels et n'apparaîtront pas dans la motivation ${ }^{1}$. L'analyse des pratiques montre qu'une large partie de l'issue du conflit peut se jouer lors de l'entretien préalable dès lors qu'il permet que se libère la parole.

\footnotetext{
${ }^{1}$ Selon le Conseil d'Etat, l'inspecteur du travail, tenu d'un devoir de réserve, n'est pas "tenu de communiquer à la société l'ensemble des témoignages qu'il avait recueillis au cours de l'enquête contradictoire" (Conseil d'Etat 3 fév. 1992, Sté Bonny, $\mathrm{n}^{\circ} 113890$, non publié).
} 
"Il est évident que même si vous connaissez une partie des réalités de l'entreprise, il y a une bien plus grande partie qui vous échappe, mais cette partie-là, on compte aussi sur le salarié pour vous l'apporter lors de l'enquête contradictoire. C'est-à-dire que si lui pense qu'il y a un lien avec le mandat, vous lui dîtes en quoi mais vous lui demandez aussi les éléments objectifs, donc on veut des courriers, etc... Donc vous lui donnez un rendez-vous suivant, ou il vous les envoie (...) on compte sur lui pour se défendre, c'est son emploi qui est en jeu. Et s'il ne veut pas se défendre, on ne va pas le sauver malgré lui, sauf si bien sûr on a déjà tous les éléments dans le dossier." (Inspecteur du travail)

Un autre inspecteur du travail résume cette phase clé.

"Je me fais une opinion en fonction des éléments d'information qu'on me communique d'un côté et de l'autre. [...] Après, moi j'ai compilé une masse de documents qu'on m'a fourni d'un côté et de l'autre, et j'ai trouvé des éléments qui n'étaient pas très cohérents dans la position de la direction qui avait pourtant un bon dossier globalement. Mais j'ai utilisé les éléments que je n’estimais pas très cohérents pour tirer du côté du refus." (Inspecteur du travail)

Les inspecteurs du travail interrogés font état d'auditions (employeurs et salariés) réalisées séparément et généralement au sein des locaux de l'inspection du travail, un lieu neutre. Un inspecteur du travail justifie ses choix.

"J'entends toujours le salarié plutôt après l'employeur, c'est quand même lui qui va être viré, parce
qu'on a beau dire, on est tous influencés. Je m'aperçois à quel point il y a une différence, quand on
entend l'employeur et on entend le salarié qui donne une autre version. Et après, moi, ma
démarche, c'est d'aller lorsque la divergence est sérieuse, lorsqu'on a véritablement deux versions
qui s'opposent d'aller en enquête derrière, sur de la recherche de documents ou par interview
d'autres personnes." (Inspecteur du travail)

Certains inspecteurs du travail procèdent à une confrontation lors de demandes de licenciement pour faute grave, afin de mettre les parties à même de s'expliquer. C'est au cours de l'entretien préalable que l'inspecteur du travail peut "contextualiser" le litige et inscrire le conflit dans son histoire.

\section{Décision administrative de licenciement rendue par un inspecteur du travail Extrait}

Vu la demande d'autorisation de licenciement pour motif $\mathbf{d}^{\prime}$ 'inaptitude ${ }^{1}$ au poste de travail, présentée le 11 juillet 2002 par l'entreprise D. et concernant Monsieur Y., élu du comité d'entreprise, délégué du personnel, membre du CHSCT et délégué syndical CGC ;

Vu les articles L 425-1, L 436 1, R 436-1 et suivants du code du travail ;

Vu la procédure d'entretien préalable qui a eu lieu le 2 juillet 2002 ;

$\mathrm{Vu}$ l'avis du comité d'entreprise en date du 8 juillet 2002 ;

Vu la prolongation du délai d'enquête notifiée le 15 juillet 2002 ;

Vu les éléments recueillis lors de l'enquête contradictoire du 19 juillet 2002 ;

Considérant la réalité du motif d'inaptitude médicale;

Considérant néanmoins que l'enquête a permis de constater que la survenance de cette inaptitude s'explique essentiellement par un litige ancien et non réglé sur le contrat de travail et le poste de Monsieur Y.;

C'est également au cours de l'enquête contradictoire que l'inspecteur du travail vérifie les faits à l'appui de la demande de licenciement et peut procéder, si cela est pertinent, à certaines investigations comme, par exemple, entendre les autres salariés. Des révélations sont parfois

\footnotetext{
${ }^{1}$ Nous soulignons.
} 
faites, mais qui ne peuvent servir de base à l'appui de la décision (exemple de salariés à qui l'on a "forcé" la main pour qu'ils signent une pétition contre le salarié protégé dont l'employeur demande le licenciement).

"Il faut éplucher et à chaque fois qu'on trouve des points sur lesquels il y a des possibilités d'interprétation ou des marges d'incertitude, il faut essayer de les lever. (Et donc vous demandez des informations complémentaires à chaque fois ?) À chaque fois, à la société avec souvent une contre-expertise de la part du salarié. (Une contre-expertise, c'est-à-dire ?) Je lui demande : "votre direction me dit ça à partir de tel document, qu'est ce que vous avez à dire là-dessus ?" (Inspecteur du travail)

On voit donc bien que l'enquête contradictoire permet de rétablir un équilibre dans le litige opposant l'employeur et le salarié ; elle constitue une phase clé dans la prise de décision de l'inspecteur du travail.

En tout état de cause, le souhait du salarié protégé de quitter l'entreprise pèse très lourd dans la prise de décision de l'ensemble des inspecteurs du travail interrogés.

\begin{abstract}
"Pour une décision de licenciement, on prend plusieurs choses en compte, on prend la réalité du motif, la procédure, les efforts de reclassement, les liens avec le mandat et l'intérêt général. Mais quand vous avez un salarié qui vous dit : moi je n'en peux plus, je ne suis pas prêt à me battre, je ne suis pas prêt à m'imposer, je ne souhaite qu'une chose c'est changer d'air, vous n'allez pas l'imposer en sachant que vous mettez un autre élément de sa santé en jeu : sa santé mentale. Parce que si le gars est harcelé, s'il est stressé et fait une déprime à la sortie, quel est l'intérêt ? [...] À partir de là, on ne peut pas se montrer plus royaliste que le roi, même si, la demande du salarié doit être prise en compte vraiment en dernier ressort : un salarié qui vous dit, je veux être licencié, ce n'est pas pour autant qu'on va accorder l'autorisation de licenciement, mais s'il vous dit, je veux être licencié parce que je vais craquer mentalement parce que c'est du harcèlement permanent, je crois que là c'est logique d'accorder une autorisation de licenciement." (Inspecteur du travail)
\end{abstract}

\title{
B) Une fonction de restitution juridique
}

En donnant aux faits leur qualification juridique (1), les inspecteurs du travail remplissent un rôle de "restitution juridique". Ils représentent une autorité qui dispose d'un pouvoir encadré au plan juridique (2).

\section{1) La qualification juridique donnée aux faits}

Le contrôle de l'inspecteur du travail s'inscrit dans un processus qui permet de traduire des faits en droit. Il joue le rôle de "passerelle" entre les faits et le droit, objectivant le conflit dans le litige. L'exemple peut aussi être donné s'agissant du médecin du travail qui "transforme" l'incapacité, observation clinique, en inaptitude, catégorie juridique. Il réalise alors, en normalisant le conflit, une médiation entre les faits et le droit.

L'appréhension des faits occupe, dans cette procédure, une place fondamentale. Pour ce faire, l'inspecteur du travail s'appuie, ainsi que nous venons de le voir, sur l'entretien préalable, mais aussi une connaissance intime de l'entreprise et du contexte économique de la région qu'il possède dès lors qu'il est en poste depuis un certain temps. Le rôle des "précédents" peut alors, lorsqu'ils existent, intervenir dans la prise de décision : importance des plaintes pour 
violation du Code du travail déposées par les salariés, par exemple, ou encore, ainsi qu'en témoigne un inspecteur du travail, le fait que :

"D’autres cadres avaient démissionné, étaient partis." (Inspecteur du travail)

Le contrôle de l'inspecteur du travail suit plusieurs étapes :

- vérification de la réalité des faits : les faits sont-ils, dans un licenciement pour motif personnel, imputables au salarié ? Le poste a-t-il été supprimé (motif économique) ?

- recherche d'un éventuel lien avec le mandat : les motifs avancés par l'employeur ont-ils été déterminants dans la volonté de licencier ? ; la cause qui motive la demande est-elle sérieuse?

- prononcé de la décision qui doit être motivée.

L'analyse des décisions d'acceptation rendues par les inspecteurs du travail permet de les classer dans plusieurs catégories en fonction du choix réalisé quant au type de rédaction retenu. La rédaction de la décision dépend en effet de la nature de la décision (acceptation ou rejet) et du contexte dans lequel a été prise la décision (volonté ou non de partir du salarié protégé). Un essai de typologie est alors possible. On peut distinguer :

1) Les décision stéréotypées qui ont été prises dans le cadre d'un consensus sur la qualification juridique de la rupture du contrat de travail.

2) Les décisions avec réserve : elles accordent le licenciement parce que le salarié souhaite partir, mais elles laissent apparaître les motifs d'irrégularité qui auraient pu conduire l'inspecteur du travail au refus (lire l'exemple de décision dans l'encadré cidessous).

3) Les décisions "constructives" : elles statuent sur la demande, mais, dans le même temps, relèvent certaines irrégularités commises par l'entreprise.

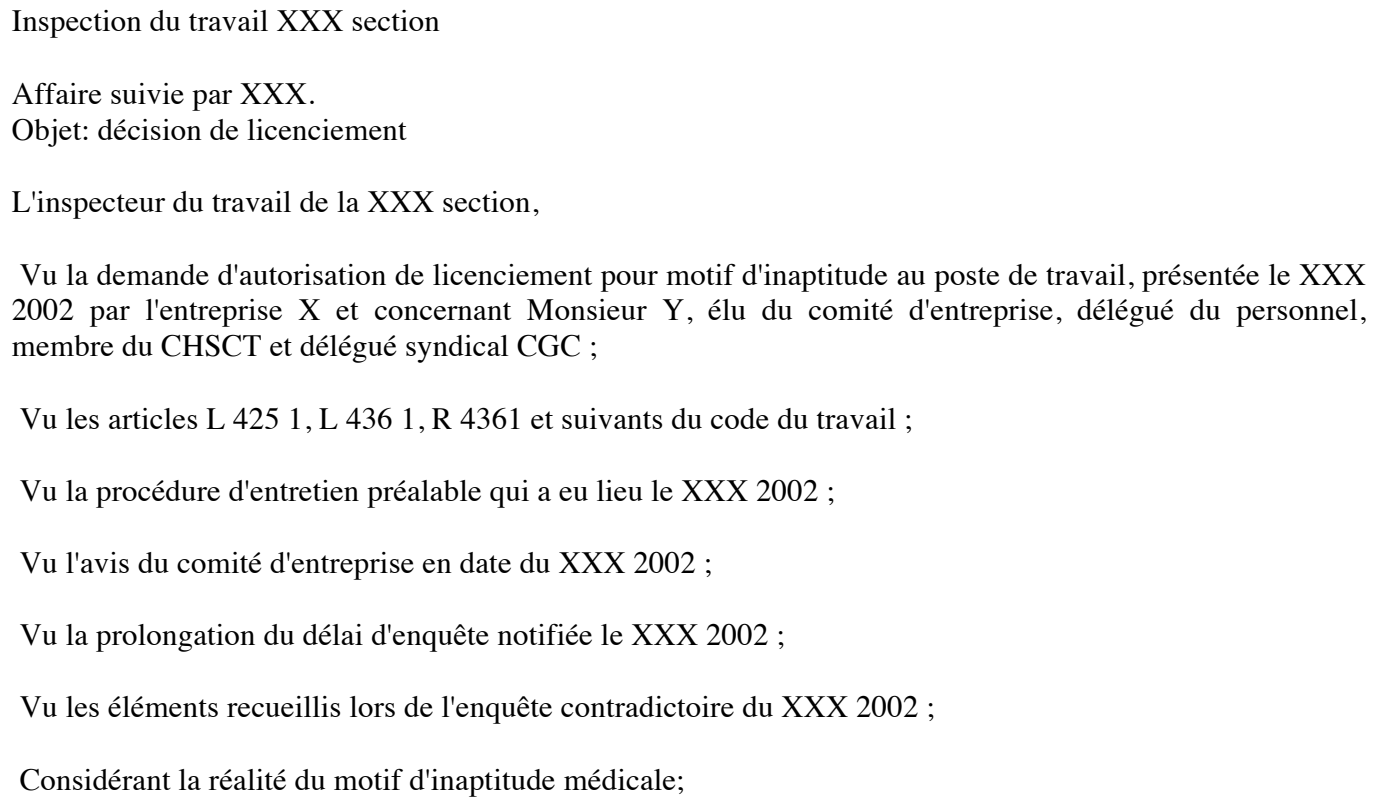




\begin{abstract}
Considérant néanmoins que l'enquête a permis de constater que la survenance de cette inaptitude s'explique essentiellement par un litige ancien et non réglé sur le contrat de travail et le poste de Monsieur Y;

Considérant que l'employeur a alors modifié unilatéralement le contrat de Monsieur Y restreignant ses responsabilités dans une ambiance à la tension croissante, proche de la définition légale du harcèlement moral au travail, alors même que du fait de ses mandats aucune modification unilatérale ne pouvait être imposée à Monsieur Y.
\end{abstract}

Considérant que ces faits permettraient de motiver une décision de refus d'autorisation de licenciement $^{1}$;

Considérant cependant qu'au cours de l'enquête Monsieur Y a confirmé qu'il estimait désormais impossible le retour à une situation de travail normale et qu'il souhaitait un licenciement pour inaptitude assorti dune transaction mettant fin aux procédures civiles engagées

Considérant l'impossibilité d' «assurer le reclassement de Monsieur Y dans l'entreprise,

DECIDE

L'autorisation de licenciement de Monsieur Y est accordée.

Fait à XXX, le XXX 2002

L'Inspecteur du travail

de la XXX section

La motivation donnée à la décision constitue la traduction du passage des faits au droit. Elle est, au plan juridique, fondamentale dès lors qu'étant obligatoire elle permet que s'exerce le contrôle sur la décision et constitue un gage d'impartialité ${ }^{2}$. À cet égard, il est intéressant de noter que les pratiques des inspecteurs du travail divergent selon les objectifs qu'ils se fixent.

"La motivation doit être la plus simple possible, et qu'elle vous conduise systématiquement à cette décision" ("Pourquoi la plus simple possible, car on pourrait penser qu'il faut au contraire expliquer beaucoup pour que les parties la comprennent ?) C'est ce que je faisais avant. Je me rappelle quand j'étais en formation, j'avais fait une décision de 5 pages, je pensais que ma décision serait inattaquable, et je me le dis encore aujourd'hui, mais il y avait au moins sur une page quelque chose qui ne devait pas tenir la route. Plus vous en mettez, plus vous avez des chances, sur un point au moins de n'être pas au top et donc de vous faire casser. Donc, je dis que ce n'est pas la peine d'accumuler, il faut aller au plus simple possible." (Inspecteur du travail)

\title{
La pratique est différente pour un autre inspecteur du travail.
}

"J'aime assez bien détailler, je parle des dossiers qu'on étudie vraiment, pas ceux où on sait qu'il n'y aura pas débat. Ceux vraiment qu'on a travaillé, je choisis en général d'être assez descriptif dans la motivation. D'abord parce que ça construit d'avance le travail qu'on doit faire du rapport s'il y a un recours hiérarchique. Ça peut aussi dissuader de le faire, pas toujours malheureusement, si on pense qu'on a développé des choses assez pertinentes, moi je me suis dit, après tout celui qui

\footnotetext{
${ }^{1}$ Nous soulignons.

${ }^{2}$ Il est intéressant de noter que dans les années 50 "En pratique, les décisions des inspecteurs revêtent une forme quasi juridictionnelle et sont généralement fortement motivées : il n'est pas rare de trouver dans les archives du ministère du Travail des décisions portant sur des licenciements collectifs faisant une dizaine de pages. Ces pratiques sont largement déterminées par les circulaires officielles et les recommandations plus officieuses du ministère qui attache une grande importance à montrer un souci d'impartialité et de rigueur dans l'examen des demandes." (J.-P. Le Crom, L'introuvable démocratie salariale. Le droit de la représentation du personnel dans l'entreprise (1890-2002), Paris, Syllepse, coll. Le Présent Avenir, 2003, 194 p., p. 97).
} 
le reçoit, l'employeur, c'est son droit d'ailleurs d'avoir un acte motivé, je trouve que c'est normal, ça peut le dissuader si ce que j'ai développé est construit." (Inspecteur du travail)

\title{
2) Une autorité qui dispose de pouvoirs encadrés
}

Le droit a encadré les pouvoirs dont dispose l'inspecteur du travail. Il existe un droit de recours sur la décision prise (recours administratif, gracieux ou hiérarchique, et/ou recours contentieux). Les personnes à qui la décision fait grief peuvent demander l'annulation de la décision de l'inspecteur du travail devant les tribunaux administratifs. D'autre part, la jurisprudence a défini des limites aux pouvoirs de l'inspecteur du travail. Si elle lui reconnaît la faculté de retenir, pour refuser d'accorder un licenciement, "des motifs d'intérêt général relevant de son pouvoir d'appréciation de l'opportunité", c'est "sous réserve qu'une atteinte excessive ne soit pas portée à l'un ou à l'autre des intérêts en présence" ${ }^{11}$. L'inspecteur du travail peut, d'autre part, voir sa responsabilité engagée dès lors qu'il a agi pour des motifs étrangers à l'intérêt général ${ }^{2}$. D'autre part, s'il incombe à l'inspecteur du travail de procéder à une analyse circonstanciée de la situation et de vérifier la matérialité des faits, il n'en demeure pas moins lié par les termes de la demande d'autorisation de licenciement. Autrement dit, il ne peut retenir de griefs qui n'auraient pas été invoqués par l'employeur ni s'abstenir de statuer sur certains griefs ${ }^{3}$.

Investi de pouvoirs encadrés mais considérables, l'inspecteur du travail apparaît comme une "autorité". Il est, suivant l'étymologie du terme, à la fois le fondateur, l'instigateur, le conseiller et aussi l'auteur... C'est-à-dire qu'il dispose du "pouvoir d'imposer l'obéissance" ${ }^{4}$. Le Procureur général Touffait se référait, sous les fameux arrêts Perrier, au fait qu'ils " [...] possèdent en outre, en général, une grande autorité morale dans les entreprises qu'ils surveillent"

C'est ce que l'on retrouve indirectement exprimé par M.P., salarié protégé.

\begin{abstract}
"Pour nous [les salariés], inspecteur, c'est un nom qui est assez imposant, c'est vrai qu'on pense tout de suite : respect. Et il fait appliquer les lois, il fait envoyer les PV." (Salarié protégé, membre du comité d'entreprise et du CHSCT, syndiqué, agent de maîtrise, 28 ans, BTS, PME, demande de licenciement pour inaptitude)
\end{abstract}

C'est également l'image que renvoie un autre salarié protégé, raconté en des termes très forts.

\begin{abstract}
"Je pense que [le Directeur Général] avait quand même des craintes de ce côté-là, sans savoir ce qui allait lui arriver. Je sais qu'une fois, Monsieur M. [l'inspecteur du travail] était monté, il a dit à la standardiste (c'est elle qui me l'a rapportée) : je voudrais voir Monsieur G., Donc elle téléphone : Monsieur G. ? Monsieur M., l'inspecteur du travail est là. Il répond : dites-lui que j'ai des rendezvous toute la journée. Alors Monsieur M. lui a fait dire qu'un inspecteur du travail vient quand il veut et sans rendez-vous, alors dites lui qu'il annule ses rendez-vous et qu'il est à ma disposition. Ça ne lui avait pas plu, mais c'est comme ça que ça c'était fait. Je pense que Monsieur M., cette entité "inspection du travail" à travers un homme qui sait ce qu'il veut et qui connaît bien son boulot, ça leur a fait lever un peu le pied sur la pédale quand ils ont su que tout s'engageait par là.
\end{abstract}

\footnotetext{
${ }^{1}$ CE 1 février 1989 - N 64575, Boistard. Ces motifs d'intérêt général qui peuvent consister à maintenir une représentation du personnel dans l'entreprise (CE 22 juin 1987, $\mathrm{n}^{\circ}$ 74627), ou encore prévenir une augmentation des tensions sociales dans et hors de l'entreprise (CE 21 décembre 1994, n 149089).

${ }^{2}$ CE 6 janvier 1989 - N 84757, Société Automobiles Citroën

${ }^{3}$ CE 16 juin 1996, Sevestre.

${ }^{4}$ Le terme est emprunté au latin auctoritas, dérivé de auctor (A. Rey, Dictionnaire précité).

${ }^{5}$ Cass. Chambre mixte 21 juin 1974, Perrier, Dalloz 1974, p. 593, Concl. Touffait, p. 597.
} 
(Et vous pensez que c'est le seul fait que ce soit cet inspecteur du travail ou que ç'aurait pu être pareil avec un autre inspecteur ?) Ce n'est pas Monsieur M. mais l'inspection du travail." (Salarié protégé, délégué syndical, membre du comité d'entreprise, 55 ans, cadre, BEPC, PME, demande de licenciement pour inaptitude)

Le point de vue porté sur l'inspecteur du travail dépend, dans une certaine mesure, du rapport que le salarié protégé entretient avec l'autorité ; l'inspecteur du travail est aussi celui avec qui il faut composer.

"Donc on avait demandé à [l'inspecteur du travail], parce que lui peut l'imposer [la mise en
place d'un CHSCT], il a le droit, ça fait partie de ses prérogatives, il peut très bien l'imposer.
Donc on a été le voir dans cet esprit-là, et on n'a sans doute pas été très psychologue sur le coup,
on ne s'est pas rendu compte qu'on avait affaire à quelqu'un qui était en vue de ses prérogatives,
qui était "un monsieur qui sait". Il faut laisser le "'monsieur qui sait"'parler, il ne faut pas lui
montrer qu'on sait aussi et qu'on peut le mettre en difficulté. Et là, on a eu un petit peu tort parce
qu'on a été un petit peu trop loin, on lui a montré que lui avait le droit. Et le problème, c'est que,
d'après ce qu'il nous a expliqué, il s'est déjà fait planter. Comme ça une fois devant les tribunaux
où il essayait d'imposer un CHSCT et il s'est fait débouter par les tribunaux. Donc il n'a pas voulu
y retourner." (Salarié protégé, membre du comité d'entreprise, délégué du personnel, syndiqué, 37
ans, cadre, Bac+2, PME, demande de licenciement pour faute)

L'inspecteur du travail est parfois une ressource pour le salarié. Il lui sert alors de cadre de références, voire d'argument d'autorité, ainsi que l'explique un Directeur général.

"Et puis j'ai l'impression que P. voyait [l'inspecteur du travail] très souvent, puisque chaque fois que P. venait, il disait : j'étais à l'inspection du travail, il faut que vous fassiez ça, alors moi : "on se calme, ce n'est pas vous qui allez me dire ce qu'il faut faire, j'ai un patron, et s'il faut respecter la loi, $\mathrm{j}$ 'ai des conseils et ce n'est pas vous qui me donnerez la marche à suivre..." (Directeur général, PME)

Il peut s'engager une véritable coopération entre l'inspecteur du travail et le salarié protégé, comme l'explique ce dernier.

"J'ai donc informé [l'inspecteur du travail] que la situation tendait à se dénouer un petit peu, que le dialogue s'était établi, qu'il pouvait mettre en instance ce que je lui avais demandé, que ça ne risquait plutôt d'interférer dans la façon de faire de l'employeur et de le braquer. Et que, par contre, je reprendrais contact avec lui si les choses ne se passaient pas bien, donc j'ai gardé la porte ouverte, mais là, ce n'était plus la peine qu'il se dérange puisque les choses avaient l'air d'aller mieux." (Salariée protégée, déléguée du personnel, non-syndiquée, 55 ans, profession intermédiaire, sans diplôme, TPE, demande de licenciement économique)

\section{L'inspecteur du travail est, plus rarement, une ressource pour l'entreprise.}

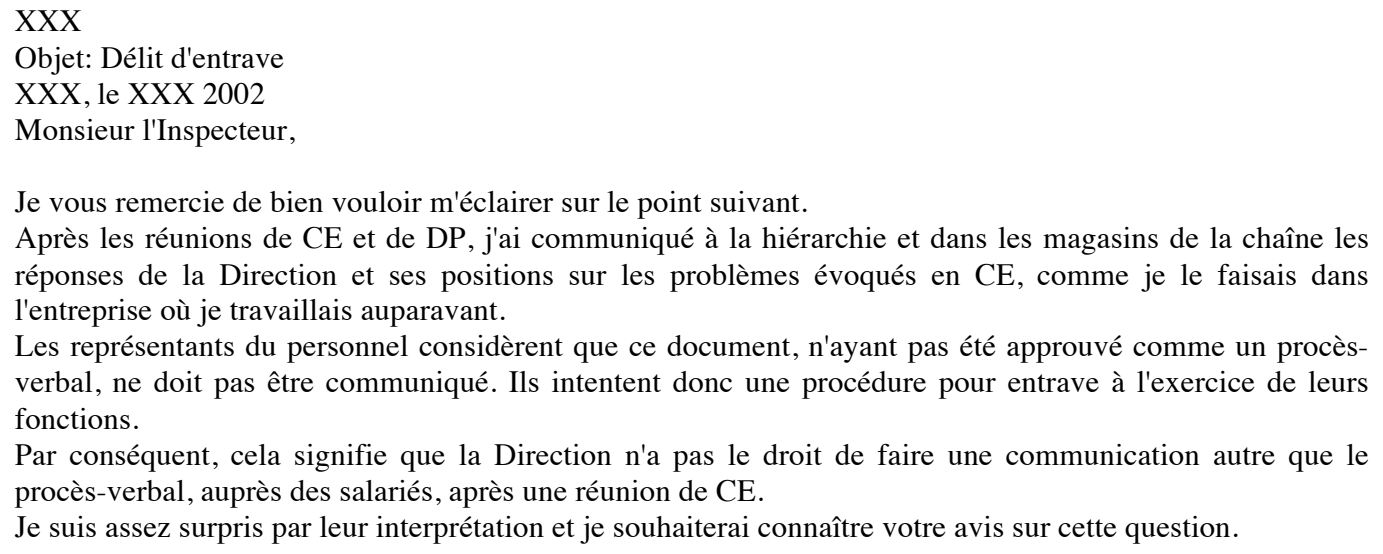


En vous remerciant de l'attention que vous porterez à ce courrier,

Je vous prie d'agréer, Monsieur l'Inspecteur, l'expression de mes salutations distinguées

Direction des ressources humaines

Un inspecteur du travail résume assez bien le rôle effectivement joué par l'inspecteur du travail.

"Je pense qu'en réalité, l'échange est en fonction de la demande." (Inspecteur du travail)

L'autorité de l'inspecteur du travail est souvent mal acceptée par les dirigeants d'entreprise qui la perçoivent comme une atteinte à leurs pouvoirs. Un Directeur général fait état de l'"acharnement" de l'inspecteur du travail, d'autant moins compréhensible dès lors que le salarié veut partir.

Les inspecteurs du travail jouissent d'une réputation auprès des employeurs et des salariés : interrogé sur l'intervention de l'inspecteur du travail, un directeur de société nous répond :

"Je peux vous faire une réponse claire, ça dépend de l'inspecteur du travail. J'en ai connu
plusieurs, j'ai travaillé dans plusieurs régions, j'en ai connu qui s'en foutent complètement, ils
vous signent n'importe quoi. Celui de X. est droit comme la justice, donc ça dépend du bonhomme
qu'on a en face, celui de Y. est raide, je peux vous le dire." (Directeur établissement, PME)

Cette réputation conditionne la manière dont va être construit le dossier de demande de licenciement.

"Je suis persuadé que [le pourcentage de recours ou de demandes] est intimement lié à des
phénomènes de réputation locale. C'est-à-dire que moi j'ai un "taux-échec" en recours qui est très
faible globalement, probablement parce que en amont de tout ça, ce n'est pas que je sois forcément
meilleur que les autres, c'est surtout que je ne me suis pas cassé les dents pendant un certain temps
et donc ça ce sait sur la place, et donc les employeurs ne viennent pas me voir avec n'importe quel
dossier. S'ils pensent que le dossier est à moitié foireux, ils ne présentent pas le dossier."
(Inspecteur du travail)

\section{C) La dimension subjective de la décision de l'inspecteur du travail}

Il convient de noter que le travail des inspecteurs du travail peut présenter d'importantes différences. En effet, ces derniers peuvent effectuer leur activité dans un autre ministère, ce qui place l'inspecteur du travail dans une configuration singulière, ainsi que le montre l'exemple donné ci-dessus :

"(Je suis) le seul inspecteur du travail pour toute la branche des transports sur le département (avec) des contacts plus ou moins épisodiques (avec mes collègues). Aux transports, on n'a pas défini une règle stricte de seuil d'effectif de salariés, mais on a réparti entre nous les entreprises de transport, de façon à ce que, notamment les contrôleurs qui ont une certaine ancienneté puissent aussi avoir des entreprises plus importantes." (Inspecteur du travail)

Les changements de fonction, la mobilité spatiale des inspecteurs du travail, les remplacements pendant les absences tout comme la manière dont chacun délègue plus ou moins de tâches aux contrôleurs, comme les cas ci-dessus, impliquent que la connaissance du contexte professionnel peut être assez différente d'une affaire à une autre. 


\begin{abstract}
"Je venais d'arriver sur la section, donc je n'ai pas une grande connaissance des entreprises, enfin, surtout à l'époque. [...] Je venais de découvrir (l'entreprise qui a fait une demande de licenciement économique) peu de temps avant. Les faits, je crois, avec l'enquête contradictoire, remontent à début mai. Moi je suis arrivé en février sur la section, ça faisait 2 mois et demi, je ne connaissais quasiment pas l'entreprise. Ceci dit, j'avais vu passer leur plan social. Il y avait à la fois procédure de redressement judiciaire et plan social, donc bien évidemment, j'avais été forcé de m'intéresser un peu à l'entreprise, mais je ne pouvais pas dire que je la connaissais. Je ne la connaissais que par les documents du plan social, je n'avais pas une connaissance pratique, je ne m'y étais jamais rendu." (Inspecteur du travail)

"Je ne connaissais pas l'entreprise. Ce n'est pas moi qui suis cette entreprise, qui est une petite structure, et c'est un contrôleur du travail qui suit de façon plus spécifique, cette entreprise. Les connaissances que j'ai, c'est en discutant avec le contrôleur, s'il a des éléments, et puis le dossier d'entreprise. Mais dans le dossier d'entreprise, il $\mathrm{y}$ a très peu d'éléments. [...] J'ai très peu d'éléments, en fait, sur l'état des relations même sociales et du dialogue social, étant donné qu'il n'y a pas de représentant du personnel élu dans l'entreprise." (Inspecteur du travail)
\end{abstract}

En outre, la volonté farouche des inspecteurs du travail de ne pas se voir imposer de contraintes qui homogénéiseraient leur activité conduit à ce que l'activité de contrôle est fortement marquée par les contextes locaux (régulation collective plus ou moins forte) ou par la personnalité même de l'inspecteur du travail. L'ethos du professionnel peut donc être une conception individuelle autant qu'une conception partagée par l'ensemble des membres du corps.

D'autre part, le corps des inspecteurs du travail n'est pas monolithique et est touché par les modifications qui affectent d'autres groupes de salariés. Nous ne pouvons, dans les limites de cette enquête, tirer des enseignements généraux. Toutefois, l'affirmation d'un représentant d'un DRH, ne semble pas totalement dénuée de pertinence et semble reprendre des impressions que nous avons pu avoir dans les entretiens.

\begin{abstract}
"Mais aujourd'hui, et particulièrement parmi les plus jeunes, on se rend compte que les inspecteurs du travail comprennent et savent qu'il y a absolument besoin d'un dialogue social dans l'entreprise, et pour qu'il y ait dialogue social, il faut qu'il y ait un certain consensus entre les représentants syndiqués ou du personnel et la direction. Et que quand il y a des litiges qui sortent du cadre professionnel, c'est-à-dire qui rentrent dans le cadre de la fraude dans le cadre du vol, du non-respect, ils n'insistent pas et ne cherchent pas à maintenir un salarié qui est à ce stade-là dans une entreprise." (DRH, PME)
\end{abstract}

Tout se passe comme si les inspecteurs du travail les plus jeunes se positionnaient beaucoup plus en techniciens qu'en défenseurs des salariés. Une étude centrée spécifiquement sur ce corps produirait certainement des informations intéressantes qui éclaireraient de manière plus systématique que ce qui a pu être réalisé dans cette enquête, le problème des licenciements et celui de "l'effectivité du droit".

Ces différences dans la manière de concevoir le travail comme dans la manière de le réaliser constituent la marque d'une certaine subjectivité de la décision de l'inspecteur du travail. Un inspecteur du travail évoque ainsi la "subjectivité inhérente à la décision". Elle est, d'une part, le reflet d'une certaine représentation par l'inspecteur du travail de ses missions (1), elle dépend, d'autre part, de l'appréhension, par l'inspecteur du travail, du contexte (2). 
Les fonctions de l'inspecteur du travail sont prévues dans plusieurs textes. Tout d'abord, la convention $\mathrm{n}^{\circ} 81$ de l'Organisation internationale du travail (OIT) sur l'inspection du travail signée le 11 juillet $1947^{1}$ (que l'on retrouve, en substance, aux article L. 611-1 et R. 611-1 et 2 du Code du travail) qui définit, en son article 3, les missions de l'inspection du travail: contrôle de l'application de la législation du travail dans les entreprises, vérification de l'application du droit et signalement aux pouvoirs publics des situations qui nécessiteraient une évolution de la réglementation (cf. encadré).

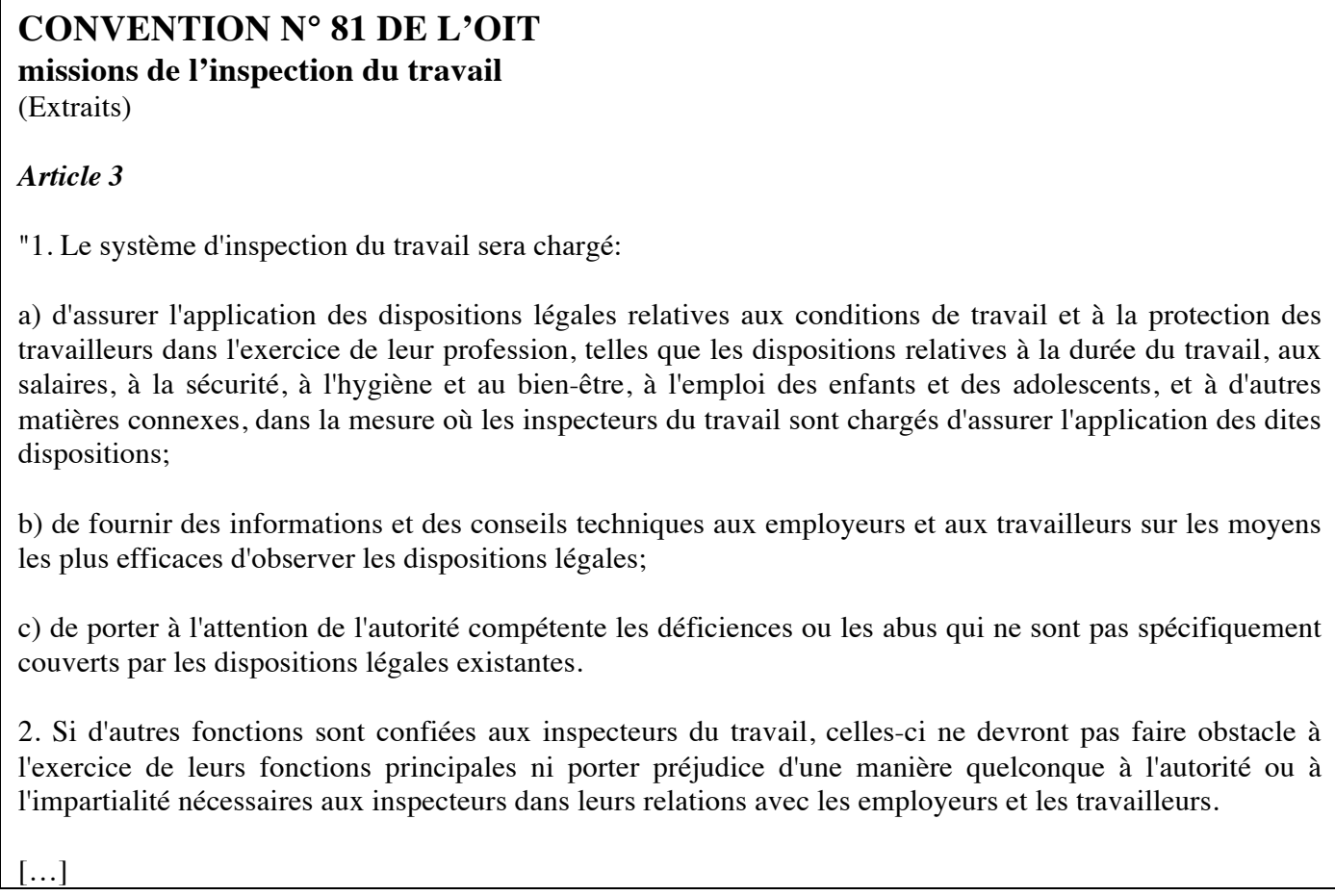

Il n'a pas été expressément prévu, dans ces missions, l'autorisation de licencier. Il est intéressant de noter que les inspecteurs du travail y étaient hostiles, ainsi que l'explique l'historien J.-P. Le Crom: "Leurs rapports du début des années cinquante montrent qu'ils répugnent à jouer un rôle qu'ils aimeraient voir tenu par la justice ou par des commissions paritaires. Beaucoup d'entre eux considèrent que leur rôle exclusif est de faire respecter les textes et que cette mission de donner leur accord ou non au licenciement nuit à leur image auprès des salariés. Certains ajoutent même qu'ils retrouveront avec crainte certains de ces salariés comme permanents syndicaux en face d'eux mais en-dehors de l'entreprise, une fois le licenciement autorisé"2.

La convention de l'OIT apparaît aujourd'hui encore extrêmement prégnante chez les inspecteurs du travail, en particulier s'agissant de son article 6 auquel ils se réfèrent souvent comme argument d'autorité face à une immixtion éventuelle dans leur dossier :

\footnotetext{
${ }^{1}$ Convention concernant l'inspection du travail dans l'industrie et le commerce entrée en vigueur le 7 avril 1950, complétée par le Protocole du 22 juin 1995 relatif à la convention sur l'inspection du travail.

${ }^{2}$ J.-P. Le Crom, op. cit., p. 96.
} 


\section{CONVENTION N'81 DE L'OIT}

L'indépendance des inspecteurs du travail

Art 6 "Le personnel de l'inspection sera composé de fonctionnaires publics dont le statut et les conditions de service leur assurent la stabilité dans leur emploi et les rendent indépendants de tout changement de gouvernement et de toute influence extérieure indue."

Un décret du 14 mars 1964 (n 64-251) les a d'ailleurs soustrait à l'autorité du préfet. Cette indépendance est vécue par les inspecteurs du travail comme un gage de qualité des missions dont ils sont investis. Elle peut ainsi constituer à la fois une limite à l'accès de tierces personnes aux dossiers en même temps qu'elle fonde en droit et légitime les pouvoirs de contrôle de l'inspecteur du travail dans l'entreprise. La frontière apparaît cependant ténue, tant au plan juridique que dans les pratiques, entre contrôle et immixtion dans la gestion de l'entreprise. Le Conseil d'Etat semble opter pour une appréciation assez large des pouvoirs de l'inspecteur du travail, en particulier lorsque la tentative de reclassement de l'intéressé s'impose (en cas de licenciement pour motif économique ou pour inaptitude). Mais à l'inverse, l'inspecteur du travail ne peut intervenir directement dans la gestion de l'entreprise, sous peine de commettre une faute lourde.

CE 29 JUIN 1990 - No N8088, MINISTRE DES AFFAIRES SOCIALES ET DE L'EMPLOI C/ SOCIETE GROUPE C.E.R.P, 1RE/4E SOUS-SECTION REUNIES.
Extrait
"le refus de l'inspecteur du travail était en réalité motivé par la volonté d'amener la société 'Groupe
C.E.R.P.' à signer avec une société concurrente, la société 'Le Centenaire', un protocole d'accord élaboré
par l'administration, visé et signé par elle, aux termes duquel les contrats de travail de 6 des salariés de
l'agence étaient repris par la société 'Le centenaire' en contrepartie du versement d'une indemnité
compensatrice au profit du nouvel employeur et du paiement des salaires du mois de juillet au personnel
concerné, le tout à la charge de la société 'Groupe C.E.R.P.' [...] l'ensemble des illégalités ainsi commises
et des agissements de l'administration susrelatés [constituant] une faute lourde qui est de nature à engager
la responsabilité de l'Etat"

Cette indépendance, sur laquelle s'est bâtie la représentation qu'ont les inspecteurs de leur travail, apparaît comme un mécanisme structurant le déroulement de la procédure (l'inspecteur du travail peut faire état de la confidentialité de ses sources). Cette indépendance trouve, au plan juridique, sa contrepartie dans la nécessaire motivation de la décision par l'inspecteur du travail ${ }^{1}$. Elle constitue un gage d'impartialité et permet un contrôle hiérarchique et juridictionnel. Il ne peut ainsi se référer à la seule gravité des faits reprochés au salarié protégé et à l'absence de lien avec le mandat, sa décision ne pouvant alors être regardée comme suffisamment motivée "dès lors qu'elle ne précise pas la nature des faits reprochés" ${ }^{2}$.

Les inspecteurs du travail font quasiment tous référence à cette indépendance du corps.

"L'inspection du travail est construite sur un principe d'indépendance, qui, si on regarde les textes, peut être comprise de manière plus réduite que notre culture commune ne l'a construite. Notre culture commune l'a construite comme étant une indépendance à la fois du choix des contrôles des entreprises que je vais voir et des suites que je donne. Bien sûr, je suis censé utiliser les voies légales pour faire ça." (Inspecteur du travail)

Cette indépendance est une garantie que le penchant du corps vers le rééquilibrage des rapports entre salariés et entreprise va continuer.

\footnotetext{
${ }^{1}$ R.436-4 du code du travail.

${ }^{2}$ CE 5 juin 1991 - $\mathrm{N}^{\circ}$ 104743, Compagnie Air Gabon c/ Dolo.
} 
"Pour moi l'indépendance se défend non pas parce que je fais ce que je veux sans rendre de compte à personne, je pense d'ailleurs qu'il faut rendre compte de ce qu'on fait, mais les premières personnes à qui on doit rendre des comptes, c'est les victimes du droit du travail. C'est-à-dire les gens qui viennent se plaindre des problèmes qu'ils ont, soit en tant que représentants du personnel, soit à titre individuel, sur leur situation." (Inspecteur du travail)

Les difficultés d'accès au terrain dans un des départements peuvent, pour partie, s'expliquer par les craintes, plus ou moins marquées, ressenties par la plupart des inspecteurs du travail de voir remettre en cause leur indépendance au travers de ce qui a pu être perçu comme une immixtion dans leurs "affaires". C'est ce qu'explique un inspecteur du travail qui, par ailleurs, ne s'est pas opposé à l'enquête de terrain.

"Il y a, de mon point de vue, une tendance à laquelle je pense que la situation politique actuelle n'est pas tout à fait pour rien, mais aussi parce qu'il y a une volonté du ministère de cadrer un petit peu l'action de l'inspection du travail. C'est dit entre les lignes, c'est un truc qui s'applique dans tous les ministères, qui est là aussi un calque de ce qui se passe dans le privé, c'est-à-dire, [...] maintenant il faut travailler sur objectif dans tous les ministères, avec des missions, des axes, etc..... Et jusqu'au plus bas de l'échelle, on donne aux gens des objectifs sur un domaine particulier, et on doit remonter ça. Sauf que quand vous êtes dans un service de contrôle de la loi, cette question-là est véritablement un problème, de mon point de vue, puisque notre objectif est écrit dans la loi : il est de faire appliquer le code du travail dans les entreprises dont j'ai la charge. Donc je n'ai pas à sous-entendre des choses que j'abandonne. On ne vous dit pas ouvertement : on intervient ou n'allez pas là, mais à partir du moment où on vous dit de plus en plus, c'est la couleur de ces stylos qu'il vous faut contrôler, comme on est jamais que 200 en France à contrôler, à l'arrivée [...], donc ça veut dire que vous ne ferez pas autre chose. Alors, ça peut paraître un étranger à la question dont je viens de parler, mais de mon point de vue, moi je le dis parce que je pense ça n'est pas anodin de voir les choses ainsi. [...] On n'est pas encore au stade ou on vous dit ouvertement : vous ne devez pas faire ceci ou cela. Mais on est déjà dans un stade où c'est dit dans les notations des gens." (Inspecteur du travail)

Le droit du travail constitue l'autre source, en même temps qu'elle la délimite, de l'intervention de l'inspecteur du travail. Or, le droit du travail s'est construit sur le constat de l'inégalité des parties. C'est ce qui justifie, par exemple, que "le doute profite au salarié"1. Cette dimension semble fortement intériorisée par les inspecteurs du travail qui se situent parfois comme le prolongement du Code du travail, ainsi que l'explique un inspecteur du travail.

"Ce qui est critiqué c'est autour de la question de l'objectivité de la place de l'inspecteur du travail. Moi je n'y crois pas vraiment, le code du travail est un droit de défense, c'est un droit de protection, vu sous cet angle, il n'est pas partial, je pense que c'est une illusion de croire en la neutralité." (Inspecteur du travail)

À la question : quel est le rôle de l'inspecteur du travail, un inspecteur du travail répond :

"Tirer, interpréter la jurisprudence".

Cette représentation qu'a l'inspecteur du travail de son rôle, modèle le sens de sa décision.

"Pour tout vous dire, depuis 3 ans, il n'y a que 2 fois où j'ai dit non malgré la volonté du salarié. Un cas où à l'évidence c'était une démission que la salariée avait demandé. Moi, j’appelle ça un faux licenciement. [...] Sa divergence c'était la nouvelle organisation. À l'évidence, elle avait envie de partir et il fallait que ce soit couvert par un licenciement. D'abord ce n'était pas

\footnotetext{
${ }^{1}$ Article L.122-14-3, dernier al., par exemple.
} 
quelqu'un qui avait été très actif, il faut dire les choses comme elles sont parce que parfois, on le prend en considération, c'est vrai. L'autre cas, c'était [l'entreprise X] [...]. Et là, j'ai fait un refus parce que les gens avaient exprimé leurs sentiments très partagés à la différence de [l'entreprise Y], c'est-à-dire qu'ils me disaient très ouvertement leurs critiques de la mesure qui était faite. À l'évidence, là aussi ils avaient l'impression, et c'était vérifié par des éléments de chiffres d'effectifs, qu'on remplissait très fortement la barque, [...] qu'on profitait [de la rénovation des lieux] pour changer le personnel." (Inspecteur du travail)

La décision se construit à partir d'éléments objectifs et subjectifs qui donnent lieu à un arbitrage de l'inspecteur du travail, en particulier dans des "cas limites", c'est-à-dire lorsque pratique et droit se heurtent, comme dans le cas de la transaction. C'est ce qu'explique un inspecteur du travail:

\begin{abstract}
"Alors plus globalement, mais là on sort du cadre de ce dossier, mais ça vous intéresse peut-être, il $\mathrm{y}$ a effectivement une vaste question qui est la suivante : à partir du moment où on a des éléments pour refuser un licenciement, normalement il faut le faire. Le Conseil d'Etat lui dit oui, vous devez refuser parce que le mandat dont est investi ce salarié le dépasse, dépasse sa propre personne de salarié, ne lui appartient pas et il est investi d'une sorte de mission collective à travers son identité de salarié, et ce n'est pas à lui de décider s'il doit partir, quitter l'entreprise. [...]. Ça m'est arrivé d'adopter cette position, et ça m'est arrivé vis-à-vis de salariés, dont je pouvais savoir, pour avoir une connaissance de l'entreprise et de leur activité, qu'en fait, ils n'avaient aucune activité, et que c'était un mandat de pure protection, et qu'à la fin, ils le monnayaient pour partir avec de meilleures conditions que des salariés qui n'avaient pas de mandat (En refusant le licenciement, vous protégez l'entreprise.) Oui, je lui fais faire des économies, mais je protège aussi, je pense, l'institution. Je pense que les rumeurs courent vite dans les entreprises, et un représentant du personnel, aussi bon qu'il ait pu l'être d'ailleurs en tant que représentant du personnel, lorsqu'il part avec sa transaction, il y aura toujours des salariés pour dire : lui a droit à une transaction mais pas nous. Ça entache toujours un peu l'institution, c'est vrai, mais en même temps si je sais qu'il a eu un rôle effectif, je pense aussi qu'il a valorisé l'institution représentative et son rôle. Mais à l'inverse, pour le salarié qui n'a joué aucun rôle et qui n'a fait que se planquer en prenant ce mandat et qu'il puisse partir en le monnayant, là effectivement, je pense que c'est une décrédibilisation totale du mandat vis-à-vis des salariés. On cherche évidemment à éviter que le rôle des délégués soit décrédibilisé à ce point. [...] A l'inverse, vous avez des cas de figure où les salariés, à l'inverse, justement parce qu'ils ont joué un rôle sont complètement flingués par leur employeur. Il y a des cas de figure stupéfiants, les téléfilms sur le harcèlement à la télé, à côté, c'est rien. Le salarié arrive, il est déjà sous traitement médical depuis des mois, il s'effondre en larmes dans votre bureau, il nous supplie quasiment à genoux de le laisser partir. Dans ces cas-là, je prends quand même en compte la réalité humaine aussi, je me dis qui suis-je pour brandir le code du travail et dire la loi rien que la loi, et vous retournerez encore en baver, quitte à ce que, pourquoi pas, il se foute en l'air. J'ai eu des cas de figure, assez rares heureusement, mais j'ai connu des cas de figure où on se demandait si le salarié allait pas se jeter par la fenêtre en sortant d'ici si je lui disais que je refusais. Et je le dis sans dramatiser mais c'est une guerre." (Inspecteur du travail)
\end{abstract}

Il est bien évident que comme toute personne, les préférences des inspecteurs du travail servent de premier prisme pour constituer leur jugement. Même s'il tente de mettre à distance ces préférences afin de porter un jugement "en toute objectivité", cela oriente la manière dont il se construit une opinion.

La plupart des inspecteurs du travail interrogés déclarent ainsi avoir pour mission de rééquilibrer le rapport de forces en faveur des salariés ; ce jugement est partagé par la plupart des gestionnaires qui sont en contact avec eux.

\footnotetext{
"Ayant moi-même des préoccupations sociales comme tout le monde, j'avais l'impression de pouvoir exercer un métier qui me permettait de penser, à tort ou à raison, mais sans grandes illusions, qu'on pouvait mettre en face certaines préoccupations sociales et une certaine activité professionnelle. Faire en sorte qu'un certain ordre public social puisse ne pas être trop bafoué, sinon parfaitement appliqué, mais éviter au moins certaines dérives." (Inspecteur du travail)
} 
Cette orientation pro salariée est assez marquée comme le traduisent deux inspecteurs du travail : le premier dans l'attention toute particulière qu'il prête au dossier, le second quand il répond à la question de l'objectivité des inspecteurs.

\footnotetext{
"Elle n'était pas d'accord, elle ne voulait pas partir. Donc, à partir de là, vous ouvrez vos oreilles.» (Inspecteur du travail)

"Ce qui est critiqué c'est autour de la question de l'objectivité de la place de l'inspecteur du travail. Moi je n'y crois pas vraiment, le code du travail est un droit de défense, c'est un droit de protection, vu sous cet angle, il n'est pas partial, je pense que c'est une illusion de croire en la neutralité." (Inspecteur du travail)
}

Ce biais est suffisamment connu et s'applique de manière identique à tous les individus, sociologues et juristes y compris, pour qu'il puisse sembler ne pas présenter beaucoup d'intérêt. Toutefois, on ne peut se permettre de l'ignorer tout à fait. En effet, les représentations idéologiques, dans son sens le plus neutre, sont au centre de l'activité de l'inspecteur du travail et de la manière dont il prend ces décisions. L'utilisation du terme "perception" dans la citation qui suit est révélatrice de l'impossibilité où se trouvent les inspecteurs du travail d'objectiver totalement leurs jugements.

\footnotetext{
"Encore une fois, c'est beaucoup la perception que je peux avoir de la volonté de l'acharnement mis par l'employeur à vouloir se débarrasser de quelqu'un et de ce que je peux sentir comme volonté de mettre à bas une section syndicale ou de rendre plus difficile la représentation du personnel dans l'entreprise." (Inspecteur du travail)
}

En effet, l'avis que donne l'inspecteur du travail est très fortement marqué par les conceptions particulières qu'il se fait du monde social.

La conception qu'il se fait de l'individu et de son autonomie apparaît déterminante.

Elle explique certaines décisions que les inspecteurs du travail ont été amenés à prendre.

\footnotetext{
"Je pense aussi que les gens sont assez grands pour faire la part des choses. (...) Je pense que les salariés sont des grandes personnes responsables. (...) On compte sur lui pour se défendre, c'est son emploi qui est en jeu. Et s'il ne veut pas se défendre, on ne va pas le sauver malgré lui." (Inspecteur du travail)
}

\section{2) L'appréhension du contexte}

Ainsi que nous l'avons déjà signalé en introduction, les faits bruts ne produisent pas mécaniquement une situation claire que l'inspecteur du travail pourrait comparer à des standards ou aux règles inscrites dans la loi. Les inspecteurs sont confrontés à une pluralité de situations auxquelles ils n'appliquent pas les mêmes processus d'élaboration de leur jugement. C'est pourquoi, ces processus doivent être regardés dans le détail en fonction du type de situations rencontrées.

La connaissance de l'entreprise, tant au niveau de ses modes de fonctionnement que du "climat général", par l'inspecteur du travail apparaît également déterminante. "C'est la justification de notre compétence", selon les termes d'un inspecteur du travail. 
Cette appréhension du contexte ${ }^{1}$ apparaît fondamentale pour la prise de décision. Ainsi que le relevait en 1974 le Procureur général Touffait, le législateur de $1946^{2}$ a entendu confier certaines missions à l'inspecteur du travail " [...] qu'il estime particulièrement qualifié, sensible aux préoccupations sociales, averti des difficultés de la direction d'une entreprise, et préparé à intervenir dans le règlement des conflits collectifs"'3.

L'inspecteur du travail cherche, lors de la prise de décision, si la réalité des éléments de faits avancés est avérée. Il utilise, pour ce faire, sa connaissance du contexte social et économique, présent et passé.

"J'étais au courant depuis longtemps des difficultés économiques de l'entreprise." (Inspecteur du travail)

Un licenciement économique apparaîtra d'autant plus fondé que les difficultés économiques de l'entreprise sont notoires. La connaissance de l'entreprise, qui s'acquiert sur la durée, permet à l'inspecteur du travail de "situer" sa décision.

"Ce qui importe pour moi, c'est [...] ce que je sais d'avance de l'entreprise, des relations sociales dans l'entreprise, de toutes les plaintes que j'ai pu avoir précédemment en termes de discrimination par la section syndicale auquel appartient le représentant du personnel." (Inspecteur du travail)

L'existence de "précédents", tels des licenciements déjà prononcés sans réelles tentatives de reclassement, des "propositions de reclassements bidons"... peuvent intervenir dans la prise de décision :

"Oui, je connais la société $\mathrm{S}$. parce qu'il y a eu de gros problèmes de sécurité : établissement Seveso, avec 3 ou 4 incendies au cours des 10 dernières années, mais aussi pour des relations sociales difficiles et ce depuis toujours. De plus, une CFDT mal organisée, et des représentants qui ne sont pas au top, ce qui ne facilite pas les choses, tant pour l'inspection du travail que pour les salariés qui y sont employés." (Inspecteur du travail)

Cette connaissance du contexte socio-économique s'acquiert par les visites réalisées dans l'entreprise, les contacts qui ont pu être établis avec un salarié protégé, les courriers reçus par l'inspection du travail, les saisines de l'inspecteur du travail par le salarié protégé, l'examen des comptes-rendus de séances de CHSCT ou CE... qui aident à reconnaître le "salarié actif", ainsi que l'explique un inspecteur du travail des Hauts-de-Seine :

"C'était la seule sur N. qui disait quelque chose [...] ; [Les dirigeants] me disaient : c'est encore elle qui vous envoie." (Inspecteur du travail)

Le passé professionnel du salarié protégé intervient alors de manière déterminante pour cette même personne

"On ne lui reprochait rien sur son travail" ; "dossier qui avait été monté trop vite en cherchant à grossir les faits. (...) Il faisait l'affaire ; il pose problème du jour où il pose des questions. (...) Tout d'un coup, il est bon à jeter." (Inspecteur du travail)

\footnotetext{
${ }^{1}$ Comparant les logiques à l'œuvre dans les jugements des tribunaux judiciaires et dans les décision des IT, l'historien du droit J.-P. Le Crom souligne, analysant les conflits des décennies 60-70, que les inspecteurs du travail, sont, lors de l'appréciation de la faute, "plus soucieux du contexte social, plus conscients aussi des habitudes ouvrières". J.-P. Le Crom, L'introuvable démocratie salariale. Le droit de la représentation du personnel dans l'entreprise (1890-2002), Paris, Syllepse, coll. Le Présent Avenir, 2003, 194 p., p. 100.

${ }^{2}$ Loi du 16 avril sur le statut des délégués ouvriers.

${ }^{3}$ Cass. Chambre mixte 21 juin 1974, Perrier, Dalloz 1974, p. 593, Concl. Touffait, p. 596.
} 
C'est finalement la présence ancienne sur un terrain donné qui confirme l'inspecteur du travail dans son rôle, lui permettant d'avoir une vision panoramique d'une affaire sur laquelle il va devoir se prononcer, comme le raconte cet inspecteur du travail.

\begin{abstract}
"(Vous avez une connaissance de l'entreprise parce que vous êtes sur la section). Certains craignent un encroûtement de la part des collègues. Moi je pense qu'au contraire, on a une meilleure connaissance des entreprises. Alors à charge d'être vigilant, de faire en sorte que s'inscrire dans la durée ne vienne pas vous paralyser, mais je pense qu'à l'inverse, il y a des atouts intéressants. Et là vous avez quand même une bonne connaissance des gens qui y sont, des évènements passés, vous pouvez vous référer à des épisodes qui datent de 5,6 ans et plus, et donc vous avez là une vision sur plusieurs années de ce qui s'est passé, et notamment du rôle des représentants du personnel. Après vous avez le dossier de l'entreprise, vous retrouvez des traces, y a $t$ il eu des courriers ? Parfois leur participation, ils ont des engagements assez forts, vous dressez un PV, ils se constituent partie civile sur votre PV, ce qui vient vous appuyer, etc... S'il y a leur rôle en interne dans l'entreprise, des salariés viennent vous voir et vous disent : "je suis défendu par un tel", enfin bref, on a quand même la possibilité de connaître un certain nombre de choses. Notamment, on peut avoir, on ne les a pas forcément, moi je demande systématiquement les comptes-rendus des réunions de CHSCT. Donc on voit bien qui intervient, qui n'intervient pas, mais on peut aussi demander les comptes-rendus de CE, qu'on n'a pas systématiquement, mais on peut les demander. Moi ça m’est arrivé, notamment sur certaines affaires de les demander sur un an, donc j'avais une dizaine de PV de CE, on voit bien là qui intervient, s'ils sont bien faits, s'ils sont détaillés. On voit bien qui intervient, qui n'intervient pas, avec quelle qualité d'intervention, quelle pugnacité aussi des fois. Et vous voyez bien aussi quels sont ceux qui viennent dans votre bureau et vous disent là il faut agir. Lorsque vous êtes en visite dans les entreprises, vous pouvez demander à être accompagné par les délégués, par les membres du CHSCT, quels sont ceux qui sont présents, qui interviennent. Vous allez vous-même aux réunions du CHSCT quand vous pouvez y aller, donc à ce moment-là, vous voyez bien aussi qui intervient, qui met en œuvre, qui s'oppose, qui prend des risques, etc..." (Inspecteur du travail)
\end{abstract}

Les inspecteurs du travail commencent ainsi à se construire une opinion bien en amont de la demande de licenciement. C'est en fait l'idée qu'ils se font des relations sociales de l'entreprise telles qu'ils les ont connues au fil du temps qui paraît déterminante. L'historique de leurs relations avec l'entreprise, avec le ou les salariés protégés, ce qu'ils connaissent des relations internes constituent autant d'indicateurs dans lesquels les inspecteurs du travail vont puiser leur argumentation.

"(On) m'avait signalé les difficultés entre l'encadrement de cette entreprise et ce directeur qui a une façon assez personnelle de gérer son entreprise et qui à priori ne donnait pas suffisamment de responsabilités à son personnel d'encadrement." (Inspecteur du travail)

"Ce qui importe pour moi, c'est beaucoup plus ce que je sais d'avance de l'entreprise, des relations sociales dans l'entreprise, de toutes les plaintes que j'ai pu avoir précédemment en termes de discrimination par la section syndicale auquel appartient le représentant du personnel. Ou des choses qui me sont révélées à l'occasion de l'enquête." (Inspecteur du travail)

Il ne s'agit pas d'une connaissance "livresque" construite uniquement à partir des échanges écrits entre l'inspecteur du travail et ses interlocuteurs ou à partir des seules informations produites par l'entreprise et par le salarié. Elle fait aussi appel aux sensations, à la compréhension en situation, en quelque sorte physique, que peuvent se construire les visiteurs d'une entreprise, ce que manifeste en creux la citation ci-dessous.

"Je ne pouvais pas dire que je la connaissais. Je ne la connaissais que par les documents du plan social, je n'avais pas une connaissance pratique, je ne m'y étais jamais rendu." (Inspecteur du travail) 
L'impression générale paraît ainsi déterminante.

"L'enquête contradictoire a été relativement rapide pour la plupart d'entre eux. [...] Il y avait quelque chose un peu "sauve qui peut". [...] Ce qui m'était apparu assez rapidement quand même, c'est qu'il y avait un assez mauvais climat dans l'entreprise et beaucoup de salariés ne croyaient pas aux perspectives de l'entreprise pour le futur. [...] Puis il y a le climat interne aussi, c'est-àdire qu'on y croit de moins en moins, on voit partir des gens en démission. On sent que les gens présents cherchent à l'extérieur parce que évidemment les bruits et les récits circulent beaucoup dans l'entreprise, c'est un peu tous ces indices. C'est impalpable mais c'est ainsi que les gens en parlent, et autant de petits indices comme ça qui, réunis, font un peu faisceau et vous laissent penser que bon, on a l'impression que c'est un peu sauve qui peut. " (Inspecteur du travail)

Bien sûr, le regard que les inspecteurs du travail portent sur le licenciement est très marqué par le jugement qu'ils portent sur l'entreprise, jugement lui-même marqué encore une fois par l'idéologie personnelle, c'est-à-dire par une conception de la manière dont les entreprises devraient traiter leur personnel.

"(L'inspecteur du travail) a bien compris qu'il ne pouvait pas nous coincer sur les licenciements
parce que les salariés poussaient : quand est ce que tu nous vires, etc... Donc, il a essayé de nous
attaquer sur les fondements même du plan social, qu'il n'était pas crédible, etc..., en allant assez
loin. [...] Je pense qu'il en veut à (l'entreprise) parce que (l'entreprise) est un gros mythe (dans la
ville) : c'est le plus gros établissement, il nous le rappelle à chaque fois, c'est une boîte américaine
qui a en plus une bonne image (dans la ville). Donc pour un inspecteur du travail qui est très
rigoureux par rapport à une approche juridique française, etc... Voir qu'une boîte peut fonctionner
à l'américaine, en plus que les gens soient contents ça doit être un peu agaçant, [...] Je pense que
pour lui, les gens se font avoir, se font manipuler, se font marcher dessus, enfin ce genre de
choses.» (DRH, grande entreprise)

En outre, l'inspecteur du travail a souvent une vision tronquée des entreprises dont il a le contrôle, car ce sont surtout les aspects négatifs qui remontent à son niveau.

"Je vois les mécontents par définition, je ne vois pas ceux qui sont contents." (Inspecteur du travail)

Mais l'inspecteur du travail évalue aussi l'orientation des actions engagées par l'entreprise et ses effets sur le fait syndical. Il effectue cette évaluation sur un double registre : celui des "flux" et du "stock".

Pour le "flux", l'inspecteur du travail s'occupe du licenciement et se demande s'il est dirigé contre le mandat. Ce sont donc les seuls objectifs de l'entreprise qu'il confronte aux formes du licenciement ; l'historique des relations entreprise - représentants du personnel est également interrogée. De ce fait, l'acceptation ou le refus du licenciement peuvent aller à l'encontre des souhaits des salariés.

Mais l'inspecteur du travail juge aussi l'état du "stock" syndical. La permanence du fait syndical après les départs des salariés protégés constitue parfois quasiment une condition sine qua non de l'autorisation de licenciement.

L'inspecteur du travail est ainsi amené, pour reprendre les termes de l'un d'eux, à se faire "une opinion", il "habille" ensuite. Un inspecteur du travail justifie cette position par le fait que

"Les rapports sociaux, c'est autre chose que du droit"." (Inspecteur du travail) 
Le travail de justification des décisions vient alors formaliser en partie la manière dont ils se sont construits leur jugement. Si, dans les cas non-litigieux, l'argumentation peut être réduite à la portion congrue (parce que l'inspecteur du travail pense que l'affaire s'arrête avec sa décision), il n'en va pas de même dans les autres cas. Si l'inspecteur du travail soupçonne qu'un recours sera déposé suite à sa décision, il va procéder à une construction "défensive" qui vise à garantir qu'il ne sera pas désavoué.

C'est en partie pour cela que les salariés protégés ne sont pas crus sur parole et qu'on leur demande de prouver d'une manière ou d'une autre, la version qu'ils présentent.

"On lui demande d'étayer aussi. Vous le mettez au pied du mur aussi en lui disant : très bien, le lien avec le mandat, je veux bien, mais dîtes moi en quoi, montrez-moi, vous avez fait cette demande quand, vous avez des courriers, des témoins, pourquoi pas ? Voilà, tout élément peut être valable, mais on cherche aussi à avoir des éléments objectifs." (Inspecteur du travail)

Toutefois, les inspecteurs du travail rencontrés disposent d'une marge d'appréciation importante. Ils tirent ou interprètent les textes et la jurisprudence souvent en faveur des salariés, en fonction de leurs préférences idéologiques, mais aussi pour tenir compte de l'inégalité de nature du rapport salarial.

"Il est sûr qu'on prend des décisions illégales. [...] Dans ces cas-là, ce que je m'efforce de faire
vis-à-vis des membres des représentants du personnel qui restent dans l'entreprise, et vis-à-vis
aussi de l'employeur essentiellement, c'est de montrer au moins que je ne suis pas dupe.
J'autorise, mais je dis que j'avais en fait tous les éléments pour refuser. [...] Donc ce n'est pas la
peine de faire semblant de mettre des éléments d'autorisation, donc je mets des motifs
explicitement de refus, mais je dis à la fin, considérant que le salarié n'a plus aucune perspective
dans l'entreprise, autorise son licenciement. Donc il faut bien dire que manifestement, son horizon
est complètement bouché, il est grillé, que c'est lui qui souhaite partir, mais qu'en même temps, je
sais bien qu'il n'y a pas de motif économique, qu'on n'a pas fait les efforts de reclassement
suffisants, ou que la faute n'existe pas, etc... Ne serait- ce que pour dire qu'on est pas dupe mais
c'est uniquement parce que le salarié voulait partir." (Inspecteur du travail)

En outre les décisions rendues se suivent: elles ont de grandes chances d'aller dans le même sens que les décisions précédentes. Les décisions favorables peuvent ainsi être conçues, pour le salarié protégé, comme autant d'acquis qui faciliteront les prochaines décisions.

"Mon licenciement a été refusé. Donc il n'a pas aimé, ça veut dire que je réintégrais la société. [...] Là, ils ont posé un recours. [...] La direction départementale a donc refusé, je suis réintégré, ils entament un recours, donc je suis à nouveau convoqué par la personne qui était au-dessus de (l'inspecteur du travail). Ce monsieur [...] a confirmé l'avis de (l'inspecteur du travail). [...] Puis il $\mathrm{y}$ a eu un procès et la direction a été condamnée pour harcèlement moral et discrimination syndicale. [...] Je me suis dit maintenant s'il veut me virer avec son plan social, il va avoir beaucoup de mal.» (Salarié protégé, délégué syndical, membre CHSCT, syndiqué, ouvrier, 44 ans, CAP, demande de licenciement pour faute)

Il est certain que la connaissance du terrain, et notamment d'éventuels enjeux politiques du dossier, pèse aussi dans la prise de décision.

"Sur les décisions que je sais tendues, je sais très bien qu'il y aura un recours derrière, donc c'est pour ça qu'il ne faut pas hésiter à prendre un peu de temps pour faire cette enquête, parce que ça ne sert à rien de faire une enquête en allant à une décision de refus rapidement si on n'a pas assez blindé le dossier pour qu'il tienne un recours." (Inspecteur du travail)

La connaissance permet de mettre à jour le lien entre la demande de licenciement et la discrimination. 


\begin{abstract}
"Encore une fois, c'est beaucoup la perception que je peux avoir de la volonté de l'acharnement mis par l'employeur à vouloir se débarrasser de quelqu'un et de ce que je peux sentir comme volonté de mettre à bas une section syndicale ou de rendre plus difficile la représentation du personnel dans l'entreprise. Ça on le sent assez fortement, surtout si on a eu, comme je le disais, des antécédents. Si c'est la même personne qui est déjà venue nous voir pour se plaindre de l'attitude de son employeur vis-à-vis de lui et 3 mois plus tard, l'employeur me fait une demande d'autorisation de licenciement à l'encontre de cette même personne, j'essaie d'en savoir plus et de démêler entre l'appréciation des faits tels qu'ils me sont rapportés de façon brute par l'employeur, et puis ce qui peut être derrière comme lien avec le mandat. Ça pour moi, c'est important, c'est toute la survie de la représentation de personnel dans les entreprises et la relève qui peut être faite par les autres aussi, parce que quand des salariés voient qu'un de leurs représentants s'est fait licencier, alors qu'ils ont eux-mêmes la perception que c'est injuste, que l'employeur a profité de l'occasion, c'est d'autant plus difficile pour d'autres de prendre la relève." (Inspecteur du travail)
\end{abstract}

Cette connaissance du contexte, issue de la présence, apporte aussi la crédibilité ou l'absence de crédibilité et, le cas échéant, une certaine reconnaissance, comme l'explique cet inspecteur du travail dans une phase de l'entretien qui aborde plusieurs facettes des relations que les inspecteurs du travail peuvent avoir avec des dirigeants d'entreprise.

\begin{abstract}
"Quand on conseille l'employeur, on n'est pas toujours perçu comme le gendarme, mais on est parfois perçu comme celui qui a acquis une certaine expérience, avec qui parfois, on s'est confronté sur d'autres chantiers. Et on en a tiré, parfois, des leçons en se disant ce que me propose ce gars, ce n'est pas toujours si con que ça. Et ce qui me propose, ce n'est pas simplement comme un gendarme, c'est en tenant compte de son expérience, de ce qu'il a appris, de la connaissance du métier, de la connaissance des techniques. Quand il ne vous connaît pas, vous apparaissez souvent comme ce que les médias ou ce que la rumeur publique présente : l'inspecteur du travail, c'est un gendarme, et le gendarme, vous ne l'aimez pas, vous ne l'aimez pas parce que vous le voyez comme celui qui verbalise, comme celui qui vous empêche de travailler quand vous en avez envie et comme vous en avez envie. Mais quand vous avez affaire à un employeur qui vous connaît depuis longtemps, ce genre de discussion ne va pas avoir lieu, parce qu'il va vous accorder une certaine confiance, une certaine autorité, une certaine crédibilité en disant il ne me raconte pas d'histoires, parce que vous savez, la rumeur vous précède. Ils vont se dire : celuilà, il connaît, ce n'est pas la peine d'essayer de lui faire croire de midi à quatorze heures, donc on va changer de terrain de discussion, et éventuellement même d'affrontement ou de travail. Il va dire : $O K$, il connaît son histoire, il connaît son boulot, il connaît la réglementation, donc on ne discute pas là-dessus. On va discuter éventuellement en prévention, on va discuter solutions de reclassement, c'est une autre façon d'aborder les problèmes. Et ça, vous ne pouvez les aborder que si, effectivement, vous avez une certaine expérience, mais aussi, en fonction de votre interlocuteur et de la connaissance qu'il a de vous. Sinon, il va discuter vos connaissances, vos compétences, etc... Et comme l'enfant avec ses parents, il y a un certain jeu, vous avez besoin d'une certaine autorité, et cette autorité ce n'est pas simplement votre titre d'inspecteur du travail, c'est aussi le crédit que vous avez acquis sur une place, dans un secteur géographique, dans une certaine profession." (Inspecteur du travail)
\end{abstract}

Au final, cette "proximité" change aussi la nature des relations inspecteur du travail / entreprise.

"Je faisais une enquête restaurant chez XXX qui licenciait un salarié pour faute. Tout au long de l'enquête, le directeur me dit : je lui reproche ça, en fait de ne pas avoir lavé le camion. Je lui réponds, est ce que vous l'avez vu par exemple, toute la journée et que pendant toute cette journée, vous ne l'avez pas vu laver le camion. Il me répond non, alors comment savez-vous qu'il ne l'a pas lavé ? il me répond qu'il a vu une tâche de sauce tomate. D'accord, mais vous ne l'avez pas vu donc vous ne pouvez pas affirmer qu'il n'a pas lavé le camion, il répond non. Alors je dis peut-être qu'il a lavé le camion mais mal. Il répond c'est vrai. Je demande à quelle heure il doit laver le camion, il répond à 14 heures après le repas, alors je demande à quelle heure avez-vous constaté la tâche de sauce tomate ? Il répond le lendemain matin, alors je demande entre $14 \mathrm{~h}$ et le matin, il n'y a pas de transport d'aliments ? Il répond que oui, il y a des transports pour le repas du soir. Donc je dis qu'on a pu tacher le camion lors des transports des repas du soir, il répond oui, mais ce soir-là, il 
n'y avait pas de produits avec de la sauce tomate. Alors je demande si on n'avait pas pu ramener des repas de midi dans lesquels il resterait de la sauce tomate? Il répond ah oui. Alors je demande si on lave le camion avant de revenir au siège ou est ce qu'on revient d'abord au siège et on lave le camion après, il répond qu'on lave le camion à la fin du repas et quand il est tout propre, on revient au siège et on ramène ce qui peut rester, etc... Toutes des questions aussi simples que ça, et j'ai conduit l'employeur à me dire en fait que tout ce qu'il reprochait à l'employé, c'étaient des déductions et non pas des constatations. Donc je lui ai dit qu'il y avait bénéfice du doute pour le salarié. Et je lui ai proposé en fait, d'éviter d'être désavoué, donc pour lui, il était peut-être plus simple de retirer sa demande que d'attendre que je prenne une décision. Alors il me dit ça veut dire que vous allez me refuser. Je lui ai répondu avec toutes les questions que je vous ai posées, avec toutes les impasses sur lesquelles je vous ai conduit, vous pensez quoi ? Il répond que vous allez me le refuser. Alors je lui ai dit que le plus simple pour lui c'etait de retirer sa demande et de prendre une sanction, éventuellement de mise à pied, sur laquelle je n'ai pas à me prononcer, plutôt que de recevoir une décision refusant son licenciement et que le salarié va brandir demain en disant : voyez, le patron voulait me licencier, il est désavoué, et c'est lui [le patron] qui perdrait la face." (Inspecteur du travail)

Certains inspecteurs du travail utilisent cette connaissance du terrain afin d'élaborer de véritables stratégies obéissant à une certaine représentation de leur rôle.

\footnotetext{
"Je pense qu'il faut qu'on soit capable, mais là, c'est un autre débat, ce n'est plus l'autorisation administrative ou le refus d'autorisation, c'est que si les gens ne supportent plus parce qu'ils ne sont pas en situation d'exercer leur mandat de délégué, il faut les protéger autrement y compris en mettant des PV, en menant des enquêtes pour délit d'entrave, etc... C'est un cadre contraignant, je sais qu'à un moment donné, je suis obligé de faire passer ce que je ramasse dans mes filets dans des mailles qui ont tel diamètre et qu'il faut présenter le bébé de telle façon, c'est tout. À la fois je ne m'affranchis pas de ça parce que je sais que je ne peux pas prendre de décisions qui ne tiennent pas compte de ça sinon, on flambe, et en même temps, je ne suis pas complètement polarisé làdessus, parce qu'à l'occasion d'une enquête, par exemple, la révélation d'une situation de délit, je ne m'interdis jamais personnellement, bien que ça fasse débat dans le corps, de relever le PV. Ça m'est déjà arrivé de sortir d'une enquête en disant: j'autorise le licenciement ou je refuse le licenciement, et je vous mets un PV pour délit d'entrave, etc... Parce que j'ai constaté dans l'enquête qu'il y avait des problèmes. Évidemment, ça ne calme pas les choses, mais quand on n'a plus moyen de faire autrement, c'est ce qu'il faut faire." (Inspecteur du travail)
}

C'est l'idée que l'on retrouve exprimée par un autre inspecteur du travail qui développe de véritables tactiques dans lesquelles les critères d'opportunité sont premiers :

\footnotetext{
"Je m'étais dit que s'il y avait une difficulté autour du volontariat [suite au plan social], à savoir protéger des gens qu'on force à être licenciés, peut-être aussi pour préparer la suite des évènements, il était intéressant que je me saisisse de cette opportunité comme d'une fenêtre de tir, qui est le seul moment et la manière la plus claire dans laquelle l'inspecteur du travail peut venir dans le débat d'un licenciement." (Inspecteur du travail)
}

Toutefois, les relations entre inspecteur du travail et salariés protégés ne vont pas toujours de soi. Par exemple dans la même entreprise, on peut avoir deux perceptions opposées du même inspecteur du travail.

"C'est un garçon (l'inspecteur du travail), je ne l'ai vu qu'une fois, mais comme tous les inspecteurs du travail, on le sent hargneux." (PDG, PME)

"Ce dont je suis sûr, c'est que (l'inspecteur du travail) n'est pas un type qui cherche à défendre les salariés.» (Salarié protégé, membre du comité d'entreprise, délégué du personnel, syndiqué, 37 ans, cadre, Bac+2, PME, demande de licenciement pour faute) 


\section{3) Les appréciations se fondent sur l'interrelation}

Les positions de chacun, la reconnaissance ou non de la légitimité, de la compétence, ou plus généralement de la valeur sociale de l'interlocuteur, a pour effet de s'en faire soit un allié, soit un ennemi.

C'est la création d'une inimitié qui nous est racontée ci-dessous. C'est la capacité supposée du salarié protégé d'intervenir dans le domaine d'expertise de l'inspecteur du travail et de lui dicter ce qu'il doit faire qui cristallise cette inimitié.

"La $1^{\text {ère }}$ fois qu'on a rencontré (l'inspection du travail), je ne vais pas dire qu'on avait un énorme savoir, mais on avait une idée théorique sur le fonctionnement du comité d'entreprise et de comment fonctionne l'inspecteur du travail. Donc on est arrivé là fort de notre savoir. [...] Donc on lui a dit: "on est venu vous voir pour que les choses avancent, pour que les salariés ne soient plus grugés, que ce soit les congés de fractionnement, les objectifs". Enfin, on lui dit tout, on lui fait la liste détaillée, et il reconnaît qu'il y a d'énormes problèmes. Mais dès qu'on lui a demandé son aide, il nous a dit: "ça on ne peut pas, ça on ne peut pas". Ne serait-ce que d'aller au siège social et d'aller voir le tableau, de constater qu'il n'y a rien. Sa réponse est : "je ne peux rien, ça ne servira à rien, ni à vous". Deuxième sujet, le CHSCT, il nous dit que ce n'était pas possible parce qu'on n'avait pas suffisamment de salariés, on lui a démontré qu'on était une entité et qu'on pouvait tout à fait, et je lui explique qu'il y a eu là un accident, là du harcèlement moral, là du harcèlement sexuel, ça ne peut pas continuer, même pour le bien de (l'entreprise). Si la justice s'en mêle, c'est (le directeur) qui va supporter tout. Il prend son code du travail, on est resté un quart d'heure, et il dit non, ce n'est pas possible. À chaque fois qu'il me donne un argument contre, moi je lui amène un autre argument et je lui sors mon code du travail en lui disant qu'il y a tel autre argument, pendant un quart d'heure, on s'est battu sur des textes juridiques parce que j'étais balaise à l'époque, à tel point qu'il me dit, ça ne me sert à rien de me battre, je l'ai fait 2 fois dans ce département, chaque fois la justice donne raison aux sociétés. Le département où je suis c'est un département patronal où régulièrement on perd ce que l'on fait. Alors je lui dis : attendez, vous êtes inspecteur du travail, vous avez eu quelques échecs, vous ne pouvez pas dire que parce qu'on n'a pas les tribunaux ou les magistrats avec nous, on ne peut pas avancer, je ne peux pas me contenter d'une réponse comme ça. Alors il me dit : c'est pourtant comme ça. Alors je réponds ce qu'on peut faire, c'est peut-être mener une enquête dans les magasins, ça vous pouvez le faire, je ne vous demande pas de régler le problème du CHSCT, mais vous pouvez envoyer au moins des inspecteurs voir ce qui se passe dans les magasins parce qu'il y a des endroits où c'est très grave : il y a la plate-forme de C. où il y a de l'exploitation de main-d'œuvre, interrogez-vous sur le harcèlement moral et sexuel, il y a des gens qui sont détruits à vie, j'ai tous les dossiers avec des courriers de médecin. Il me dit : "les problèmes que vous avez avec votre employeur, je ne vais pas à chaque fois les régler moi-même". Je lui ai dit : "Monsieur [...], je ne vous demande pas de régler les problèmes de mon employeur, on les règle tous les jours, on essaie. On n'est pas venu vous demander de régler nos problèmes, on est venu vous demander d'intervenir." (Salarié protégé, délégué syndical, cadre, 42 ans, Bac, PME, demande de licenciement pour faute)

Dès lors, ce premier contact est prédictif de ce que vont être les suivants, entachés de soupçons.

"Au départ, c'est avec (l'autre salarié protégé) que ça ne s'est bien pas bien passé. Parce que quand on est arrivé, la $1^{\text {ère }}$ fois qu'on a vu (l'inspection du travail) [...] Donc on est parti sur des relations qui n'étaient pas excellentes, et très honnêtement, humainement, c'est un mec que je ne sens pas bien, j'ai du mal à discuter avec lui, je ne sais pas où il veut en venir. Je le trouve froid, et j'ai l'impression qu'il a un a priori sur (mon collègue) et moi. Est ce qu'il nous prend pour des illuminés, pour des fauteurs de troubles, mais j'ai l'impression qu'il ne porte pas beaucoup de crédits à ce qu'on raconte. Et qu'il porterait plus facilement de crédits à ce que raconte la direction. Je ne veux pas dire qu'il travaille pour la direction mais quand la direction apporte des arguments, et nous les nôtres, il entend plus facilement ceux de la direction que nos arguments à nous. Il aurait tendance à les remettre en cause, ceux de la direction, non. Quoi que dise la direction, quand elle lui apporte des chiffres, il ne les conteste pas, alors que nous on sait pertinemment que pendant 2 
ans, on s'est battu parce qu'on savait très bien qu'elle nous mentait sur certains trucs. [...] Je pense que (le DRH) se l'est mis dans la poche.» (Salarié protégé, membre du comité d'entreprise, délégué du personnel, syndiqué, 37 ans, cadre, Bac+2, PME, demande de licenciement pour faute)

\begin{abstract}
"Alors je lui dis : "moi il y a une chose que je ne comprends pas [...] c'est qu'à chaque fois que je vous dis quelque chose, vous le vérifiez, vous le re-vérifiez, vous le re-re-re-vérifiez.Vous allez voir (le DRH), il vous donne une pièce ou sa parole et ça passe. Pourquoi ? Vous n'êtes pas objectif. Moi je vous donne un document écrit, lui, sa parole vous suffit. Ça ne peut pas fonctionner comme ça." (Salarié protégé, délégué syndical, cadre, 42 ans, Bac, PME, demande de licenciement pour faute)
\end{abstract}

\begin{abstract}
"Des pièces ont été remises aux uns et aux autres, et (le DRH) a fourni des pièces qu'il ne m'a jamais fourni à moi, des études de marché, des PV de comité d'entreprise, des trucs qu'on avait jamais vus. Je lui ai fait remarquer (à l'inspecteur du travail), je lui ai dit que ce n'était pas très loyal, il m'a dit qu'il me les donnerait après, mais j'aurais dû les avoir avant. Toutes les choses qu'il m'a reprochées, je lui ai dit: avant la fin de la semaine, je vous envoie un fax et je vous démontre que tout ça c'est faux". Je lui envoie donc ce fax en lui disant : "juridiquement, vous êtes à côté." [...] Parce qu'un jour au téléphone, (il) m'a dit que compte-tenu des éléments qu'il avait, il allait accepter mon licenciement, je lui ai expliqué qu'il était toujours du côté de (de l'entreprise), et même au niveau juridique, un exemple : (Le DRH) m'a convoqué pendant ma semaine de congés payés pour un entretien préalable au licenciement, il convoque le comité d'entreprise le même jour, je lui ai dit qu'on n'avait pas le droit au niveau de la procédure légale de faire ça. Donc rien que ça il aurait dû le refuser, il me répond que si, alors je lui ai dit que je l'attaquerais.» (Salarié protégé, délégué syndical, cadre, 42 ans, Bac, PME, demande de licenciement pour faute)
\end{abstract}

Signalons toutefois que l'on ne peut établir de relation entre l'état des relations ici et la décision de l'inspecteur du travail puisque le licenciement a été refusé.

\title{
La frontière apparaît parfois ténue entre le "tiers institutionnel" et la "tierce partie"
}

\begin{abstract}
"Notre échange était autant tactique que juridique dès lors que les représentants du personnel sont demandeurs. [...] L'échange est fonction de la demande. [...] À titre d'exemple, dire [aux représentants du personnel] : surveiller les incohérences. Par exemple l'entreprise continue à recruter pendant qu'elle fait des licenciements. En leur disant ça, je n'avais pas besoin de tricher avec le secret professionnel puisque ces éléments-là, ils pouvaient les avoir." (Inspecteur du travail)
\end{abstract}

Mais ces interventions "poussées" d'inspecteurs du travail ne sont-elles pas le fait d'une certaine carence du comité d'entreprise et des organisations syndicales ?

\section{II- Des interventions périphériques annexes}

L'intervention du comité d'entreprise, dont l'avis est obligatoire, n'est pas déterminante dans l'issue du conflit (A). Quant à l'organisation syndicale, son rôle est plutôt annexe (B).

\section{A) Le comité d'entreprise}

Si l'avis du comité d'entreprise est obligatoire (1), on constate qu'en pratique, il pèse peu sur l'issue de la procédure (2) 


\section{1) Un avis obligatoire}

Le comité d'entreprise est, en vertu des dispositions du Code du travail ${ }^{1}$, obligatoirement consulté sur le projet de licenciement d'un élu. Il existe deux exceptions à la règle : en cas d'absence de comité d'entreprise (article R. 436-10) ou si le salarié protégé ne remplit que les seules fonctions de délégué syndical (article L. 412-18). La loi ne donne aucune indication sur le délai de convocation du comité d'entreprise.

CODE DU TRAVAIL
(Partie Réglementaire - Décrets en Conseil d'Etat)
Chapitre 6 : Licenciement des représentants du personnel, des représentants syndicaux et des salariés
assimilés
Article R436-10
(inséré par Décret $n^{\circ}$ 83-470 du 8 juin 1983 art. 17 Journal Officiel du 11 juin 1983)
Lorsqu'une entreprise n'a pas ou n'a plus de comité d'entreprise, la demande d'autorisation de licenciement
concernant les salariés protégés définis aux articles L. 425-1 et L. 436-1 est, postérieurement à l'entretien
prévu à l'article L. 122-14, directement soumise à l'inspecteur du travail dont dépend l'établissement, par
lettre recommandée avec demande d'avis de réception.
Cette demande énonce les motifs du licenciement envisagé. Elle donne lieu à l'application des dispositions
des articles R. 436-4 à R. 436-8.

La jurisprudence a précisément défini les conditions de sa mise en œuvre. Le salarié doit être convoqué et entendu ${ }^{2}$; l'employeur doit procéder à cette formalité après l'entretien préalable. La consultation doit être loyale et contradictoire. Le comité d'entreprise se prononce par avis au scrutin secret, le cas échéant sur chacun des salariés concernés (et non dans un avis global) : il doit y avoir autant d'auditions et de votes qu'il y a de salariés protégés concernés ${ }^{3}$. Le président du comité d'entreprise, l'employeur ou son représentant, n'a pas à prendre part au vote . $^{4}$

L'avis du comité d'entreprise constitue une formalité préalable obligatoire : son omission conduit au rejet de la demande de licenciement ${ }^{5}$; elle doit être observée par l'employeur préalablement au licenciement. L'accomplissement de cette formalité après le licenciement ne peut valider rétroactivement celui-ci ${ }^{6}$.

\section{Conseil d'Etat 18 octobre 1991, $\mathbf{N}^{\circ}$ 83934, Publié aux Tables du Recueil Lebon \\ Extraits}

Vu la requête sommaire et le mémoire complémentaire enregistrés les 22 décembre 1986 et 17 avril 1987 au secrétariat du Contentieux du Conseil d'Etat, présentés pour M. Youssoufou KABA, demeurant 42 boulevard de la Villette à Paris (75019), et tendant à ce que le Conseil d'Etat :

$[\ldots]$

Considérant que les salariés légalement investis d'un mandat de délégué du personnel bénéficient, dans l'intérêt de l'ensemble des travailleurs qu'ils représentent, d'une protection exceptionnelle en vertu

\footnotetext{
${ }^{1}$ Art. L. 425-1, $1^{\text {er }}$ alinéa pour les DP ; L. 436-1, $1^{\text {er }}$ alinéa pour les membres du CE.

${ }^{2}$ CE 18 oct. 1991, Kaba, Rec. T, p. 1229, RJS 12/91, n 1340.

${ }^{3}$ CE 2 fév. 1996, RJS 3/96 n 300.

${ }^{4}$ Cass. Soc. 27 février 1985 - N 120 : "Qu'en statuant ainsi, alors que l'autorisation demandée au comité d'entreprise par le chef d'entreprise en vue de procéder au licenciement d'un salarié protégé constitue une question sur laquelle les membres élus du comité avaient seuls à se prononcer, en tant que délégation du personnel, et qu'il résulte des constatations de l'arrêt que la participation du représentant du chef d'entreprise, président du comité, avait eu pour effet de fausser les résultats du vote, la Cour d'appel a violé le texte susvisé".

${ }^{5}$ CE 11 mars 1988, Sorres, req. $\mathrm{N}^{\circ} 73.067$.

${ }^{6}$ Cass. Soc. 12 mars $1987-\mathrm{N}^{\circ} 148$.
} 


\begin{abstract}
notamment des dispositions de l'article L.425-1 du code du travail qui dispose : "Tout licenciement envisagé par l'employeur d'un délégué du personnel, titulaire ou suppléant, est obligatoirement soumis au comité d'entreprise qui donne un avis sur le projet de licenciement" ; que l'article R.436-2 du code du travail impose que cet avis soit exprimé après audition de l'intéressé ;

Considérant que M. KABA , employé par la société Armand Colin depuis 1979, a été élu délégué du personnel suppléant le 10 avril 1984 ; qu'il a fait l'objet d'une procédure de licenciement pour motif économique au même titre que 18 autres salariés ; que celle-ci s'est traduite par la réunion extraordinaire du comité d'entreprise le 12 novembre 1984 à laquelle M. KABA n'a pas assisté ; qu'il ne ressort pas des pièces du dossier et que la société Armand Colin n'apporte pas d'éléments suffisants pour prouver que M. KABA a été effectivement convoqué à cette réunion; qu'ainsi une formalité substantielle n'a pas été respectée même si l'avis rendu par le comité d'entreprise a été défavorable au licenciement ; que, dès lors, M. KABA est fondé à soutenir que c'est à tort que, par le jugement attaqué, le tribunal administratif de Paris a rejeté sa demande dirigée contre la décision du 8 janvier 1985 par laquelle l'inspecteur du travail de la 5ème section de Paris autorisait son licenciement ;

DECIDE :

Article 1er : Le jugement du tribunal administratif de Paris en date du 30 avril 1986 et la décision de l'inspecteur du travail de la 5ème section de Paris en date du 8 janvier 1985 sont annulés.

Article 2 : La présente décision sera notifiée à M. Youssoufou KABA, à la société Armand Colin et au ministre du travail, de l'emploi et de la formation professionnelle.
\end{abstract}

L'inspecteur du travail doit être en possession, préalablement à la prise de décision, de l'avis du comité d'entreprise qui ne le lie cependant pas $^{1}$ : il rend un avis obligatoire mais non conforme.

\title{
2) Un avis qui pèse peu sur l'issue de la procédure
}

En pratique, l'inspecteur du travail ne s'appuie que très peu, dans sa prise de décision, sur le comité d'entreprise. S'il vérifie et impose le respect d'une formalité substantielle, un tel avis n'est pas déterminant dans la prise de décision.

En fait, l'avis du comité d'entreprise est, tout d'abord, largement prévisible. L'avis défavorable est la règle et l'avis favorable l'exception. L'analyse des dossiers de l'inspection du travail fait apparaître que c'est d'abord la régularité des procédures qui est jugée. Sur le fond, les avis sont souvent déjà formés avant la réunion du comité d'entreprise.

Par contre, l'avis favorable donné par le comité d'entreprise semble être un critère déterminant dans l'acceptation du licenciement par l'inspecteur du travail, sans qu'on puisse savoir si l'acceptation se fait sur le caractère flagrant des reproches que le comité d'entreprise ne fait que marquer ou si c'est l'avis lui-même qui produit l'acceptation par l'inspecteur du travail.

Les entretiens réalisés avec les inspecteurs du travail montrent, d'autre part, que, de leur point de vue, l'avis du comité d'entreprise n'est parfois guère représentatif de la réalité et n'apporte rien à la prise de décision. Ils mettent également en avant la contingence de cet avis.

"Je relativise l'avis du comité d'entreprise en fonction du contexte" (Inspecteur du travail)

L'inspecteur du travail peut en effet avoir affaire à des comité d'entreprise dont les candidatures ont été "suscitées" par l'employeur, ou encore marqués par des dissensions syndicales. Dans ce dernier cas, l'avis donné par le comité d'entreprise sert aussi à régler des

\footnotetext{
${ }^{1}$ CE 6 mai 1996, Stradal industries, $n^{\circ} 169573$.
} 
comptes. Au final, il apparaît que les comités d'entreprise, du point de vue de certains inspecteurs du travail, ne remplissent pas leur fonction et leur rôle de contre-pouvoir.

\begin{abstract}
"Je ne me sens absolument pas lié par ce que le comité d'entreprise a fait compte-tenu [...] de l'absence de culture syndicale ou de culture collective qu'on rencontre dans beaucoup de Comités d'établissement. Malheureusement, donc, je ne peux pas me sentir lié et en plus il faudrait un comité d'entreprise extrêmement malin et qui ait extrêmement bien étayé le dossier pour qu'on puisse utiliser vraiment les éléments qu'il y a eu dans les débats du comité d'entreprise. Ce qui arrive de temps en temps; ce sont les plus gros licenciements économiques et ces malins savent nous procurer du biscuit pour qu'on puisse le faire mais c'est très rare." (Inspecteur du travail)
\end{abstract}

L'analyse des avis rendus fait parfois ressortir le caractère quelque peu formel de la décision du comité d'entreprise. On ne peut, en effet, que s'étonner, à la lecture de certains dossiers de demandes de licenciements pour motif économique, de constater que la réunion du comité d'entreprise au cours de laquelle l'avis doit être rendu constitue aussi le moment où la direction expose ses projets de restructuration. Cette concomitance est d'autant plus étonnante que le comité d'entreprise devait légalement avoir été déjà consulté sur des projets de ce type ${ }^{1}$. Dans d'autres dossiers, on ne comprend pas pourquoi il n'est fait aucune référence aux consultations et avis rendus antérieurement au projet de restructuration. On peut alors légitimement s'interroger sur la capacité d'appréhension, par le comité d'entreprise, de la situation globale de l'entreprise, tant au plan économique que social. Ce constat de "déconnexion" entre le volet économique et social peut donner à penser que le rôle du comité d'entreprise est alors quelque peu "formel".

Cette faible prise des IRP sur les données économiques, notamment lors de successions de plans de sauvegarde de l'emploi, de fusions, de cessions... se révèle lors d'entretiens réalisés avec des secrétaires de comité d'entreprise. Les représentants des salariés sont démunis;

"On était assez perdus. [...] On avait tellement entendu de choses avant. [...] On ne savait plus ce que l'on devait croire" (Secrétaire de comité d'entreprise, PME)

Les représentants du comité d'entreprise connaissent des difficultés pour appréhender une réalité économique et juridique extrêmement complexe, qui échappe aux béotiens.

"En France, dans l'opinion publique commune [...] on finit presque par admettre que dès lors qu'on dit que c'est économique, il n'y a même pas à contrôler que ce soit fondé"." (Inspecteur du travail)

Le recours à un avocat permet alors parfois de combler ces carences.

Cette faible prise sur les contexte économique et social s'explique également, pour partie, par la faible culture économique et juridique des membres du comité d'entreprise qui demeurent insuffisamment formés malgré le droit à congé dont ils disposent ${ }^{2}$.

Au bout du compte, la relativité de l'avis de comité d'entreprise s'explique aussi par l'absence d'effet contraignant.

\footnotetext{
${ }^{1}$ Art. L. 432-1, $2^{\mathrm{e}}$ al. du Code du travail.

${ }^{2}$ Suivant l'article L. 451-1, $4^{\mathrm{e}}$ al. du Code du travail, les salariés désireux de participer à des stages ou sessions de formation économique, sociale ou syndicale ont droit à des congés rémunérés dont la durée totale ne peut excéder, par an et par salarié, "douze jours ou dix-huit jours pour les animateurs des stages et sessions et pour les salariés appelés à exercer des responsabilités syndicales. La durée de chaque congé ne peut être inférieure à deux jours".
} 
"[...] C'est vrai que souvent les entraves aux IRP c'est souvent du formalisme, d'autant plus que le comité d'entreprise n'a pas un pouvoir de blocage il n'a qu'un pouvoir de consultation, donc, allez expliquer [aux magistrats] que c'est porter gravement atteinte à l'ordre public social que de ne pas avoir consulté le comité d'entreprise en amont sur telle décision, alors que le comité d'entreprise ne peut que dire : on n'est pas d'accord mais l'employeur n'en a rien à foutre et il peut quand même continuer à avancer, c'est un petit exercice intellectuel." (Inspecteur du travail)

\section{B) L'organisation syndicale}

L'intervention, au cours du conflit, de l'organisation syndicale contribue à figer le conflit (1). Elle apparât, dès lors que le litige est né, comme une ressource mobilisée par le salarié protégé (2).

\section{1) La présence syndicale cristallise le conflit}

L'analyse de terrain montre que la présence syndicale cristallise le conflit : elle produit des effets différents selon le moment auquel elle intervient. Incontestablement, l'affiliation à un syndicat par un salarié protégé est mal perçue par l'employeur, comme si le salarié faisait entrer l'ennemi dans l'entreprise. Cela peut être vécu comme une déclaration de guerre dont la réponse est le licenciement du salarié.

\footnotetext{
"Je vais juste vous donner un petit détail sur pourquoi on s'est présenté : c'est Y qui, le premier, est allé chercher son mandat pour créer la cellule et se présenter aux élections. 15 jours après il avait une convocation pour licenciement." (Salarié protégé, délégué syndical, cadre, 42 ans, Bac, PME, demande de licenciement pour faute)
}

Cette analyse est corroborée par des responsables syndicaux locaux : selon le secrétaire général d'une Union départementale CGT, P. Varelat, on a de plus en plus de recours, dans les entreprises moyennes, aux demandes d'autorisation lors des périodes de revendication; le licenciement apparaît alors lié à l'implantation syndicale.

La vision d'un syndicat "belliciste" trouve un écho chez certains salariés protégés nonsyndiqués qui, expliquant leur non-affiliation, font état de leur volonté de rester "neutre", des risques d'être "bloqué" par un syndicat. Pour un autre salarié protégé, le syndicat est synonyme d'agressivité.

"Je pense qu'on se syndique quand on veut être beaucoup plus agressif, je pense qu'en s'expliquant bien, on n'en a pas besoin." (Salariée, membre du comité d'entreprise, déléguée du personnel, non-syndiquée, cadre, 54 ans, BEPC, PME, demande de licenciement économique)

Le recours au syndicat intervient comme une arme défensive, ainsi que l'explique une salariée protégée secrétaire de comité d'entreprise.

"Pour se défendre; [ avant la succession de plans de licenciements], je n'avais pas de raisons d'être syndicaliste [...]. Je suis [à la CFDT] depuis 6 ans maintenant suite aux menaces que nous avons eues parce qu'il n'y a pas que moi, et avec plusieurs personnes ; pour se défendre on a crée un syndicat." (Secrétaire du comité d'entreprise, déléguée syndical, 58 ans, PME)

De ce fait, le mandat sert aussi parfois les intérêts immédiats de celui qui l'occupe, qu'il s'agisse de petites ou de grosses entreprises. Le salarié peut chercher à se protéger, ce qui est 
le cas dans cette entreprise de type paternaliste, divisée en petits établissements répartis sur la France entière.

"[Le PDG] nous a demandé pourquoi on s'était syndiqué, on lui a répondu que c'est parce qu'on voulait passer au $1^{\text {er }}$ tour et avoir une protection parce qu'on avait peur." (Salarié protégé, délégué syndical, cadre, 42 ans, Bac, PME, demande de licenciement pour faute)

Un autre salarié protégé de la même entreprise confirme cette analyse.

" Quand on s'est syndiqué, c'était uniquement pour être réglo, on n'en avait rien à foutre des syndicats, on ne savait même pas comment ça marchait vraiment, on était même assez sceptique par rapport à tout ça. On avait une image des syndicats qui n'était pas très bonne, pas très valorisante, mais on se disait que pour être élu, le meilleur moyen, c'était d'être syndiqué. On était aussi conscient que ça pouvait nous apporter aussi des aides en termes de formation, en termes de soutien, de beaucoup de choses." (Salarié protégé, membre du comité d'entreprise, délégué du personnel, syndiqué, 37 ans, cadre, Bac+2, PME, demande de licenciement pour faute)

Le salarié protégé peut également se servir de son statut de représentant du personnel afin de bénéficier des mesures qu'il a lui-même contribué à mettre en place par la négociation : c'est le cas dans un grand groupe ayant mis en place deux plans sociaux particulièrement attractifs. Si les salariés se servaient de leur mandat pour rester, aujourd'hui ils s'en servent pour partir. Il faut sans doute y voir, pour la responsable des relations sociales, le résultat d'une culture spécifique.

"Une culture où tout se négocie amenée par les plans sociaux [...] où les délégués [...] se servent
les premiers ". (Pourquoi avez-vous accepté les salariés protégés dans le plan, parce que ça fait
quand même des procédures en plus ?) Je pense que si on avait refusé, on se serait fait lyncher vu
le niveau de demandes et de pression qu'il y avait. Moi ça me posait vraiment beaucoup de
questions, la réunion de DP tournait à de la consultation individuelle, les DP étaient là et posaient
des questions pour eux. Il y en avait un en particulier, qui est parti dans le cadre de ce plan et dont
je me souviendrai pendant très longtemps, ils étaient dans une logique qui me rappelle la revue "Le
Particulier", de comment je peux gagner encore plus, comment je peux faire encore plus. Donc ils
grattaient, ils étaient tous avec leur logiciel Excel dans tous les sens, ils faisaient des simulations,
et ils revenaient avec des questions très pointues, c'était impressionnant parce que du coup, on
retrouvait toute la force de [l'entreprise]. Ils faisaient des groupes de travail (C'était l'autogestion
?) Oui, mais dans l'esprit de [l'entreprise], c'est normal, dans la culture qualité de [l'entreprise],
quand quelque chose ne va pas, on fait un groupe de travail, alors en plus, comme c'était des
anciens, ils étaient piqués à cette culture-là. Donc ils ont fait exactement comme ils auraient fait
avec un problème business de base, dans lesquels ils faisaient des 'dé-briefring',, des négo, donc
ils faisaient des allers et retours, ce qui faisait un partenaire de plus." (DRH, grande entreprise)

\section{2) Le syndicat : une ressource dans le litige}

L'analyse des dossiers administratifs de demandes de licenciements de salariés protégés montre une absence de l'organisation syndicale au cours de la procédure. Bien que le droit l'autorise à venir accompagné d'un représentant syndical, le salarié protégé se rend généralement seul à l'enquête contradictoire.

"Cela fait partie du constat que je disais sur la faiblesse des organisations syndicales, les syndicats défendent peu leurs délégués. Tous les ans, la CGT nous sort des tirades infernales sur le fait que l'inspection du travail a encore autorisé je ne sais plus quel pourcentage ! Globalement, c'est vrai, cette statistique en tant que telle ne veut pas forcément dire énormément de choses, parce que quand on voit comment les délégués sont défendus par leurs syndicats, c'est éloquent." (Inspecteur du travail) 
On constate que c'est le salarié protégé, dès lors qu'il est en capacité de le faire, qui mobilise l'organisation syndicale qui sert alors de ressource : elle informe le salarié protégé sur le droit et sur ses droits, et apporte, le cas échéant, un soutien financier :

"(Et l'organisation syndicale vous a aidé ?) Oui, là-dessus, ils nous ont conseillé, ils sont venus au tribunal avec nous, ils nous ont envoyé l'avocate qui était très bien." (Secrétaire du comité d'entreprise, déléguée syndical, 58 ans, PME)

L'organisation syndicale offre alors un soutien logistique plus que politique. Elle intervient lors du litige, c'est-à-dire lorsqu'un recours administratif ou contentieux est enclenché. Il se traduit généralement, au niveau des pratiques syndicales, par une protection plus importante accordée au représentant syndiqué (assistance juridique par exemple). D'autre part, les actions syndicales extérieures à l'entreprise sont aussi destinées à montrer que le syndicat défend ses adhérents. Certains dossiers occupent une plus grande place; l'investissement de l'organisation syndicale varie en fonction de la taille de l'entreprise, des enjeux politiques et de l'implantation syndicale.

Le soutien apporté par le syndicat au salarié protégé dans le cadre de l'entreprise demeure faible. Ce constat s'explique, pour partie, par le fait qu'un tel soutien politique ne dépend pas que du syndicat mais aussi de la capacité du salarié protégé à mobiliser les autres salariés, comme l'explique un salarié protégé.

\begin{abstract}
"La CGC m'a soutenu, ils voulaient aller au conflit, ils voulaient que je syndique des gens, il fallait trois syndiqués par mois. Je n'avais pas l'habitude de travailler comme ça moi, je ne sais pas gérer comme ça, je leur ai dit. Ils m'ont répondu : on ne peut rien pour vous dans ces conditions, vous ne faites pas masse. Je leur ai dit : "je comprends, mais je ne peux pas faire signer de force les gens ou les enrôler sans qu'ils le sachent, ce n'est pas possible, je vais leur payer leurs cotisations à la limite. On ne fait pas comme ça ![...] C'est vrai que je n'ai pas eu le rôle véritable de délégué syndical, je l'ai fait, comme je vous l'ai dit tout à l'heure, j'ai été délégué syndical parce que personne au comité d'entreprise n'a voulu prendre la responsabilité et je les comprends. J'étais le moins facile à faire plier" (Salarié protégé, délégué syndical, membre du comité d'entreprise, 55 ans, cadre, BEPC, PME, demande de licenciement pour inaptitude)
\end{abstract}

Or le statut juridique joue alors pleinement son rôle dès lors que le salarié protégé est soutenu par un collectif de travail. Ce collectif se délite cependant d'autant plus que la présence syndicale est faible.

\footnotetext{
"Il y a toujours la peur du patron quelque part, les gens ont peur, ils veulent bien faire les choses, mais il ne faut pas que ça se sache de trop. (Vous ne pouvez donc pas vous appuyer sur le collectif du travail pour défendre votre propre situation ?) Non. Nous, c'était essentiellement des bureaux à N., c'est difficile parce que les gens n'osent pas et ne se sentent pas vraiment concernés, ils ne veulent pas faire de vagues, ils ont toujours l'espoir qu'en fermant leurs gueules, le patron en tiendra compte. Quelque part, on est encore au $19^{\text {ème }}$ siècle." (Membre du CE, syndiqué, PME)
}

L'organisation syndicale peut apparaître déterminante dans l'issue de la procédure lorsque la présence syndicale est forte au sein de l'entreprise et qu'elle représente un véritable contrepouvoir ; c'est ce que dit, de manière indirecte, un inspecteur du travail .

"Les seules chances, quand je reprends les nombreux dossiers que j'ai vu passer entre mes mains sur des licenciements, les seules chances qu'un refus puisse avoir réellement des chances de se traduire complètement par la construction d'un contre-pouvoir syndical dans l'entreprise, c'est quand il y a déjà une présence syndicale forte, qui fait que le salarié dont j'ai refusé le licenciement, est réintégré dans une communauté de salariés où il sera soutenu, où ils peuvent se 
serrer les coudes entre eux, et où ils peuvent éviter que la direction remette le paquet pour remonter une nouvelle fois un dossier de licenciement." (Inspecteur du travail)

\section{Le soutien politique ne peut intervenir sur le conflit sans ancrage syndical fort :}

"Ils [la CGC] voyaient en moi une nouvelle entreprise sur XXX [un village] avec plein de syndiqués, ça moi, je n'ai pas su faire. Quand j'en ai parlé à mes collègues, ils m'ont dit : mais tu es fou, tu veux qu'on soit viré tout de suite ? C'est ce genre de paroles, vous ne pouvez pas vous battre contre ça, les gens qui ne veulent pas ne veulent pas. Le pire c'est qu'ils sont conscients de la situation sans pouvoir y amener quelque chose pour la modifier, je crois que c'est la plus difficile des situations, ça. "(Salarié protégé, délégué syndical, membre du comité d'entreprise, 55 ans, cadre, BEPC, PME, demande de licenciement pour inaptitude) 


\title{
Conclusions intermédiaires
}

Les tiers institutionnels influencent donc inégalement l'issue de la procédure. L'inspecteur du travail y occupe une place fondamentale : c'est lui qui rend la décision. Mais il n'est pas pour autant un juge, tant au plan de l'analyse juridique que des pratiques. La nature de son intervention est fondamentalement différente. Il n'est pas chargé de "dire le droit", comme l'est un juge, mais de rendre une décision au nom du droit. Ce faisant, il tient compte des rapports sociaux. Son rôle est primordial dès lors qu'il permet de re-situer un conflit dans son histoire sociale et collective afin de lui donner un sens. Il n'est pas rare qu'une demande individuelle de licenciement s'inscrive dans une histoire collective que seule la connaissance du contexte socio-économique permet de saisir. Le rôle de l'inspecteur du travail consiste, par conséquent, à apprendre à composer avec le contexte. C'est ce qu'exprime à sa manière un inspecteur du travail, s'agissant des recours aux transactions qui traduisent, sous l'habillage du licenciement, l'existence d'un départ négocié ${ }^{1}$;

\begin{abstract}
"Et là, effectivement, je me mets à [la] place [du salarié protégé] en me disant, moi j’ai la sécurité de l'emploi, c'est très facile de dire à quelqu'un, "vous resterez dans l'entreprise, et bannière en avant, et droit du travail, etc..." D'ailleurs c'est une question que j'aimerais bien poser un jour à un conseiller d'état, qui lui aussi a la sécurité de l'emploi. Mais je comprends en même temps très bien la position du Conseil d'Etat, et je trouve qu'après tout, c'est intéressant qu'il ait cette position, qui est une position assez solennelle, d'affirmation, c'est la procédure exorbitante du droit commun par rapport à un droit constitutionnel, le droit de la représentation du personnel. C'est quelque chose, une valeur républicaine qu'il faut affirmer fortement. Après nous, on fait avec les réalités, avec le terrain... Mais en même temps, il est sûr qu'on prend des décisions illégales." (Inspecteur du travail)
\end{abstract}

Le droit invite d'ailleurs l'inspecteur du travail à replacer une demande individuelle dans son contexte : si l'employeur doit rechercher à reclasser le salarié déclaré inapte (art. L. 122-325), il incombe à l'inspecteur du travail de vérifier "la portée des mesures de reclassement qui ont été proposées compte tenu des conclusions du médecin du travail et au regard des circonstances de l'affaire si le licenciement n'est pas en rapport avec le mandat de l'intéressé" ${ }^{2}$. Une telle analyse implique une connaissance du terrain. "[...] Dans le cas où la demande de licenciement est motivée par l'inaptitude physique du salarié, il appartient à l'inspecteur du travail de rechercher, sous le contrôle du juge de l'excès de pouvoir, si cette inaptitude est telle qu'elle justifie le licenciement, compte tenu des caractéristiques de l'emploi exercé à la date à laquelle elle est constatée, des règles applicables au contrat de travail du salarié, des exigences propres à l'exécution normale du mandat dont il est investi et de la possibilité d'assurer son reclassement dans l'entreprise" ${ }^{\prime 3}$.

Quant au comité d'entreprise et à l'organisation syndicale, leur place apparaît tout à fait limitée dans l'issue de la procédure. Il pourrait en aller autrement dès lors que les syndicats décident de concrétiser leur volonté de "resyndiquer" les comités d'entreprise ${ }^{4}$, ce que semblent montrer les dernières élections professionnelles avec un recul des listes nonsyndiquées.

\footnotetext{
${ }^{1}$ Illicite s'agissant des salariés protégés ; supra, Ière partie.

${ }^{2}$ CE 22 mai $2002 \mathrm{n}^{\circ} 221600$, Sté civile d'Arbonne, RJS 8-9/02, n 1000.

${ }^{3} \mathrm{CE} \mathrm{n}^{\circ} 171476,15$ juin 1998 Onet Propreté.

${ }^{4}$ Lire L. Abrassart, "Les syndicats à la reconquête des CE", Le Monde initiatives novembre 2003.
} 


\section{III $^{\mathrm{E}}$ PARTIE - LE LICENCIEMENT ENTRE ARRANGEMENT ET SORTIE DE CRISE}

Une fois la décision de l'inspecteur du travail rendue, deux perspectives s'ouvrent aux parties : la persistance (I) ou la fin du conflit (II).

\section{I- Le licenciement peut être vécu comme inéluctable ou avantageux}

Signalons tout d'abord que le volume des dossiers de demande de licenciements constitués par les inspecteurs du travail est très fluctuant. Certains dossiers sont uniquement constitués de la demande de licenciement de l'entreprise et du refus (rare dans ce cas) ou de l'accord de l'inspecteur du travail. Cette épaisseur ne dit rien de la nature des relations entre salariés et l'entreprise. Par contre, elle indique clairement que le motif de licenciement apparaît indubitable pour les salariés ou, ce qui revient pratiquement au même, que ceux-ci se trouvent dans une telle position de faiblesse qu'ils sont dans l'incapacité d'argumenter.

En effet, certains licenciements ne donnent pas lieu à dispute car les raisons invoquées sont acceptées de part et d'autre. Le licenciement pour motif économique, notamment lorsqu'il est collectif, en est une illustration. Si, dans certains cas, le licenciement, n'est pas accepté de "gaieté de cœur", il apparaît aussi comme une réalité dont toutes les parties sont conscientes. La dégradation, depuis le milieu des années soixante-dix, du marché de l'emploi ainsi que les fermetures d'entreprise ont rendu cette réalité difficilement contournable.

De ce fait, la protection accordée aux représentants du personnel doit être relativisée et mesurée à l'aune de l'évolution du marché du travail. En effet, dans une période de plein emploi, le licenciement apparaît comme une mesure exceptionnelle. Il peut alors avoir pour objectif de se débarrasser d'un gêneur ou se révéler peut être une mesure antisyndicale. À l'inverse, la banalisation du chômage donne un tout autre sens à la demande de licenciement d'un représentant du personnel : si tout le monde peut être licencié, pourquoi serait-il protégé ? L'acceptation du licenciement économique va alors presque de soi.

\footnotetext{
"C'était un motif économique, elle était d'accord pour reconnaître que l'entreprise allait très mal et les chiffres financiers, c'est elle-même qui me les avait fournis d'ailleurs. [...] Mais en tout cas, elle ne contestait absolument pas la réalité du motif économique, et elle m'avait adressé un courrier où ces chiffres figuraient [...] Il était donc difficile de nier la réalité des difficultés économiques de l'entreprise. [...] On voyait bien que l'activité de l'entreprise était un peu en péril. Là-dessus, le motif, par rapport aux critères de jurisprudence, était difficilement niable. Sur la réalité de la suppression de son poste, il n'y avait pas d'autres postes équivalents à celui qu'elle occupait dans l'entreprise.» (Inspecteur du travail)
}

Dans ce cas précis, le licenciement apparaît comme une contrainte exogène que Reynaud (1989) oppose aux contraintes endogènes (qui résultent des volontés des parties qui se rencontrent dans l'accord) et qui s'impose aux parties indépendamment de leur volonté. Le licenciement devient alors, sous réserve que les salariés portent la même appréciation sur les difficultés économiques de l'entreprise, affaire de «pas de chance» ou, pour le dire autrement, comme un effet mécanique du fonctionnement du marché.

Ce constat se vérifie d'autant plus dans les procédures de règlement judiciaire où le retrait des dirigeants de la gestion de l'entreprise, associé à l'intervention d'un administrateur, marque 
symboliquement ce glissement vers une gestion de «l'urgence économique » sur laquelle les dirigeants antérieurs n'ont que peu de prise. Corrélativement, la présentation que font les administrateurs de leur rôle apparaît circonscrite au champ de la technique juridique ou encore de l'intervention consensuelle.

"Il faut quand même savoir que le liquidateur n'est pas forcément l'ennemi du salarié, bien au contraire, il hérite d'une situation[...] donc il n'y a pas de raison pour que ça se passe mal." (Administrateur judiciaire, PME)

En outre, la relative objectivation des critères du licenciement conduit parfois le salarié licencié à appréhender son licenciement sous le seul angle technique, c'est-à-dire comme une procédure qui n'est pas dirigée contre lui ou dont il pourrait porter la responsabilité. La sortie de l'entreprise paraît alors, à terme, inéluctable. De la même manière, la dégradation des relations sociales contribue à rendre moins douloureux le départ de l'entreprise.

Parce qu'on est licencié, on bénéficie du chômage donc c'est plus facile de partir, si on démissionne, on n'a rien. Moi dans mon cas, je n'en pouvais plus, l'ambiance au niveau du service n'était pas terrible.» (Salariée protégée, déléguée du personnel, membre du CHSCT, nonsyndiquée, 33 ans, CAP, agent de maîtrise, PME, demande de licenciement économique) ${ }^{1}$

Le manque de confiance dans l'avenir de l'entreprise associé à une certaine dégradation des relations sociales devient alors un puissant motif de départ. Certains licenciements économiques ne sont, d'autre part, pas perçus par le salarié protégé comme des licenciements mais plutôt comme des mesures d'âge, une opportunité de départ avant le terme. Quant aux licenciements collectifs pour motif économique, ils peuvent intégrer un plan de volontariat avec des mesures incitatives qui rendent la sortie parfois préférable au maintien dans l'emploi. Si le salarié est en fin d'activité ou qu'il a un emploi assuré, le licenciement d'une entreprise déclinante peut apparaître comme une occasion à ne pas rater.

Il n'y a dès lors rien d'étonnant à ce que certains licenciements collectifs se fassent sur la base du volontariat. Par un renversement de perspective, la sortie de l'entreprise apparaît comme un sort enviable. Dans un tel contexte, vécu comme inéluctable ou encore comme une (relative) «aubaine », le licenciement économique paraît s'imposer, tant au salarié protégé qu'à l'inspecteur du travail qui subit souvent de très fortes pressions des salariés eux-mêmes pour autoriser le licenciement et rendre rapidement une décision.

\begin{abstract}
"À la demande de mon licenciement, il a fallu que je m'y reprenne 4 à 5 fois pour gagner un mois avec [l'inspecteur du travail. Il ne voulait] absolument pas. [...] Il savait que personnellement, il a reçu 2 ou 3 écrits de ma part, comme quoi c'était volontaire, comme quoi c'était un plan, je lui ai même dit à un certain moment. [...] Je lui ai dit, mais vous vous rendez compte, vous allez imposer à la Sécurité Sociale de payer. Parce que moi, de toute façon, avant le plan, j'avais prévu de me faire opérer des varices. [...] Tout mon mois de Février a été en arrêt de travail, je suis revenu 4 ou 5 jours, et là, je lui ai renvoyé un autre mot où je lui ai dit que le mois de Mars risque d'être pareil. [...] Je dois vous dire honnêtement que j'ai fait le forcing. [...] À la fin, il devait en avoir marre, il devait se dire : "celui-là, je vais encore recevoir 2 ou 3 lettres de sa part, etc..." (Salarié protégé, secrétaire du CHSCT, non-syndiqué, 55 ans, cadre, BTS, CE-CHSCT, grande entreprise, licenciement économique)
\end{abstract}

Il n'est donc pas surprenant, dans un tel contexte, que le pourcentage d'acceptation des demandes de licenciements pour motif économique par les inspecteurs du travail soit élevé

\footnotetext{
${ }^{1}$ Toutefois, rien ne dit que cet optimisme soit justifié. Ainsi la situation de cette salariée deux ans après est loin de correspondre à ses prévisions : " C'est la merde, je viens de faire un dossier de surendettement, voilà où j'en suis actuellement".
} 
(91\%, alors qu'il n'est que $63 \%$ s'agissant du motif disciplinaire et $77 \%$ pour le motif professionnel $^{1}$ ).

D'autre part, le motif économique est deux fois plus souvent avancé dans les demandes de licenciement de salariés protégés que pour l'ensemble des licenciés qui s'inscrivent à l'ANPE $^{2}$. On peut donc tout à fait faire l'hypothèse que certaines entreprises saisissent cette occasion pour tenter de se débarrasser de certains salariés protégés.

\section{II- La persistance du conflit}

La persistance du conflit peut se traduire, au plan de l'analyse juridique, par la mise en œuvre d'un recours administratif ou contentieux (A) qui peut aussi être utilisé dans la recherche de l'écrasement de l'adversaire (B).

\section{A) Le recours administratif ou contentieux}

Deux voies, alternatives ou cumulatives, s'ouvrent aux parties à la procédure (le salarié ou l'employeur) dès lors qu'elles contestent la décision rendue par l'inspecteur du travail et en demandent l'annulation ou la réparation pécuniaire : le recours administratif ou le recours contentieux.

Le recours administratif, c'est-à-dire porté devant l'administration elle-même, peut s'adresser à l'autorité qui a émis l'acte (recours gracieux) ou à l'autorité supérieure (recours hiérarchique porté devant le Ministre chargé du travail). Il n'a pas d'effet suspensif: dès lors, le licenciement autorisé par l'inspecteur du Travail produit tous ses effets : le salarié qui a été licencié de son travail n'est par conséquent plus tenu à l'obligation de loyauté qui pesait sur lui pendant la durée du contrat ${ }^{3}$. Les services du Ministère du travail en charge $d u$ dossier litigieux peuvent désigner un échelon intermédiaire (région ou département) qui se verra confier la charge de réaliser une contre-enquête et de rédiger une note de synthèse. La circulaire DRT $\mathrm{n}^{\circ} 3 \mathrm{du} 1^{\mathrm{er}}$ mars $2000^{4}$ prévoit qu'ils doivent recevoir les pièces qui ont été communiquées par les parties à l'inspecteur du travail. Le Ministre n'est pour autant pas tenu, selon la jurisprudence du Conseil d'Etat, de faire procéder à une enquête contradictoire ${ }^{5}$.

Les parties peuvent également contester soit directement la décision de l'inspecteur du travail, soit la décision rendue dans l'affaire par le Ministre. Le recours devient alors juridictionnel car porté devant les juridictions administratives (Tribunal administratif, Cour administrative d'appel, Conseil d'Etat). En vertu du principe de séparation des pouvoirs ${ }^{6}$, il appartient aux seules juridictions administratives de se prononcer sur la légalité d'une décision administrative. L'annulation, par le Conseil d'Etat, de la décision de refus d'autorisation du

\footnotetext{
${ }^{1}$ Merlier R. (2002), "Les licenciements de représentants du personnel en 2000", Premières informations, novembre 2002, $\mathrm{n}^{\circ} 48.2$

${ }^{2}$ En 2003, deux tiers des demandes de licenciement de salariés protégés se fondent sur un motif économique tandis que seulement un tiers des salariés licenciés s'inscrivant à l'ANPE déclarent avoir été licenciés pour motif économique.

${ }^{3} 12$ mai 1998 - No 247 , Sociale

${ }^{4}$ Circulaire DRT n 3 du $1^{\text {er }}$ mars 2000, Ministère de l'emploi et de la solidarité, Dr. Ouvrier oct. 2000, p. 442 à 451

${ }^{5} \mathrm{CE} 22$ mai 2002, $\mathrm{n}^{\circ} 214637$, Zaugg, RJS 8-9/02 V, nº 999.

${ }^{6}$ Posé en principe par les articles 10 et 13 de la loi des 16 et 24 août 1790 et le décret du 16 fructidor An III et consacré par le Conseil constitutionnel (Déc. $\mathrm{n}^{\circ}$ 86-224 DC du 23 janvier 1987, considérant $\mathrm{n}^{\circ} 15$, JO de 25 janvier, p. 924); cf. J.-Y. Kerbourc'h, Le statut protecteur des représentants du personnel, LITEC, 2003, 343 p., p. 263.
} 
licenciement ne peut valoir autorisation de licenciement ${ }^{1}$. Il appartient alors, le cas échéant, à l'employeur, de formuler une nouvelle demande auprès de l'inspection du travail.

Les tribunaux judiciaires (Conseil de prud'hommes, Cour d'appel, Cour de cassation) doivent surseoir à statuer dans l'attente de la décision de la juridiction administrative ${ }^{2}$, même si, comme le relève J-Y Kerbourc'h, "il n'est pas rare que le juge judiciaire méconnaisse l'autorité de la chose décidée par le juge administratif, que ce dernier confirme ou annule l'autorisation de licencier" ${ }^{\prime 3}$. Il est aussi tenu, sur le fond, par la décision de la juridiction administrative $e^{4}$ : un tribunal administratif ayant retenu que l'autorisation de licencier un salarié reposait sur des faits matériellement inexacts, la cour d'appel aurait dû en déduire nécessairement l'inexistence d'une cause réelle et sérieuse de licenciement ${ }^{5}$. Par conséquent, le juge judiciaire ne peut apprécier le caractère réel et sérieux du licenciement; il doit, le cas échéant, surseoir à statuer en renvoyant l'appréciation de légalité à la juridiction administrative ${ }^{6}$. Il demeure cependant compétent pour statuer sur les licenciements prononcés en dépit ou sans autorisation administrative ou encore sur les demandes de réintégration ou d'indemnisation.

S'il ne peut se prononcer sur l'existence d'une faute commise par le salarié, il peut la qualifier (grave ou lourde) mais est tenu de n'examiner que les fautes retenues par l'autorité administrative (encadré)

\begin{tabular}{l} 
Cass. Soc. $\mathbf{1 0}$ juillet $\mathbf{2 0 0 1}$ - $\mathbf{N}^{\circ} \mathbf{2 6 0}$ \\
REPRÉSENTATION DES SALARIÉS. - Règles communes. - Contrat de travail. - Licenciement. - \\
Mesures spéciales. - Autorisation administrative. - Portée. \\
Cassation. \\
$\mathrm{N}^{\circ} 98-42.808$ \\
$\mathrm{M}$. WAQUET, conseiller doyen faisant fonctions de président \\
Sur le moyen unique: \\
Vu l'article L. 425-1 du Code du travail; \\
Attendu que M. Clémot, engagé en 1976 par la société Martin-Rondeau en qualité d'attaché commercial, élu \\
délégué du personnel à partir de 1990, a été licencié pour faute grave par lettre du 24 juin 1994 après \\
autorisation donnée par l'inspecteur du travail et fondée d'une part, sur l'existence d'activités personnelles \\
pendant le temps de travail et d'autre part, sur l'établissement de faux rapports de visite; que sur recours \\
hiérarchique, le ministre du travail n'a retenu que le premier grief pour confirmer la décision de l'inspecteur \\
du travail; \\
Attendu que pour dire que le licenciement de M. Clémot reposait sur une faute grave et rejeter ses \\
demandes d'indemnités, la cour d'appel retient que l'existence de faux rapports de visite établie par les \\
pièces versées aux débats suffit pour dire que la société Martin Rondeau apporte la preuve de faits \\
imputables à Jacques Clémot constituant une violation des obligations découlant du contrat de travail d'une \\
importance telle qu'elle rendait impossible le maintien de celui-ci dans l'entreprise, même pendant la durée \\
limitée du préavis; \\
Attendu, cependant, que seul le ou les motifs retenus par l'autorité administrative pour autoriser le \\
licenciement disciplinaire d'un représentant du personnel peuvent justifier ce licenciement; que le juge \\
judiciaire, compétent pour apprécier si le salarié protégé a commis une faute grave ou une faute lourde, ne \\
peut examiner que les fautes retenues par l'autorité administrative; \\
Qu'en statuant comme elle l'a fait, alors que pour confirmer l'autorisation de licenciement, le ministre du \\
travail n'a retenu à l'encontre de M. Clémot que le grief tiré d'activités personnelles pendant le temps de \\
travail, la cour d'appel a violé le texte susvisé; \\
\hline
\end{tabular}

\footnotetext{
${ }^{1}$ Cass. Soc. 26 juillet $1984-\mathrm{N}^{\circ} 336$.

${ }^{2}$ Cass. soc. 3 juillet 1985 - $\mathrm{N}^{\circ} 391$ : "C'est à bon droit qu'une cour d'appel a sursis à statuer sur la demande de réintégration d'un salarié protégé jusqu'à ce que la juridiction administrative compétente se soit définitivement prononcée sur la question préjudicielle de la légalité de la décision du ministre qui a annulé sa précédente décision".

${ }^{3} \mathrm{~J}$-Y Kerbourc'h, Le statut protecteur des représentants du personnel, Litec, 2003, 343 p., p. 256.

${ }^{4}$ Cass. Soc. $1^{\text {er }}$ oct. 2003, Abdel Malek Boucherit et autres c/ Sté Entrepose Montalev, Dr. Soc. 2003, p. 1136 à 1138 , note J. Savatier.

${ }^{5}$ Cass. Soc. 12 mars 1987 - No 149.

${ }^{6}$ Cass. Soc. 25 novembre $1997-\mathrm{N}^{\circ} 399$.
} 


\section{PAR CES MOTIFS:}

CASSE ET ANNULE, dans toutes ses dispositions, l'arrêt rendu le 17 mars 1998, entre les parties, par la cour d'appel d'Angers; remet, en conséquence, la cause et les parties dans l'état où elles se trouvaient avant ledit arrêt et, pour être fait droit, les renvoie devant la cour d'appel de Rennes;

M. Clémot contre société Martin Rondeau.

Président: M. Waquet, conseiller doyen faisant fonction. - Rapporteur: M. Lanquetin. - Premier avocat général: M. Benmakhlouf. - Avocats: la SCP Célice, Blancpain et Soltner, la SCP Piwnica et Molinié.

D'autre part, si l'examen de la légalité de la décision de l'autorité administrative autorisant ou refusant la rupture d'un contrat de travail incombe aux juridictions administratives, les juridictions judiciaires demeurent compétentes pour octroyer des dommages-intérêts (encadré) :

\begin{tabular}{|l|}
\hline Cass. Soc. 14 mai 1987 - $\mathbf{N}^{\circ} \mathbf{3 3 0}$ \\
"Doit être cassé l'arrêt ayant déclaré la juridiction prud'homale incompétente pour statuer sur les demandes \\
en paiement des dommages-intérêts pour licenciement sans cause réelle et sérieuse formée par un membre \\
du comité d'entreprise aux motifs que cette juridiction ne pouvait, sans enfreindre le principe de la \\
séparation des pouvoirs, connaître d'une instance dont l'objet remettrait en cause la légalité de la décision \\
administrative en vertu de laquelle le licenciement était intervenu, alors que si la cour d'appel a exactement \\
relevé que la décision de l'inspecteur du travail ne pouvait être critiquée par le salarié que par la voie du \\
recours hiérarchique ou par un recours devant la juridiction administrative intervenant comme juge de \\
l'excès de pouvoirs, elle n'en demeurait pas moins compétente, à l'exclusion des juridictions administratives \\
et après solution le cas échéant par elles des questions préjudicielles pour statuer ensuite sur la demande de \\
l'intéressé en dommages-intérêts pour licenciement sans cause réelle et sérieuse".
\end{tabular}

Suite à une annulation de l'autorisation administrative de licenciement, le salarié peut

1) demander sa réintégration au même poste ${ }^{1}$ et une indemnité compensant la perte de salaire

2) saisir le juge judiciaire aux fins d'obtenir réparation et demander le versement de dommages et intérêts (montant des avantages directs et indirects qu'il aurait dû percevoir à compter de la rupture jusqu'à la fin de la période de protection)

Article L436-3 du Code du travail
(L. n 82-915, 28 oct. 1982)
L'annulation, sur recours hiérarchique, par le ministre compétent d'une décision de l'inspecteur du travail
autorisant le licenciement d'un salarié mentionné aux articles L. 436-1 et L. 436-2 emporte, pour le salarié
concerné et s'il le demande dans un délai de deux mois à compter de la notification de la décision, droit à
réintégration dans son emploi ou dans un emploi équivalent.
Il en est de même dans le cas où, sauf sursis à exécution ordonné par le Conseil d'État, le juge administratif
a annulé une décision de l'inspecteur du travail ou du ministre compétent autorisant un tel licenciement.
Le salarié concerné est réintégré dans son mandat si l'institution n'a pas été renouvelée. Dans le cas
contraire, il bénéficie pendant une durée de six mois, à compter du jour où il retrouve sa place dans
l'entreprise, de la procédure prévue à l'article L. 436-1.
Lorsque l'annulation de la décision d'autorisation est devenue définitive, le salarié concerné a droit au
paiement d'une indemnité correspondant à la totalité du préjudice subi au cours de la période qui s'est
écoulée entre son licenciement et sa réintégration s'il l'a demandée dans le délai, prévu au premier alinéa, ou
l'expiration de ce délai dans le cas contraire. Ce paiement s'accompagne du versement des cotisations
afférentes à ladite indemnité, qui constitue un complément de salaire.

Parallèlement à ces sanctions civiles, le juge pénal peut être saisi et condamner l'employeur pour délit d'entrave.

\footnotetext{
${ }^{1}$ Par exemple, Cass. Soc. 11 décembre 2001, Abderrahim c/ Sté Mesnil G20, Dr. Ouvrier mars 2002, p. 125 et 126, note Milet
} 


\begin{tabular}{|c|}
\hline $\begin{array}{c}\text { CODE DU TRAVAIL } \\
\text { (Partie Législative) } \\
\text { Chapitre } 3 \text { : Les comités d'entreprise } \\
\text { Article L483-1 }\end{array}$ \\
\hline $\begin{array}{l}\text { (Loi } n^{\circ} \text { 83-1179 du } 29 \text { décembre } 1983 \text { art. } 76 \text { Journal Officiel du } 30 \text { décembre } 1983 \text { date d'entrée en } \\
\text { vigueur 1ER janvier 1984) } \\
\text { (Loi } n^{\circ} \text { 92-1336 du } 16 \text { décembre } 1992 \text { art. } 329 \text { Journal Officiel du } 23 \text { décembre } 1992 \text { en vigueur le ler } \\
\text { mars 1994) } \\
\text { (Loi } n^{\circ} \text { 2001-152 du } 19 \text { février } 2001 \text { art. } 1 \text { I Journal Officiel du } 20 \text { février 2001) } \\
\text { Toute entrave apportée, soit à la constitution d'un comité d'entreprise, d'un comité d'entreprise ou d'un } \\
\text { comité central d'entreprise, soit à la libre désignation de leurs membres, soit à leur fonctionnement régulier, } \\
\text { notamment par la méconnaissance des dispositions des articles L. 433-13, L. } 436-1 \text { à L. 436-3 et des textes } \\
\text { réglementaires pris pour leur application, sera punie d'un emprisonnement d'un an et d'une amende de } \\
3750 \text { euros ou de l'une de ces deux peines seulement. } \\
\text { En cas de récidive, l'emprisonnement pourra être porté à deux ans et l'amende à } 7500 \text { euros. }\end{array}$ \\
\hline $\begin{array}{l}\text { Nota : Ordonnance } 2000-9162000-09-19 \text { art. } 3 \text { : à compter du 1er janvier } 2002 \text {, dans tous les textes } \\
\text { législatifs prévoyant des amendes ou d'autres sanctions pécuniaires ou y faisant référence, les montants } \\
\text { exprimés en francs ( } 25000 \mathrm{~F}, 50000 \mathrm{~F} \text { ) sont respectivement remplacés par des montants exprimés en euros } \\
\text { (3750 euros, } 7500 \text { euros). }\end{array}$ \\
\hline
\end{tabular}

À l'inverse de l'inspecteur du travail qui, ainsi que le soulignait le Procureur général Touffait dans les affaires "Perrier", rend un "arbitrage social" car "le litige à régler est beaucoup plus un conflit social au sein d'une entreprise qu'un problème juridique"1, les juridictions interviennent pour "dire le droit" et, ce faisant, donner sa solution à un litige.

\section{B) La recherche de l'écrasement de l'adversaire}

Mais pour s'engager dans des recours administratifs ou judiciaires il faut détenir les ressources notamment cognitives (voir chapitre II) qui le permettent.

Certaines affaires s'arrêtent bien avant, faute d'adversaires. Le licenciement du salarié protégé peut se dérouler en son absence, dans une négociation entre l'entreprise et l'inspecteur du travail. On peut faire l'hypothèse que les autorisations de licenciement par abandon du salarié sont issues des défaites personnelles de ce dernier qui n'a pas les moyens ni de répondre aux actions de l'entreprise ni d'entretenir le conflit.

"L'employeur m'a dit que depuis le 27 Juillet 2002, le salarié ne s'était pas représenté à son poste de travail, qu'il n'était plus en arrêt maladie, donc il se trouvait finalement dans une situation de non-justification de son absence faite au poste. L'employeur a donc envisagé un licenciement pour inaptitude, et non pas pour faute, bien qu'il y ait eu une non-justification de la "non reprise" de son poste de travail depuis le 27 juillet 02. [...] sachant que le salarié n'est pas venu au moment de l'enquête contradictoire." (Inspecteur du travail)

Pour d'autres, au contraire, le conflit prend une tonalité tellement personnelle, les enjeux sont tellement importants, que la seule façon d'arrêter le conflit semble être d'écraser l'adversaire, ainsi qu'en attestent les deux remarques en miroir du salarié protégé et de son DRH.

"Mais attendez, moi j'essaierai 10 fois (d'obtenir le licenciement du salarié protégé) s'il me donne 10 fois l'occasion d'y aller. Mais je n'irai pas 10 fois pour la même chose parce que j'espère qu'il

\footnotetext{
${ }^{1}$ Cass. Chambre mixte 21 juin 1974, Perrier, Dalloz 1974, p. 593, Concl. Touffait, p. 597.
} 
n'est pas idiot au point de faire 10 fois les mêmes bêtises qu'il a faites. Mais quelqu'un qui me vole dans la caisse, quelqu'un qui harcèle un salarié." (DRH, PME)

"(Le DRH) c'est une ordure, je ne négocierai pas avec lui, c'est une salope et je n'en veux pas." (Salarié protégé, membre du comité d'entreprise, délégué du personnel, syndiqué, 37 ans, cadre, $\mathrm{Bac}+2$, PME, demande de licenciement pour faute)

On se trouve ainsi devant des attitudes "jusqu'au boutistes". La répétition de la même formule à différents moments de l'entretien donne une idée de la rage qui habite le salarié.

\begin{abstract}
"Quand j'ai tort, je l'admets, quand j'ai raison, on ne me fera jamais dire que j'ai tort, je ne me coucherai jamais, je ne suis pas quelqu'un qui sait faire ça. [...] Comme je vous l'ai dit, je ne suis pas quelqu'un de malléable, qui plie facilement. [...] Il n'y a pas de mystère, moi je ne me laisse pas faire. Quand j'ai tort, je m'écrase, je sais faire, je sais reconnaître mes torts, mais quand j'ai raison, tant que j'ai un souffle de vie, je lève la tête, et ils le savaient, je lui ai dit ça, je lui ai dit dans ces termes-là." (Salarié protégé, délégué syndical, membre du comité d'entreprise, 55 ans, cadre, BEPC, PME, demande de licenciement pour inaptitude)
\end{abstract}

En effet, notamment quand le conflit dérape, il peut prendre des formes pathologiques.

"Le conflit a démarré, et comme P. n'a pas été capable de le tenir sur un plan juridique, il l'a laissé dériver sur un cadre de mise en cause personnelle, assez vite à mon avis, eh bien il était de plus en plus mal." (Inspecteur du travail)

"C'était l'horreur, ça c'était un fait, mais c'était toujours des choses pas possibles. Il y a même eu des bruits sur des soi-disant coucheries, je peux en citer parce que des choses comme ça, il y en a eu tout le long pendant 2 ans." (Salarié protégé, délégué syndical, membre du comité d'entreprise, 55 ans, cadre, BEPC, PME, demande de licenciement pour inaptitude)

Dès lors, le conflit ne peut être qu'exacerbé pour les deux parties, comme le reconnaît par exemple ce salarié protégé après avoir clairement affirmé jusqu'où il était prêt à aller.

"J'aurai été radieux, ravi, qu'ils soient condamnés pour harcèlement moral, tout le monde attendait qu'ils soient condamnés pour harcèlement moral, ça aurait été les premiers en France. (La direction a dit) il faut le virer parce qu'il va nous rendre fou." (Salarié protégé, délégué syndical, membre du comité d'entreprise, 55 ans, cadre, BEPC, PME, demande de licenciement pour inaptitude)

\title{
Dès lors, ce sont souvent les interventions de tiers qui permettent de dénouer le conflit
}

"Le médecin du travail a considéré au bout d'un moment qu'il valait mieux que P. quitte l'entreprise pour inaptitude plutôt que de voir sa santé mentale atteinte par un climat qu'il ne supportait plus. [...] Il estimait que c'était de son devoir de protéger P. en quelque sorte, peu importe. C'est vrai que nous, dans les affaires de harcèlement, on cherche à impliquer les médecins du travail puisqu'ils sont quand même bien placés pour intervenir là-dessus, donc on essaie de leur demander de prendre leurs responsabilités." (Inspecteur du travail)

"Quand je l'ai engagé (l'avocat), je leur ai dit : "je ne négocie pas". [...] J'ai discuté avec mon avocat qui m'a dit qu'il n'avait jamais vu un dossier comme ça. Le dossier était quand même conséquent. Il m'a dit : il est à charge et si vous engagez une procédure de harcèlement moral, vous gagnez à tous les coups. J'ai dit : "je veux qu'ils soient condamnés, je ne veux pas de négociations". Et puis il m'a tellement roulé dans la farine ce brave homme, avec son avocat adverse qu'à un certain moment, il a dit : "il vaut mieux négocier, il vaut mieux une bonne négociation qu'un mauvais procès." (Salarié protégé, délégué syndical, membre du comité d'entreprise, 55 ans, cadre, BEPC, PME, demande de licenciement pour inaptitude) 
Avant de voir les effets que peuvent avoir ces interprétations différentielles, il faut insister sur le fait que chaque conflit (c'est-à-dire les cas où le licenciement n'a pas fait l'objet d'un accord) a un coût et que dans la plupart des cas, il est inégalement réparti. Alors que l'entreprise peut répartir la préparation du dossier sur ses services ou sur des spécialistes extérieurs qu'elle rémunère, le salarié est, la plupart du temps, seul à supporter les démarches. D'autre part, les coûts narcissiques ou identitaires sont, pour les salariés en conflit, loin d'être négligeables.

Le déclenchement du conflit ou le fait que les individus interprètent mal ce qui leur arrive ne relève donc pas tout à fait d'un choix rationnel. Cela renvoie beaucoup plus à des raisons "pulsionnelles" qui semblent échapper, comme nous le verrons, à leur volonté.

Les conséquences du conflit sont intégralement supportées par le salarié protégé qu'il s'agisse de leur devenir professionnel ou leur état psychique. Cet état dépend énormément du sens qu'ils donnent à la situation de licenciement. Ceux qui parviennent à maintenir le désaccord dans le registre d'un affrontement de positions ("le patron contre le salarié") minorent les atteintes portées à leur état mental. Au contraire ceux qui interprètent le licenciement comme une attaque personnelle semblent vivre la situation de manière beaucoup plus fragile et plus problématique.

\section{D) Les ressources sont différentes pour chaque acteur}

Chaque conflit (c'est-à-dire les cas où le licenciement n'a pas fait l'objet d'un accord, au sens sociologique du terme) a un coût qui, dans la plupart des cas, est inégalement réparti dès lors qu'existe une différence de positions: les moyens matériels mis en œuvre divergent permettant, par exemple, à l'interlocuteur patronal de donner forme au conflit et de formaliser ou d'objectiver les arguments. Le constat d'huissier par exemple, utilisé pendant une grève, semble être une pratique constante chez les employeurs qui, à l'inverse, n'est pas mobilisée par les salariés qui n'y pensent même pas. Or la "mise en forme" facilite l'administration de la preuve sans investigation supplémentaire.

Le temps constitue aussi une ressource tout à fait importante, et ce d'autant plus que les procédures sont très longues. L'inspecteur du travail fait quasiment toujours appel, dans les dossiers étudiés, à la faculté de prolonger la durée de la procédure. S'engager dans une procédure, c'est aussi, de part et d'autre, faire un pari sur la capacité à tenir le conflit dans le temps. Lorsque le salarié reste dans l'entreprise, c'est sa capacité à demeurer dans le conflit qui est déterminante. Dans d'autres cas, à certaines étapes de la procédure ${ }^{1}$, le salarié est déjà licencié ; c'est alors sa capacité à s'assurer des moyens de subsistance qui est en jeu, même si la plupart bénéficient des allocations de chômage. La situation devient extrêmement problématique lorsque l'autorisation administrative de licenciement a été refusée mais que l'entreprise a été mise en cessation de paiement, que certaines activités ont été rachetées par deux entreprises qui refusent de prendre en charge le reclassement du salarié protégé ; ce dernier n'est donc pas licencié et son contrat est maintenu (il ne peut, en théorie, prétendre aux indemnités de chômage) mais il ne réalise pas sa prestation de travail et perçoit une rémunération pour autant que l'administrateur dispose encore de liquidités. D'autre part, les délais d'une procédure contentieuse, extrêmement longs, impliquent que les parties disposent de temps. C'est certainement aussi l'une des raisons pour lesquelles les contestations sont aussi peu nombreuses. Mais pour ceux qui poursuivent, le risque de perdre est d'autant plus grand qu'ils se sont vus donner tort.

\footnotetext{
${ }^{1}$ Le recours devant le tribunal administratif n'est pas suspensif..
} 
"L'inspecteur du travail [...] accepte (le licenciement) on est (déjà) licencié. [...] Après le ministère, si on continue, on va en administratif. Ça ne sert plus à rien, on sait pertinemment que le tribunal administratif ne va jamais prendre de décisions (contre ce qui a été décidé) avant." (Salarié protégé)

Or les salariés protégés, particulièrement lorsqu'ils n'appartiennent pas à une organisation syndicale, supportent quasiment seuls les coûts du conflit. L'organisation syndicale intervient assez peu dans le procès en tant que partie à l'instance. Elle apporte cependant, pour ses adhérents, un soutien tant au niveau du conseil que de l'apport de compétences juridiques (financement d'un avocat).

Les premières ressources pour supporter le conflit sont donc d'ordre cognitif. C'est la capacité à analyser les enjeux des situations, à prendre du recul par rapport aux problèmes rencontrés. Faire appel à des ressources extérieures à l'entreprise (ressources informationnelles ou ressources juridiques) suppose des pré-requis scolaires dont tous les salariés ne disposent pas. À cet égard, et tout particulièrement quand la situation est complexe, comme en cas de dépôt de bilan, certains salariés se retrouvent totalement démunis pour comprendre les enjeux du conflit qui auront des effets sur leur propre situation. C'est le cas d'une déléguée syndicale que la demande de licenciement met dans une position très défensive et quasiment incapable d'analyser "à froid" la situation et de pouvoir argumenter. Son discours, malgré des questions orientées sur les raisons qui ont justifié son engagement dans le mandat, décrit surtout les difficultés de sa situation ${ }^{1}$. Ses difficultés, couplées à la perception d'une situation qui lui échappe, expliquent la logique de soupçons qu'elle développe et qu'elle applique à toutes les situations rencontrées professionnellement et à tous les acteurs engagés dans l'opération de restructuration.

\begin{abstract}
"(L'entreprise précédente) a été revendue par obligation, je pense qu'il y a eu un procès, on ne sait pas trop ce qui c'est passé, donc c'est la famille S. qui a repris tout ça. Cette famille reprend des entreprises, mais elle les massacre. [...] Oui, pour les liquider. [...] Tout ce qui était syndical et CE étaient complètement écartés de la promotion, mon salaire n'a pas évolué depuis 15 ans. [...] Il devait y avoir des versements d'argent d'un côté, le président du directoire avait 10 sociétés [...] En tout cas, c'était une boîte où les Anglais apportaient de l'argent et ça fuyait d'un autre côté par l'intermédiaire de je ne sais pas trop qui. [...] On a eu des réunions à n'en plus finir, on nous a baratiné. (L'administrateur) a fait un recours hiérarchique, dans ce recours, elle a écrit des sottises parce qu'elle dit que je ne fais pas partie de l'activité reprise et moi je démontre que j'en fais partie, donc, dans ce recours, elle a écrit des sottises. [...] C'est vrai que depuis le départ du dossier, le service juridique a traité le dossier par-dessus la jambe. S'il n'y avait pas eu un recours juridique, j'aurai été virée comme une malpropre.» (Salariée protégée, juge prud'hommes, syndiquée, 54 ans, agent de maîtrise, CAP, PME, demande de licenciement pour inaptitude)
\end{abstract}

Or tant son niveau de formation de base (CAP de couture) que son parcours dans une même entreprise (26 ans d'ancienneté), comme le type de carrière qui lui a été offert (nommée agent de maîtrise à l'ancienneté) ne lui fournissent pas les capacités cognitives suffisantes pour affronter la situation. Elle n'arrive pas non plus à utiliser les apprentissages acquis lors des cours de droit du travail suivis en cours du soir au CNAM. En effet, le danger, l'extrême urgence ou les problèmes graves ont souvent tendance à empêcher la mobilisation de ce type de capacités. En nous forçant à nous polariser sur les problèmes, en nous empêchant de prendre du recul, ces situations nous paralysent et occultent nos capacités de raisonnement.

\footnotetext{
${ }^{1}$ L'entretien cité dans cette partie a été très éprouvant. Il a été interrompu en cours car la salariée s'est progressivement effondrée au fur et à mesure qu'elle revivait les difficultés rencontrées et que l'issue lui paraissait de plus en plus problématique.
} 
Des individus très diplômés, très qualifiés confrontés à un licenciement qu'ils n'avaient jamais envisagé peuvent ainsi perdre tout ressort et se laisser dériver. On peut en avoir un aperçu assez parlant dans le film de Jugnot "une époque formidable" ou mieux encore dans celui de Laurent Cantet "L'emploi du temps".

Il faut ici insister sur l'extrême fragilité qui peut faire suite à certains licenciements visant des salariés qui se trouvent totalement démunis parce qu'ils ne disposent pas des ressources nécessaires (soit de manière structurelle ou plus rarement comme décrit dans l'exemple précité de manière conjoncturelle) pour affronter ce problème. Les cadres et les ouvriers ne sont pas en situation d'égalité. On peut aussi en avoir une indication en comparant les justifications des licenciements des ouvriers et des cadres telles qu'elles apparaissent dans les dossiers de l'inspection du travail. Pour les ouvriers qui se trouvent, sauf cas particulier, en position de déficit de ressources sémantiques et cognitives, le registre des licenciements pour fautes professionnelles se construit sur une argumentation particulière. Dans ces dossiers, la les situations décrites font souvent référence à des phénomènes de violence : de l'injure à l'intimidation puis aux coups. Or ce registre ne fait que refléter la difficulté qu'ont les salariés dans les fonctions les plus manuelles à répondre aux pressions ou aux attaques de la hiérarchie qui se déroulent, elles, sur un registre purement verbal et policé, mais qui peuvent être d'une très grande brutalité. Face à une violence qui se dissimule derrière des tournures édulcorées, euphémisée par la référence à des impératifs de gestion par exemple, certains salariés ne trouvent comme moyen de réponse que la violence physique. La violence peut alors être analysée comme une tentative désespérée de répondre à des situations vécues sur le registre de la plus grande injustice .

Quand on analyse les dossiers de licenciement de cadres, le registre sémantique présent dans les dossiers ne se réfère plus à la violence mais à la compétence. Les entreprises reprochent aux salariés leur manque de compétence. De manière symétrique, les salariés rétorquent en remettant en cause la compétence des décisionnaires, leur capacité à gérer les dysfonctionnements, à faire des diagnostics pertinents, à élaborer des stratégies industrielles et commerciales adéquates. Le deuxième registre apparaît socialement plus "acceptable" et surtout peut donner lieu à des argumentations et des interprétations divergentes et, donc, sont beaucoup plus facilement contestables devant l'inspecteur du travail ou devant le Ministère.

Il est, d'autre part, important de signaler que dans les dossiers, et notamment les moins étoffés, la position du salarié ne fait pas toujours l'objet d'une formalisation. De même que la présence du salarié dans la procédure, l'écrit est étroitement dépendant du niveau de capital culturel détenu. Les inégalités des acteurs dans la procédure renvoient donc, en premier lieu, à des inégalités cognitives.

Toutefois, même pour des individus fortement dotés en capital scolaire, des situations ou des problèmes comme le sentiment de danger dans l'entreprise, le licenciement ou la permanence du chômage peuvent en quelque sorte neutraliser une grande part des ressources dont dispose l'individu (Correia, Pottier, 2001). Dans ce cas, le problème rencontré déshabille l'individu de tous ses attributs, le laissant seul et démuni face à des situations qu'il ne comprend pas et qu'il ne parvient pas à maîtriser.

Dans le processus conflictuel, le cadre légal est aussi utilisé par l'entreprise et le salarié (les parties à la procédure) comme une ressource. C'est en fonction de ce rapport de force juridique que se construit l'issue du conflit. 
L'analyse des entretiens montre que le droit apparaît comme un moyen d'élargir la légitimité de l'argumentaire, en particulier en cas de désaccord.

«Là, ça a commencé parce qu'à la $1^{\text {ère }}$ réunion du $\mathrm{CE}, \mathrm{X}$ m'a insulté parce qu'à chaque fois qu'il disait quelque chose, je lui répondais en disant que ça ne fonctionnait pas comme ça. Je m'étais beaucoup formé entre temps. Il avait eu l'habitude d'un CE à sa botte, il a donc été très surpris. À la $2^{\text {ème }}$ réunion, il est arrivé avec un code du travail, et il faisait semblant de le feuilleter à chaque fois, il voulait, pour vous citer un exemple, rédiger lui-même les PV de CE ! la $1{ }^{\text {ère }}$ rédaction, faite par Y et moi, une fois qu'on lui a présenté, il a voulu faire son PV, alors on lui a dit que ça ne marchait comme ça. Bref, on discutait sur des points de détail, des phrases, on a concédé $50 \%$ du texte, mais le reste on ne voulait plus. Et là il me sort, on était tous pantois : Monsieur Z, vous êtes un enculeur de mouche.» (Salarié protégé, délégué syndical, cadre, 42 ans, Bac, PME, demande de licenciement pour faute)

Cette place accordée au droit se traduit par ce que l'on pourrait nommer une "juridicisation de la fonction".

«Je tiens par exemple des permanences une fois par semaine depuis plusieurs années [...] C'est vrai, quand les gens viennent nous voir, ils ont des demandes précises et de plus en plus précises, ils sont très demandeurs dans leurs précisions. Maintenant les gars ciblent et sont précis dans leurs attentes, donc ça demande que nous aussi on soit plus pointus, plus compétents, donc effectivement c'est une remise en cause constante.» (Salarié, juge prud'homal, délégué syndical, ouvrier, 58 ans, CEP, TPE, demande de licenciement pour inaptitude)

Le droit est alors principalement appréhendé dans sa dimension instrumentale : il est une ressource, dès lors que le salarié est en capacité de la mobiliser.

«J'ai commencé à constituer mon dossier, parce que je me suis dit, "un jour on va aller au clash." Alors, voici un exemple : je fais une demande écrite pour pouvoir aller à tel salon, on me répondait : "avez-vous un intérêt quelconque à aller dans ce salon ?" Je faisais la personne qui acceptait tout en sachant que je préparais mon dossier, et le jour où je l'ai sorti, ils sont tombés de très haut.» (Salarié protégé, délégué syndical, membre du comité d'entreprise, 55 ans, cadre, BEPC, PME, demande de licenciement pour inaptitude)

Dans son expression la plus "ultime", c'est-à-dire le procès, le droit prolonge, par d'autres moyens, le désaccord ou le conflit.

«Comme on avait déjà engagé une action prud'homale où on veut aller jusqu'au bout, je veux aller au moins jusqu'au bout de ça avant de partir. Et par principe, parce que je sais que ça les emmerde, et bien ça me fait plaisir de rester là. Parce qu'ils nous ont tellement fait chier pendant un an et demi, ils ont essayé de me virer, ils n'y sont pas arrivés, ils vont sûrement recommencer. Mais à mon avis, ça va être de plus en plus difficile, ils vont avoir du mal à trouver des motifs. Parce que s'ils n'en ont pas trouvé jusque maintenant des motifs, parce que comme vous le disiez, ce n'est pas très compliqué de virer quelqu'un. Mais s'ils n'en ont pas trouvé pour me virer, c'est qu'ils vont sans doute avoir du mal à me virer» (Salarié protégé, membre du comité d'entreprise, délégué du personnel, syndiqué, 37 ans, cadre, Bac+2, PME, demande de licenciement pour faute)

On ne peut alors qu'être amené à s'interroger sur le sens de ces évolutions. Le recours au droit ne pose-t-il pas la question de la pertinence du recours à la grève, qui, pour un secrétaire de CE interrogé

«N'est pas adapté à notre temps.» (Secrétaire comité d'entreprise, syndiqué, 58 ans, PME) 
Ce constat va dans le sens de celui que dresse Alain Supiot ${ }^{1}$ pour qui «la grève était tout d'abord une arme adaptée au modèle dominant de l'entreprise industrielle, conçue comme une institution soumettant une collectivité de travailleurs au pouvoir d'un employeur sous l'égide d'une loi nationale. [...] Ainsi conçu, le droit de grève a joué depuis un siècle un rôle majeur dans l'édification de l'Etat-Providence. [...]. Mais aujourd'hui le cadre économique et juridique dans lequel s'est affirmé le droit de grève a changé. Le modèle de la grande entreprise industrielle n'a cessé de décliner en Europe depuis 20 ans, au fur et à mesure que les entrepreneurs se sont affranchis des frontières nationales et que les Etats ont été en revanche soumis aux règles du commerce international. Face à ces changements, le droit de grève est une arme de moins en moins bien adaptée».

Le droit apparaît ainsi particulièrement adapté à une population salariée de plus en plus diplômée et qualifiée. Le rapport de forces quantitatif et physique des premiers temps du syndicalisme tend alors à être remplacé par des affrontements de compétences ou le droit peut être mobilisé quasiment à parité par tous les protagonistes. Toutefois cet usage du droit ne doit pas faire oublier que la « juridicisation » des relations suppose que les individus disposent déjà de capacités cognitives. Remplacer le rapport de forces par des argumentations juridiques revient alors à exclure du jeu social tous ceux qui n'ont pas accès à ce niveau d'argumentation.

En effet, si tout le monde semble capable de lire un texte de loi, la maîtrise des procédures et des formes juridiques relève d'un autre niveau de compétences.

"Il a décidé d'aller tout seul aux prud'hommes, sans prendre d'avocat [...] Là, il n'a pas pesé lourd : l'avocat l'a démonté, il n'avait rien chiffré, il avait fait ça sur un cahier d'écolier." (Salarié protégé)

Toutefois, cette difficulté ne joue pas toujours en défaveur des salariés. Dans les petites entreprises familiales, par exemple, les salariés les mieux formés peuvent utiliser cette ressource pour rééquilibrer les positions entre subordonnés et direction.

\begin{abstract}
"Moi je me suis mis sur le droit social parce que j'étais très persévérant.[...]. Et à partir de là, ça a commencé à jouer sec.» (Salarié protégé, délégué syndical, cadre, 42 ans, Bac, PME, demande de licenciement pour faute)

" La seule chose où il n'a pas fait d'effort, c'est de se former en tant que président de CE, et ça, ça a été sa faille, parce qu'à chaque fois, même sur des points de détail, je pouvais moi. Avec mes heures de délégation CE, DP, délégué syndical j’étais informé. [...] Ce qui l'a vraiment excédé, c'est de se rendre compte qu'il était dépassé.» (Salarié protégé, délégué syndical, cadre, 42 ans, Bac, PME, demande de licenciement pour faute)
\end{abstract}

Cette arme est cependant à double tranchant : en entraînant le conflit sur le versant purement juridique et en multipliant les affrontements, les salariés protégés prennent le risque de se perdre dans une croisade judiciaire qu'ils mènent seuls, séparés des autres salariés de l'entreprise.

"Je pense qu'ils nous soutenaient en principe, mais dès qu'il fallait être sur le terrain, ils n'étaient
plus là. C'est difficile parce que comme l'ensemble des magasins sont éloignés les uns des autres.
Mais il est sûr qu'à un moment les gens se sont lassés, parce que comme on a fait une bagarre qui
était un peu trop juridique et qu'on n'était pas assez proche d'eux, je pense qu'ils nous ont un peu
lâchés. Alors que ce n'était pas ça, nous à la base, on voulait que la société fonctionne
normalement au niveau du CE. Mais on devait passer par le juridique, on a dû se battre sur tout

${ }^{1}$ A. Supiot, "Revisiter les droits d'action collective", Dr. Soc. 2001, p. 687 à 704, p. 688 et 689. 
pour avoir l'argent.» (Salarié protégé, membre du comité d'entreprise, délégué du personnel, syndiqué, 37 ans, cadre, Bac+2, PME, demande de licenciement pour faute)

On arrive ainsi à des victoires paradoxales : à chaque coup gagné sur l'adversaire, on donne l'impression d'un conflit de plus en plus étranger aux intérêts des salariés et l'on finit par perdre leur soutien.

\section{E) Le droit une ressource au service des personnes en cas de conflit}

Le droit possède, dans cette configuration, un statut particulier. En situation normale, c'est-àdire quand le salarié se consacre à son activité et que l'entreprise semble lui en être reconnaissante, le droit ne peut être mobilisé. En effet, son usage est contraire au principe de confiance qui préside aux relations. D'autre part, l'application de règles juridiques aurait pour effet de restreindre l'investissement des salariés, alors que c'est ce qui rend intéressant leur activité.

En effet, l'utilisation du droit est souvent une manière de marquer les frontières de son pouvoir.

\footnotetext{
"Je connaissais bien mes droits par rapport à la formation." (Salarié protégé, membre du comité d'entreprise et du CHSCT, syndiqué, agent de maîtrise, 28 ans, BTS, PME, demande de licenciement pour inaptitude)
}

Dans ce modèle, l'investissement ne peut se cantonner aux obligations contractuelles, ne serait ce que parce que le fait de dépasser ce seuil est, en lui-même, un paiement. En effet, les profits de l'investissement sont inclus dans le fait même de s'investir (lire nos développements antérieurs sur la recherche d'une valorisation personnelle).

La référence à des règles ou à des procédures juridiques ne survient donc que quand la relation salarié - entreprise devient instable.

Le mandat de conseiller prud'homal constitue un cas particulier. Il semble bénéficier d'une certaine "aura" qui fait aussi parfois s'éloigner les conseillers d'un mandat qui demeure syndical ${ }^{1}$. Les conseillers prud'homaux sont des juges ; à ce titre, ils "disent le droit" et leurs décisions s'imposent aux parties au procès. Le mandat qu'ils occupent leur impose de maîtriser le droit. Certains y parviennent de manière magistrale. Le mandat constitue alors aussi une promotion sociale pour des personnes qui ont bien souvent arrêté très tôt leurs études.

Ce mandat apparaît aussi plus consensuel, raison pour laquelle on l'a rattaché à la personnalisation du mandat : il s'exerce en dehors de l'entreprise et des enjeux sociaux immédiats, ainsi que l'explique un Conseiller prud'homme.

"(Est ce que le fait que vous soyez conseiller prud'homal a eu des effets sur votre travail ?) Non, au
contraire, de la part de mon directeur, c'était plutôt une référence en fait. [L'employeur] s'est servi
de moi en se disant M. F. est conseillé prud'homal donc il représente les lois, il représente la
justice. Donc au sein de l'entreprise, il ressentait ça comme un bien-être. Il disait à partir du

${ }^{1}$ Ces observations sont le fruit de sept ans de formation de conseillers prud'homaux au sein de l'ISST. 
moment où on a un représentant de la loi dans l'entreprise, on est bien, ça veut dire que nous, la loi, on la respecte. C'est vrai qu'à la limite, il la respectait." (Salarié, juge prud'homal, délégué syndical, ouvrier, 58 ans, CEP, TPE, demande de licenciement pour inaptitude)

\section{III- La fin du conflit}

L'analyse de certains entretiens met au jour une utilisation dévoyée de la règle de droit qui se trouve ainsi détournée ${ }^{1}$ de ses finalités et, ce faisant, des fonctions qui lui avaient été assignées par le législateur lors de l'élaboration des textes. Deux exemples peuvent être donnés: l'inaptitude, qui se construit avec le salarié (A), et les transactions, habillage juridique qui couvre, contra legem, l'existence d'un départ négocié entre le salarié protégé et l'entreprise (B).

Mais il faut, en préalable, essayer de comprendre ces phénomènes : en effet, l'inaptitude ou la transaction ne sont pas toujours ni recherchées ni acceptées avec emphase par les salariés. Elles sont aussi un pis-aller, qui permet de mettre à distance, au moins pour un temps, des situations que l'on a le plus grand mal à supporter.

"J'en étais à un point où je ne dormais plus, j'étais incapable de travailler. Pour pouvoir se battre, il
faut aussi être performant dans son travail, il faut pouvoir être irréprochable. Moi je considère que
je n'étais plus en mesure de l'être." (Salarié protégé, membre comité d'entreprise, délégué du
personnel, non-syndiqué, 29 ans, cadre, Bac+2, PME, demande de licenciement économique)

Les effets d'un conflit de longue durée sont loin d'être anodins pour les individus, surtout qu'ils se vivent souvent sur la base d'une inégalité de ressources dont les effets sont dévastateurs sur leur personnalité.

"Le salarié arrive, il est déjà sous traitement médical depuis des mois, il s'effondre en larmes dans votre bureau, il nous supplie quasiment à genoux de le laisser partir. [...] Je réalise aussi ce que ça peut signifier pour les salariés du privé d'être face à des phénomènes de harcèlement qui peuvent être incessants, et qui peuvent durer des années, qui foutent en l'air leur vie privée, familiale, etc... Encore une fois, tous les représentants du personnel n'en sont pas là heureusement, mais parfois ça peut être ça." (Inspecteur du travail)

C'est en grande partie pour ces raisons que des inspecteurs du travail peuvent autoriser les licenciements "convenus" car ils font preuve d'empathie par rapport au sort des salariés.

"J'ai parfaitement conscience sur ce que je vous disais tout à l'heure, en laissant partir le salarié
qui en a pris plein la figure et qui ne souhaite plus qu'une chose, c'est s'en aller et quitter
l'entreprise. J'ai parfaitement conscience aussi que le chèque de l'employeur va acheter son départ
et achète aussi la décision de l'inspecteur, c'est pour ça aussi que ce n'est absolument pas
satisfaisant. Et ça je le mets dans la balance aussi, je sais aussi que je me fais rouler d'une certaine
façon, puisqu'il suffit d'un chèque pour acheter la décision de l'autorisation de l'inspecteur. C'est-
à-dire que le salarié vient vous dire : je n'en peux plus, je veux partir, alors vous comprenez qu'il y
a eu une transaction. Mais si en même temps, j'ai vraiment conscience que c'est ça ou le
condamner à des mois épouvantables qu'il a déjà vécus et en ajouter d'autres." (Inspecteur du
travail)

\footnotetext{
${ }^{1}$ Le verbe "dévoyer" est tiré de desvoier, qui signifie, en son sens littéral tel qu'apparu au XIIe siècle, "pousser hors de la voie". A. Rey, Dictionnaire historique de la langue française, éd. Le Robert, 1995.
} 
En effet, la principale raison pour laquelle les salariés acceptent des transactions ou demandent leur licenciement pour inaptitude, c'est parce que le licenciement permet de sortir d'une situation de crise, parfois totalement insoutenable pour le salarié, tout en gardant le bénéfice des allocations chômage.

Il est probable que si la démission d'un salarié protégé lui offrait les mêmes garanties, le nombre de licenciements subirait une baisse au profit des démissions qui, elles ne dépendent pas de la décision de l'inspecteur du travail.

\begin{abstract}
"J'ai un certain nombre de cas de licenciement de représentants du personnel où on se serait normalement situé dans le cadre d'une démission mais où des salariés, parce qu'ils sont représentants du personnel, essaient de profiter de cette opportunité pour négocier un départ sous forme de licenciement ou carrément commettre une faute, de type absence injustifiée, ne se présentent plus au travail et disent: "je ne me présente plus parce que je souhaite être licencié." "Pourquoi vous souhaitez être licencié ?" "Parce que je souhaite toucher les ASSEDIC." (Inspecteur du travail)
\end{abstract}

\title{
A) L'inaptitude impose la rupture.
}

De la même manière, l'enquête a montré que le constat d'une inaptitude (réelle) au travail donnait la plupart du temps lieu à l'acceptation de la situation par toutes les parties. L'inaptitude réfère peu ou prou à la même logique de contrainte externe. Elle s'impose parfois autant à l'entreprise qu'au salarié.

"Je ne peux pas reprendre mon poste dans l'entreprise, ni un autre poste dans cette entreprise.
Toutes les procédures ont été respectées d'une très bonne manière. [...] C'est moi qui l'ai demandé
bien sûr, puisque j'avais des problèmes de maladie. C'est la médecine du travail qui, au bout de
deux ans et demi a fini par me reconnaître inapte à tous les postes dans l'entreprise, donc le patron
a été obligé de me licencier, mais il a respecté la procédure.» (Salarié, juge prud'homal, délégué
syndical, ouvrier, 58 ans, CEP, TPE, demande de licenciement pour inaptitude)

L'inspecteur du travail peut alors difficilement refuser l'autorisation quand le salarié protégé manifeste un tel désir à se faire licencier.

"J'ai envoyé un courrier (à l'inspecteur du travail) en disant que j'acceptais mon licenciement, parce qu'à 57 ans... Je ne pouvais pas faire autrement que d'accepter mon licenciement. Mais j'avoue sincèrement que j'étais content de mon licenciement, il faut être honnête, j'étais content de mon licenciement parce que j'avais quand même 42 ans de travail.» (Salarié, juge prud'homal, délégué syndical, ouvrier, 58 ans, CEP, TPE, demande de licenciement pour inaptitude)

Le cas d'inaptitude présenté dans cette partie ne rend pas compte de la complexité des jeux qui se nouent autour des licenciements pour inaptitude qui sont bien souvent une co-construction plus ou moins conflictuelle entre l'entreprise, le médecin du travail et le salarié.

De manière plus générale, les cas présentés ici paraissent un peu idylliques et rendent faiblement compte de la tension qui entoure toutes les demandes de licenciement. Il existe, par exemple, une «zone d'ombre » qui entoure les licenciements pour motif personnel. Ainsi que l'expliquent Maria-Teresa Pignoni et Patrick Zouary, « les circonstances et les raisons de l'évolution des licenciements pour motif personnel ${ }^{1}$ restent largement méconnues. [...]

\footnotetext{
${ }^{1}$ Notre champ n'est pas celui du licenciement personnel, mais les cas de licenciement collectifs dans lesquels les choix ne se font pas sur des critères personnels posent peu de problèmes : le salarié protégé s'inscrit "naturellement" dans un traitement
} 
Sanction d'une faute ou d'une insuffisance professionnelles, démission déguisée ou licenciement négocié... Le licenciement autre qu'économique est difficile à cerner, et la catégorie administrative et statistique "autres licenciements" ne rend pas compte de l'hétérogénéité des situations qui en sont à l'origine ${ }^{1}$. Cette zone d'ombre apparaît d'ailleurs d'autant plus problématique que les entrées à l'ANPE suite à un licenciement pour motif personnel sont deux fois plus nombreuses que celles qui font suite à un licenciement économique ${ }^{2}$.

\title{
B) L'inaptitude se construit avec le salarié
}

Pour certains salariés, la reconnaissance d'une inaptitude par le médecin du travail constitue l'un des moyens de se retirer d'une situation de travail tout en ne démissionnant pas (1). L'avis d'inaptitude est ainsi utilisé suivant d'autres finalités que celles pour lesquelles il a été mis en place, provoquant, de ce fait, une distorsion de la règle de droit (2).

\section{1) Un moyen pour le salarié protégé de se retirer d'une situation de travail}

Les entretiens réalisés montrent que l'inaptitude est parfois demandée par le salarié qui, tout en n'étant pas licencié, souhaite cependant quitter l'entreprise.

\begin{abstract}
"Je savais que je ne pouvais pas être licencié mais c'était des conditions de travail... Disons que je me suis toujours donné à fond dans mon travail, ça c'est toujours bien passé, et j'ai envie d'aller au travail en sachant qu'on apprécie mon travail. Â partir de là, c'est vrai que ça a commencé à me travailler, et je me suis dit qu'il fallait que je parte, je savais que je ne pouvais pas partir tout simplement comme ça, c'était assez difficile étant donné qu'on était protégé. Ça faisait aussi un moment que la médecine du travail me disait que je commençais à avoir des problèmes respiratoires. Donc je me suis dit que j'allais utiliser ce moyen pour partir pour inaptitude médicale, on a donc commencé à monter un dossier, mais il était déjà bien entamé bien avant ça. Je suis allé le voir et je lui ai dit : vous m'avez dit que je commençais à avoir un petit problème qui commence à apparaître, donc on a commencé à monter un dossier de notre côté avec la médecine du travail, qui était réel donc ça m'arrangeait bien. J'ai vu aussi des pneumologues qui l'ont prouvé, parce qu'ils m'ont arrêté pendant une semaine, donc ces problèmes-là ont disparu au bout d'une semaine, je n'avais plus de sifflements, on m'a fait un traitement. Ils ont montré par des diagrammes et des tests respiratoires que ça avait quand même diminué de $50 \%$, au bout d'une semaine." (Salarié protégé, membre du comité d'entreprise et du CHSCT, syndiqué, agent de maîtrise, 28 ans, BTS, PME, demande de licenciement pour inaptitude)
\end{abstract}

L'utilisation de l'inaptitude peut d'ailleurs être une stratégie de l'avocat plus que du salarié, ainsi que l'illustrent les propos d'un salarié protégé :

"[L'avocat] me dit : il faut négocier Monsieur P., on a toutes les chances de gagner, mais une
bonne négociation, c'est mieux qu'un mauvais procès, vous savez le mot traditionnel qu'ils ont
dans leur truc. (Mais il vous dit en même temps que le licenciement n'est pas possible.) Alors
voilà, c'est là que la combine rentre en cause. Alors on me dit : "vous savez par rapport à votre
épaule, on peut trouver une inaptitude, etc..." J'ai répondu : "moi je vais vous dire que je suis
inapte, mais vous êtes fous ?" (Il m'a dit : "mais non, on va mettre dans le coup le médecin du

collectif pour lequel son statut ne souffre pas de discrimination. Par contre les licenciements collectifs conflictuels dans notre enquête se construisent souvent par le fait que le licenciement recherché est un licenciement individuel (celui du salarié protégé) que l'on essaye d'habiller d'oripeaux collectifs.

${ }^{1}$ Pignoni M-T., Zouary P. (2003) "Les nouveaux usages du licenciement pour motif personnel", Premières informations, juillet $2003 \mathrm{n}^{\circ} 28.2$.

${ }^{2}$ Ibid. 
travail, vous verrez, vous vous faites faire un mot par votre médecin." Donc ils m'ont montré un truc, j'ai dit: "mais c'est une aberration ce truc. Il faut que j'aille voir mon médecin pour qu'il me fasse un mot comme quoi je dois passer une visite médicale, enfin..." [...] Alors, moi, j'ai dit : "mais attendez, je ne suis pas inapte". Alors sur ce, on prend rendez-vous pour moi avec le médecin du travail. Première visite, il me dit : "je veux quand même bien vous voir pour voir si vous êtes prêt dans votre tête, enfin, machin et baratin, et puis c'est parti comme ça." (Salarié protégé, délégué syndical, membre du comité d'entreprise, 55 ans, cadre, BEPC, PME, demande de licenciement pour inaptitude)

Le dirigeant peut d'ailleurs "adhérer implicitement" à cet usage dévoyé du droit en en ayant la connaissance, ainsi que l'exprime un Directeur Général.

"Je pense qu'il [le salarié] a saisi le motif médical pour faire une sortie de la boîte" (Directeur général, PME)

Mais dans les deux cas de figure rencontrés, l'inaptitude ne se construit pas ad hoc; il existe préalablement un problème de santé qui cependant n'impliquerait pas une reconnaissance d'inaptitude.

"Il y a eu un dossier d'inaptitude qui a été monté, ils se sont quand même référés à quelque chose d'important puisque c'était les suites de mon opération, de mon accident. Ça n'a pas été quelque chose d'inventé de toutes pièces, ça a été quand même construit par rapport à un élément. (Mais le médecin du travail savait, il connaissait la situation, que vous vouliez partir ?) À votre avis, le médecin du travail est payé par qui ? C'est l'entreprise qui le paie. Ils sont toujours favorables quand il y a des problèmes de sécurité, mais ce sont des gens dont je me méfie comme de la peste." (Salarié protégé, délégué syndical, membre du comité d'entreprise, 55 ans, cadre, BEPC, PME, demande de licenciement pour inaptitude)

Or l'usage qui est fait de l'avis d'inaptitude comme moyen de retrait d'une situation de travail pose le problème du décalage entre les finalités de la règle et les pratiques.

\section{2) La distorsion de la règle de droit}

L'article L. 122-45 interdit à l'employeur, à peine de nullité, de licencier un salarié en raison de son état de santé, sauf inaptitude constatée par le médecin du travail. Si la maladie entraîne la suspension du contrat de travail ${ }^{2}$, la reprise de son activité professionnelle par le salarié est soumise à une visite ${ }^{3}$ réalisée par le médecin du travail, et durant laquelle ce dernier peut le

\footnotetext{
${ }^{1}$ «Aucune personne ne peut être écartée d'une procédure de recrutement, aucun salarié ne peut être sanctionné ou licencié en raison de son origine, de son sexe, de ses mœurs, de sa situation de famille, de son appartenance à une ethnie, une nation ou une race, de ses opinions politiques, de ses activités syndicales ou mutualistes, de ses convictions religieuses ou, sauf inaptitude constatée par le médecin du travail dans le cadre du Titre IV du livre II du présent code, en raison de son état de santé ou de son handicap».

${ }^{2}$ Art. L. 122-24-4 pour la maladie et l'accident "ordinaire" et L. 122-31-1 s'agissant de l'accident du travail et de la maladie professionnelle.

${ }^{3}$ Art. R. 245-51 : «Les salariés doivent bénéficier d'un examen par le médecin du travail après une absence pour cause de maladie professionnelle, après un congé de maternité, après une absence d'au moins huit jours pour cause d'accident du travail, après une absence d'au moins vingt et un jours pour cause de maladie ou d'accident non professionnel et en cas d'absences répétées pour raisons de santé.

Cet examen a pour seul objet d'apprécier l'aptitude de l'intéressé à reprendre son ancien emploi, la nécessité d'une adaptation des conditions de travail ou d'une réadaptation du salarié ou éventuellement de l'une ou de l'autre de ces mesures.

Cet examen doit avoir lieu lors de la reprise du travail et au plus tard dans un délai de huit jours.

Cependant, à l'initiative du salarié, du médecin traitant ou du médecin conseil des organismes de sécurité sociale, lorsqu'une modification de l'aptitude au travail est prévisible, un examen peut être sollicité préalablement à la reprise du travail, en vue de faciliter la recherche des mesures nécessaires. L'avis du médecin du travail devra être sollicité à nouveau lors de la reprise effective de l'activité professionnelle.

Le médecin du travail doit être informé de tout arrêt de travail d'une durée inférieure à huit jours pour cause d'accident du travail afin de pouvoir apprécier, notamment, l'opportunité d'un nouvel examen médical»
} 
déclarer inapte. L'avis d'inaptitude qui peut en résulter met un terme à une situation dans laquelle le salarié ne remplit plus ses obligations, étant dans l'impossibilité de travailler. Ce terme ne peut cependant intervenir qu'après une procédure contraignante qui vise à maintenir le salarié dans l'emploi. Le Code du travail prévoit en effet que le salarié reconnu inapte à l'emploi qu'il occupait doit se voir proposer un autre emploi "approprié à ses capacités, compte tenu des conclusions écrites du médecin du travail et des indications qu'il formule sur l'aptitude du salarié à exercer l'une des tâches existantes dans l'entreprise..." (art. L. 122-244). La reconnaissance de l'inaptitude réalisée par le biais de l'avis ne peut donc intervenir qu'en dernier lieu, après que le reclassement a été recherché, et suivant une procédure qui confère au médecin du travail un rôle fondamental. Suivant l'article R. 241-51-1, le constat d'inaptitude ne peut intervenir qu'en respectant deux conditions cumulatives :

- une étude du poste et des conditions de travail dans l'entreprise

- deux examens médicaux de l'intéressé espacés de deux semaines.

Pour ce faire, le médecin dispose de larges prérogatives: il peut prescrire des examens complémentaires (art. R. 241-52). Ils doivent se dérouler pendant le temps de travail et donc donner lieu au maintien du salaire (R. 241-53). Il dispose, s'agissant de la recherche du reclassement du salarié, d'un pouvoir de proposition (mutation ou transformation de poste ${ }^{1}$ ). La recherche des possibilités de reclassement doit d'abord s'apprécier au sein de l'entreprise ${ }^{2}$, dans emploi qui doit, suivant l'article L. 122-32-5 du Code du travail, être aussi comparable que possible à celui précédemment occupé ${ }^{3}$; puis, à défaut, à l'intérieur du groupe auquel appartient l'employeur, et, précise la Cour de cassation, «parmi les entreprises dont les activités, l'organisation ou le lieu d'exploitation lui permettent d'effectuer la mutation de tout ou partie du personnel» ${ }^{4}$. Ce n'est qu'au terme de cette procédure que l'employeur pourra procéder au licenciement du salarié inapte non reclassé.

Le salarié absent pour maladie ou accident bénéficie donc d'une protection juridique renforcée ; il entre dans une catégorie juridique particulière. Cette protection se réalise essentiellement par le respect d'une procédure contraignante. Cette procédure s'impose aux parties au contrat, quand bien même le salarié aurait implicitement refusé l'offre de reclassement ${ }^{5}$ ou que l'inaptitude ait été reconnue pour tout poste de travail. Le Code du travail consacre ainsi l'indisponibilité du statut et, plus largement, de la règle de droit qui échappe à la volonté des parties, même si un récent arrêt de la Cour de cassation semble quelque peu infléchir ce raisonnement ${ }^{6}$.

\footnotetext{
${ }^{1}$ Soc. 14/01/97, Mme Petriacq c/ Sté Sogara, Dr. soc. 1997, p. 313.

${ }^{2}$ CE 30 avril 1997, $n^{\circ} 158474$, Perre.

${ }^{3}$ Un emploi à temps partiel ne répond pas à ces conditions dès lors que l'employeur n'apporte pas la preuve de l'impossibilité dans laquelle il se trouvait de reclasser le salarié protégé inapte à temps plein "équivalent à celui qu'il occupait précédemment et compatible avec ses aptitudes physiques, en procédant au besoin à des mutations de personnel ou à un aménagement de certains postes de travail, ainsi que le prévoit l'article L. 122-32-5 du code du travail ; que dès lors l'inspecteur du travail était tenu de refuser le licenciement de M. Jereri" ; CE n 171476, 15 juin 1998, Onet Propreté.

${ }^{4}$ Cass. soc. 18 avril 2000, Cury c/ SA Miko ; Conseil d'Etat, $N^{\circ} 157169,9$ décembre 1998, M. Courtelessis

${ }^{5}$ Cass. soc. 16 fév. 1999, Bull. civ. V, no 76

${ }^{6}$ Cour de Cassation, Chambre sociale, 16 mars 2005, Carcoop France, D. 2005, p. 1613, note J. Mouly ; Semaine soc. Lamy 29 mars 2005, n 1208, p. 13, obs. F. Duquesne. Lire également, l'article de J. Mouly, "'Coup de vent' sur le statut des salariés protégés, Dr. Soc. sept.-oct. 2005, p. 861 à $865 ;$ Ph. Waquet, «Coup de vent sur la jurisprudence Perrier », RJS $6 / 05$, p. 419. L'arrêt ouvre la possibilité au salarié dit «protégé » de demander au juge la résiliation de son contrat de travail : «Mais attendu que si la procédure de licenciement du salarié représentant du personnel est d'ordre public, ce salarié ne peut être privé de la possibilité de poursuivre la résiliation judiciaire de son contrat de travail aux torts de l'employeur en cas de manquement, par ce dernier, à ses obligations ». Mais la résiliation prononcée aux torts de l'employeur produira les effets d'un licenciement sans cause réelle et sérieuse : l'initiative de la rupture incombe au salarié mais elle est imputable à l'employeur qui devra en supporter les conséquences financières.
} 


\section{Cass. Soc. 29 juin $1999-\mathrm{N}^{\circ} 304$ \\ Extraits}

"Les dispositions des articles L. 122-24-4 et L. 122-32-5 du Code du travail qui prévoient, notamment, que si, après une déclaration d'inaptitude prononcée par le médecin du Travail à l'issue des périodes de suspension du contrat de travail, le salarié n'est pas reclassé dans l'entreprise à l'issue d'un délai d'un mois, ou s'il n'est pas licencié, l'employeur est tenu de reprendre le paiement du salaire, excluent dans cette hypothèse la possibilité pour les parties de signer une rupture d'un commun accord du contrat de travail qui aurait pour effet d'éluder ces obligations ${ }^{1}$."

Or, l'utilisation de l'inaptitude comme moyen de se retirer d'une situation de travail, loin d'être épisodique (suivant les témoignages de médecins du travail ${ }^{2}$ ) constitue un détournement du droit (sans qu'un jugement soit porté sur la légitimité de cette utilisation). On ne peut, ce faisant, qu'être amené à s'interroger sur les raisons de cette distorsion. Les récentes enquêtes montrent de manière plus globale que les salariés font état d'une augmentation de la charge mentale au travail ${ }^{3}$ : sentiment de responsabilité, urgence, bruit, attention soutenue, interruptions, moyens insuffisants ou relations tendues. C'est ainsi que 60 $\%$ des salariés déclarent craindre des sanctions en cas d'erreur de leur part, et notamment sur leur emploi. 30\% vivent des relations de tensions avec leur hiérarchie. L'enquête communautaire menée par la Fondation de Dublin en $1996^{4}$, montre que si $9 \%$ des travailleurs déclarent être l'objet d'intimidations, le stress fait partie des problèmes de santé les plus souvent signalés (28\% des travailleurs) derrière les douleurs dorsales $(30 \%)$ et devant les douleurs musculaires dans les bras et jambes (17\% des travailleurs) ${ }^{5}$.

Les salariés protégés traduisent, en "construisant" leur inaptitude, l'existence d'un certain mal-être au travail qui peut également être révélateur de l'amoindrissement du rôle d'écran que joue le mandat. Plus exposés au conflit avec l'employeur voire les salariés ${ }^{6}$, les salariés protégés seraient aussi plus fragilisés du fait d'un glissement des pressions exercées du mandat vers la personne ${ }^{7}$. Les absences répétées du salarié protégé l'isolent du collectif de travail qui réagit à la gêne occasionnée par le salarié sans tenir compte du fait que celui-ci détient un mandat, ainsi que le raconte un secrétaire de comité d'entreprise.

\footnotetext{
"P. n'était pas là depuis des années, ça faisait des mois et des mois qu'il était en maladie, donc les gens ici sont très, comment le dire... C'est leur entreprise en fait, donc une personne qui nuit à leur entreprise, elle ne va pas rester dans leur cœur. Une personne qui n'est jamais là, qui est toujours absente, qui fait chier le monde parce qu'elle veut un mi-temps thérapeutique, ou des trucs comme ça, je pense qu'ils n'ont pas trop rechigné qu'on l'ait licencié." (Secrétaire du comité d'entreprise, non-syndiqué, 47 ans, PME)
}

\footnotetext{
${ }^{1}$ Nous soulignons.

${ }^{2}$ Ce constat a été mentionné lors d'entretiens réalisés avec deux médecins du travail de la région parisienne (l'un exerçant au sein de la FNATH, fédération nationale des accidentés du travail et handicapés dans le cadre d'une consultation "souffrance au travail") mais aussi lors d'interventions de deux médecins du travail au sein de l'ISST.

${ }^{3}$ Cezard M., Hamon-Cholet S., (1999), "Travail et charge mentale", Premières synthèses 99.07, n 27.1

${ }^{4}$ Fondation Européenne pour l'Amélioration des Conditions de Vie et de Travail, "La deuxième enquête européenne sur les conditions de travail", 1997, Office des publications officielles des Communautés européennes.

${ }^{5}$ Cf. Nicole Maggi-Germain, «Harcèlement moral et conditions de travail», Bulletin social Francis Lefebvre $\mathrm{n}^{\circ}$ 8/9, 2002 , p.

470 à 472 ; "Le stress au travail", RJS 3/2003, p. 191 à 200.

${ }^{6}$ Nous revenons plus loin sur ces questions.

${ }^{7}$ Infra, IIe partie.
} 
D'autre part, ce détournement du droit pose aussi la question du rôle et de la place des médecins du travail qui "cautionnent" de telles pratiques qui, selon les termes d'un médecin du travail, rendent service mais constituent, "du point de vue du métier du médecin du travail, un arbitraire terrible". Par-delà la question du statut du médecin du travail, qui apparaît extrêmement ambigu ${ }^{1}$ et suspect pour certains salariés protégés, se pose le problème du sens de la décision médicale appelée à régler des conflits sociaux.

Signalons que l'inaptitude n'est pas uniquement une invention qui a l'intérêt de régler des sorties de crise quand la situation semble totalement bloquée. En effet 5\% des personnes exerçant ou ayant exercé une activité déclarent un problème chronique de santé ou un handicap ${ }^{2}$.

\section{C) Les transactions révélatrices de l'existence d'un départ négocié}

L'analyse de certains entretiens montre aussi que l'usage fait des transactions, qui obéissent à un cadre juridique précis (1) aboutissent parfois à un contournement de la règle de droit (2)

\section{1) Le cadre social et juridique du recours à la transaction}

Sur l'ensemble des dossiers examinés, trois salariés protégés et un ancien secrétaire de comité d'entreprise ont signé une transaction avec leur employeur, autrement dit "signent un contrat par lequel les parties mettent fin à une contestation en s'accordant des concessions réciproques" ${ }^{\prime 3}$.

Issue du Code civil, la transaction constitue un mécanisme juridique destiné à régler les conséquences juridiques et financières de la rupture du contrat de travail.

\begin{tabular}{|} 
EXTRAITS DU TITRE QUINZIEME DU LIVRE TROISIEME DU CODE \\
CIVIL : DES TRANSACTIONS \\
Article 2044 \\
"La transaction est un contrat par lequel les parties terminent une contestation née, ou préviennent une contestation \\
à naître. Ce contrat doit être rédigé par écrit" \\
Article 2048 \\
"Les transactions se renferment dans leur objet : la renonciation qui y est faite à tous droits, actions et prétentions, \\
ne s'entend que de ce qui est relatif au différend qui y a donné lieu." \\
Article 2052 \\
"Les transactions ont, entre les parties, l'autorité de la chose jugée en dernier ressort. Elles ne peuvent être \\
attaquées pour cause d'erreur de droit, ni pour cause de lésion" \\
Article 2053
\end{tabular}

\footnotetext{
${ }^{1}$ Cf. Colette Jacques, "Travail et santé : le point de vue du médecin", Dr. Soc. mai 2002, p. 479 à 484.

${ }^{2}$ Waltisperger D. (2004), "Le travail est rendu responsable d'un problème de santé sur cinq", Premières informations, mai $2004, n^{\circ} 19.1$

${ }^{3}$ Cass. Soc. 13 oct. $1998, n^{\circ} 85-45309$.
} 
"Néanmoins une transaction peut être rescindée, lorsqu'il y a erreur dans la personne ou sur l'objet de la contestation. Elle peut l'être dans tous les cas où il y a dol ou violence"

Article 2058

"L’erreur de calcul dans une transaction doit être réparée»

Le recours à une transaction peut avoir différentes significations pour les salariés protégés. Dans tous les cas de figure, elle leur permet de recevoir une somme d'argent généralement largement supérieure aux six mois de salaires dus lorsque le licenciement est reconnu sans cause réelle et sérieuse. Pour ceux qui en bénéficient, cela peut être une manne à laquelle il apparaît difficile de résister.

"Mais, à ce moment-là, vous êtes dans des pressions et des propositions, vous ne savez plus où
vous nagez, on vous fait miroiter de l'argent. En plus, [l'inspecteur du travail] était monté en disant
: attention Monsieur [le Directeur Général], il y a un cas P., je n'en veux pas deux. Vous ne savez
plus où vous en êtes à la fin. Sérieusement, j'ai été assez déstabilisé par les faits, ensuite par les
procédures, parce que je n'étais pas trop préparé à ce genre de choses. Vous savez, là-dedans, les
gagnants, ce n'était pas moi, ce sont les avocats." (Salarié protégé, délégué syndical, membre du
comité d'entreprise, 55 ans, cadre, BEPC, PME, demande de licenciement pour inaptitude)

Dans le cadre d'un conflit interpersonnel fort, la transaction est vécue comme le prix à payer pour une séparation et pour que "l'employeur ait la paix".

"(Pourquoi ils ont accepté de négocier, pourquoi ils ne vous ont pas licencié pour faute grave ?)
Quand j'ai vu le directeur lors de cet entretien, il m'a dit que j'étais une grosse source de désordre
dans l'entreprise et ensuite il m'a dit, pour mémoire, il y a déjà eu une source de désordre dans
l'entreprise, c'était O. L., alors il y a eu un grand blanc. Pour moi, c'était très clair car ce monsieur,
c'était le chef d'exploitation quand ce directeur est arrivé, et à la fin de la 1 ère saison, il l'a licencié
pour faute grave. Donc pour moi c'était simple, soit tu te barres, soit je te fais le même
plan." (Salarié protégé, membre comité d'entreprise, délégué du personnel, non-syndiqué, 29 ans,
cadre, Bac+2, PME, demande de licenciement économique)

C'est également cette idée que l'on retrouve exprimée par un directeur de société ayant eu recours à une transaction.

"Ça c'est fini comme ça, il n'y a pas eu d'intervention des délégués, il n’y a pas eu de prud'hommes, il n'y a rien eu du tout. Il y a eu un accord transactionnel, c'est lui qui a fixé les sommes, mon président a été d'accord." (Directeur établissement, PME)

Lorsque la séparation n'est pas le fruit d'un conflit interpersonnel, le salarié protégé interprète le recours à la transaction comme une reconnaissance du travail accompli.

"Je pense qu'ils ont vu que je n'étais pas quelqu'un qui cherchait, comme un syndicat qui cherche vraiment à rentrer en conflit. Je pense qu'ils ont vu que j'étais une personne sensée et qui essayait de faire les choses au mieux, même si c'était terrible pour tout le monde. Et pour aller dans ce sens, pour me remercier peut-être de la façon dont ça c'est passé, je pense que c'est ça qui a fait qu'ils acceptent. Enfin, ils sont revenus sur ce que j'ai demandé bien sûr, mais ils ont accepté la transaction financière pour le principe." (Salariée protégée, déléguée du personnel, non-syndiquée, 55 ans, profession intermédiaire, sans diplôme, TPE, demande de licenciement économique)

La transaction est aussi présentée comme le moyen de quitter l'entreprise sans démissionner et perdre les droits au chômage. 
"[Si] on est licencié, on bénéficie du chômage donc c'est plus facile de partir. Si on démissionne, on n'a rien. Moi dans mon cas, je n'en pouvais plus, l'ambiance au niveau du service n'était pas terrible." (Salariée protégée, délégué du personnel, membre du CHSCT, non-syndiquée, 33 ans, CAP, agent de maîtrise, PME, demande de licenciement économique)

Le mandat apparaît, pour le salarié protégé, comme une ressource qu'il peut éventuellement faire valoir dans les négociations.

\begin{abstract}
"(Sauf que vous êtes protégé quand même, c'est intervenu, ça dans la négociation ?) Pas directement. Moi, je gardais ça sous le coude, évidemment, je le savais, enfin je ne suis pas aller les voir en disant : je suis protégé, j'ai telle et telle chose, vous ne pouvez pas me virer. Je pense qu'ils l'avaient intégré dans la négociation, moi quand j'ai fait la proposition, on s'est vu un jour en début d'après-midi, le soir quand on s'est vu à 5 heures, il m'a dit c'est $\mathrm{OK}$, on accepte. Je pense que j'avais fait une proposition cohérente." (Salarié protégé, membre comité d'entreprise, délégué du personnel, non-syndiqué, 29 ans, cadre, Bac+2, PME, demande de licenciement économique)
\end{abstract}

Le jugement des secrétaires de comité d'entreprise apparaît plus tranché; ils se montrent critiques à l'égard des transactions, faisant entrer la dimension collective dans leur jugement. Cette critique n'est pas étonnante venant de salariés occupant des fonctions plus politiques où sont surtout élus des militants syndicaux. De ce fait, ils sont plus que les autres sur des logiques de positions et condamnent tout ce qui peut apparaître comme une posture individuelle.

C'est le cas du témoignage d'un secrétaire de comité d'entreprise, lorsqu'il compare les départs volontaires signés dans le cadre d'un plan de sauvegarde de l'emploi et les transactions.

"Il n'y a pas eu de transactions ou de magouilles [lors des départs volontaires]. Elle a bénéficié de 10 milles euros mais au même titre que les autres qui acceptaient le volontariat. (Pourquoi vous parlez de magouille ?) Il y aurait pu avoir une transaction. (Et la transaction pour vous c'est une magouille ?) Ça peut l'être si ce n'est pas transparent. [...] Parce qu'il y a des gens qui pourraient profiter de leur mandat pour se vendre. Mais là je n'ai pas de preuves, je n'ai que des rumeurs et comme je n'aime pas trop les exploiter je n'irais pas plus loin dans ce débat".» (Salarié protégé, délégué syndical, membre CHSCT, syndiqué, ouvrier, 44 ans, CAP, demande de licenciement pour faute)

C'est également ce qu'exprime un autre secrétaire de comité d'entreprise.

\begin{abstract}
"Moi je ne trouve pas ça normal, mais s'il a voulu, c'est son problème, s'il a été d'accord, nous on n'aurait pas été d'accord. Je sais que quand l'ancien comptable est parti, il y a eu une transaction aussi, ils se sont arrangés entre eux. Je trouve que ce n'est jamais bon de s'arranger entre 5 témoins. (Pourquoi, après tout ça règle le problème facilement, ça ne fait pas de bruit.) Oui, c'est ça, ça ne fait pas de bruit, c'est un cadre, je ne suis qu'un simple ouvrier. (C'est des cas spéciaux ?) Non, je ne dis pas ça, je trouve ça un peu dommage. Généralement quand il y a une transaction, c'est qu'il y a 2 parties, qu'une partie qui veut appuyer sur le fait qu'elle a des informations que l'autre n'aimerait pas qu'on connaisse, ça se monnaie peut-être, enfin je ne sais pas, c'est une hypothèse. (Pour vous c'est l'intérêt.) Oui, c'est qu'il y a de l'intérêt, qu'il y a un témoin, des choses qu'on n'a pas envie que les gens entendent. " (Secrétaire comité d'entreprise, syndiqué, 58 ans, PME)
\end{abstract}

Le reproche qui semble être fait est que le salarié protégé utilise soit son mandat, soit des informations obtenues dans le cadre du mandat à des fins personnelles, et ce dans une certaine opacité. Quand les salariés de l'entreprise finissent par l'apprendre et par en connaître les termes, cela pose problème. 
"(Et comment c'est perçu par les autres salariés ?) Très mal, là vous pouvez y aller, vous pouvez descendre interroger qui vous voulez dans l'atelier, très mal. (On dit) qu'il est parti avec le pactole, à 56 ans, c'est pas mal. Moi à 56 ans, on me donne ce qu'ils lui ont donné, ça va, je m'en vais, et je ne demande même pas les ASSEDIC, et tous les gens pensent ça, c'est très clair." (Secrétaire du comité d'entreprise, non-syndiqué, 47 ans,PME)

Il est vrai qu'une telle utilisation de la transaction apparaît comme un contournement de la règle de droit.

\section{2) Le contournement de la règle de droit}

La transaction constitue, dans trois des quatre cas analysés, une manifestation d'un départ qui a été, en pratique, négocié, même si l'employeur a formellement recours, pour rompre le contrat de travail, au licenciement. Autrement dit, la qualification juridique de la rupture, c'est-à-dire le licenciement, ne sert que d'habillage à une décision commune. La proposition de transaction, qu'elle soit le fait de l'employeur ou du salarié, constitue alors une indication, pour l'autre partie, d'une impérieuse nécessité de mettre fin à la relation de travail. Le contexte de ces transactions est particulièrement conflictuel. Départ négocié et transaction se confondent parfois, ainsi que l'exprime un directeur général.

"Il n'y avait qu'une solution, c'était d'arriver à un accord à l'amiable, sinon on allait vraiment pas s'en sortir, lui parce qu'il voulait du fric pour atteindre son âge de préretraite où je ne sais plus trop quoi, c'était ça son argument. Nous, parce qu'il fallait bien qu'on s'en sorte du problème, on n'arrivait plus à gérer la boîte dans le domaine technique. Allez demander aux salariés..." (Directeur général, PME)

Or il est de jurisprudence constante que seuls la démission et le licenciement peuvent juridiquement mettre un terme au contrat de travail d'un salarié protégé. Il n'existe pas d'autre cause juridique valable. Selon la chambre criminelle de la Cour de cassation, proposer à un représentant du personnel de mettre fin au contrat de travail par un accord de résiliation amiable constitue un délit d'entrave, pénalement répréhensible.

Cass. crim., 6 janv. 2004 ; F. M.-C. et A : Juris-data n²004-021966.
Extraits
"Les dispositions législatives soumettant à l'assentiment préalable du comité d'entreprise ou à la décision
conforme de l'inspecteur du Travail le licenciement des salariés légalement investis de fonctions
représentatives, ont institué au profit de tels salariés et dans l'intérêt de l'ensemble des travailleurs qu'ils
représentent, une protection exceptionnelle exorbitante du droit commun qui interdit, par suite, à
l'employeur de poursuivre par d'autres moyens la résiliation du contrat de travail' $;$ l'employeur ne saurait
se soustraire à l'observation de ces dispositions d'ordre public en proposant au salarié de mettre fin au
contrat de travail par un accord de résiliation. Dès lors, c'est à tort que pour confirmer la décision de relaxe
des prévenus poursuivis pour entrave à l'exercice du droit syndical, pour avoir, après un refus d'autorisation
de licenciement opposé à l'employeur par l'inspecteur du travail, proposé à la déléguée syndicale un accord
de résiliation de son contrat de travail moyennant versement d'une somme égale à dix huit mois de salaire,
la cour d'appel énonce "qu'une telle offre n'a en soi rien de discriminatoire, qu'elle est même plutôt
généreuse au regard des usages, qu'il est compréhensible qu'un employeur cherche à rompre un contrat avec
une personne avec qui il est en relations conflictuelles, et ce, indépendamment de toute appartenance
syndicale de l'intéressée" ; en effet, en prononçant ainsi, les juges, qui ont refusé de tirer les conséquences
légales de leurs propres constatations, ont violé les articles L. 412-18 et L. 481-2 du Code du travail ainsi
que le principe ci-dessus rappelé.

\footnotetext{
${ }^{1}$ Nous soulignons.
} 
La cour de cassation ne s'arrête d'ailleurs pas à la qualification juridique donnée par les parties : elle a ainsi pu décider que l'acte qualifié de transaction qui a en réalité pour objet de résilier le contrat de travail, était nul ${ }^{1}$. Le raisonnement suivi par la juridiction suprême est clair et possède sa propre cohérence au regard des dispositions du Code du travail : la rupture d'un commun accord est nulle parce qu'elle conduit à écarter les dispositions légales protectrices. C'est parce que le Code du travail a institué une protection exorbitante "dans l'intérêt de l'ensemble des travailleurs" que la rupture amiable ou d'un commun accord est impossible, à peine de nullité. Autrement dit, ce n'est pas tant le salarié que le mandat, en tant que fondement de l'institution, que le juge cherche à protéger. Cette interprétation apparaît d'ailleurs conforme aux textes : l'article L. 425-1, $8^{\mathrm{e}}$ alinéa du Code du travail, qui étend, pendant une durée de six mois, la protection des salariés protégés aux "salariés qui ont demandé à l'employeur d'organiser les élections de délégués du personnel, ou d'accepter d'organiser ces élections" et se réfère, dans la justification qu'il apporte, au fait de "faciliter la mise en place de l'institution ${ }^{2}$ des délégués". C'est ce qui explique que la Cour de cassation, dans un arrêt publié au Rapport (c'est-à-dire particulièrement important du point de vue de la juridiction elle-même ${ }^{3}$ ) sanctionne par la nullité absolue (et non relative) "toute transaction conclue entre l'employeur et le salarié avant la notification de son licenciement prononcé après autorisation de l'autorité administrative", faisant ressortir, par la manière dont est rédigé l'arrêt, le lien entre intérêt des salariés et nullité de la transaction.

Cass. Soc. 10 juillet 2002, Costes c/ Sté Innotech international, ${ }^{\circ} \mathbf{2 5 0 0}$ FS-PBRI
"Mais attendu que la protection exceptionnelle et exorbitante du droit commun des salariés investis de
fonctions représentatives a été instituée, non dans le seul intérêt de ces derniers, mais dans celui de
l'ensemble des salariés; qu'il en résulte qu'est atteinte d'une nullité absolue d'ordre publique toute
transaction conclue entre l'employeur et le salarié avant la notification de son licenciement prononcé après
autorisation de l'autorité administrative"

Il est intéressant de rappeler que si la nullité, sanction prononcée par le juge, entraîne la disparition rétroactive de l'acte, la nullité est absolue "lorsque les conditions imposées par la loi sont essentielles et tendent à protéger l'intérêt général, ou l'ordre public, ou les bonnes mœurs" ${ }^{4}$. C'est donc bien, dans les textes et dans la jurisprudence de la Cour de cassation, l'intérêt collectif, voire l'intérêt général ${ }^{5}$ qui prime sur l'intérêt de la personne. Or, ces deux "dimensions", qui apparaissent en constante tension, à la fois dans les

\footnotetext{
${ }^{1}$ Cass. soc. 3 avril $2001-\mathrm{N}^{\circ} 118$.

${ }^{2}$ Nous soulignons.

${ }^{3}$ La Cour de cassation fait référence, dans les arrêts qu'elle rend, à des lettres qui constituent une codification informant le lecteur de la place qu'elle accorde à l'arrêt ainsi rendu. P pour publié dans le Bulletins des arrêts de la Cour de cassation, B pour publié au flash adressé aux magistrats, I pour une diffusion internet via le site de la Cour, R pour publié au rapport ce qui "manifeste la force doctrinale évidente puisque dès son prononcé, la Cour de cassation indique qu'il sera commenté par elle-même dans le rapport annuel qui sera édité l'année suivante" (T. Grumbach, "doctrine et déontologie", Dr. Soc. avril 1999 , p. 323 à 325 , p. 324.).

4 "La nullité est dite relative lorsqu'elle sanctionne une règle destinée à protéger une partie de l'acte", R. Guillien et J. Vincent (dir.), Lexique des termes juridiques, Dalloz, $13^{\mathrm{e}}$ éd., 2001.

${ }^{5}$ La justification de l'intérêt général est fréquemment admise : c'est ainsi que l'inspecteur du travail peut refuser d'autoriser le licenciement pour des motifs d'intérêt général qui peuvent consister

- à maintenir une représentation du personnel dans l'entreprise (CE 22 juin 1987, n 74627),

- ou encore prévenir une augmentation des tensions sociales dans et hors de l'entreprise (CE 21 décembre 1994, $n^{\circ}$ 149089).

Suivant la circulaire DRT de 2000, "Il est rappelé que l'intérêt général n'a lieu d'être invoqué que lorsque le refus d'autorisation est nécessaire pour rétablir ou maintenir la paix sociale dans l'entreprise ou bien lorsque l'autorisation de licenciement entraînerait la disparition de l'institution représentative du personnel concernée" Circulaire DRT n ${ }^{\circ} 3$ du 1er mars 2000, Ministère de l'emploi et de la solidarité, Dr. Ouvrier oct. 2000, p. 442 à 451, p. 444.

Mais ni la situation personnelle du salarié protégé (CE 29 juillet 1993, Sté Bpda, n 32.197), ni même la volonté de maintenir une organisation syndicale représentée dans l'entreprise (CE 8 nov. 1993, Les mutuelles de Bretagne, ${ }^{\circ}$ 120.034) ne peuvent fonder l'existence d'un intérêt général.
} 
pratiques mais également dans les textes, se nourrissent de l'ambivalence du droit qui situe le salarié protégé sous contrat et sous statut ${ }^{1}$ et implique d'articuler règles du droit commun et statut exorbitant dérogatoire lequel trouve sa justification en même temps que son fondement dans les finalités du mandat: l'existence de l'institution créée dans l'intérêt des travailleurs. Et finalement, en "achetant le départ et l'absence de procès", le recours à la transaction comme contournement de l'interdiction légale de recourir au départ négocié met en porte-àfaux les textes et la pratique, déplaçant la problématique de l'occupation du mandat sur un terrain purement individuel. D'autre part, en tant que procédure juridique, la transaction pose la question de l'égalité des salariés protégés, la possibilité d'y recourir ainsi que le montant versé par l'employeur semblant largement dépendre de la "capacité de nuisance" du salarié et des ressources dont ils disposent (recours à un avocat comme "guide juridique", capacité à mobiliser d'autres salariés afin d'étayer le dossier...).

Dans un tel contexte, l'inspecteur du travail peut parfois apparaître comme une simple chambre d'enregistrement. Car même si, comme le rappelle la Cour d'appel de Paris, le constat de l'existence d'une transaction n'est pas de nature par elle-même à justifier l'autorisation de licenciement d'un salarié protégé ${ }^{2}$, l'existence d'une transaction s'impose en pratique à l'inspecteur du travail qui ne fait alors qu'avaliser une décision déjà prise.

Cette absence de prise sur un litige où tout est déjà joué se retrouve dans les décisions d'inspecteurs du travail.

Inspection du travail
XXX section
Affaire suivie par XXX.
Objet: décision de licenciement
L'inspecteur du travail de la XXX section,
Vu la demande d'autorisation de licenciement pour motif d'inaptitude au poste de travail, présentée le xxx
2002 par l'entreprise Z. et concernant Monsieur P., élu du comité d'entreprise, délégué du personnel,
membre du CHSCT et délégué syndical CGC;
Vu les articles L $425-1, \mathrm{~L} 4361, \mathrm{R} 436-1$ et suivants du code du travail ;
Vu la procédure d'entretien préalable qui a eu lieu le xxx $2002 ;$
Vu l'avis du comité d'entreprise en date du xxx $2002 ;$
Vu la prolongation du délai d'enquête notifiée le xxx $2002 ;$
Vu les éléments recueillis lors de l'enquête contradictoire du xxx 2002 ;
Considérant la réalité du motif d'inaptitude médicale;
Considérant néanmoins que l'enquête a permis de constater que la survenance de cette inaptitude
s'explique essentiellement par un litige ancien et non réglé sur le contrat de travail et le poste de Monsieur $_{P .3^{3}}$

\footnotetext{
${ }^{1}$ Supra. Ce constat était déjà celui que dressait l'avocat général Touffait dans ses conclusions rendues dans l'affaire "Perrier" qui ont posé pour la première fois en principe l'interdiction de la rupture amiable pour les salariés protégés : "[...] il existe une incompatibilité certaine entre les mécanismes issus de la théorie contractuelle et le statut protecteur légal des représentants du personnel", Touffait, Cass. Chambre mixte 21 juin 1974, Perrier, Dalloz 1974, p. 593 à 604, p. 601.

${ }^{2}$ CAA de Paris, $3^{\text {e }}$ chambre, n ${ }^{\circ 7 P A 01962 ~ d u ~} 22$ septembre 1998, citée par R. Mélot, op. cit., p. 347.

${ }^{3}$ Nous soulignons.
} 


\begin{abstract}
Considérant que l'employeur a alors modifié unilatéralement le contrat de Monsieur P. restreignant ses responsabilités dans une ambiance à la tension croissante, proche de la définition légale du harcèlement moral au travail, alors même que du fait de ses mandats aucune modification unilatérale ne pouvait être imposée à Monsieur P.
\end{abstract}

Considérant que ces faits permettraient de motiver une décision de refus d'autorisation de licenciement ;

Considérant cependant qu'au cours de l'enquête Monsieur P. a confirmé qu'il estimait désormais impossible le retour à une situation de travail normale et qu'il souhaitait un licenciement pour inaptitude assorti dune transaction mettant fin aux procédures civiles engagées

Considérant l'impossibilité «assurer le reclassement de Monsieur P. dans l'entreprise,

DECIDE

L'autorisation de licenciement de Monsieur P. est accordée.

Fait à X, le xxx 2002

L'inspecteur du travail de la XXX section

$\underline{\mathrm{XXX}}$.

La volonté de partir émise par le salarié protégé conduit l'inspecteur du travail à avaliser une procédure pourtant irrégulière au plan de l'analyse juridique. Certains adoptent, dans la rédaction de leurs décisions ${ }^{1}$, de véritables stratégies cherchant à rendre compte de la nécessaire conciliation, voire de véritable compromis, ainsi que s'en explique un inspecteur du travail.
"Je trouve important de montrer à l'interlocuteur qu'on n'est pas dupe du caractère irrégulier, c'est pour ça que je mets à la fin : décide néanmoins. C'est-à-dire paradoxalement d'accorder, compte- tenu de la volonté affirmée du salarié, et des fois on leur fait même signer des papiers comme quoi c'est eux qui demandent. Et en droit c'est un problème parce qu'on ne doit pas tenir compte, et c'est assez logique, de la volonté du salarié, parce que c'est une institution derrière la personne qui est protégée. Est ce qu'on la protège vraiment ? C'est vrai que je n'en suis plus sûr." (Inspecteur du travail)

Toutefois, la question de l'autorisation de l'inspecteur du travail peut se poser. Les inspecteurs du travail jugent, en premier lieu, la moralité de l'accord. Les critères retenus sont très divers. Certains portent un regard sévère sur les mandats "d'opportunité" et ont donc tendance à refuser tout accord, si le salarié n'a pas eu l'investissement qu'implique un mandat de représentant des salariés.

"Ça m'est arrivé d'adopter cette position, et ça m'est arrivé vis-à-vis de salariés, dont je pouvais savoir pour avoir une connaissance de l'entreprise et de leur activité, qu'en fait, ils n'avaient aucune activité, et que c'était un mandat de pure protection, et qu'à la fin, ils le monnayaient pour partir avec de meilleures conditions que des salariés qui n'avaient pas de mandat." (Inspecteur du travail)

"Je demande systématiquement les comptes-rendus des réunions de CHSCT. Donc on voit bien qui intervient, qui n'intervient pas [...], avec quelle qualité d'intervention, quelle pugnacité aussi des fois. Et vous voyez bien aussi quels sont ceux qui viennent dans votre bureau et vous disent là il faut agir. [...] Vous allez vous-même aux réunions du CHSCT quand vous pouvez y aller, donc à ce moment-là, vous voyez bien aussi qui intervient, qui met en œuvre, qui s'oppose, qui prend des risques, etc..." (Inspecteur du travail)

\footnotetext{
${ }^{1} \mathrm{Cf}$. Iie partie, les développements sur la fonction de restitution juridique de l'inspecteur du travail.
} 
Le jugement moral ne porte pas uniquement sur le comportement du salarié mais aussi et principalement sur les conséquences que ce comportement peut avoir sur le système de représentation dans son ensemble.

\begin{abstract}
"Je protège aussi, je pense, l'institution. Je pense que les rumeurs courent vite dans les entreprises, et un représentant du personnel, aussi bon qu'il ait pu l'être d'ailleurs en tant que représentant du personnel, lorsqu'il part avec sa transaction, il y aura toujours des salariés pour dire : lui a droit à une transaction mais pas nous. [...] Pour le salarié qui n'a joué aucun rôle et qui n'a fait que se planquer en prenant ce mandat et qu'il puisse partir en le monnayant, là effectivement, je pense que c'est une décrédibilisation totale du mandat vis-à-vis des salariés." (Inspecteur du travail)
\end{abstract}

Toutefois, les appréciations que peuvent porter certains inspecteurs du travail sur le comportement des salariés ne sont pas uniquement des leçons de morale mais sont fortement pondérés par d'autres considérations.

"Et là, effectivement, je me mets à leur place en me disant, moi j'ai la sécurité de l'emploi, c'est très facile de dire à quelqu'un vous resterez dans l'entreprise, et bannière en avant, et droit du travail, etc..." (Inspecteur du travail)

Ceci explique les hésitations ou les décisions différentes que peuvent prendre les inspecteurs du travail pour des cas, a priori sensiblement identiques. On retrouve, sur ce point, l'opposition classique que Weber (1959) formule à propos des décisions et des hommes politiques : éthique de conviction et éthique de responsabilité. Certains ne considèrent que la fonction et accordent la plus grande importance au maintien d'une représentation du personnel. Pour eux la fonction, parce qu'elle échappe à l'individu qui n'est que le dépositaire de l'intérêt général, ne saurait faire l'objet d'arrangements. D'autres au contraire font intervenir dans leur décision la situation plus ou mois difficile de l'individu qui occupe la fonction.

En outre, l'inspecteur du travail tente de faire la différence dans les situations qu'on lui présente, pour faire la part entre les accords "spontanés" et les accords forcés. Certains inspecteurs du travail font clairement pression pour qu'un accord plus équilibré soit conclu, tentant ainsi de contrebalancer l'inégalité entre dirigeants et salariés.

\footnotetext{
"Quand le salarié et l'employeur n'arrivent pas à se mettre d'accord sur le principe ou le montant de la transaction, il m'est arrivé dans certains cas de refuser une autorisation de licenciement dans un $1^{\text {er }}$ temps, en me basant sur les éléments juridiques que j'avais pour refuser, et puis le fait qu'il y ait un refus, que l'employeur soit du coup devant une impasse, c'est l'élément déclencheur de la négociation et ils reviennent me voir ensuite en disant : "ça y est, on a négocié une transaction, est ce que vous pouvez, soit sur le mode d'un recours gracieux, soit sur le mode d'une nouvelle demande, mais est-ce que vous pouvez, en fonction de cet élément nouveau, me faire l'autorisation de licenciement." (Inspecteur du travail)
}

Il est intéressant de constater que si les inspecteurs du travail respectent la volonté du salarié protégé de quitter l'entreprise, certains mettent ainsi en place de véritables stratégies d'aide aux salariés protégés, comme l'indique la citation précédente.

Si l'inspecteur du travail peut interférer sur le déroulement de la transaction, il ne pèse guère dans la décision rendue dès lors que les parties ont préalablement décidé, d'un commun accord, de mettre un terme à la relation de travail et de recourir à une transaction. Face à un nombre de transactions en augmentation, selon le constat dressé par les inspecteurs du travail, on ne peut que s'interroger sur l'effectivité des dispositions légales protectrices du statut et sur le rôle de l'inspecteur du travail ${ }^{1}$. La transaction vide, de par ses finalités, la procédure de

\footnotetext{
${ }^{1}$ Deux cas de figures peuvent se présenter : le salarié fait ouvertement état devant l'inspecteur du travail de la transaction ou il la cache. Mais face à l'absence de sérieux, l'inspecteur du travail "pressent" généralement l'existence de la transaction.
} 
contrôle et de validation de son sens. Un inspecteur du travail y voit une "façon de monnayer le mandat". 


\section{Conclusions intermédiaires}

La confrontation des textes juridiques et des pratiques montre l'existence d'un certain décalage qui conduit à poser la question de l'effectivité de la règle de droit et de son application. Une application dévoyée du droit, qui se traduit par un contournement ou une distorsion de la règle, conduit nécessairement à s'interroger sur la pertinence du maintien en l'état des règles. Se pose alors la question du passage d'une effectivité relative, acceptable voire souhaitable dans une société démocratique, à une ineffectivité qui imposerait une transformation de la norme juridique. Le problème qui risque en effet de se poser est celui de l'effet pervers de la règle : c'est le cas s'agissant de l'utilisation de l'inaptitude ou encore de la transaction qui "facilitent" la sortie des salariés protégés de l'emploi, allant à l'encontre des principes juridiques qui sous-tendent la mise en place d'instances représentatives du personnel mais provoquant aussi des effets dévastateurs sur les autres salariés demeurés dans l'entreprise ou licenciés moyennant le versement des indemnités légales.

D'autre part, ce décalage ne peut que nous amener à nous interroger sur le sens de ces évolutions : mal-être au travail mais aussi engagements dans des mandats représentatifs qui s'inscrivent aussi dans des parcours personnels. C'est peut-être cet équilibre qui est parfois remis en cause : la transaction évite au salarié de démissionner et lui permet de se retirer d'une situation de travail devenue parfois insupportable tout en pouvant espérer toucher les indemnités de chômage. La dimension symbolique du droit s'efface devant une acception instrumentale. On ne peut dès lors qu'approuver le récent revirement de jurisprudence de la Cour de cassation qui ouvre au salarié dit «protégé » la possibilité de demander au juge la résiliation de son contrat de travail. Prononcée aux torts de l'employeur, elle produira les effets d'un licenciement sans cause réelle et sérieuse : l'initiative de la rupture incombe au salarié mais elle est imputable à l'employeur qui devra en supporter les conséquences financières.

Mais c'est parce que le salarié dit «protégé » se trouve dans des liens contractuels que la Cour de cassation lui ouvre, depuis un récent arrêt très commenté et « estampillé » du sceau des arrêts particulièrement importants ${ }^{1}$,

Peut-être aussi faut-il voir dans ces évolutions l'extension d'un modèle de marchandisation des relations de travail. C'est ce qu'explique un responsable des relations sociales au sein d'un grand groupe.

"Mais ce plan social a fait une brèche dans l'esprit de [l'entreprise], parce qu'après, ça a introduit
une culture où l'on pouvait négocier son départ [...]. Sur le dernier plan social, on a fait un départ
sur 4 , c'est-à-dire que $25 \%$ de la population est partie, ce qui est énorme, et nous on pensait
vraiment qu'on aurait pas assez de volontaires." (DRH, grande entreprise)

Toutefois, on peut aussi regarder les usages décalés du droit d'une autre façon. Il faut comprendre que l'usage strict des seules règles juridiques peut conduire à des situations invivables pour l'une ou les deux parties. Le compromis officialisé par l'accord, même s'il déroge aux règles peut être considéré comme "une des grandes inventions de l'humanité" pour

\footnotetext{
${ }^{1}$ La Cour de cassation « classe » ses décisions suivant une certaine nomenclature : P (publié au Bulletin des arrêts de la Cour de cassation (édition mensuelle), B (publié, en résumé, au Bulletin d'information de la Cour (bimensuel), R (rapport annuel), I (site internet), NP (non publié).
} 
reprendre Simmel (2003), car il permet des règlements qui n'exigent pas la destruction de l'adversaire.

L'inaptitude ou la transaction ne sont pas toujours ni recherchées ni acceptées avec emphase par les salariés. Elles sont aussi un pis-aller, qui permet de mettre à distance, au moins pour un temps, des situations que l'on a le plus grand mal à supporter.

Les effets d'un conflit de longue durée sont loin d'être anodins pour les individus, surtout qu'ils se vivent souvent sur la base d'une inégalité de ressources dont les effets sont dévastateurs sur leur personnalité.

C'est en grande partie pour ces raisons que des inspecteurs du travail peuvent autoriser les licenciements "convenus" car ils font preuve d'empathie par rapport au sort des salariés. En effet, la principale raison pour laquelle les salariés acceptent des transactions ou demandent leur licenciement pour inaptitude, c'est parce que le licenciement permet de sortir d'une situation de crise, parfois totalement insoutenable pour le salarié, tout en gardant le bénéfice des allocations chômage. Il est probable que si la démission d'un salarié protégé lui offrait les mêmes garanties, le nombre de licenciements subirait une baisse au profit des démissions qui, elles ne dépendent pas de la décision de l'inspecteur du travail. 


\section{CONCLUSION GENERALE}

La question qui avait été posée en préalable à cette enquête portait à la fois sur les causes de la stabilité des demandes de licenciement des salariés protégés et sur leur plus grande acceptation. À l'issue de l'étude, plusieurs réponses peuvent être avancées.

Une première explication peut être tirée de la présence de contraintes exogènes qui s'imposent indifféremment le salarié protégé et son employeur : c'est le cas pour certaines inaptitudes et pour une grande partie, des licenciements pour motifs économiques. Ces derniers, dès lors qu'ils s'accompagnent de plans de sauvegarde de l'emploi comportant des dispositions qui peuvent apparaître, d'un certain point de vue, relativement avantageuses, et intégrant un plan de volontariat peuvent expliquer que certains salariés protégés puissent se prononcer pour leur intégration dans le volant de ceux qui seront touchés par les licenciements.. D'autre part, certains licenciements économiques collectifs ne sont pas perçus par les salariés comme des licenciements mais comme des mesures d'âge permettant aux salariés concernés de pouvoir enfin se retirer de l'activité professionnelle.

Toutefois, cette acceptation "consensuelle" est loin d'épuiser l'explication, car tous les licenciements ne sont pas acceptés par les salariés protégés.

En premier, les motifs de licenciement ne peuvent, à eux seuls, parvenir à expliquer les réactions des salariés. L'assentiment à un licenciement pour motif économique n'est donné par les salariés protégés que si les mesures économiques prises, qui intègrent le licenciement, apparaissent comme une fatalité. Autant que les déterminants économiques, c'est en effet la perception, par les salariés protégés, que l'entreprise ne fait pas un usage stratégique de l'évolution du contexte, qu'elle est bien soumise à des contraintes qui lui échappent, qui conditionne leur accord. En outre, le motif économique est sur représenté dans les demandes de licenciements de salariés protégés. Certains licenciements pour inaptitude se révèlent être aussi des habillages juridiques permettant de sortir de situations conflictuelles complètement bloquées.

En second, il reste un fort taux de licenciements pour lesquels l'accord ne va pas de soi au départ.

La compréhension du fondement des désaccords et de leur dynamique va donc nous permettre de compléter le premier élément de réponse.

Les salariés protégés occupent des mandats très différents, notamment quant aux enjeux qu'ils véhiculent. Ils bénéficient pour cela d'une protection qualifiée par les juges d'exceptionnelle et exorbitante du droit du commun. La jurisprudence fonde, en droit, une objectivation du mandat : il s'agit moins de protéger la personne que les fonctions qu'elle assume. C'est donc le mandat, comme lieu d'expression collective des salariés qui est l'objet de la protection. C'est aussi le mandat qui donne au salarié qui le détient un certain nombre de prérogatives, notamment de demander des comptes à l'employeur sur sa gestion, mais aussi des pouvoirs. Ces droits ainsi reconnus impliquent, corrélativement, des devoirs; leur interdépendance fondant l'idée de statut. La relation de subordination liée au contrat de travail se trouve ainsi fortement tempérée par le fait que le mandat fournit un statut qui échappe, en partie, à la relation contractuelle. 
Cette conception juridique du mandat trouve une traduction sociologique dans les comportements d'une partie des salariés protégés. Ceux-ci conçoivent leur relation à l'employeur comme antagoniste par nature, car les intérêts des salariés et des dirigeants sont différents. Porteur de l'intérêt collectif des salariés, leur mandat a pour objectif de réduire les déséquilibres de l'échange salaire - travail, qui est au désavantage des salariés. Ce sont les salariés protégés les plus militants, les plus politiques - c'est-à-dire qui se référent à une conception du monde à l'opposé des inégalités qu'ils constatent - qui décrivent cette conception du mandat. On peut alors trouver dans leur parcours un élément déterminant : le sentiment d'avoir vécu des situations injustes en raison de leur appartenance aux couches les moins favorisées de la société.

L'entreprise n'est pas toujours hostile à cette conception du rôle du représentant du personnel. Au contraire, les organisations les plus formalisées, où la gestion du personnel fait appel à des procédures et des outils standardisés, se satisfont bien de ce fonctionnement : elles y trouvent même leur compte puisqu'elles disposent d'un interlocuteur.

La congruence entre conception du mandat et formes organisationnelles configure ainsi un cadre de jeu où les salariés protégés peuvent accroître leur valeur sociale. Le droit définit en quelque sorte les règles du jeu et constitue en même temps une ressource pour objectiver les différentes positions, c'est-à-dire fixer les frontières des positions ainsi que les droits et devoirs de chacun, dotant les relations sociales d'une grande stabilité.

Par contre, dans des organisations plus petites, familiales, paternalistes, la posture oppositionnelle des salariés protégés est mal vécue par les dirigeants qui y voient une remise en cause personnelle. Le salarié protégé devient vite un gêneur dont il faut se débarrasser.

Toutefois, ces conceptions du mandat ne sont pas présentes chez tous les salariés protégés. Il apparaît intéressant, au plan de l'analyse juridique, de s'interroger sur ce que l'on peut qualifier de phénomène de banalisation du statut juridique et social du salarié protégé. De telles évolutions traduisent en même temps qu'elles l'induisent un recentrage sur la personne, recentrage accentué par la place de plus en plus importante occupée aujourd'hui par les droits fondamentaux de la la personne ${ }^{1}$, droits opposables à l'employeur en vertu de l'article L. 120$2^{2}$. On peut ainsi émettre l'hypothèse d'une tendance à la subjectivation du mandat, qui apparaît de plus en plus lié à la personne qui l'occupe plutôt qu'à la fonction de représentation détenue.

Ce glissement est encore plus perceptible au niveau sociologique. Le fait qu'un nombre important de salariés détiennent des mandats en dehors de toute référence à une organisation syndicale, quand ce ne sont pas des candidatures contre les listes présentées par les organisations syndicales, modifie la référence au collectif. Ces salariés protégés se sentent alors porteurs d'une conception de l'intérêt collectif contingente (un groupe professionnel plus que les salariés dans leur ensemble) et floue (la référence aux gens) qui orientent différemment leur action. Ils conçoivent plus que les autres leur rôle comme une aide pour les salariés, comme une capacité à représenter les intérêts conjoints de l'entreprise et des salariés.

\footnotetext{
${ }^{1}$ Ray (J.-E.), "Courrier privé et courrier personnel", Dr. Soc. 2001, p. 915 ; Favennec-Hery (F), "Vie privée dans l'entreprise et à domicile", RJS 12/01, p. 940.

2. Inséré par la loi du 31 décembre 1992: «Nul ne peut apporter aux droits des personnes et aux libertés individuelles et collectives de restrictions qui ne seraient pas justifiées par la nature de la tâche à accomplir ni proportionnées au but recherché ».
} 
Le mandat peut aussi s'inscrire dans une recherche de valorisation personnelle où l'intérêt des tâches inhérentes au mandat peut passer avant la valorisation de la représentation des salariés.

Ce mode d'occupation du mandat est alors révélateur du brouillage des repères professionnels antérieurs et d'une tertiairisation des fonctions qui s'accompagne d'une forte hétérogénéité. Il faut d'ajouter à ce constat la multiplication des statuts d'emploi et la disjonction de plus en plus importante entre le niveau de formation initiale atteint et les postes occupés. Tout cela dessine un nouveau contexte social dans lequel le rapport au travail et aux employeurs devient de plus en plus complexe, multipliant à l'infini les formes que peut prendre une carrière professionnelle et rendant l'individu moteur de cette évolution. Or, la démocratisation de l'enseignement fournit les ressources cognitives nécessaires à la maîtrise de ces nouvelles situations. De ce fait, on assiste à plus grande importance accordée à l'individu qui devient, plus que le collectif, une référence centrale, une norme de comportement. Mais cela s'accompagne, en l'absence de références collectives, d'une exposition plus importante des individus, qui deviennent de ce fait beaucoup plus fragiles.

Ce sont donc les populations les plus jeunes, les plus diplômés qui apparaissent les mieux armées pour concevoir ainsi les orientations de leur mandat. À la recherche d'une valorisation sociale qui a du mal à se concrétiser dans une évolution hiérarchique bloquée, ces salariés tentent, dans un premier temps, de reconstruire un intérêt au travail différent, ce qui passe souvent par une extension des relations professionnelles vers les champs les plus intimes de l'individu, ou dit autrement, par une importation du monde domestique dans la sphère du travail qui rend poreuse et changeante la frontière du travail et du non-travail.

Le droit, bien que protecteur du salarié protégé, apparaît, dans un tel contexte, comme un ensemble de règles exogènes, une imposition de contraintes mal adaptées aux configurations locales et un frein à l'investissement personnel. Le juridique ne devient une ressource que dans les cas de dégradation de la relation entre salariés et employeurs.

Dans une organisation très formalisée, l'investissement individuel n'est pas rejeté. Toutefois, les règles de mobilité, d'avancement ou d'augmentation de salaire se réfèrent à des indicateurs objectifs. Le retour sur l'investissement que les individus consacrent à leur activité professionnelle ne va pas de soi, car il a du mal à se traduire dans des catégories "objectives" (ancienneté et diplômes) les plus facilement intégrées dans une gestion formalisée du personnel.

Dans les entreprises plus petites où le personnel est géré beaucoup plus par des interrelations chef - salarié, cette relation, parce qu'elle est souvent affective et parfois fusionnelle, se révèle extrêmement fragile. L'extension du domaine professionnel associée à la confusion des registres personnels et affectifs expose très fortement l'individu (mais aussi ses supérieurs dont la personnalité, les goûts deviennent aussi importants que la fonction). Comme dans les vraies familles, le moindre point de discorde peut constituer le fondement d'un processus d'éloignement très mal vécu de part et d'autre.

Ce contexte offre ainsi le cadre idoine permettant le passage d'une relation supérieurs subordonnés, assise sur une idéologisation de la fonction et sur un rapport de forces construit sur des logiques antagonistes, à une relation singulière salarié-supérieur. Le concept de harcèlement moral, dont la difficulté majeure tient à la caractérisation de l'intention, traduit ainsi le passage du pluriel au singulier et de l'objectivité à la subjectivité. 
Des configurations conflictuelles assez différenciées peuvent ainsi être analysées.

- Une première configuration est assise sur opposition entre des positions dans laquelle la personnalité de chaque salarié protégé est faiblement mobilisée et où le droit est une ressource complémentaire au soutien du collectif.

- Une deuxième configuration s'appuie sur le produit de la déception affective et a pour enjeu la stabilisation ou la reconstruction de l'identité professionnelle et sociale de l'individu qui supporte, seul ou en famille, les coûts de l'affrontement, et en particulier ses coûts psychologiques. Le droit devient alors une ressource pour un usage individuel.

Or, et cela se vérifie tout particulièrement dans cette deuxième configuration, le conflit se joue entre des instances ne disposant pas des mêmes ressources. Jugeant à partir de cette inégalité et dans bien des cas avec l'objectif avoué de réduire cette inégalité, l'intervention de l'inspecteur du travail apparaît déterminante.

Chargés de rechercher si un lien existe entre le licenciement demandé et le mandat du salarié protégé en même temps que garant de la procédure, les inspecteurs du travail cherchent, au travers de l'enquête contradictoire, à établir un équilibre dans le litige opposant l'employeur et le salarié. La motivation de leur décision constitue la traduction du passage des faits au droit.

Toutefois, la dimension subjective n'est pas absente de leur décision. Ils fondent, en premier lieu, leur décision d'une part sur les contributions des parties lors de l'enquête contradictoire, mais aussi, quand l'entreprise ou le salarié concerné leur sont connus, sur "l'impression générale" qu'ils se font des relations sociales dans l'entreprise au travers des incidents qui leur ont été auparavant signalés mais aussi de la compréhension quasi physique qu'ils peuvent avoir de l'ambiance générale.

Constitués en corps dont l'indépendance se trouve affirmée dans la convention de l'OIT $^{1}$, les inspecteurs du travail interrogés manifestent un ethos professionnel qui penche en faveur des salariés. Toutefois, leur appréciation est tempérée ou accentuée par des idéologies personnelles, c'est-à-dire, dans son sens le plus neutre, des manières particulières de voir le monde qui leur font comparer les informations tirées du terrain avec un modèle d'organisation du travail et de comportement des salariés protégés qui leur sert de référence. La qualité de leurs relations, tant avec les représentants patronaux qu'avec les représentants des salariés, influe sur leur jugement. Mais plus que tout, ils sont porteurs de certaines idées, qui orientent le point de vue qu'ils portent sur l'affaire en cours, sur ce que doivent ou devraient faire l'employeur et le représentant des salariés.

À partir de là, ils peuvent avoir une action très normative, élaborant des stratégies, notamment d'aide et de conseil auprès des salariés, afin que l'issue de la procédure corresponde à leur conception d'une solution "équitable".

\footnotetext{
${ }^{1}$ Remarquons au passage que la formulation adoptée par l'OIT "Le personnel de l'inspection sera composé de fonctionnaires publics dont le statut et les conditions de service leur assurent la stabilité dans leur emploi et les rendent indépendants de tout changement de gouvernement et de toute influence extérieure indue" reprend quasiment mot pour mot les caractéristiques de la bureaucrate comme organisation idéale telles que les décrit Weber (1921)
} 
L'intervention des autres acteurs (comité d'entreprise ou syndicat) apparaît plus marginale. Tout d'abord, l'avis du comité d'entreprise pèse peu sur la procédure, d'autant plus que pour juger des raisons de cet avis, il faut souvent une idée des événements qui ont conduit à la constitution d'une instance plus ou moins proche des positions patronales. Quant à l'organisation syndicale, elle n'est mobilisée que par les salariés protégés syndiqués et n'apporte, la plupart du temps, qu'une contribution juridique ou financière, c'est-à-dire de la logistique plus qu'un réel appui politique.

L'issue de la procédure peut prendre plusieurs configurations.

Tout d'abord, des voies de recours existent qui permettent de contester des décisions et donc de reculer d'autant l'issue de la procédure.

Toutefois, tous les salariés ne sont pas en capacité de s'engager dans des procédures de recours ou même de contester leur licenciement. L'analyse de cette population se révèle cependant difficile : certains ont été laminés par le conflit au point de ne plus vouloir en parler.

Dans les formes issues des relations les plus fusionnelles, les blessures narcissiques sont tellement profondes, l'enjeu identitaire est tellement fort que le conflit prend des formes paroxysmiques, pathologiques, au point que ce qui est recherché n'est pas la fin du conflit mais la destruction de l'adversaire. C'est alors souvent l'intervention de tiers (avocat, inspecteur du travail, médecin du travail) qui permet de sortir de cette impasse.

De plus en plus souvent, c'est par une utilisation dévoyée de la règle de droit que les protagonistes arrivent à dépasser une impasse conflictuelle. Et c'est bien parce que le conflit est dans l'impasse avec les conséquences psychologiques que cela peut avoir pour les salariés, qu'en connaissance de cause, inspecteurs du travail ou médecins participent à la construction d'une sortie de crise.

Reposant la plupart du temps sur un problème de santé qui n'impliquerait normalement pas une reconnaissance d'inaptitude, l'avis du médecin du travail permet, par une distorsion de la règle de droit, de retirer le salarié d'une situation de travail devenue insupportable. Le médecin du travail représente alors l'acteur majeur de cette forme de sortie de crise sur laquelle les inspecteurs du travail font peu de remarques dès lors que le salarié est demandeur ou consentant. Un accord peut être négocié en même temps que l'inaptitude se construit, mais, dans la plupart des cas, le salarié protégé qui est licencié pour inaptitude n'a comme avantage, bien léger, que de pouvoir prétendre aux indemnités de chômage.

A l'inverse, les salariés qui parviennent à un accord avec l'employeur font souvent payer au prix fort le fait de laisser le champ libre à l'employeur. Par un contournement de la règle de droit, le salarié protégé monnaye ce qui, en principe, ne lui appartient pas. Les inspecteurs du travail, qui sont les garants du respect de la procédure et, ce faisant, de l'intérêt collectif des salariés, ne refusent pas obligatoirement le licenciement du seul fait qu'ils ont connaissance de l'existence d'une transaction qui masque une rupture d'un commun accord. Certains le refusent si le salarié protégé occupe un mandat "d'opportunité", c'est-à-dire quand l'élection ou la désignation leur a uniquement offert une protection ou un moyen de négociation sans que le salarié n'ait jamais réellement exercé une fonction représentative. Bien que la plupart des inspecteurs du travail rencontrés aient plutôt une perception négative de la transaction, ils 
autorisent le licenciement par souci d'humanité, pour ne pas laisser le salarié dans une situation invivable.

$\mathrm{Au}$ terme de cette enquête, il ressort que la question posée par le ministère ne peut recevoir de réponse simple et que plusieurs explications complémentaires permettent de comprendre l'augmentation des demandes de licenciement adressées au ministère du travail de même que leur plus grande acceptation par les salariés protégés.

Toutefois, la plus grande importance accordée à l'individu et la généralisation de normes de comportement qui lui imposent une plus grande responsabilité dans la conduite de sa vie professionnelle et sociale apparaît comme surdéterminant pour comprendre la personnalisation des mandats comme l'individualisation des stratégies des salariés protégés. Mais, et c'est le revers de la médaille, cette individualisation explique aussi l'état de plus grande fragilité dans lequel se trouvent les individus compte tenu de leur plus grande exposition personnelle, et paraît surdéterminer l'augmentation des demandes de licenciement de cette catégorie de salariés. 


\section{BIBLIOGRAPHIE}

\section{Bibliographie sociologique}

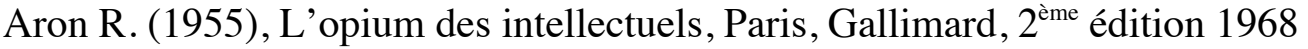

Berton F., Correia M., Maillebouis M. Lespessailles C., (2004) "La demande de formation : quelle place à l'initiative individuelle ?", l'harmattan, à paraître.

Boltanski L. et Thévenot L. (1991), "De la justification; les économies de la grandeur", Paris, Gallimard.

Boudon R. (1977), "Effets pervers et ordre social", PUF.

Boudon R., Bourricaud F. (sous la direction de) (1986), "Dictionnaire Critique de la Sociologie", PUF, 1986 (2e édition).

Bourdieu P. (1980), "Questions de sociologie", Paris, Ed de Minuit.

Bourricaud F. (2002), "Conflits sociaux", in Encyclopédie Universalis.

Charlot B., Bautier E., Kohn R., Rochex J.-Y. (1992), "Rapport au savoir et rapport à l'école dans les zones d'éducation prioritaires", Rapport pour le F.A.S. et la D.P.M., Février 1992.

Chenu A. (1990), "L'archipel des employés", Ed. INSEE Etudes

Corcuff P. (2002), La société de verre, Armand Collin.

Correia M. (1994), "Les mobiles des trajectoires individuelles: du jugement sur la justice à la recherche d'une nouvelle légitimité sociale", thèse de doctorat de sociologie.

Correia Mario, Pottier François (2001), "Usages et réussite des parcours de formations suivies à l'initiative des individus : le cas du CNAM", journées du Lasmas-IdL, Séance du lundi 2 avril 2001.

Correia M., Lenel P., Leroux N. (1999), "Entre désinvestissement, promotion et mobilité diagonale : le cas des "surdiplômés" du Ministère de l'équipement, du logement, des transports et du tourisme", doc. interne, Ministère de l'équipement, du logement, des transports et du tourisme, 158 pages.

Correia M. (2003), "L'individualisation de la relation de travail dans la "fonction publique", in Chauchard J.-P., Hardy-Dubernet A.-C. (dir.), " La subordination dans le travail", Travail et Emploi, pp. 177-188

Demazières D., Dubar C. (1997), "Analyser les entretiens biographiques", Nathan.

De Singly F. (2003), "Les uns avec les autres", Armand Collin

Dubois M. (1993), Documents de travail, n 87, CEREQ, Septembre 1993.

D'Iribarne P. (1989), "La logique de l'honneur", Ed Seuil, 1989

Dugué E. (1995), "Les étudiants d'économie et gestion : trajectoires et usages professionnels du CNAM au moment du diplôme", Juillet, doc. interne CNAM.

Durkheim E. (1893), "De la division du travail social", PUF (édition, 1960)

Ehrenberg A. (1998), "La fatigue d'être soi. Dépression et société", Paris, Odile Jacob

Ehrenberg A. (1995) "L’individu incertain", Calmann-Lévy coll. essai société

Elias N. (1981), "Qu'est ce que la sociologie", Éditions de l'aube.

Enriquez E (1983)., "De la horde à l'état", Paris, Gallimard.

Enriquez E. (1991), "Les figures du maître", Paris, Arcantère, 1991.

Goffman E., (1973) "La mise en scène de la vie quotidienne", Tome 1, "La présentation de soi", Paris, Les Éditions de Minuit. 
Goffman E. (1973), "La mise en scène de la vie quotidienne", Tome 2: "La vie en public", Paris, Les Éditions de Minuit.

Hirschman Albert (1986), "Défection ("exit") et prise de parole ("voice): l'état du débat", in "Vers une économie politique élargie", Ed de Minuit.

Joule R. V., Beauvois J. L. (1987), "Traité de manipulation à l'usage des honnêtes gens", Grenoble, Presses Universitaires de Grenoble, 1987.

Lahire B. (1998), L'homme pluriel. Les ressorts de l'action, Nathan.

Lorenz E. (1996), "Confiance, contrats et coopération économique", Sociologie du travail $\mathrm{n}^{\circ} \mathrm{XXXVIII,} \mathrm{4/96.)}$

Maggi-Germain N., Correia Mario (2001), "L'évolution de la formation professionnelle continue : regards juridique et sociologique", droit Social $n^{\circ}$ 9/10 septembre-octobre 2001.

Marchand O., Thélot C. (1997), "Le travail en France (1800-2000)", Nathan.

Memmi A. (1979), "La dépendance", Paris, Gallimard.

Mintzberg H. (1982), "Structure et dynamique des organisations", Ed. Des Organisations.

Olson O., (1964) "Logique de l'action collective" (1964. trad. française PUF 1978)

Piotet F., Correia M., Lattes C., Vincent J. (1993), "Le développement de la syndicalisation à la CFDT", IRES-CESTE, octobre.

Piotet F., Correia M., Lattès C., Vincent J. (1994), "Le développement de la syndicalisation à la CFDT", Juin 1994, Publication CESTE-IRES

Pottier F. (1992), "De l'enseignement supérieur à l'emploi", Documents O.E.C.D..

Reynaud J. D. (1989), "Les règles du jeu. L'action collective et la régulation sociale", Paris, Armand Collin.

Saglio J. (1991), "Echange social et identité collective dans les systèmes Industriels", Sociologie du travail, 4-1991

Sainsaulieu R. (éd.) (1990), "L'entreprise, une affaire de société", Paris, Presses de la Fondation Nationale des Sciences Politiques.

Serres M. (1991), "Le tiers instruit", Gallimard.

Simmel G (2003)., "Le conflit", Circé.

Tixier (1992), "La fin du syndicalisme", P.U.F.

Touraine A. (1965), "Sociologie de l'action", Paris, Seuil.

Weber M. (1959), "Le savant et le politique", Paris, Ed C. Bourgois.

Weber M. (1922), Economie et société, t. 1, Les catégories de la sociologie, Paris: Plon (Pocket), 1995 (1ère éd. 1922)

\section{Bibliographie juridique}

\section{Ouvrages et thèses}

Kerbourc'h (J-Y), Le statut protecteur des représentants du personnel, LITEC, 2003, 343 p.

Le Crom (J.-P.), L'introuvable démocratie salariale. Le droit de la représentation du personnel dans l'entreprise (1890-2002), Paris, Syllepse, coll. Le Présent Avenir, 2003, $194 \mathrm{p}$. 
Melot (R), Entre contrat et procès : enquête sur les transactions entre employeur et salarié, Thèse pour le doctorat en sociologie, Déc. 2003, Ecole normale supérieure de Cachan, $552 \mathrm{p}$.

Mine (M), Rose $(\mathrm{H})$ et Struillou (Y), Le droit du licenciement des salariés protégés, Paris, Economica, 1996, 663 p.

Puigelier (C), La rupture du contrat de travail du salarié protégé, Paris, Economica, coll. droit social pratique, $1998,121 \mathrm{p}$.

Supiot (A), Les juridictions du travail, tome 9 du Traité de droit du travail (dir. G.-H. Camerlynck), Dalloz, 1987, 768 p.

Verdier (J.-M.), Syndicats et droit syndical, tome 5 du Traité de droit du travail (dir. G.-H. Camerlynck), Dalloz,, Paris, 1966, mise à jour 1976.

\section{Articles}

Thévenot (P.-H.), «L'obligation de reclassement préalable au licenciement pour motif économique : nouvelles dispositions», droit social 2002, p. 274 à 278.

Béraud (J.-M.), "L'influence de la suspension du contrat de travail sur les relations institutionnelles dans l'entreprise", Dr. Soc. 1980, p. 151 à 164.

Borenfreund (G), droit syndical dans l'entreprise, Répertoire Dalloz droit du travail (syndicats professionnels).

Cahiers sociaux du barreau de paris, «Différences de traitement, en mêmes circonstances, entre salariés protégés et salariés non protégés», 1997, nº 91, p. 181 à 182

Charbonneau, "Rupture négociée du contrat du représentant du personnel", Cahiers sociaux du Barreau de Paris n 144, 2002, D 18, p. 439 à 441.

Chelle (D) et Prétot (X), "Licenciement du salarié protégé : nouveaux développements de la jurisprudence administrative", RJS 1992, p. 457.

Cohen (M), «Les droits des salariés protégés en cas de changement d'employeur», droit social 1997, p. 263 à 269.

Corrignan-Carsin (D), note sous Cass. Soc. 28 mars 2000, JCP 2000, II, 10347.

Coudray $(\mathrm{O})$, «Le juge administratif et le licenciement des salariés protégés, Action juridique CFDT nº 149, 2001, p. 7 à 18.

Duquesne (F), "Représentants du personnel. Contrat de travail et exercice du mandat", RJS 7/02, p. 603 à 608.

Duquesne (F), "Le régime de la mise à pied du salarié protégé gréviste", Dr. Soc. 2003, p. 177 à 182.

Équipe rédactionnelle de la revue fiduciaire, «Le licenciement des salariés protégés, Revue fiduciaire $\mathrm{n}^{\circ} 879,2000$.

Frouin (J.-Y), "L’indemnisation des salariés protégés licenciés sans autorisation", RJS 11/01, p. 842 à 848 .

Furjot (D), "Où sont les délégués syndicaux ?", Premières synthèses, Mes-Dares 2000, n 412. 
Gherari $(\mathrm{H})$, «Le juge administratif et la procédure de licenciement des salariés protégés dans l'entreprise», Les Petites affiches n 7, 1997, p. 8 à 13.

Hoyez $(\mathrm{K})$, «L'inspecteur du travail et le licenciement d'un représentant du personnel malade ou inapte», Travail et protection sociale $\mathrm{n}^{\circ} 6,1999$, chron. 10, p. 4 à 5 .

Javillier (J.-Cl.), "Syndicat et représentation élue", Dr. Soc. 1984, p. 31.

Latournerie (R), "L'interprétation des textes assurant la protection des représentants du personnel dans l'entreprise", D. 1975, Chron., p. 104.

Maggi-Germain $(\mathrm{N})$, «Statut et contrat: deux modes de construction de la relation de travail », La Revue de l'Ires, numéro spécial ( $\left.\mathrm{n}^{\circ} 45\right)$ Les relations professionnelles dans le secteur public, 2004/2, p. 103 à 117.

Maggi-Germain (N), «Le stress au travail», RJS 3/2003, p. 191 à 200.

Maggi-Germain $(\mathrm{N})$, «Harcèlement moral et conditions de travail», Bulletin social Francis Lefebvre ${ }^{\circ} 8 / 9,2002$, p. 470 à 472.

Maggi-Germain (N), «Travail et santé : le point de vue d'une juriste», Dr. soc. mai 2002 p. 485 à 493.

Maggi-Germain N., Correia Mario (2001), "L'évolution de la formation professionnelle continue : regards juridique et sociologique", Dr. Social $n^{\circ}$ 9/10 septembre-octobre 2001.

Jacques (C), "Travail et santé : le point de vue du médecin", Dr. Soc. mai 2002, p. 479 à 484.

Merlier R. et Bauduret (M.-Ch.), "Les licenciements des représentants du personnel en 1996", Premières informations et premières synthèses n 45.1, 1997, p. 1 à 6.

Bauduret (M.-Ch.) et Merlier R., "Les licenciements des représentants du personnel en 1997", Premières informations et premières synthèses $\mathrm{n}^{\circ} 171,1999$, p. 1 à 6.

Melot R., "Les règlements contractuels des litiges entre salarié et employeur : de la gestion des litiges à la gestion des compétences ?", in Contraintes, normes et compétences au travail. Les régimes de mobilisation, Publications du Centre Pierre Naville, 2003, tome 3, p. 109 à 119, p. 119.

Melot R., Entre contrat et procès : enquête sur les transactions entre employeur et salarié, Thèse de sociologie soutenue à l'Ecole normale supérieure de Cachan, nov. 2003, 552 p.

Merlier R., "Les licenciements des représentants du personnel en 1998", Premières informations et premières synthèses $\mathrm{n}^{\circ} 19.1,2000$, p. 1 à 7.

Merlier R., "Les licenciements des représentants du personnel en 1999", Premières informations et premières synthèses $\mathrm{n}^{\circ} 47.1$, nov. 2001, p. 1 à 7.

Merlier R., "Les licenciements des représentants du personnel en 2000", Premières informations et premières synthèses $\mathrm{n}^{\circ} 48.2,2002$, p. 1 à 7.

Merlier R., De Oliveira V., Zilberman S., "Les licenciements de salariés protégés de 2001 à 2003 : le motif économique prédominant, toujours et encore", Premières informations et premières synthèses $\mathrm{n}^{\circ} 38.3,2005$.

Ministère du travail et des affaires sociales, Dares, "La délégation unique du personnel, un développement significatif en 1994", Premières informations n 96-9.2, 23 février 1996.

Pélissier (J), "La fonction syndicale dans l'entreprise", Dr. Soc. 1984, p. 41 
Reymann (I), «Conséquences civiles et pénales du défaut d'autorisation de l'inspecteur du travail dans le cadre du licenciement d'un salarié protégé», Les Petites affiches $\mathrm{n}^{\circ} 172$, 1999, p. 4 à 5.

Semaine sociale Lamy, «Salariés protégés», $\mathrm{n}^{\circ} 761,1995$, p. 6.

Sinay (H), "Un tournant du droit du travail : les arrêts Perrier", Dalloz 1974, Chron., p. 235

Supiot (A), "Revisiter les droits d'action collective", Dr. Soc. 2001, p. 687 à 704.

Supiot (A), "La fonction anthropologique du droit", Entretien, Esprit, février 2001, p. 151 à 173.

Touffait, conclusions sous Cass. Chambre mixte 21 juin 1974, Perrier, Dalloz 1974, p. 593 à 604.

Verdier (J.-M.), "Du contrat au statut et du droit individuel aux libertés publiques", JCP, 1971, I, 2422.

Verdier (J.-M.), «La protection des représentants des salariés dans l'entreprise : une logique de statut »,Dr.Soc. 2001, p. 634 à 638.

Verdier (J.-M.), "Rétractation de l'autorisation administrative du licenciement d'un salarié protégé : nouveaux progrès de la logique du statut", Dr. Soc. 2002, p. 724 à 727.

Verdier (J.-M.), «Les représentants des salariés: «protégés» ou exposés ? Liberté fondamentale et logique du statut», in Analyse juridique et valeurs en droit social, Mélanges en l'honneur de Jean Pélissier, Dalloz, 2004, 631 p., p. 571 à 590.

\section{Rapports et études}

Thévenot (J-Cl), psychanalyste, CLANCY (S), psychologue clinicienne, MICHEL (A), sociologue, PASCUAL (M), médecin du travail, Salariés en détresse: quelles réponses? L'expérience d'une consultation pluridisciplinaire, Consultation "Souffrance et travail", fnath 11 rue du Chemin vert, 75011 Paris

Barrat O., DANIEL C. (2001), "Dynamique de la négociation d'entreprise en 1999 et 2000 : l'effet des lois de réduction du temps de travail", Dares, Premières synthèses, $\mathrm{n}^{\circ}$ 19.2, mai.

J. Bessière, L'inspection du travail, Rapport au Ministre délégué aux relations du travail, janvier 2005, $128 \mathrm{p}$.

Coutrot T. (2001), "Où sont les CHSCT ?", Dares, Premières synthèses n 16.2, avril.

Furjot D. (2000), "Où sont les délégués syndicaux", Dares, Premières synthèses n 41.2 , octobre.

Furjot (D), De la participation au conflit. Point de vue et pratiques des institutions représentatives du personnel en matières de relations professionnelles, Enquête "réponse" 1998 questionnaire "représentants du personnel", DARES, Document d'études n 64, décembre 2002, 82 p.

Leclair $(\mathrm{M})$ et Petit $(\mathrm{P})$, Présence syndicale dans les firmes : quel impact sur les inégalités salariales entre les hommes et les femmes ?, INSEE, doc. de travail G 2003/10, déc. 2003, 44 p. 
Ministère des affaires sociales, du travail et de l'emploi (2002), La négociation collective en 2001, Ministère des affaires sociales, du travail et de l'emploi et Editions législatives, Tome 1.

Ministère des affaires sociales, du travail et de la solidarité (DAGEMO), L'inspection du travail en France en 2002. Les chiffres clés, Rapport au B.I.T., 302 p., p. 72.

Pignoni (M.-T.) et Zouary (P), «Les nouveaux usages du licenciement pour motif personnel», Dares, Premières informations premières synthèses $\mathrm{n}^{\circ} 28.2$, juillet 2003, 9 p.

Ruelland N. (2001), "Les institutions représentatives du personnel en 1999", Dares, Première information, $\mathrm{n}^{\circ} 48.1$, nov.

\section{Documents administratifs}

Circulaire DRT n ${ }^{\circ}$ 96-11 du 3 décembre 1996, Ministère du travail et des affaires sociales, Dr. Ouvrier mai 1997, p. 201 à 207.

Circulaire DRT n ${ }^{\circ} 3$ du $1^{\text {er }}$ mars 2000, Ministère de l'emploi et de la solidarité, Dr. Ouvrier oct. 2000, p. 442 à 451

Circulaire DRT n ${ }^{\circ}$ 2002/17 du 16 septembre 2002, Ministère de l'emploi et de la solidarité, Semaine sociale Lamy. 14 oct. 2002, p. 2 à 3. 